\title{
Sequence-Selective Decapeptide Synthesis by the Parallel Operation of Two Artificial Molecular Machines
}

Javier Echavarren, Malcolm A. Y. Gall, Adrian Haertsch, David A. Leigh, ${ }^{*}$ Justin T. J. Spence, Daniel J. Tetlow and Chong Tian

School of Chemistry, University of Manchester, Oxford Road, Manchester M13 9PL, UK.

*E-mail: david.leigh@manchester.ac.uk

\section{Supporting Information}

Table of Contents

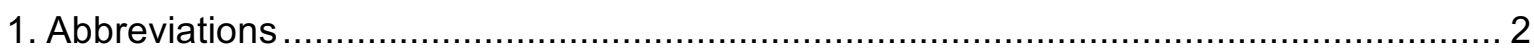

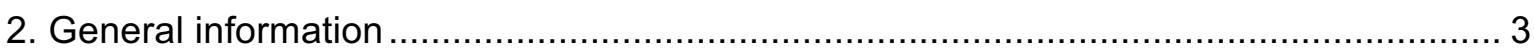

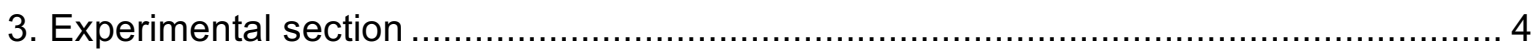

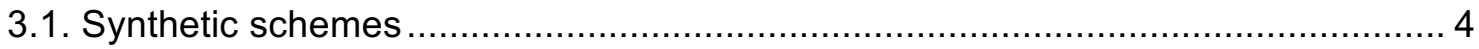

3.2. Synthetic procedures and characterization details ........................................... 7

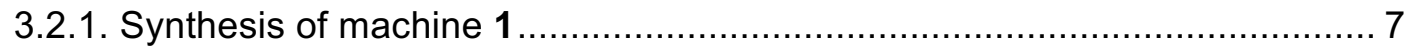

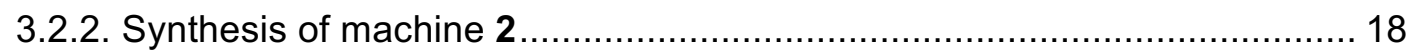

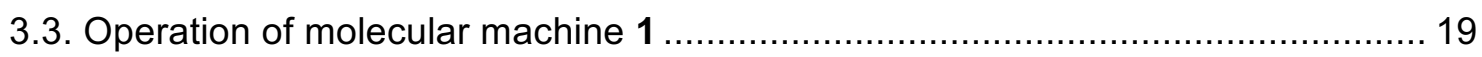

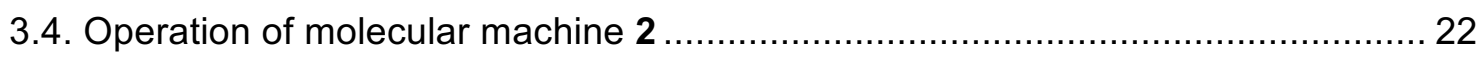

3.5. Operation of molecular machines 1 and 2 in one pot .................................... 25

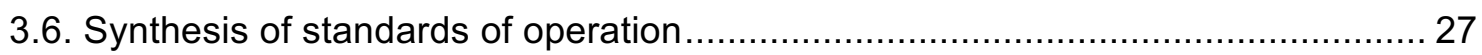

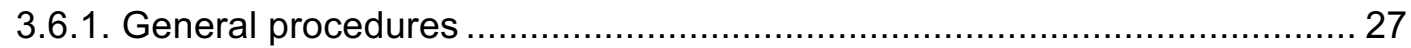

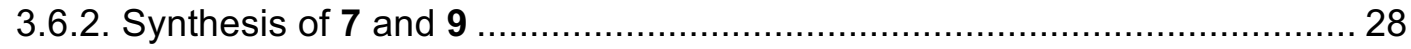

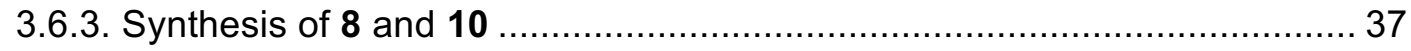

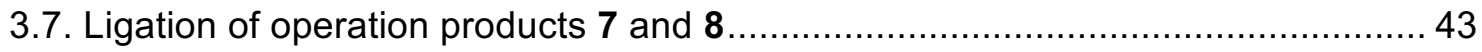

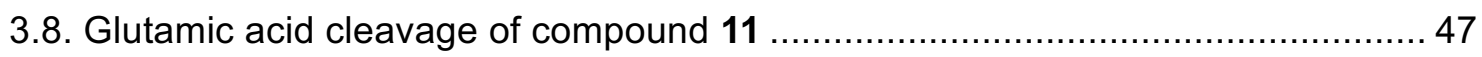

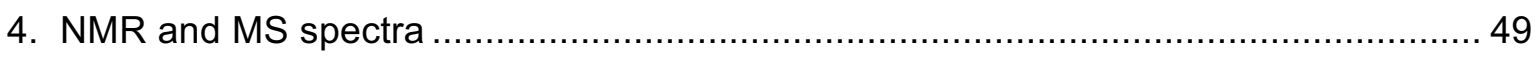

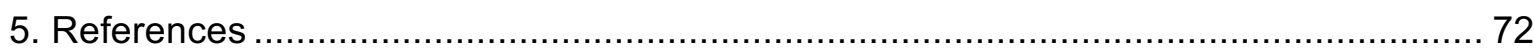




\section{Abbreviations}

Ala alanine; Boc tert-butoxycarbonyl carbamate; CuACC Copper-catalyzed azide-alkyne cycloaddition; DIPEA diisopropylethylamine; DMAP 4-N,N-dimethylaminopyridine; DMF N,Ndimethylformamide; DMSO dimethylsulfoxide; EDTA ethylenediaminetetraacetic acid; $\mathrm{EDCl} \cdot \mathrm{HCl}$ 1-ethyl-3-(3-dimethylaminopropyl) carbodiimide hydrochloride; Fmoc Fluorenylmethoxycarbonyl; Glu glutamic acid; HFIP 1,1,1,3,3,3-Hexafluoro-2-propanol; $h$ hours; Leu Leucine; MPPA 4-Mercaptophenylacetic acid; MeCN acetonitrile; min minutes; Phe phenylalanine; PTLC preparatory thin layer chromatography; quant. quantitative yield, rt room temperature; TASF tris(dimethylamino)sulfonium difluorotrimethylsilicate; TBAF tetranbutylammonium fluoride; TBDPS-Cl tert-butyl(chloro)diphenylsilane; TBTA tris[(1-benzyl-1H1,2,3-triazol-4-yl)methyl]amine; THF tetrahydrofuran; Thi Thioproline; TIPS triisopropylsilyl; TLC thin layer chromatography; TMS trimethylsilyl; Trt trityl; Tyr tyrosine. 


\section{General information}

${ }^{1} \mathrm{H}$ NMR spectra were recorded on a Bruker Avance III instrument with an Oxford AS600 magnet equipped with a cryoprobe [5mm CPDCH 13C-1H/D] $(600 \mathrm{MHz})$ at $298 \mathrm{~K}$. Chemical shifts are reported in parts per million (ppm) from high to low frequency using the residual solvent peak as the internal reference DMSO- $d_{6}=2.50 \mathrm{ppm}, \mathrm{CDCl}_{3}=7.26 \mathrm{ppm}$, Acetonitrile$d_{3}=1.94 \mathrm{ppm}$ and Acetone $-d_{6}=2.05 \mathrm{ppm}$ ). All ${ }^{1} \mathrm{H}$ resonances are reported to the nearest 0.01 ppm. The multiplicity of ${ }^{1} \mathrm{H}$ signals are indicated as: $\mathrm{s}=$ singlet; $\mathrm{d}=$ doublet; $\mathrm{t}=$ triplet; $\mathrm{q}=$ quadruplet; $\mathrm{m}=$ multiplet. Coupling constants $(J)$ are quoted in $\mathrm{Hz}$ and reported to the nearest $0.1 \mathrm{~Hz} .{ }^{13} \mathrm{C}$ NMR spectra were recorded on the same spectrometer at $298 \mathrm{~K}$ with the central resonance of the solvent peak as the internal reference DMSO- $d_{6}=39.52 \mathrm{ppm}, \mathrm{CDCl}_{3}=77.16$ ppm, Acetonitrile- $d_{3}=118.26 \mathrm{ppm}$ and Acetone- $d_{6}=29.84 \mathrm{ppm}$ ). All ${ }^{13} \mathrm{C}$ resonances are reported to the nearest $0.01 \mathrm{ppm}$ to aid in the differentiation of close but resolved signals. Flash column chromatography was carried out using Silica $60 \AA$ (particle size $40-63 \mu \mathrm{m}$, Sigma Aldrich, UK) as the stationary phase. Preparative TLC was performed using PLC $20 \times$ $20 \mathrm{~cm}, 60 \mathrm{~F}_{254}$ preparatory plates (Merck). Analytical TLC was performed on precoated silica gel plates $\left(0.25 \mathrm{~mm}\right.$ thick, $60 \mathrm{~F}_{254}$, Merck, Germany) and visualized using both short and long wave ultraviolet light in combination with standard laboratory stains (ceric ammonium molybdate). Low resolution ESI mass spectrometry was performed with a Thermo Scientific LCQ Fleet Ion Trap Mass Spectrometer. High-resolution mass spectrometry was carried out by the Mass Spectrometry Service, Department of Chemistry, The University of Manchester. Compounds S9 (stopper), ${ }^{1} \mathbf{S} 23$ (aldehyde macrocycle) ${ }^{2}, \mathbf{S 1}$ (unloaded internal barrier) ${ }^{2}, \mathbf{S 3}$ (unloaded terminal barrier) $)^{2}, \mathbf{S 5}$ (Leu-barrier) $)^{2}, 6$ (2-barrier tailpiece) $)^{2}, 4$ (1-barrier rotaxane) $)^{3}$, $\mathbf{S 1 8}$ (peptide) ${ }^{4}$, S6 (amide macrocycle) ${ }^{5}$ were prepared according to previously reported procedures. 


\section{Experimental section}

\subsection{Synthetic schemes}<smiles>C#Cc1ccc(C(=O)NC(Cc2ccc(O)cc2)C(=O)Nc2ccc(C(C)(C)c3ccc(N)cc3)cc2)cc1</smiles>
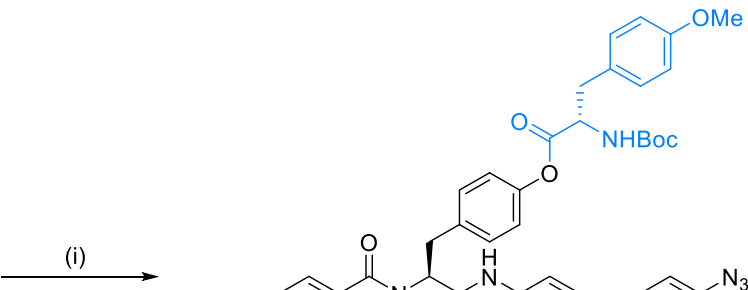

s1

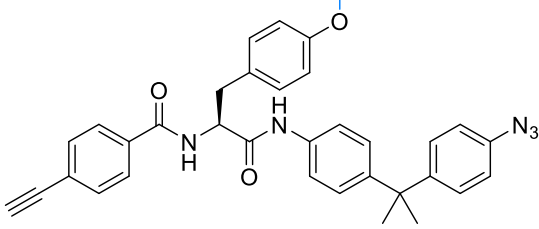

s2

Scheme S1. Synthesis of barrier S2. Reagents and conditions: (i) Boc-Tyr(Me)-OH, EDCl-HCl, DMAP, $\mathrm{CHCl}_{3}: \mathrm{THF}, \mathrm{rt}, 18 \mathrm{~h}, 93 \%$.<smiles>C#Cc1ccc(C(=O)NC(Cc2ccc(O)cc2)C(=O)Nc2ccc(C(C)(C)c3ccc(NC(=N)Nc4ccccc4)cc3)cc2)cc1</smiles>

S3

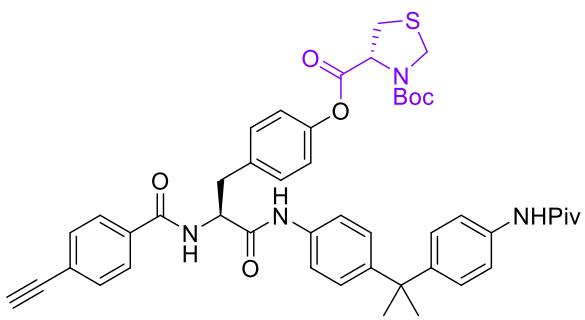

S4

Scheme S2. Synthesis of barrier S4. Reagents and conditions: (i) Boc-Thi-OH, EDCI-HCl, DMAP, $\mathrm{CHCl}_{3}: \mathrm{THF}, \mathrm{rt}, 20 \mathrm{~h}, 67 \%$.
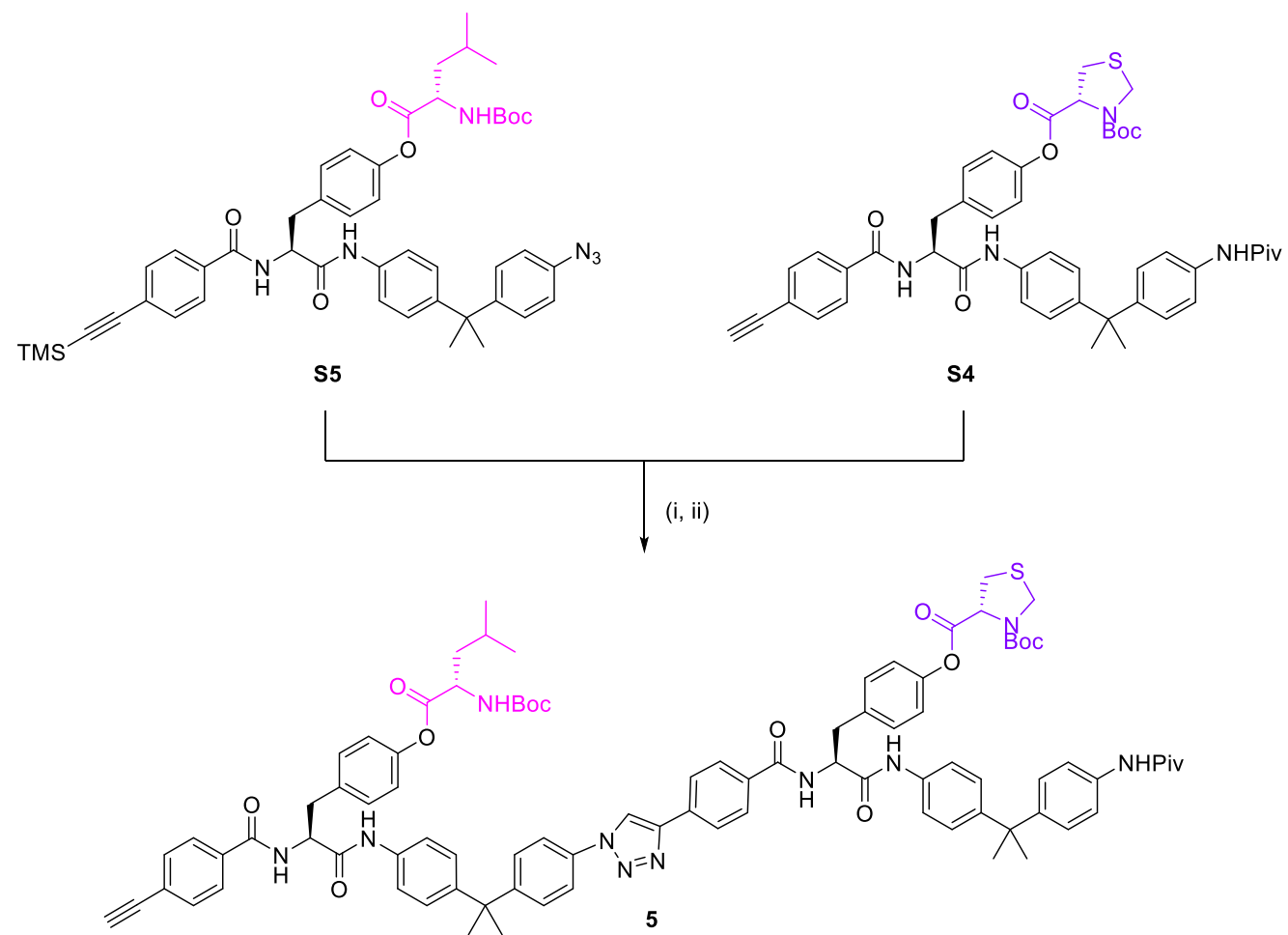

Scheme S3. Synthesis of 2-Barrier Extension Piece 5. Reagents and conditions: (i) $\mathrm{Cu}(\mathrm{MeCN})_{4} \mathrm{PF}_{6}$, Tentagel $^{\mathrm{TM}}$ TBTA resin, $\mathrm{CH}_{2} \mathrm{Cl}_{2}, t-\mathrm{BuOH}$, rt, 20 h. (ii) TASF, DMF, rt, 1 min, $66 \%$ over 2 steps. 


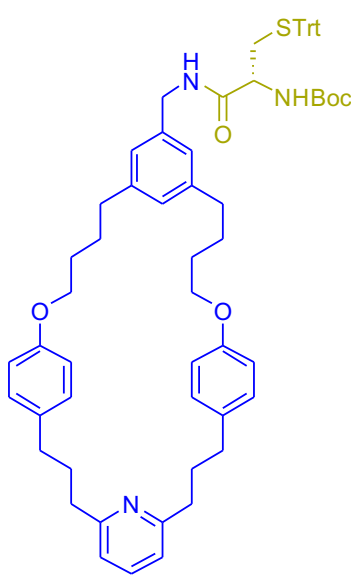

s6

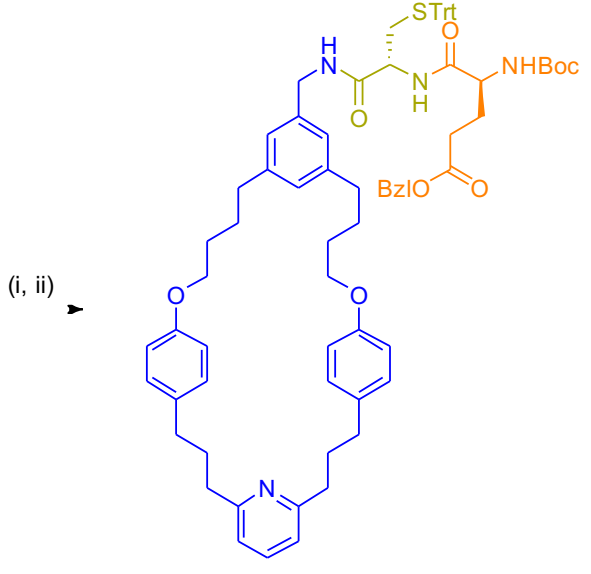

S7

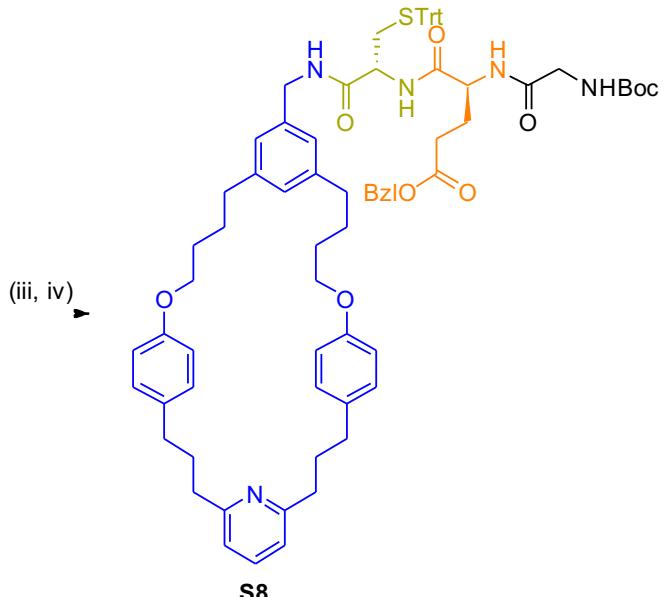

S8

Scheme S4. Synthesis of macrocycle S8. Reagents and conditions: (i) $\mathrm{CF}_{3} \mathrm{CO}_{2} \mathrm{H}, \mathrm{Ph}_{3} \mathrm{COH}, \mathrm{CH}_{2} \mathrm{Cl}_{2}$, rt, 90 min, quant. (ii) Boc-Glu(OBzl)-OH, EDCl. $\mathrm{HCl}, \mathrm{HOBt} \cdot \mathrm{H}_{2} \mathrm{O}, \mathrm{CH}_{2} \mathrm{Cl}_{2}$ rt, $18 \mathrm{~h}, 68 \%$. (iii) $\mathrm{CF}_{3} \mathrm{CO}_{2} \mathrm{H}$, $\mathrm{Ph}_{3} \mathrm{COH}, \mathrm{CH}_{2} \mathrm{Cl}_{2}$, rt, 90 min, quant. (iv) Boc-Gly-OH, $\mathrm{EDCl} \cdot \mathrm{HCl}, \mathrm{HOBt} \cdot \mathrm{H}_{2} \mathrm{O}, \mathrm{THF}, \mathrm{CHCl}_{3}, \mathrm{rt}, 18 \mathrm{~h}, 92 \%$.

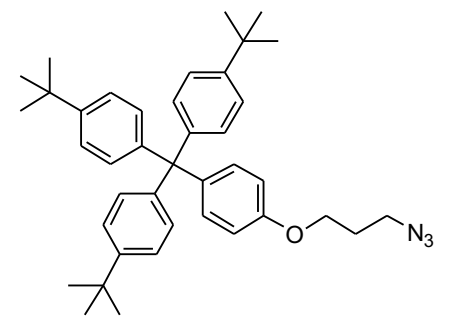

s9

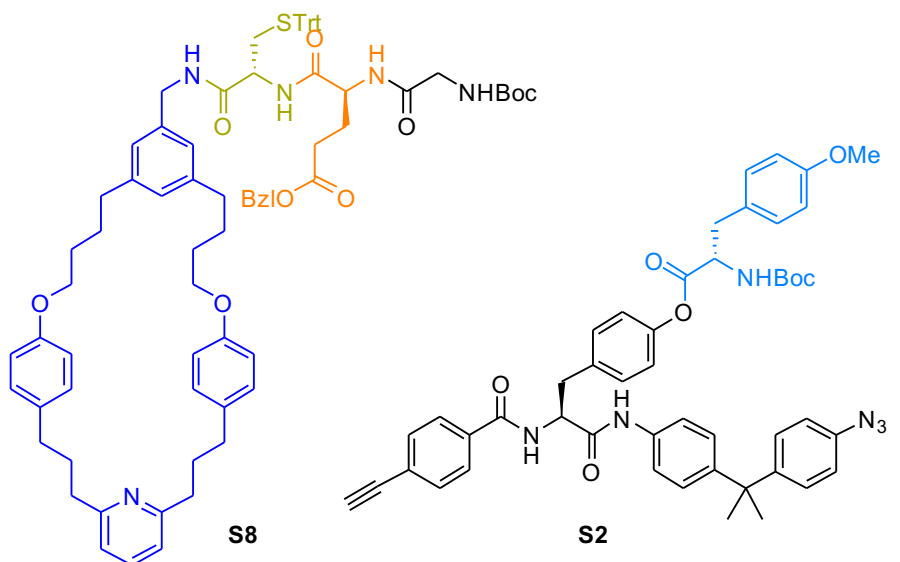

(i)

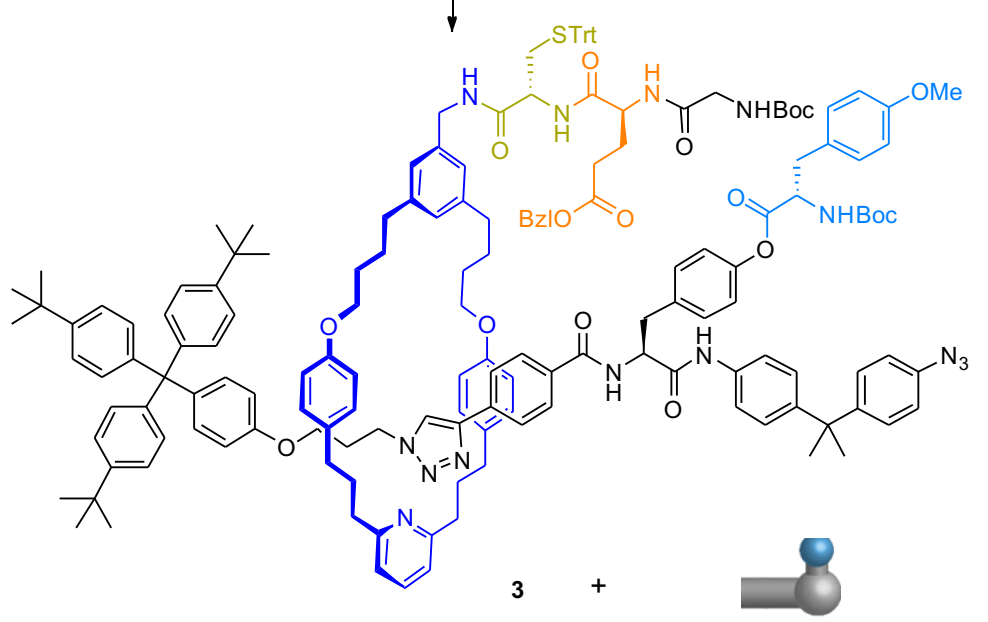

Free thread $\mathbf{S 1 0}$

Scheme S5. Synthesis of 1-Barrier Rotaxane 3 and free thread S10. Reagents and conditions: (i) $\mathrm{Cu}(\mathrm{MeCN})_{4} \mathrm{PF}_{6}, \mathrm{CH}_{2} \mathrm{Cl}_{2}, t-\mathrm{BuOH}$, rt, $24 \mathrm{~h}, 19 \%$ (rotaxane 3), 29\% (free thread S10). 

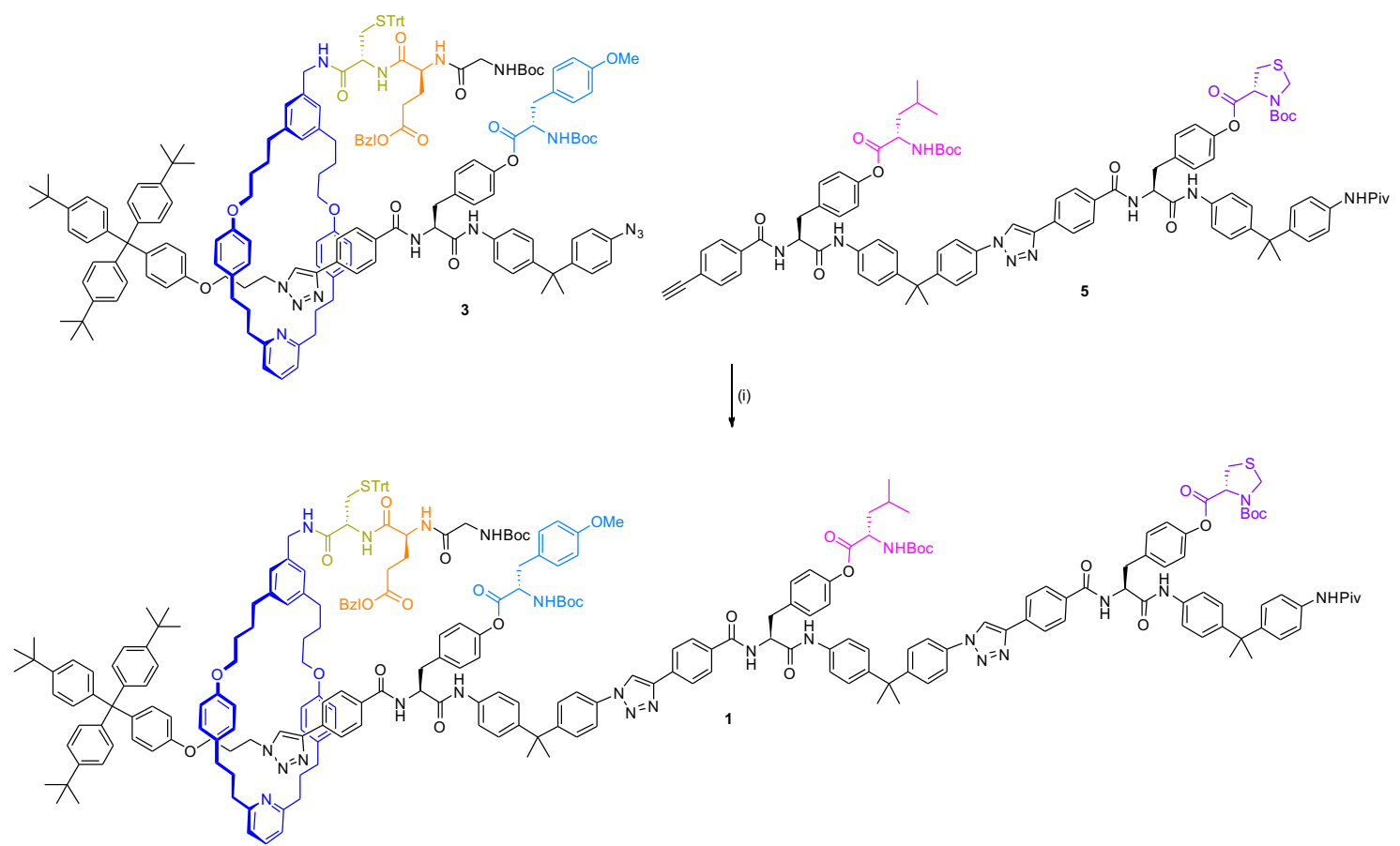

Scheme S6. Synthesis of amide machine 1. Reagents and conditions: (i) $\mathrm{Cu}(\mathrm{MeCN})_{4} \mathrm{PF}_{6}$, Tentagel $^{\mathrm{TM}}$ TBTA resin, $\mathrm{CH}_{2} \mathrm{Cl}_{2}, t$-BuOH, rt, $48 \mathrm{~h}, 63 \%$.
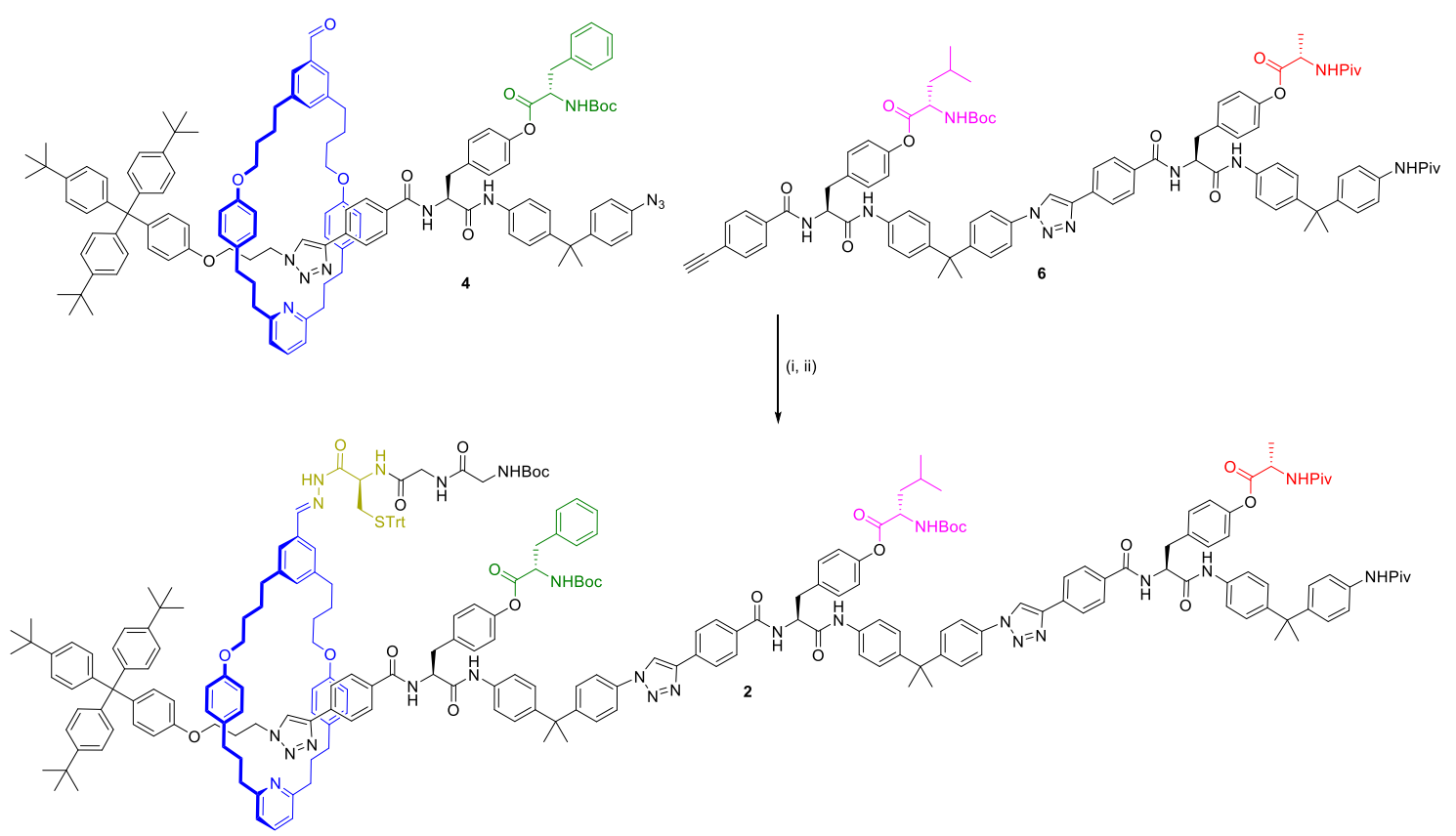

Scheme S7. Synthesis of hydrazone machine 2. Reagents and conditions: (i) $\mathrm{Cu}(\mathrm{MeCN})_{4} \mathrm{PF}_{6}$, Tentagel ${ }^{\mathrm{TM}}$ TBTA resin, $\mathrm{CH}_{2} \mathrm{Cl}_{2}, t-\mathrm{BuOH}$, rt, $48 \mathrm{~h}, 86 \%$. (ii) Boc-Gly-Gly-Cys(Trt)- $\mathrm{NHNH}_{2}$, aniline, $\mathrm{CH}_{2} \mathrm{Cl}_{2}$, rt, $48 \mathrm{~h}, 50 \%$. 


\subsection{Synthetic procedures and characterization details}

\subsubsection{Synthesis of machine 1}

S2

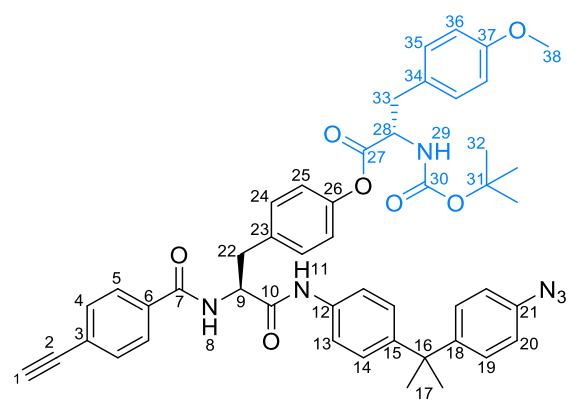

To a solution of barrier $\mathbf{S} 1$ (176 $\mathrm{mg}, 0.32 \mathrm{mmol}, 1.0$ equiv.) in $\mathrm{CHCl}_{3}: \operatorname{THF}(1: 3,6.5 \mathrm{~mL})$ was added Boc-Tyr(Me)-OH (115 mg, 0.391 .2 equiv.), DMAP (4 mg, $0.03 \mathrm{mmol}, 0.1$ equiv.) and $\mathrm{EDCl} \cdot \mathrm{HCl}(68 \mathrm{mg}, 0.36 \mathrm{mmol}, 1.2$ equiv.). The reaction mixture was stirred for $18 \mathrm{~h}$ at room temperature. The solvent was removed under reduced pressure. Purification by flash column chromatography $\left(\mathrm{SiO}_{2}, \mathrm{EtOAc} / \mathrm{CH}_{2} \mathrm{Cl}_{2} 1: 19\right)$ yielded $\mathbf{S 2}$ as a colorless solid (249 $\mathrm{mg}, 0.30$ $\mathrm{mmol}, 93 \%)$.

${ }^{1} \mathrm{H}$ NMR $\left(600 \mathrm{MHz}\right.$, Acetone- $\left.d_{6}\right) \delta 9.41\left(\mathrm{~s}, 1 \mathrm{H}, \mathrm{H}_{11}\right), 8.04\left(\mathrm{~d}, J=7.9 \mathrm{~Hz}, 1 \mathrm{H}, \mathrm{H}_{8}\right), 7.90-7.84$ $\left(\mathrm{m}, 2 \mathrm{H}, \mathrm{H}_{5}\right), 7.57-7.51\left(\mathrm{~m}, 4 \mathrm{H}, \mathrm{H}_{4}+\mathrm{H}_{13}\right), 7.41-7.35\left(\mathrm{~m}, 2 \mathrm{H}, \mathrm{H}_{24}\right), 7.29-7.27\left(\mathrm{~m}, 2 \mathrm{H}, \mathrm{H}_{20}\right)$, $7.26\left(\mathrm{~d}, J=8.6 \mathrm{~Hz}, 2 \mathrm{H}, \mathrm{H}_{35}\right), 7.19-7.14\left(\mathrm{~m}, 2 \mathrm{H}, \mathrm{H}_{14}\right), 7.01-6.98\left(\mathrm{~m}, 2 \mathrm{H}, \mathrm{H}_{19}\right), 6.98-6.95$ $\left(\mathrm{m}, 2 \mathrm{H}, \mathrm{H}_{25}\right), 6.90-6.86\left(\mathrm{~m}, 2 \mathrm{H}, \mathrm{H}_{36}\right) 6.36\left(\mathrm{~d}, J=7.9 \mathrm{~Hz}, 1 \mathrm{H}, \mathrm{H}_{29}\right), 4.99(\mathrm{td}, J=8.2,5.7 \mathrm{~Hz}$, $\left.1 \mathrm{H}, \mathrm{H}_{9}\right), 4.51\left(\mathrm{td}, J=8.2,6.0 \mathrm{~Hz}, 1 \mathrm{H}, \mathrm{H}_{28}\right), 3.83\left(\mathrm{~s}, 1 \mathrm{H}, \mathrm{H}_{1}\right), 3.77\left(\mathrm{~s}, 3 \mathrm{H} \mathrm{H}_{38}\right), 3.35$ (dd, $J=14.0$, $5.8 \mathrm{~Hz}, 1 \mathrm{H}, \mathrm{H}_{22^{\prime}}$ ), $3.21-3.14\left(\mathrm{~m}, 2 \mathrm{H}, \mathrm{H}_{22}+\mathrm{H}_{33}\right), 3.09$ (dd, J = 14.0, 8.6 Hz, $\left.1 \mathrm{H}, \mathrm{H}_{33^{\prime}}\right), 1.65(\mathrm{~s}$, $\left.6 \mathrm{H}, \mathrm{H}_{17}\right), 1.37\left(\mathrm{~s}, 9 \mathrm{H}, \mathrm{H}_{32}\right) \mathrm{ppm} ;{ }^{13} \mathrm{C}$ NMR $\left(151 \mathrm{MHz}\right.$, Acetone- $\left.d_{6}\right) \delta 171.66,170.29,166.80$, $159.65,156.33,150.53,148.72,146.56,138.10,137.47,136.31,135.25,132.69,131.24$, $131.14,129.76,129.15,128.44,127.77,126.16,122.25,120.20,120.11,119.41,114.65$, $83.46,81.39,79.52,60.53,56.76,56.68,55.46,42.89,37.84,37.23,30.98,28.50$; HRMS $\left(\mathrm{ESI}^{-}\right)$Calculated for $\mathrm{C}_{48} \mathrm{H}_{48} \mathrm{O}_{7} \mathrm{~N}_{6} \mathrm{Cl}[\mathrm{M}+\mathrm{Cl}]^{-} 855.3278$, found 855.3260. 
S4

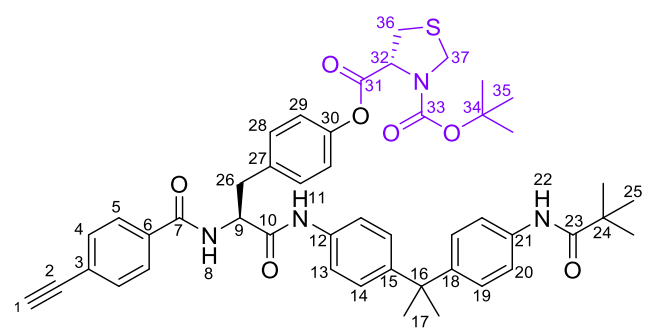

To a solution of barrier $\mathbf{S 3}\left(120 \mathrm{mg}, 0.20 \mathrm{mmol}, 1.0\right.$ equiv.) in $\mathrm{CHCl}_{3}$ :THF (1:3, $\left.9.5 \mathrm{~mL}\right)$ was added Boc-Thi-OH (56 mg, $0.24 \mathrm{mmol}, 1.2$ equiv.), DMAP (2.4 mg, $0.02 \mathrm{mmol}, 0.1$ equiv.) and $\mathrm{EDCl} \cdot \mathrm{HCl}$ (46 mg, $0.24 \mathrm{mmol}, 1.2$ equiv.). The reaction mixture was stirred for $20 \mathrm{~h}$ at room temperature. The solvent was removed under reduced pressure. Purification by flash column chromatography $\left(\mathrm{SiO}_{2}\right.$, EtOAc/hexane 2:3) yielded $\mathbf{S} 4$ as a colorless solid (110 $\mathrm{mg}, 0.13 \mathrm{mmol}$, $67 \%)$.

${ }^{1} \mathrm{H}$ NMR $\left(600 \mathrm{MHz}\right.$, Acetone- $\left.d_{6}\right) \delta 9.41\left(\mathrm{~s}, 1 \mathrm{H}, \mathrm{H}_{11}\right), 8.54\left(\mathrm{~s}, 1 \mathrm{H}, \mathrm{H}_{22}\right), 8.04(\mathrm{~d}, J=8.0 \mathrm{~Hz}, 1 \mathrm{H}$, $\left.\mathrm{H}_{8}\right), 7.91-7.87\left(\mathrm{~m}, 2 \mathrm{H}, \mathrm{H}_{5}\right), 7.61-7.58\left(\mathrm{~m}, 2 \mathrm{H}, \mathrm{H}_{13}\right), 7.58-7.56\left(\mathrm{~m}, 2 \mathrm{H}, \mathrm{H}_{4}\right), 7.56-7.53(\mathrm{~m}$, $\left.2 \mathrm{H}, \mathrm{H}_{20}\right), 7.47-7.41\left(\mathrm{~m}, 2 \mathrm{H}, \mathrm{H}_{28}\right), 7.20-7.17\left(\mathrm{~m}, 2 \mathrm{H}, \mathrm{H}_{19}\right) 7.17-7.15\left(\mathrm{~m}, 2 \mathrm{H}, \mathrm{H}_{14}\right), 7.11-$ $7.05\left(\mathrm{~m}, 2 \mathrm{H}, \mathrm{H}_{29}\right) 5.05-4.98\left(\mathrm{~m}, 1 \mathrm{H}, \mathrm{H}_{9}\right), 4.98-4.94\left(\mathrm{~m}, 1 \mathrm{H}, \mathrm{H}_{32}\right), 4.62(\mathrm{~d}, J=8.8 \mathrm{~Hz}, 1 \mathrm{H}$, $\left.\mathrm{H}_{37}\right), 4.47\left(\mathrm{~d}, J=8.8 \mathrm{~Hz}, 1 \mathrm{H}, \mathrm{H}_{37^{\prime}}\right), 3.83\left(\mathrm{~s}, 1 \mathrm{H}, \mathrm{H}_{1}\right), 3.61\left(\mathrm{t}, J=9.7 \mathrm{~Hz}, 0.5 \mathrm{H}, \mathrm{H}_{36}\right), 3.53(\mathrm{t}, J=$ $\left.9.5 \mathrm{~Hz}, 0.4 \mathrm{H}, \mathrm{H}_{36}\right), 3.49-3.43\left(\mathrm{~m}, 0.5 \mathrm{H}, \mathrm{H}_{36^{\prime}}\right), 3.43-3.36\left(\mathrm{~m}, 0.4 \mathrm{H}, \mathrm{H}_{36^{\prime}}\right), 3.36(\mathrm{dd}, J=14.0$, $\left.5.8 \mathrm{~Hz}, 1 \mathrm{H}, \mathrm{H}_{26}\right), 3.19\left(\mathrm{dd}, J=14.0,8.7 \mathrm{~Hz}, 1 \mathrm{H}, \mathrm{H}_{26}\right), 1.63\left(\mathrm{~s}, 6 \mathrm{H}, \mathrm{H}_{17}\right), 1.50-1.42(\mathrm{~m}, 9 \mathrm{H}$, $\left.\mathrm{H}_{35}+\mathrm{H}_{35}\right), 1.28\left(\mathrm{~s}, 1 \mathrm{H}, \mathrm{H}_{25}\right) \mathrm{ppm} ;{ }^{13} \mathrm{C}$ NMR $\left(151 \mathrm{MHz}\right.$, Acetone- $\left.d_{6}\right) \delta 176.91,170.23,170.10$, $166.75,153.82,153.55,150.47,150.38,147.09,146.37,138.13,137.30,137.21,136.62$, $135.23,132.68,131.31,131.24,128.43,127.78,127.53,126.15,122.14,122.02,120.38$, $120.11,120.02,83.46,83.37,81.27,62.65,62.42,56.74,50.01,48.94,42.72,40.10,37.87$, $35.13,33.72,31.11,28.41,27.75 \mathrm{ppm}$ (some carbon signals appear twice due to the two rotamers of the Boc group); HRMS $\left(\mathrm{ESI}^{+}\right)$Calculated for $\mathrm{C}_{47} \mathrm{H}_{52} \mathrm{O}_{7} \mathrm{~N}_{4} \mathrm{SNa}[\mathrm{M}+\mathrm{Na}]^{+}$839.3449, found 839.3438 . 
5

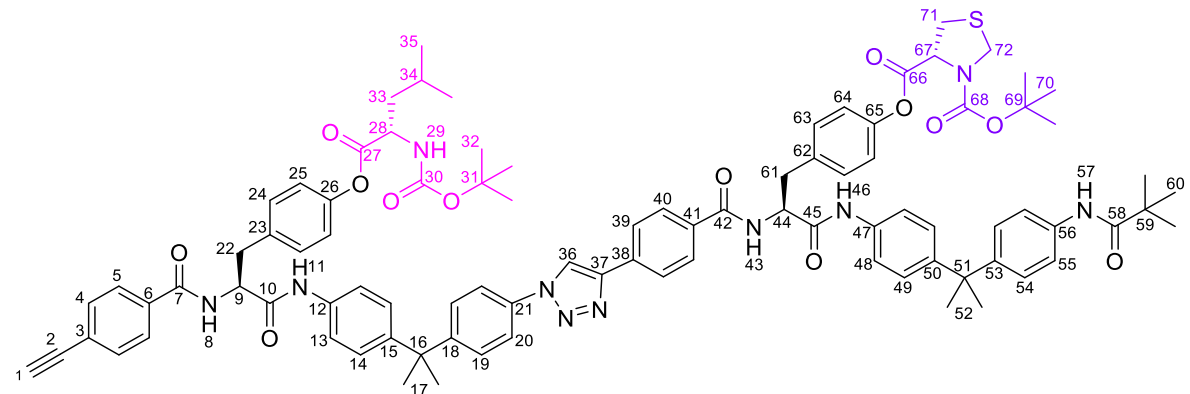

Tentagel $^{\mathrm{TM}} \mathrm{TBTA}$ resin $(0.17 \mathrm{mmol} / \mathrm{g}, 380 \mathrm{mg}, 65 \mu \mathrm{mol}, 0.5$ equiv. $)$ and $\mathrm{Cu}(\mathrm{MeCN})_{4} \mathrm{PF}_{6}(22 \mathrm{mg}$, $59 \mu \mathrm{mol}, 0.45$ equiv.) were added to a sealed vial. The vial was purged by repeated cycles of vacuum evacuation/filling with argon. The mixture was then dissolved in $\mathrm{CH}_{2} \mathrm{Cl}_{2}(2.5 \mathrm{~mL})$ which was sparged with argon prior to use. The mixture was stirred for 10 minutes. To the mixture was added a solution of S5 (112 mg, $130 \mu \mathrm{mol}, 1.0$ equiv.) and S4 (110 mg, $130 \mu \mathrm{mol}, 1.0$ equiv.) in degassed $\mathrm{CH}_{2} \mathrm{Cl}_{2}: \mathrm{tBuOH}(4: 1,4.5 \mathrm{~mL})$. The reaction was stirred for $20 \mathrm{~h}$ at room temperature. The reaction was filtered and washed with $\mathrm{CH}_{2} \mathrm{Cl}_{2}$ (to recover and recycle TBTA resin) and the solvent was removed under reduced pressure. The crude product (222 $\mathrm{mg}$ ) was used directly without further purification.

The crude product was dissolved in DMF $(2.5 \mathrm{~mL})$ and TASF (70 mg, $260 \mu \mathrm{mol}, 2.0$ equiv.) was added. The reaction mixture was stirred at room temperature for 1 minute. The reaction was poured into a stirred mixture of $\mathrm{CH}_{2} \mathrm{Cl}_{2}(20 \mathrm{~mL})$ and saturated aqueous $\mathrm{NH}_{4} \mathrm{Cl}(20 \mathrm{~mL})$. The organic phase was isolated, dried over $\mathrm{Na}_{2} \mathrm{SO}_{4}$ and the solvent removed under reduced pressure. The crude residue was purified by flash column chromatography $\left(\mathrm{SiO}_{2}\right.$, EtOAc/hexane 1:1 to recover unreacted $\mathbf{S} 4(10 \mathrm{mg})$, then $\left.\mathrm{CH}_{2} \mathrm{Cl}_{2} / \mathrm{IPA} 20: 1\right)$ to give $\mathbf{5}$ as a pale brown solid (135 mg, $86 \mu \mathrm{mol}, 66 \%$ over 2 steps).

${ }^{1} \mathrm{H}$ NMR $\left(600 \mathrm{MHz}\right.$, Acetone- $\left.d_{6}\right) \delta 9.50-9.41\left(\mathrm{~m}, 2 \mathrm{H}, \mathrm{H}_{11}+\mathrm{H}_{46}\right), 9.05$ (bs, $\left.1 \mathrm{H}, \mathrm{H}_{36}\right), 8.56$ (s, $\left.1 \mathrm{H}, \mathrm{H}_{57}\right), 8.10-8.01\left(\mathrm{~m}, 4 \mathrm{H}, \mathrm{H}_{8}+\mathrm{H}_{43}+\mathrm{H}_{39}\right), 8.01-7.95\left(\mathrm{~m}, 2 \mathrm{H}, \mathrm{H}_{40}\right), 7.89-7.83\left(\mathrm{~m}, 4 \mathrm{H}, \mathrm{H}_{5}\right.$ $\left.+\mathrm{H}_{20}\right), 7.60-7.56\left(\mathrm{~m}, 4 \mathrm{H}, \mathrm{H}_{13}+\mathrm{H}_{48}\right), 7.56-7.51\left(\mathrm{~m}, 4 \mathrm{H}, \mathrm{H}_{4}+\mathrm{H}_{55}\right), 7.51-7.46\left(\mathrm{~m}, 2 \mathrm{H}, \mathrm{H}_{19}\right)$, $7.46-7.42\left(\mathrm{~m}, 2 \mathrm{H}, \mathrm{H}_{24} / \mathrm{H}_{63}\right), 7.42-7.38\left(\mathrm{~m}, 2 \mathrm{H}, \mathrm{H}_{24} / \mathrm{H}_{63}\right), 7.26-7.20\left(\mathrm{~m}, 2 \mathrm{H}, \mathrm{H}_{14} / \mathrm{H}_{49}\right), 7.19-$ $7.16\left(\mathrm{~m}, 2 \mathrm{H}, \mathrm{H}_{54}\right), 7.15-7.12\left(\mathrm{~m}, 2 \mathrm{H}, \mathrm{H}_{14} / \mathrm{H}_{49}\right), 7.09-7.05\left(\mathrm{~m}, 2 \mathrm{H}, \mathrm{H}_{25} / \mathrm{H}_{64}\right), 7.05-7.00(\mathrm{~m}$, $\left.2 \mathrm{H}, \mathrm{H}_{25} / \mathrm{H}_{64}\right), 6.46\left(\mathrm{~d}, \mathrm{~J}=8.1 \mathrm{~Hz}, 1 \mathrm{H}, \mathrm{H}_{29}\right), 5.08-4.99\left(\mathrm{~m}, 2 \mathrm{H}, \mathrm{H}_{9}+\mathrm{H}_{44}\right), 4.99-4.90(\mathrm{~m}, 1 \mathrm{H}$, $\left.\mathrm{H}_{67}\right), 4.62\left(\mathrm{~d}, J=8.8 \mathrm{~Hz}, 1 \mathrm{H}, \mathrm{H}_{72}\right), 4.47\left(\mathrm{~d}, J=8.4 \mathrm{~Hz}, 1 \mathrm{H}, \mathrm{H}_{72^{\prime}}\right), 4.35(\mathrm{td}, J=9.4,5.2 \mathrm{~Hz}, 1 \mathrm{H}$, $\left.\mathrm{H}_{28}\right), 3.83\left(\mathrm{~s}, 1 \mathrm{H}, \mathrm{H}_{1}\right), 3.62-3.47\left(\mathrm{~m}, 1 \mathrm{H}, \mathrm{H}_{71}\right), 3.46-3.33\left(\mathrm{~m}, 3 \mathrm{H}, \mathrm{H}_{71}+\mathrm{H}_{22}+\mathrm{H}_{61}\right), 3.20$ (td, J $\left.=14.0,8.7 \mathrm{~Hz}, 2 \mathrm{H}, \mathrm{H}_{22^{\prime}}+\mathrm{H}_{61^{\prime}}\right), 1.88-1.80\left(\mathrm{~m}, 1 \mathrm{H}, \mathrm{H}_{34}\right), 1.80-1.74\left(\mathrm{~m}, 2 \mathrm{H}, \mathrm{H}_{33}\right), 1.73(\mathrm{~s}, 6 \mathrm{H}$, $\left.\mathrm{H}_{17} / \mathrm{H}_{52}\right), 1.63\left(\mathrm{~s}, 6 \mathrm{H}, \mathrm{H}_{17} / \mathrm{H}_{52}\right), 1.47-1.41\left(\mathrm{~s}, 4 \mathrm{H}, \mathrm{H}_{70}+5 \mathrm{H}, \mathrm{H}_{70}\right), 1.41-1.38\left(\mathrm{~s}, 9 \mathrm{H}, \mathrm{H}_{32}\right), 1.26$ $\left(\mathrm{s}, 9 \mathrm{H}, \mathrm{H}_{60}\right), 0.98\left(\mathrm{~d}, J=6.7 \mathrm{~Hz}, 3 \mathrm{H}, \mathrm{H}_{35}\right), 0.96\left(\mathrm{~d}, J=6.4 \mathrm{~Hz}, 3 \mathrm{H}, \mathrm{H}_{35}\right) \mathrm{ppm} ;{ }^{13} \mathrm{C}$ NMR $(151$ $\mathrm{MHz}$, Acetone- $\left.d_{6}\right) \delta 176.97,172.70,170.61,170.07,169.80,167.31,166.97,162.76,156.64$, $153.80,153.53,152.47,150.60,150.42,150.33,147.72,147.16,146.38,146.25,138.05$, $137.50,137.19,136.60,136.21,135.71,135.08,134.66,134.64,134.40,134.33,132.65$, 
$131.33,131.25,131.17,128.97,128.95,128.45,127.83,127.78,127.52,126.15,126.10$, 126.08, 122.22, 122.11, 122.00, 120.67, 120.45, 120.34, 120.30, 120.23, 120.21, 83.46, 81.44, $81.27,79.38,62.59,62.40,56.90,56.80,53.39,49.66,48.93,43.20,42.71,40.96,40.08$, 37.90, 37.85, 36.14, 35.12, 33.72, 31.12, 30.94, 28.54, 28.41, 27.76, 25.53, 23.25, 21.75 ppm (some carbon signals appear twice due to the two rotamers of the Boc group); HRMS (ESI ${ }^{+}$) Calculated for $\mathrm{C}_{91} \mathrm{H}_{100} \mathrm{O}_{13} \mathrm{~N}_{10} \mathrm{SNa}[\mathrm{M}+\mathrm{Na}]^{+}$1595.7084, found 1595.7093.

S7

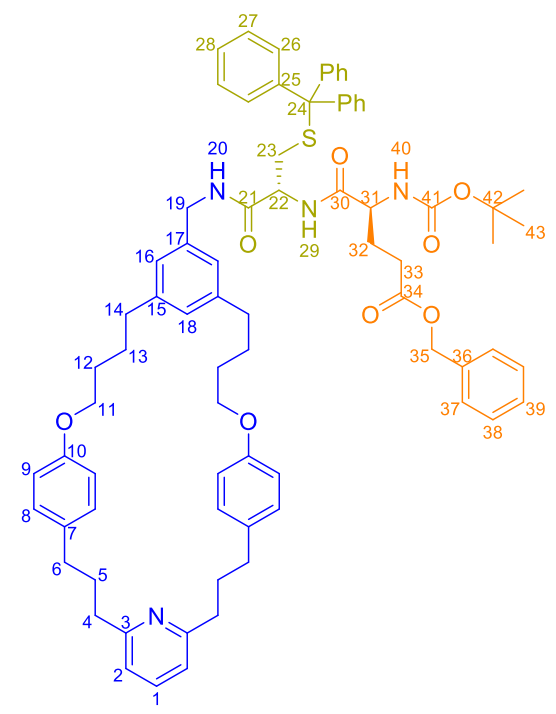

Macrocycle S6 (800 mg, $0.79 \mathrm{mmol}, 1.0$ equiv.) and triphenylmethanol (39 mg, $0.16 \mathrm{mmol}, 0.2$ equiv.) were dissolved in a mixture of $\mathrm{CH}_{2} \mathrm{Cl}_{2}: \mathrm{CF}_{3} \mathrm{CO}_{2} \mathrm{H}(4: 1,32 \mathrm{~mL})$. The solution was stirred at room temperature for 90 min before toluene $(40 \mathrm{~mL})$ was added and the mixture was concentrated to dryness under reduced pressure. The resulting solid was taken up in toluene $(20 \mathrm{~mL})$ and concentrated to dryness under high vacuum to give a foam. The crude was triturated with a mixture of $n$-Hexane/Et ${ }_{2} \mathrm{O}(3: 2)$ twice before being thoroughly dried under high vacuum. The crude salt was dissolved in $\mathrm{CH}_{2} \mathrm{Cl}_{2}$, treated with $\mathrm{Et}_{3} \mathrm{~N}$ (2.5 equiv.) and the solvent was then removed under reduced pressure to afford the crude that was used in the next reaction without further purification.

Boc-Glu(OBzl)-OH (401 mg, $1.19 \mathrm{mmol}, 1.5$ equiv.), EDCl·HCl (235 mg, $1.19 \mathrm{mmol}, 1.5$ equiv.) and $\mathrm{HOBt} \cdot \mathrm{H}_{2} \mathrm{O}$ (24 mg, $0.16 \mathrm{mmol}, 0.2$ equiv.) were added to the crude residue (assume 0.793 mmol, 1.0 equiv.) and the mixture was dissolved in $\mathrm{CH}_{2} \mathrm{Cl}_{2}(16 \mathrm{~mL})$ and stirred at room temperature for $18 \mathrm{~h}$. The mixture was washed with saturated aqueous $\mathrm{NaHCO}_{3}$ and then with brine. The collected organic phase was dried over $\mathrm{Na}_{2} \mathrm{SO}_{4}$, filtered, and the solvent was reduced under reduced pressure to give an off-white foam crude, that was purified by flash column chromatography $\left(\mathrm{SiO}_{2}, \mathrm{CH}_{2} \mathrm{Cl}_{2} / \mathrm{Et}_{2} \mathrm{O} / \mathrm{MeOH} 50: 7: 1\right)$ to give $\mathbf{S 7}$ as a colorless amorphous solid (663 mg, $0.54 \mathrm{mmol}, 68 \%$ ). 
${ }^{1} \mathrm{H}$ NMR $\left(600 \mathrm{MHz}, \mathrm{DMSO}-d_{6}\right) \delta 8.45\left(\mathrm{t}, J=6.1 \mathrm{~Hz}, 1 \mathrm{H}, \mathrm{H}_{20}\right), 7.99\left(\mathrm{~d}, J=8.0 \mathrm{~Hz}, 1 \mathrm{H}, \mathrm{H}_{29}\right)$, $7.58\left(\mathrm{t}, J=7.6 \mathrm{~Hz}, 1 \mathrm{H}, \mathrm{H}_{1}\right), 7.36-7.26\left(\mathrm{~m}, 17 \mathrm{H}, \mathrm{H}_{26}+\mathrm{H}_{27}+\mathrm{H}_{37}+\mathrm{H}_{38}+\mathrm{H}_{39}\right), 7.24-7.20(\mathrm{~m}$, $\left.3 \mathrm{H}, \mathrm{H}_{28}\right), 7.11\left(\mathrm{~d}, J=7.6 \mathrm{~Hz}, 1 \mathrm{H}, \mathrm{H}_{40}\right), 7.06\left(\mathrm{~d}, J=7.6 \mathrm{~Hz}, 2 \mathrm{H}, \mathrm{H}_{2}\right), 7.02(\mathrm{~d}, J=8.6 \mathrm{~Hz}, 4 \mathrm{H}$, $\left.\mathrm{H}_{9}\right), 6.92\left(\mathrm{~s}, 1 \mathrm{H}, \mathrm{H}_{18}\right), 6.85\left(\mathrm{~s}, 2 \mathrm{H}, \mathrm{H}_{16}\right), 6.72\left(\mathrm{~d}, J=8.5 \mathrm{~Hz}, 4 \mathrm{H}, \mathrm{H}_{8}\right), 5.06\left(\mathrm{~s}, 2 \mathrm{H}, \mathrm{H}_{35}\right), 4.37$ (d, $\left.J=7.2 \mathrm{~Hz}, 1 \mathrm{H}, \mathrm{H}_{22}\right), 4.29$ (dd, $\left.J=15.5,6.3 \mathrm{~Hz}, 1 \mathrm{H}, \mathrm{H}_{19}\right), 4.12\left(\mathrm{dd}, J=15.5,5.6 \mathrm{~Hz}, 1 \mathrm{H}, \mathrm{H}_{19^{\prime}}\right.$ ), $3.94\left(\mathrm{q}, J=7.5 \mathrm{~Hz}, 1 \mathrm{H}, \mathrm{H}_{31}\right.$ ), $3.84\left(\mathrm{t}, J=5.9 \mathrm{~Hz}, 4 \mathrm{H}, \mathrm{H}_{11}\right), 2.69\left(\mathrm{t}, J=7.3 \mathrm{~Hz}, 4 \mathrm{H}, \mathrm{H}_{4}\right), 2.49-$ $2.44\left(\mathrm{~m}, 8 \mathrm{H}, \mathrm{H}_{6}+\mathrm{H}_{14}\right), 2.41-2.36\left(\mathrm{~m}, 4 \mathrm{H}, \mathrm{H}_{23}+\mathrm{H}_{33}\right), 1.97-1.88\left(\mathrm{~m}, 5 \mathrm{H}, \mathrm{H}_{5}+\mathrm{H}_{32}\right), 1.82-$ $1.76\left(\mathrm{~m}, 1 \mathrm{H}, \mathrm{H}_{32}\right), 1.67-1.55\left(\mathrm{~m}, 8 \mathrm{H}, \mathrm{H}_{12}+\mathrm{H}_{13}\right), 1.33\left(\mathrm{~s}, 9 \mathrm{H}, \mathrm{H}_{43}\right) ;{ }^{13} \mathrm{C}$ NMR $(151 \mathrm{MHz}, \mathrm{DMSO}-$ $\left.d_{6}\right) \delta 172.2,171.3,169.4,160.6,156.8,144.2,141.8,138.7,136.7,136.2,133.9,129.2,129.0$, $128.4,128.04,127.95,127.8,126.8,126.6,124.7,120.2,114.1,78.4,67.0,65.8,65.4,53.7$, 51.5, 42.1, 40.0, 36.6, 34.4, 34.0, 33.7, 31.4, 30.1, 28.1, 27.9, 27.6, 27.0; HRMS (ESI $\left.{ }^{+}\right)$ Calculated for $\mathrm{C}_{77} \mathrm{H}_{87} \mathrm{~N}_{4} \mathrm{O}_{8} \mathrm{~S}[\mathrm{M}+\mathrm{H}]^{+}$1227.6239, found 1227.6231 .

S8

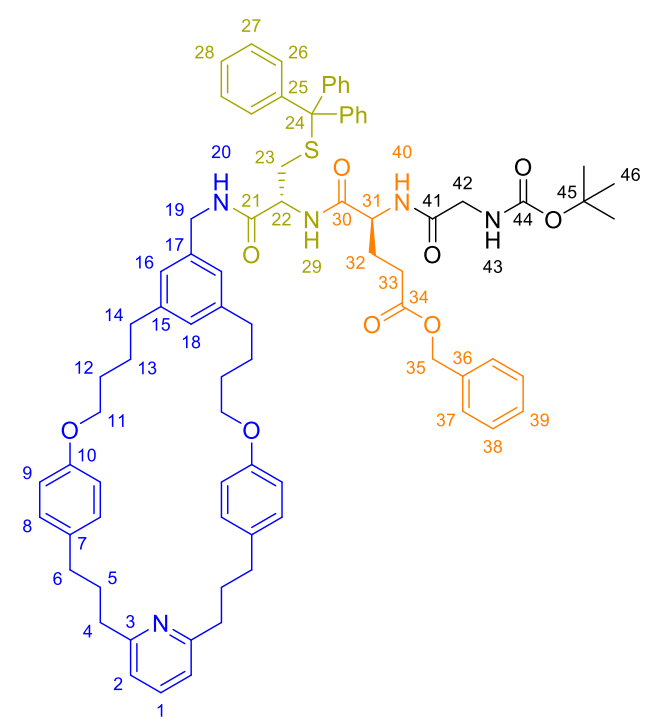

Macrocycle S7 (630 mg, $0.51 \mathrm{mmol}, 1.0$ equiv.) and triphenylmethanol ( $25 \mathrm{mg}, 0.10 \mathrm{mmol}, 0.2$ equiv.) were dissolved in a mixture of $\mathrm{CH}_{2} \mathrm{Cl}_{2}: \mathrm{CF}_{3} \mathrm{CO}_{2} \mathrm{H}(4: 1,20 \mathrm{~mL})$. The solution was stirred at room temperature for $90 \mathrm{~min}$ before toluene $(40 \mathrm{~mL})$ was added and the mixture was concentrated to dryness under reduced pressure to give a foam. The crude salt was dissolved in $\mathrm{CH}_{2} \mathrm{Cl}_{2}$ and washed with saturated aqueous $\mathrm{NaHCO}_{3}$ and brine. The collected organic phase was dried over $\mathrm{Na}_{2} \mathrm{SO}_{4}$, filtered, and the solvent was reduced under reduced pressure to afford the crude as a yellow oil that was used in the next reaction without further purification. Boc-Gly-OH (132 mg, $0.76 \mathrm{mmol}, 1.5$ equiv.), EDCl. $\mathrm{HCl}(150 \mathrm{mg}, 0.76 \mathrm{mmol}, 1.5$ equiv.) and $\mathrm{HOBt} \cdot \mathrm{H}_{2} \mathrm{O}$ (16 mg, $0.10 \mathrm{mmol}, 0.2$ equiv.) were added to the crude residue (assume 0.51 mmol, 1.0 equiv.) and the mixture was dissolved in $\mathrm{CH}_{2} \mathrm{Cl}_{2}(10 \mathrm{~mL})$ and stirred at room temperature for $18 \mathrm{~h}$. The crude was purified by flash column chromatography $\left(\mathrm{SiO}_{2}\right.$, $\mathrm{CH}_{2} \mathrm{Cl}_{2} / \mathrm{MeOH} 97: 3$ ) to yield $\mathbf{S} 8$ as a colorless amorphous solid (605 mg, $0.47 \mathrm{mmol}, 92 \%$ ). 
${ }^{1} \mathrm{H}$ NMR $\left(600 \mathrm{MHz}, \mathrm{DMSO}-d_{6}\right) \delta 8.41\left(\mathrm{t}, J=6.1 \mathrm{~Hz}, 1 \mathrm{H}, \mathrm{H}_{20}\right), 8.26\left(\mathrm{~d}, J=8.1 \mathrm{~Hz}, 1 \mathrm{H}, \mathrm{H}_{29}\right)$, $7.92\left(\mathrm{~d}, J=7.8 \mathrm{~Hz}, 1 \mathrm{H}, \mathrm{H}_{40}\right), 7.58\left(\mathrm{t}, J=7.6 \mathrm{~Hz}, 1 \mathrm{H}, \mathrm{H}_{1}\right), 7.36-7.26\left(\mathrm{~m}, 17 \mathrm{H}, \mathrm{H}_{26}+\mathrm{H}_{27}+\mathrm{H}_{37}\right.$ $\left.+\mathrm{H}_{38}+\mathrm{H}_{39}\right), 7.25-7.20\left(\mathrm{~m}, 3 \mathrm{H}, \mathrm{H}_{28}\right), 7.06\left(\mathrm{~d}, J=7.6 \mathrm{~Hz}, 2 \mathrm{H}, \mathrm{H}_{2}\right), 7.02\left(\mathrm{~d}, J=8.4 \mathrm{~Hz}, 4 \mathrm{H}, \mathrm{H}_{8}\right)$, $6.99\left(\mathrm{t}, J=5.9 \mathrm{~Hz}, 1 \mathrm{H}, \mathrm{H}_{43}\right), 6.91\left(\mathrm{~s}, 1 \mathrm{H}, \mathrm{H}_{18}\right), 6.85\left(\mathrm{~d}, J=1.5 \mathrm{~Hz}, 2 \mathrm{H}, \mathrm{H}_{16}\right), 6.72(\mathrm{~d}, J=8.6$ $\left.\mathrm{Hz}, 4 \mathrm{H}, \mathrm{H}_{9}\right), 5.05\left(\mathrm{~s}, 2 \mathrm{H}, \mathrm{H}_{35}\right), 4.41-4.26\left(\mathrm{~m}, 3 \mathrm{H}, \mathrm{H}_{19}+\mathrm{H}_{22}+\mathrm{H}_{31}\right), 4.11$ (dd, J = 15.5, 5.6 Hz, $\left.1 \mathrm{H}, \mathrm{H}_{19}\right), 3.84\left(\mathrm{t}, J=5.9 \mathrm{~Hz}, 4 \mathrm{H}, \mathrm{H}_{11}\right), 3.59-3.51\left(\mathrm{~m}, 2 \mathrm{H}, \mathrm{H}_{42}\right), 2.69\left(\mathrm{t}, J=7.3 \mathrm{~Hz}, 4 \mathrm{H}, \mathrm{H}_{4}\right)$, $2.49-2.45\left(\mathrm{~m}, 8 \mathrm{H}, \mathrm{H}_{6}+\mathrm{H}_{14}\right), 2.44-2.32\left(\mathrm{~m}, 4 \mathrm{H}, \mathrm{H}_{23}+\mathrm{H}_{33}\right), 1.96-1.88\left(\mathrm{~m}, 5 \mathrm{H}, \mathrm{H}_{5}+\mathrm{H}_{32}\right)$, $1.84-1.73\left(\mathrm{~m}, 1 \mathrm{H}, \mathrm{H}_{32}\right), 1.67-1.56\left(\mathrm{~m}, 8 \mathrm{H}, \mathrm{H}_{12}+\mathrm{H}_{13}\right), 1.34\left(\mathrm{~s}, 9 \mathrm{H}, \mathrm{H}_{46}\right) ;{ }^{13} \mathrm{C}$ NMR $(151 \mathrm{MHz}$, DMSO- $\left.d_{6}\right) \delta 172.2,170.7,169.5,169.4,160.6,156.8,155.8,144.2,141.8,138.8,136.7,136.1$, $133.9,129.2,129.1,128.4,128.1,128.0,127.9,126.8,126.6,124.7,120.2,114.1,78.1,67.0$, 65.9, 65.5, 51.8, 43.3, 42.1, 40.1, 36.6, 34.41, 34.36, 33.7, 31.4, 29.8, 28.2, 27.9, 27.7, 27.6; HRMS $\left(\mathrm{ESI}^{+}\right)$Calculated for $\mathrm{C}_{79} \mathrm{H}_{90} \mathrm{~N}_{5} \mathrm{O}_{9} \mathrm{~S}[\mathrm{M}+\mathrm{H}]^{+} 1284.6454$, found 1284.6454 .

3

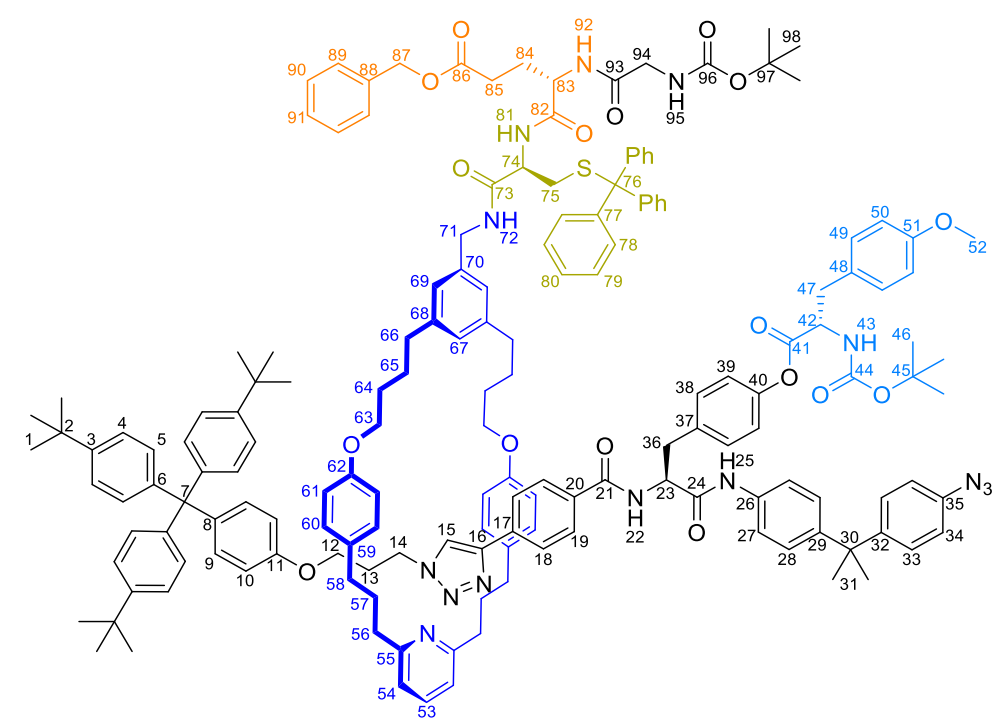

Macrocycle S8 (94 mg, $73 \mu \mathrm{mol}, 1.5$ equiv.) and $\mathrm{Cu}(\mathrm{MeCN})_{4} \mathrm{PF}_{6}(9 \mathrm{mg}, 24 \mu \mathrm{mol}, 0.5$ equiv.) were dissolved in $\mathrm{CH}_{2} \mathrm{Cl}_{2}(1.5 \mathrm{~mL})$ and the resulting solution was both degassed and concentrated by approximately half via nitrogen sparging. The solution was added to azide

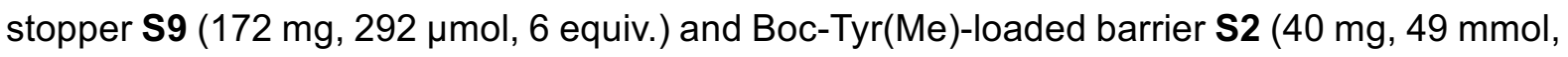
1 equiv.) in $\mathrm{CH}_{2} \mathrm{Cl}_{2}(0.7 \mathrm{~mL})$ with $\mathrm{CH}_{2} \mathrm{Cl}_{2}(2 \times 0.4 \mathrm{~mL})$ being used as a transfer wash. The resulting mixture was further degassed via nitrogen sparging to give a total reaction volume of ca. $1.5 \mathrm{~mL}$. $t$-BuOH ( 3 drops) was added and the solution stirred at room temperature for $24 \mathrm{~h}$. The resulting solution was concentrated to dryness under reduced pressure. The crude residue was purified by flash column chromatography $\left(\mathrm{SiO}_{2}\right.$, Hexane/EtOAc 4:1 (to collect 150 $\mathrm{mg}$ of unreacted stopper S9), then Hexane/EtOAc 2:1 (to collect $20 \mathrm{mg}$ of free thread S10), then EtOAc (to collect $130 \mathrm{mg}$ of a mixture of rotaxane product 3 and macrocycle $\mathbf{S} 8$ ). This 
last fraction (130 mg) was collected and further purified by size exclusion chromatography (SX-1 beads, $\mathrm{CH}_{2} \mathrm{Cl}_{2}$ ) to afford a fraction containing rotaxane $3(44 \mathrm{mg}$ ) and a small amount of impurities. The fraction was washed with saturated aqueous $\mathrm{Na}_{4}$ EDTA solution, extracted with $\mathrm{CH}_{2} \mathrm{Cl}_{2}$ and further purified by preparative scale $\operatorname{TLC}\left(\mathrm{SiO}_{2}, 1000 \mu \mathrm{m}\right.$ Merck plates, eluting twice in Hexane/EtOAc 5:4) to afford 3 as an off-yellow solid (25 mg, $9 \mu \mathrm{mol}, 19 \%$ ).

${ }^{1} \mathrm{H}$ NMR $\left(600 \mathrm{MHz}\right.$, Acetone- $\left.d_{6}\right) \delta 9.50\left(\mathrm{~s}, 1 \mathrm{H}, \mathrm{H}_{25}\right), 8.11\left(\mathrm{~d}, J=7.9 \mathrm{~Hz}, 1 \mathrm{H}, \mathrm{H}_{22}\right), 7.85-7.82$ $\left(\mathrm{m}, 2 \mathrm{H}, \mathrm{H}_{81}+\mathrm{H}_{15}\right), 7.81-7.78\left(\mathrm{~m}, 1 \mathrm{H}, \mathrm{H}_{72}\right), 7.76\left(\mathrm{~d}, J=8.0 \mathrm{~Hz}, 2 \mathrm{H}, \mathrm{H}_{19}\right), 7.68(\mathrm{~d}, J=7.1 \mathrm{~Hz}$, $\left.1 \mathrm{H}, \mathrm{H}_{92}\right), 7.62\left(\mathrm{~d}, J=8.0 \mathrm{~Hz}, 2 \mathrm{H}, \mathrm{H}_{18}\right), 7.57\left(\mathrm{~d}, J=8.7 \mathrm{~Hz}, 2 \mathrm{H}, \mathrm{H}_{27}\right), 7.53(\mathrm{t}, J=7.7 \mathrm{~Hz}, 1 \mathrm{H}$, $\left.\mathrm{H}_{53}\right), 7.40-7.35\left(\mathrm{~m}, 8 \mathrm{H}, \mathrm{H}_{38}+\mathrm{H}_{78}\right), 7.35-7.31\left(\mathrm{~m}, 5 \mathrm{H}, \mathrm{H}_{89}+\mathrm{H}_{90}+\mathrm{H}_{91}\right), 7.31-7.27(\mathrm{~m}, 6 \mathrm{H}$, $\left.\mathrm{H}_{4}\right), 7.27-7.22\left(\mathrm{~m}, 10 \mathrm{H}, \mathrm{H}_{33}+\mathrm{H}_{49}+\mathrm{H}_{79}\right), 7.20-7.15\left(\mathrm{~m}, 5 \mathrm{H}, \mathrm{H}_{28}+\mathrm{H}_{80}\right), 7.14-7.10(\mathrm{~m}, 6 \mathrm{H}$, $\left.\mathrm{H}_{5}\right), 7.02\left(\mathrm{~d}, J=7.7 \mathrm{~Hz}, 2 \mathrm{H}, \mathrm{H}_{54}\right), 7.00-6.93\left(\mathrm{~m}, 9 \mathrm{H}, \mathrm{H}_{9}+\mathrm{H}_{39}+\mathrm{H}_{34}+\mathrm{H}_{67}+\mathrm{H}_{69}\right), 6.86(\mathrm{~d}, J=$ $\left.8.5 \mathrm{~Hz}, 2 \mathrm{H}, \mathrm{H}_{50}\right), 6.82-6.76\left(\mathrm{~m}, 4 \mathrm{H}, \mathrm{H}_{60}\right), 6.53\left(\mathrm{~d}, J=8.8 \mathrm{~Hz}, 2 \mathrm{H}, \mathrm{H}_{10}\right), 6.43(\mathrm{~d}, J=8.6 \mathrm{~Hz}$, $\left.2 \mathrm{H}, \mathrm{H}_{61}\right), 6.41\left(\mathrm{~d}, J=8.5 \mathrm{~Hz}, 2 \mathrm{H}, \mathrm{H}_{61}\right), 6.37-6.32\left(\mathrm{~m}, 2 \mathrm{H}, \mathrm{H}_{43}+\mathrm{H}_{95}\right), 5.10-5.03\left(\mathrm{~m}, 3 \mathrm{H}, \mathrm{H}_{23}\right.$ $\left.+\mathrm{H}_{87}\right), 4.61-4.56\left(\mathrm{~m}, 1 \mathrm{H}, \mathrm{H}_{74}\right), 4.55-4.50\left(\mathrm{~m}, 2 \mathrm{H}, \mathrm{H}_{42}+\mathrm{H}_{83}\right), 4.41(\mathrm{dd}, J=15.2,6.3 \mathrm{~Hz}, 1 \mathrm{H}$, $\left.\mathrm{H}_{71}\right), 4.25$ (dd, $\left.J=15.2,5.6 \mathrm{~Hz}, 1 \mathrm{H}, \mathrm{H}_{71^{\prime}}\right), 4.03\left(\mathrm{t}, J=7.1 \mathrm{~Hz}, 2 \mathrm{H}, \mathrm{H}_{14}\right), 3.75\left(\mathrm{~s}, 3 \mathrm{H}, \mathrm{H}_{52}\right), 3.73$ $-3.67\left(\mathrm{~m}, 6 \mathrm{H}, \mathrm{H}_{94}+\mathrm{H}_{63}\right), 3.44\left(\mathrm{t}, J=6.0 \mathrm{~Hz}, 2 \mathrm{H}, \mathrm{H}_{12}\right), 3.30$ (dd, $\left.J=13.8,6.3 \mathrm{~Hz}, 1 \mathrm{H}, \mathrm{H}_{36}\right), 3.23$ $-3.13\left(\mathrm{~m}, 2 \mathrm{H}, \mathrm{H}_{36^{\prime}}+\mathrm{H}_{47}\right), 3.12-3.04\left(\mathrm{~m}, 1 \mathrm{H}, \mathrm{H}_{47^{\prime}}\right), 2.71\left(\mathrm{dd}, J=12.1,5.4 \mathrm{~Hz}, 1 \mathrm{H}, \mathrm{H}_{75}\right), 2.67$ $-2.57\left(\mathrm{~m}, 5 \mathrm{H}, \mathrm{H}_{75}+\mathrm{H}_{56}\right), 2.56-2.46\left(\mathrm{~m}, 10 \mathrm{H}, \mathrm{H}_{85}+\mathrm{H}_{66}+\mathrm{H}_{58}\right), 2.19(\mathrm{dd}, J=13.8,7.8 \mathrm{~Hz}, 1 \mathrm{H}$, $\mathrm{H}_{84}$ ), $2.00-1.98$ (signal hidden under the solvent peak, $\left.1 \mathrm{H}, \mathrm{H}_{84}\right), 1.90-1.82\left(\mathrm{~m}, 6 \mathrm{H}, \mathrm{H}_{13}+\right.$ $\left.\mathrm{H}_{57}\right), 1.73-1.67\left(\mathrm{~m}, 4 \mathrm{H}, \mathrm{H}_{65}\right), 1.66-1.61\left(\mathrm{~s}, 10 \mathrm{H}, \mathrm{H}_{31}, \mathrm{H}_{64}\right), 1.38\left(\mathrm{~s}, 9 \mathrm{H}, \mathrm{H}_{46} / \mathrm{H}_{98}\right), 1.36(\mathrm{~s}, 9 \mathrm{H}$, $\left.\mathrm{H}_{46} / \mathrm{H}_{98}\right), 1.29\left(\mathrm{~s}, 27 \mathrm{H}, \mathrm{H}_{1}\right) ;{ }^{13} \mathrm{C}$ NMR $\left(151 \mathrm{MHz}\right.$, Acetone- $\left.d_{6}\right) \delta 173.32,171.90,171.67,171.12$, $170.60,170.41,167.40,162.50,159.66,158.04,157.32,157.18,156.34,150.52,149.10$, $148.78,146.84,146.55,145.63,145.33,143.59,140.21,139.65,138.08,137.55,137.48$, $137.40,136.32,135.09,134.30,133.52,132.65,131.45,131.26,130.34,130.01,129.77$, $129.29,129.18,128.90,128.83,128.81,128.76,127.84,127.70,127.62,125.98,125.79$, $125.06,122.25,122.06,120.59,120.36,119.42$, 115.00, 114.99, 114.68, 114.17, 79.74, 79.55, $68.13,67.37,66.63,64.76,63.90,56.83,56.66,55.48,54.96,53.86,53.50,47.48,44.99$, $43.67,42.91,38.44,38.00,37.29,36.21,35.62,35.04,34.87,32.98,31.69,31.03,30.87$, 29.14, 28.67, 28.55; HRMS $\left(\mathrm{ESI}^{+}\right)$Calculated for $\mathrm{C}_{167} \mathrm{H}_{187} \mathrm{O}_{17} \mathrm{~N}_{14} \mathrm{SNa}[\mathrm{M}+\mathrm{Na}+\mathrm{H}]^{2+} 1357.6903$, found 1357.6962 . 
S10

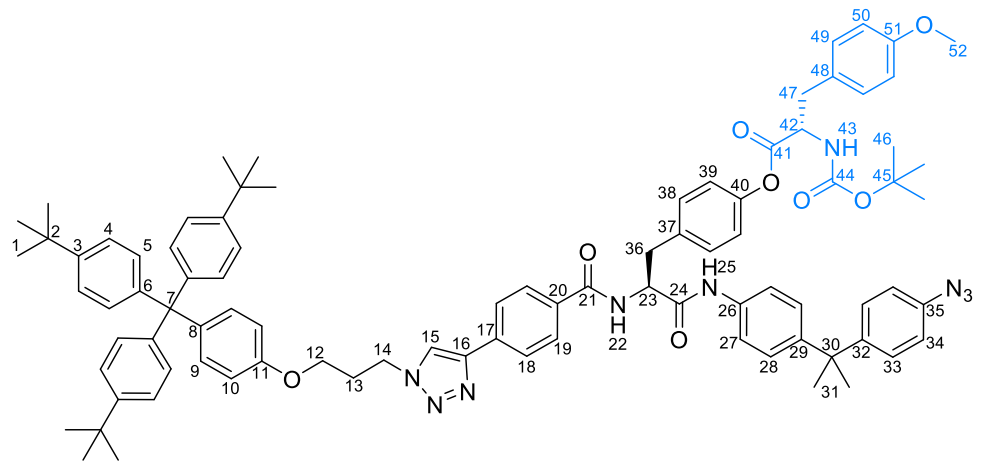

${ }^{1} \mathrm{H}$ NMR $\left(600 \mathrm{MHz}\right.$, Acetone- $d_{6} \delta 9.50\left(\mathrm{~d}, \mathrm{~J}=3.9 \mathrm{~Hz}, 1 \mathrm{H}, \mathrm{H}_{25}\right), 8.48\left(\mathrm{~s}, 1 \mathrm{H}, \mathrm{H}_{15}\right), 8.07(\mathrm{~d}, \mathrm{~J}=$ $\left.7.9 \mathrm{~Hz}, 1 \mathrm{H}, \mathrm{H}_{22}\right), 7.96-7.92\left(\mathrm{~m}, 4 \mathrm{H}, \mathrm{H}_{18}+\mathrm{H}_{19}\right), 7.56\left(\mathrm{~d}, J=8.7 \mathrm{~Hz}, 2 \mathrm{H}, \mathrm{H}_{27}\right), 7.42-7.39(\mathrm{~m}$, $\left.2 \mathrm{H}, \mathrm{H}_{38}\right), 7.31\left(\mathrm{~d}, J=8.6 \mathrm{~Hz}, 6 \mathrm{H}, \mathrm{H}_{4}\right), 7.29-7.26\left(\mathrm{~m}, 2 \mathrm{H}, \mathrm{H}_{34}\right), 7.25\left(\mathrm{~d}, J=8.2 \mathrm{~Hz}, 2 \mathrm{H}, \mathrm{H}_{49}\right)$, $7.17\left(\mathrm{~d}, J=8.7 \mathrm{~Hz}, 2 \mathrm{H}, \mathrm{H}_{28}\right), 7.12\left(\mathrm{~d}, J=8.6 \mathrm{~Hz}, 6 \mathrm{H}, \mathrm{H}_{5}\right), 7.10-7.07\left(\mathrm{~m}, 2 \mathrm{H}, \mathrm{H}_{9}\right), 6.98(\mathrm{~d}, J=$ $\left.8.6 \mathrm{~Hz}, 2 \mathrm{H}, \mathrm{H}_{33}\right), 6.97-6.95\left(\mathrm{~m}, 2 \mathrm{H}, \mathrm{H}_{39}\right), 6.87\left(\mathrm{dd}, J=8.6,1.2 \mathrm{~Hz}, 2 \mathrm{H}, \mathrm{H}_{50}\right), 6.84(\mathrm{~d}, J=9.0$ $\left.\mathrm{Hz}, 2 \mathrm{H}, \mathrm{H}_{10}\right), 6.36\left(\mathrm{~d}, J=7.8 \mathrm{~Hz}, 1 \mathrm{H}, \mathrm{H}_{43}\right), 5.01$ (td, $\left.J=8.2,5.8 \mathrm{~Hz}, 1 \mathrm{H}, \mathrm{H}_{23}\right), 4.69$ (t, J = 6.9 $\left.\mathrm{Hz}, 2 \mathrm{H}, \mathrm{H}_{14}\right), 4.51$ (q, J = $7.8 \mathrm{~Hz}, 1 \mathrm{H}, \mathrm{H}_{42}$ ), 4.07 (t, J = 5.9 Hz, 2H, $\mathrm{H}_{12}$ ), $3.75\left(\mathrm{~s}, 3 \mathrm{H}, \mathrm{H}_{52}\right.$ ), 3.35 $\left(\mathrm{dd}, J=13.9,5.8 \mathrm{~Hz}, 1 \mathrm{H}, \mathrm{H}_{36}\right), 3.24-3.14\left(\mathrm{~m}, 2 \mathrm{H}, \mathrm{H}_{36}+\mathrm{H}_{47}\right), 3.08(\mathrm{dd}, J=13.9,8.5 \mathrm{~Hz}, 1 \mathrm{H}$, $\mathrm{H}_{47^{\prime}}$ ), $2.45\left(\mathrm{t}, J=6.4 \mathrm{~Hz}, 2 \mathrm{H}, \mathrm{H}_{13}\right), 1.65\left(\mathrm{~s}, 6 \mathrm{H}, \mathrm{H}_{31}\right), 1.37\left(\mathrm{~s}, 9 \mathrm{H}, \mathrm{H}_{46}\right), 1.29\left(\mathrm{~s}, 27 \mathrm{H}, \mathrm{H}_{1}\right) ;{ }^{13} \mathrm{C}$ NMR $\left(151 \mathrm{MHz}\right.$, Acetone- $\left.d_{6}\right) \delta 171.65,170.47,167.18,159.63,157.62,156.32,150.50$, $149.14,148.74,146.96,146.48,145.22,140.46,138.08,137.55,136.42,135.24,134.14$, $132.79,131.39,131.23,131.16,129.75,129.15,128.88,127.75,125.89,125.06,122.49$, $122.23,120.20,119.40,114.65,114.08,79.52,65.23,63.88,56.83,56.68,55.45,47.91,42.88$, $37.83,37.24,34.85,32.63,31.64,31.00,30.80,30.35,28.51,23.32,14.35$ ppm; HRMS $\left(\mathrm{ESI}^{+}\right)$ Calculated for $\mathrm{C}_{88} \mathrm{H}_{97} \mathrm{O}_{8} \mathrm{~N}_{9} \mathrm{Na}[\mathrm{M}+\mathrm{Na}]^{+} 1430.7352$, found 1430.7291 . 
SI Echavarren et al, 'Sequence-Selective Decapeptide Synthesis by the Parallel Operation of ...'

A.

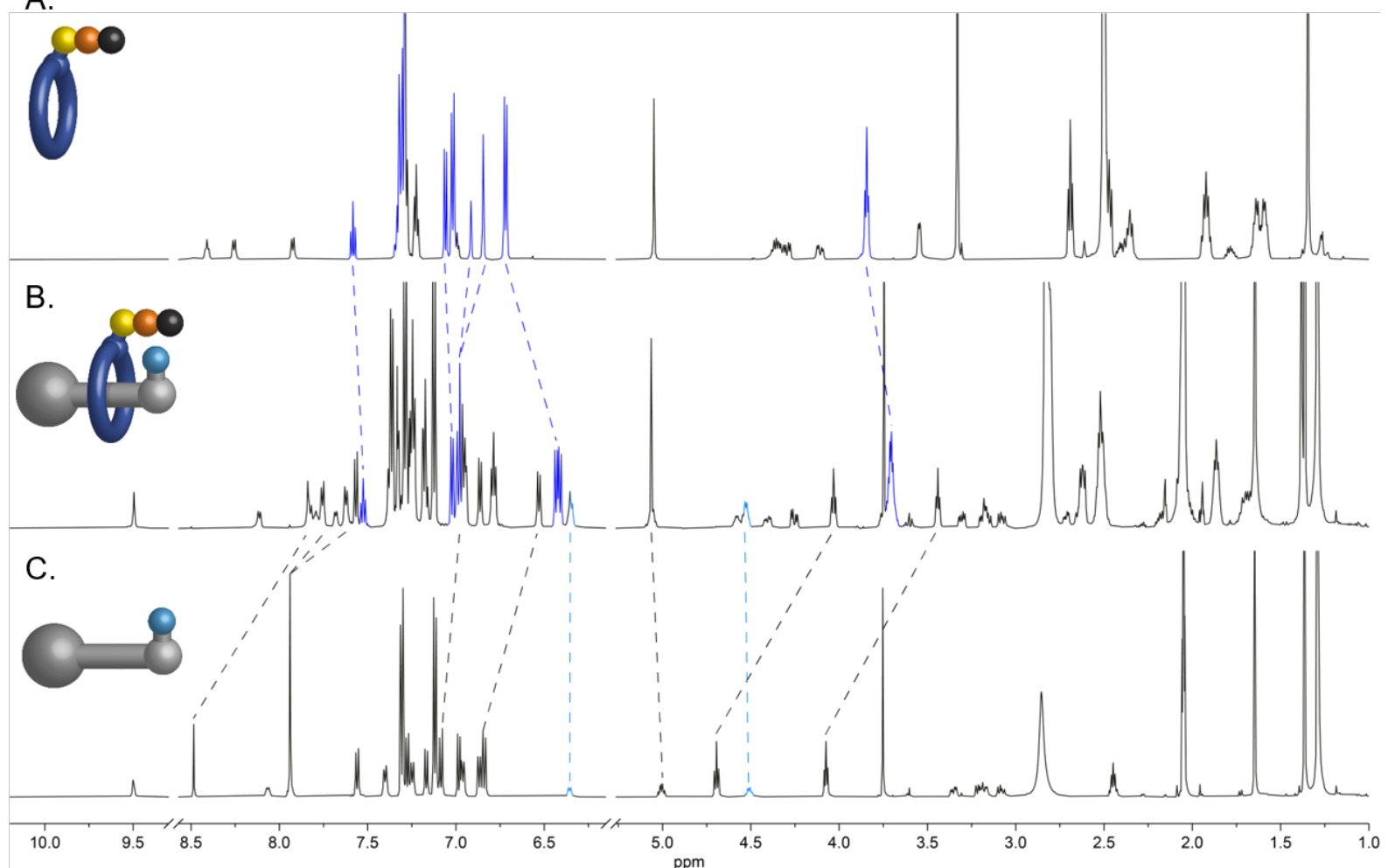

Figure S1. Partial ${ }^{1} \mathrm{H}$ NMR stackplot of (A) Amide macrocycle S8 (600 MHz, DMSO- $\left.d_{6}, 298 \mathrm{~K}\right)$, (B) 1Barrier rotaxane $3\left(600 \mathrm{MHz}\right.$, Acetone- $\left.d_{6}, 298 \mathrm{~K}\right)$ and $(\mathrm{C})$ Isolated non-interlocked free thread $\mathbf{S} 10$ (600 $\mathrm{MHz}$, Acetone- $\left.d_{6}, 298 \mathrm{~K}\right)$. 
1

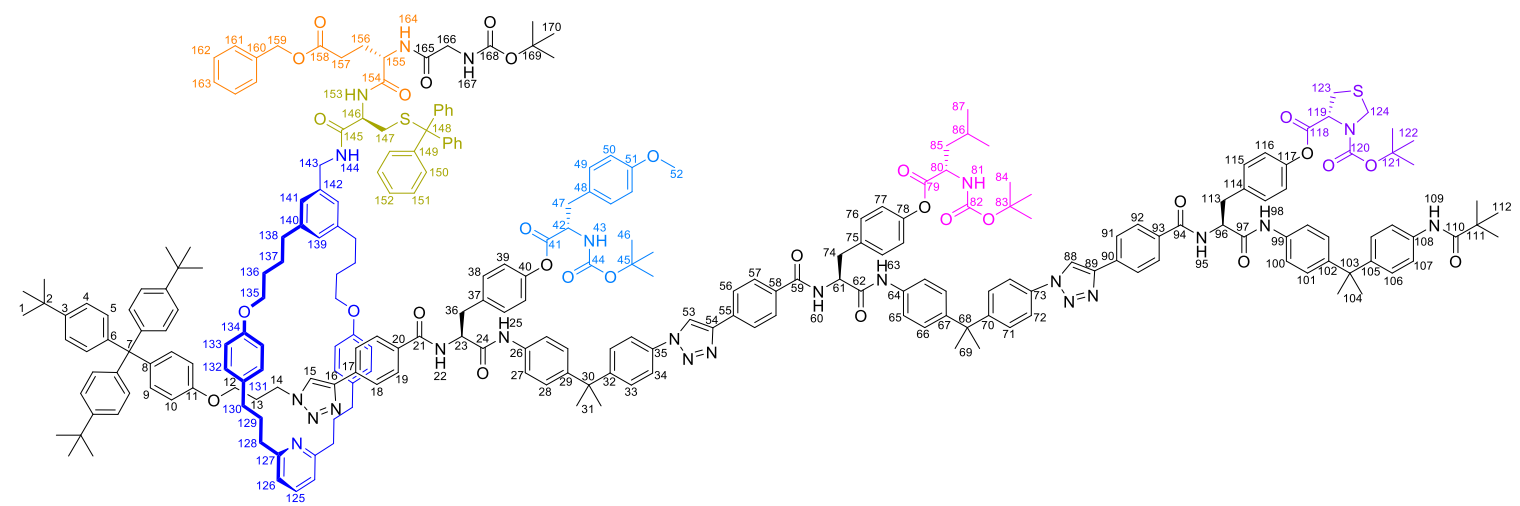

Tentagel $^{\mathrm{TM}}$ TBTA resin (164 mg, $28 \mu \mathrm{mol}, 3.0$ equiv.) and $\mathrm{Cu}(\mathrm{MeCN})_{4} \mathrm{PF}_{6}(5 \mathrm{mg}, 14 \mu \mathrm{mol}, 1.5$ equiv.) were added to a sealed microwave vial. The vial was purged by repeated cycles of vacuum evacuation/filling with argon. The mixture was suspended in $\mathrm{CH}_{2} \mathrm{Cl}_{2}(1.5 \mathrm{~mL})$ which was sparged with argon prior to use. The mixture was stirred for 10 minutes. To the mixture

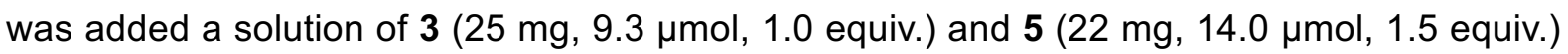
in a degassed mixture of $\mathrm{CH}_{2} \mathrm{Cl}_{2}(0.8 \mathrm{~mL})$ and $t-\mathrm{BuOH}(0.5 \mathrm{~mL})$. The reaction was stirred for 48 hours at room temperature. The reaction was filtered (to recover and recycle TBTA resin) and the solvent removed under reduced pressure. The crude residue was purified by prep. TLC $\left(\mathrm{SiO}_{2}\right.$ (Merck 1000), two times: first in $\mathrm{MeOH}: \mathrm{CH}_{2} \mathrm{Cl}_{2}$ 1:50, second time in $\mathrm{MeOH}: \mathrm{CH}_{2} \mathrm{Cl}_{2}$ $1: 30)$ to give 1 as a glassy solid (25 $\mathrm{mg}, 5.9 \mu \mathrm{mol}, 63 \%)$.

${ }^{1} \mathrm{H}$ NMR $\left(600 \mathrm{MHz}\right.$, Acetone- $\left.d_{6}\right) \delta 9.59-9.43\left(\mathrm{~m}, 3 \mathrm{H}, \mathrm{H}_{25}+\mathrm{H}_{63}+\mathrm{H}_{98}\right), 9.04-8.98\left(\mathrm{~m}, 2 \mathrm{H}, \mathrm{H}_{88}\right.$ $\left.+\mathrm{H}_{53}\right), 8.53\left(\mathrm{~s}, 1 \mathrm{H}, \mathrm{H}_{109}\right), 8.21-8.16\left(\mathrm{~m}, 1 \mathrm{H}, \mathrm{H}_{22}\right), 8.13-8.05\left(\mathrm{~m}, 2 \mathrm{H}, \mathrm{H}_{60}+\mathrm{H}_{95}\right), 8.05-8.00$ $\left(\mathrm{m}, 4 \mathrm{H}, \mathrm{H}_{56}+\mathrm{H}_{91}\right), 8.00-7.95\left(\mathrm{~m}, 4 \mathrm{H}, \mathrm{H}_{57}+\mathrm{H}_{92}\right), 7.89-7.80\left(\mathrm{~m}, 7 \mathrm{H}, \mathrm{H}_{144}+\mathrm{H}_{153}+\mathrm{H}_{15}+\mathrm{H}_{34}\right.$ $\left.+\mathrm{H}_{72}\right), 7.77\left(\mathrm{~d}, J=8.0 \mathrm{~Hz}, 2 \mathrm{H}, \mathrm{H}_{19}\right), 7.69\left(\mathrm{~d}, J=7.1 \mathrm{~Hz}, 1 \mathrm{H}, \mathrm{H}_{164}\right), 7.64-7.61\left(\mathrm{~m}, 2 \mathrm{H}, \mathrm{H}_{18}\right)$, $7.61-7.56\left(\mathrm{~m}, 6 \mathrm{H}, \mathrm{H}_{27}+\mathrm{H}_{65}+\mathrm{H}_{100}\right) 7.56-7.53\left(\mathrm{~m}, 2 \mathrm{H}, \mathrm{H}_{107}\right), 7.51\left(\mathrm{t}, J=7.8 \mathrm{~Hz}, 1 \mathrm{H}, \mathrm{H}_{125}\right)$, $7.49-7.46\left(\mathrm{~m}, 4 \mathrm{H}, \mathrm{H}_{71}+\mathrm{H}_{33}\right), 7.45-7.39\left(\mathrm{~m}, 4 \mathrm{H}, \mathrm{H}_{76}+\mathrm{H}_{115}\right), 7.39-7.37\left(\mathrm{~m}, 2 \mathrm{H}, \mathrm{H}_{38}\right), 7.37$ - $7.33\left(\mathrm{~m}, 6 \mathrm{H}, \mathrm{H}_{150}\right), 7.32-7.26\left(\mathrm{~m}, 6 \mathrm{H}, \mathrm{H}_{4}\right), 7.25-7.19\left(\mathrm{~m}, 13 \mathrm{H}, \mathrm{H}_{49}+\mathrm{H}_{161}+\mathrm{H}_{162}+\mathrm{H}_{163}+\right.$ $\left.\mathrm{H}_{151}\right), 7.18-7.14\left(\mathrm{~m}, 7 \mathrm{H}, \mathrm{H}_{152}+\mathrm{H}_{106}+\mathrm{H}_{28}\right), 7.14-7.11\left(\mathrm{~m}, 10 \mathrm{H}, \mathrm{H}_{5}+\mathrm{H}_{66}+\mathrm{H}_{101}\right), 7.09-7.05$ (m, 2H, $\left.\mathrm{H}_{77} / \mathrm{H}_{116}\right), 7.05-7.02\left(\mathrm{~m}, 2 \mathrm{H}, \mathrm{H}_{77} / \mathrm{H}_{116}\right), 7.01$ (d, J = $\left.7.7 \mathrm{~Hz}, 2 \mathrm{H}, \mathrm{H}_{126}\right), 7.00-6.97(\mathrm{~m}$, $\left.3 \mathrm{H}, \mathrm{H}_{9}+\mathrm{H}_{139}\right), 6.97-6.92\left(\mathrm{~m}, 4 \mathrm{H}, \mathrm{H}_{39}+\mathrm{H}_{141}\right), 6.87-6.82\left(\mathrm{~m}, 2 \mathrm{H}, \mathrm{H}_{50}\right), 6.80-6.75(\mathrm{~m}, 4 \mathrm{H}$, $\left.\mathrm{H}_{132}\right), 6.52\left(\mathrm{~d}, J=8.8 \mathrm{~Hz}, 2 \mathrm{H}, \mathrm{H}_{10}\right), 6.44\left(\mathrm{~d}, J=8.6 \mathrm{~Hz}, 1 \mathrm{H}, \mathrm{H}_{81}\right), 6.42\left(\mathrm{~d}, J=8.5 \mathrm{~Hz}, 2 \mathrm{H}, \mathrm{H}_{133}\right)$, $6.40\left(\mathrm{~d}, J=8.5 \mathrm{~Hz}, 2 \mathrm{H}, \mathrm{H}_{133^{\prime}}\right), 6.37-6.32\left(\mathrm{~m}, 2 \mathrm{H}, \mathrm{H}_{167}+\mathrm{H}_{43}\right), 5.11-5.00\left(\mathrm{~m}, 5 \mathrm{H}, \mathrm{H}_{23}+\mathrm{H}_{61}+\right.$ $\left.\mathrm{H}_{96}+\mathrm{H}_{159}\right), 4.99-4.90\left(\mathrm{~m}, 1 \mathrm{H}, \mathrm{H}_{119}\right), 4.63-4.57\left(\mathrm{~m}, 2 \mathrm{H}, \mathrm{H}_{124}+\mathrm{H}_{146}\right), 4.57-4.50\left(\mathrm{~m}, 2 \mathrm{H}, \mathrm{H}_{42}\right.$ $\left.+\mathrm{H}_{155}\right), 4.48-4.43\left(\mathrm{~m}, 1 \mathrm{H}, \mathrm{H}_{124^{\prime}}\right), 4.43-4.38\left(\mathrm{~m}, 1 \mathrm{H}, \mathrm{H}_{143}\right), 4.35\left(\mathrm{td}, J=8.7,5.2 \mathrm{~Hz}, 1 \mathrm{H}, \mathrm{H}_{80}\right)$, $4.24\left(\mathrm{dd}, J=15.3,5.6 \mathrm{~Hz}, 1 \mathrm{H}, \mathrm{H}_{143^{\circ}}\right), 4.02\left(\mathrm{t}, J=7.1 \mathrm{~Hz}, 2 \mathrm{H}, \mathrm{H}_{14}\right), 3.73\left(\mathrm{~s}, 3 \mathrm{H}, \mathrm{H}_{52}\right), 3.72-$ $3.65\left(\mathrm{~m}, 6 \mathrm{H}, \mathrm{H}_{166}+\mathrm{H}_{135}\right), 3.61-3.54\left(\mathrm{~m}, 1 \mathrm{H}, \mathrm{H}_{123}\right), 3.54-3.46\left(\mathrm{~m}, 1 \mathrm{H}, \mathrm{H}_{123}\right), 3.43(\mathrm{t}, J=6.0$ $\left.\mathrm{Hz}, 2 \mathrm{H}, \mathrm{H}_{12}\right), 3.40-3.34\left(\mathrm{~m}, 2 \mathrm{H}, \mathrm{H}_{74} / \mathrm{H}_{113}\right), 3.31\left(\mathrm{dd}, J=13.9,6.2 \mathrm{~Hz}, 1 \mathrm{H}, \mathrm{H}_{36}\right), 3.26-3.21$ $\left(\mathrm{m}, 2 \mathrm{H}, \mathrm{H}_{74^{\prime}} / \mathrm{H}_{113^{\prime}}\right) 3.21-3.15\left(\mathrm{~m}, 2 \mathrm{H}, \mathrm{H}_{47}+\mathrm{H}_{36^{\prime}}\right), 3.07\left(\mathrm{dd}, J=13.8,8.5 \mathrm{~Hz}, 1 \mathrm{H}, \mathrm{H}_{47^{\prime}}\right), 2.71$ 
$\left(\mathrm{dd}, J=12.4,5.4 \mathrm{~Hz}, 1 \mathrm{H}, \mathrm{H}_{147}\right), 2.67-2.63\left(\mathrm{~m}, 1 \mathrm{H}, \mathrm{H}_{147^{\circ}}\right), 2.63-2.59\left(\mathrm{~m}, 4 \mathrm{H}, \mathrm{H}_{128}\right), 2.55-$ $2.46\left(\mathrm{~m}, 10 \mathrm{H}, \mathrm{H}_{157}+\mathrm{H}_{138}+\mathrm{H}_{130}\right), 2.21-2.15\left(\mathrm{~m}, 1 \mathrm{H}, \mathrm{H}_{156}\right), 2.03-1.98\left(\mathrm{~m}, 1 \mathrm{H}, \mathrm{H}_{156}\right), 1.89-$ $1.81\left(\mathrm{~m}, 7 \mathrm{H}, \mathrm{H}_{86}+\mathrm{H}_{13}+\mathrm{H}_{129}\right), 1.77-1.68\left(\mathrm{~m}, 12 \mathrm{H}, \mathrm{H}_{85}+\mathrm{H}_{69} / \mathrm{H}_{104}+\mathrm{H}_{137}\right), 1.68-1.57(\mathrm{~m}, 16 \mathrm{H}$, $\left.\mathrm{H}_{69} / \mathrm{H}_{104}+\mathrm{H}_{31}+\mathrm{H}_{136}\right), 1.46-1.40\left(\mathrm{~m}, 9 \mathrm{H}, \mathrm{H}_{122}\right), 1.40-1.38\left(\mathrm{~m}, 9 \mathrm{H}, \mathrm{H}_{84}\right), 1.37-1.36(\mathrm{~m}, 9 \mathrm{H}$, $\left.\mathrm{H}_{46} / \mathrm{H}_{170}\right), 1.36-1.34\left(\mathrm{~m}, 9 \mathrm{H}, \mathrm{H}_{46} / \mathrm{H}_{170}\right), 1.28\left(\mathrm{~s}, 27 \mathrm{H}, \mathrm{H}_{1}\right), 1.26\left(\mathrm{~s}, 9 \mathrm{H}, \mathrm{H}_{112}\right), 0.99-0.93(\mathrm{~m}$, $\left.6 \mathrm{H}, \mathrm{H}_{87}\right) ;{ }^{13} \mathrm{C}$ NMR $\left(151 \mathrm{MHz}\right.$, Acetone- $\left.d_{6}\right) \delta 176.06,172.44,171.83,171.06,170.80,170.27$, $169.81,169.70,169.58,166.56,166.29,161.60,158.75,157.15,157.13,156.42,156.29$, $155.78,155.46,151.71,151.68,149.77,149.63,148.20,146.89,146.23,145.94,145.52$, $145.33,144.72$, 144.44, 142.70, 139.31, 138.73, 137.25, 136.80, 136.73, 136.67, 136.48, $136.45,135.50,134.90,134.19,133.83,133.63,133.40,132.60,131.81,131.75,130.55$, $130.36,129.44,129.12,128.87,128.39,128.14,128.10,128.00,127.95,127.91,127.89$, $127.59,127.02,126.98,126.92,126.80,126.73,126.67,125.25,125.08,124.89,124.18$, $121.37,121.26,121.17,119.83,119.71,119.56,119.54,119.47,119.44,119.28,114.10$, $114.08,113.79,113.28,80.40,78.85,78.67,78.51,68.81,67.23,67.21,66.48,65.74,63.85$, $63.01,61.75,61.55,56.02,55.91,55.78,54.59,52.98,52.54,48.79,46.60,44.11,42.78$, $42.35,41.85,40.13,39.23,37.55,37.03,36.96,36.39,35.32,35.29,35.24,34.73,34.25$, $33.98,33.29,32.86,32.11,31.75,31.10,30.80,30.25,30.06,29.98,29.70,28.25,27.77$, 27.66, 27.54, 27.21, 26.88, 24.78, 24.68, 22.44, 22.37, 20.89, 13.47; HRMS (ESI $\left.{ }^{+}\right)$Calculated for $\mathrm{C}_{258} \mathrm{H}_{287} \mathrm{O}_{30} \mathrm{~N}_{24} \mathrm{~S}_{2} \mathrm{Na}[\mathrm{M}+\mathrm{Na}+\mathrm{H}]^{2+} 2145.5539$, found 2145.5532 . 


\subsubsection{Synthesis of machine 2}

2

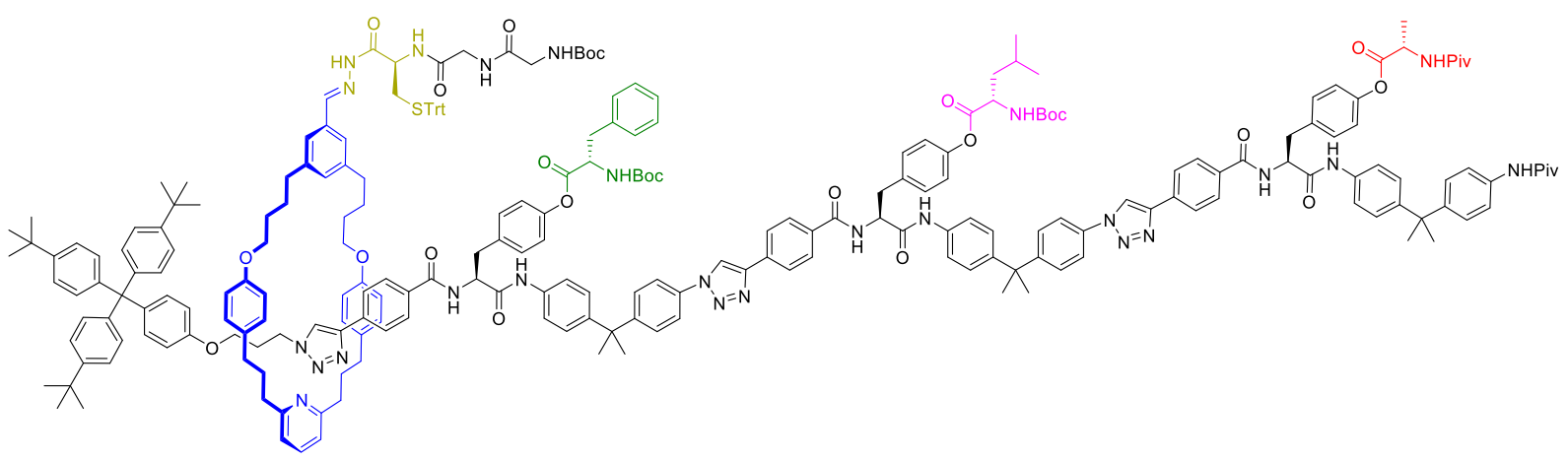

Tentagel $^{\mathrm{TM}}$ TBTA resin (294 mg, 0.05 mmol, 0.5 equiv.) and $\mathrm{Cu}(\mathrm{MeCN})_{4} \mathrm{PF}_{6}$ (17 mg, 0.045 mmol, 0.45 equiv.) were added to a sealed microwave vial. The vial was purged by repeated cycles of vacuum evacuation/filling with argon. The mixture was suspended in $\mathrm{CH}_{2} \mathrm{Cl}_{2}(10 \mathrm{~mL})$ which was sparged with argon prior to use. The mixture was stirred for 10 minutes. To the mixture was added a solution of 4 (190 mg, $98 \mu \mathrm{mol}, 1.0$ equiv.) and 6 (148 mg, $98 \mu \mathrm{mol}, 1.0$ equiv.) in a degassed mixture of $\mathrm{CH}_{2} \mathrm{Cl}_{2}(10 \mathrm{~mL})$ and $t \mathrm{BuOH}(2.5 \mathrm{~mL})$. The reaction was stirred for $48 \mathrm{~h}$ at room temperature. The reaction was filtered (to recover and recycle TBTA resin) and the solvent was removed under reduced pressure. The crude residue was purified by size exclusion chromatography ( $\mathrm{SX}-1$ beads, $\mathrm{CH}_{2} \mathrm{Cl}_{2}$ ) to afford the corresponding rotaxane as a yellow solid (290 mg, $84 \mu \mathrm{mol}, 86 \%)$.

To a solution of rotaxane (140 mg, $40.5 \mu \mathrm{mol}, 1.0$ equiv.) and Boc-Gly-Gly-Cys(Trt)-NHNH (26 mg, $44.6 \mu \mathrm{mol}, 1.1$ equiv.) in $\mathrm{CH}_{2} \mathrm{Cl}_{2}(4 \mathrm{~mL}$ ) was added aniline (7 drops). The mixture was stirred at room temperature for $48 \mathrm{~h}$. The crude mixture was purified by flash column chromatography $\left(\mathrm{CH}_{2} \mathrm{Cl}_{2} / \mathrm{MeOH} 30: 1\right)$ to deliver 2 as a yellow glassy solid (81 mg, $20.1 \mu \mathrm{mol}$, $50 \%$ ). Data consistent with that previously reported. ${ }^{1}$ 


\subsection{Operation of molecular machine 1}

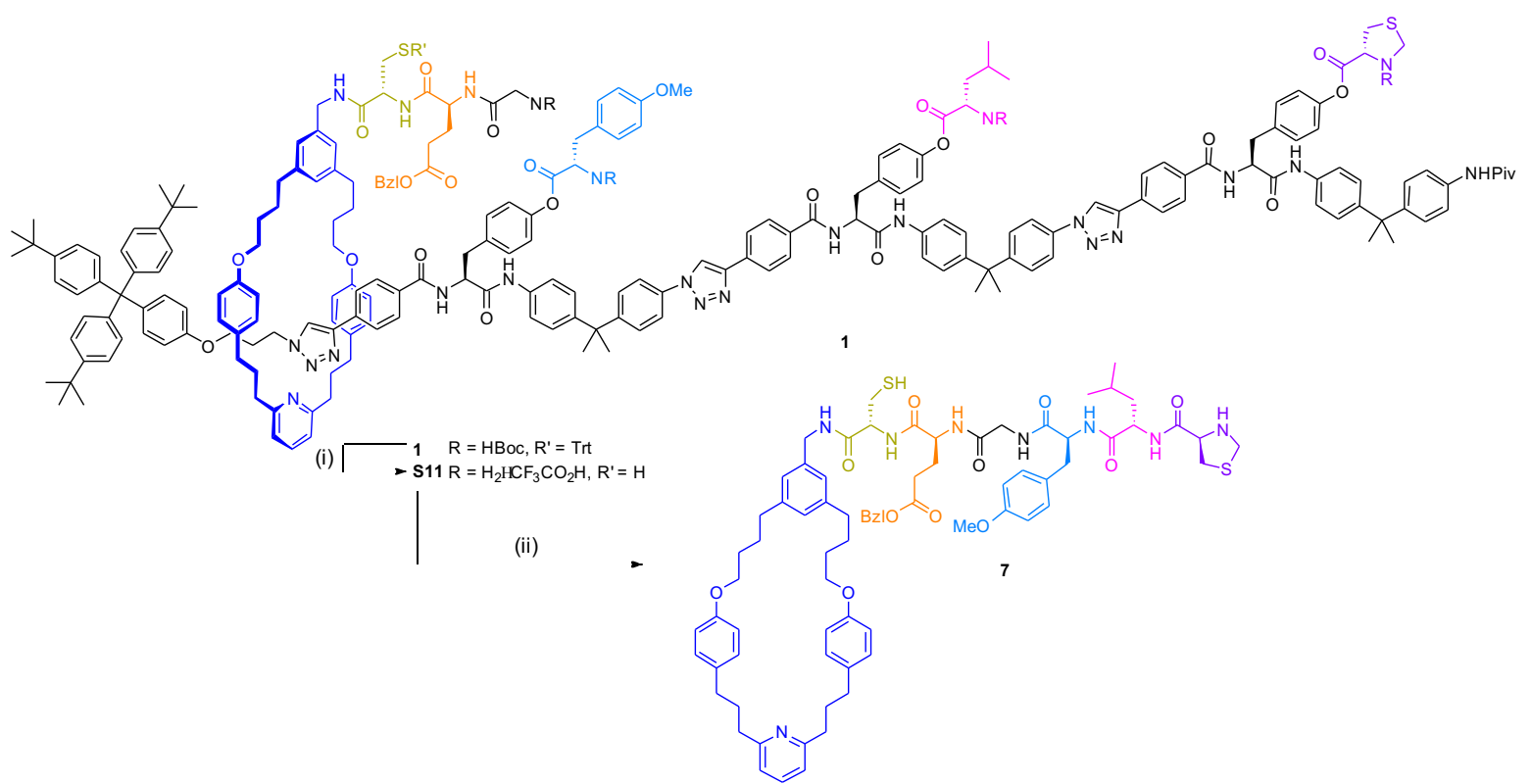

Scheme S8. Operation of molecular machine 1. Reagents and conditions: (i) $\mathrm{TIPSH}, \mathrm{CH}_{2} \mathrm{Cl}_{2} / \mathrm{CF}_{3} \mathrm{CO}_{2} \mathrm{H}$ (4:1) rt, 0.5 h. (ii) $i-\operatorname{Pr}_{2} \mathrm{EtN}, 4 \AA$ molecular sieves, MeCN/DMF (3:1), MW, $75{ }^{\circ} \mathrm{C}, 15 \mathrm{~h}$.

Molecular machine 1 (1 mg, $0.25 \mu$ mol, 1 equiv.) was dissolved in anhydrous $\mathrm{CH}_{2} \mathrm{Cl}_{2}(0.4 \mathrm{~mL})$, $\mathrm{CF}_{3} \mathrm{CO}_{2} \mathrm{H}(0.1 \mathrm{~mL})$ was added followed immediately by TIPSH (1 drop) after which the yellow solution became colourless in ca. $5 \mathrm{~min}$. The resulting solution was stirred for $0.5 \mathrm{~h}$ at room temperature. Toluene $(0.5 \mathrm{~mL})$ was added and the solution concentrated to dryness under reduced pressure. The resulting solid was further azeotroped with toluene $(2 \times 0.5 \mathrm{~mL})$ under reduced pressure. The resulting solid was triturated with $\mathrm{Et}_{2} \mathrm{O}: n$-hexane $(1: 1,0.5 \mathrm{~mL})$. The remaining solid was dried thoroughly under reduced pressure to give the crude deprotected machine S11. The crude deprotected machine $\mathbf{S 1 1}$ (assumed $0.25 \mu \mathrm{mol}, 1$ equiv.) was dissolved in degassed MeCN:DMF (3:1, $2 \mathrm{~mL}, 0.125 \mathrm{mM})$. DIPEA $(0.12 \mu \mathrm{L}, 0.75 \mu \mathrm{mol}, 3.0$ equiv.) and $4 \AA$ molecular sieves were added and the resulting solution was stirred under microwave heating at $75{ }^{\circ} \mathrm{C}$ for $15 \mathrm{~h}$ under argon. The operation mixture was cooled to room temperature and the solvent was removed under reduced pressure. The crude operation mixture was analysed by LRMS indicating the formation of 7 (see figure S3). 
SI Echavarren et al, 'Sequence-Selective Decapeptide Synthesis by the Parallel Operation of ...'

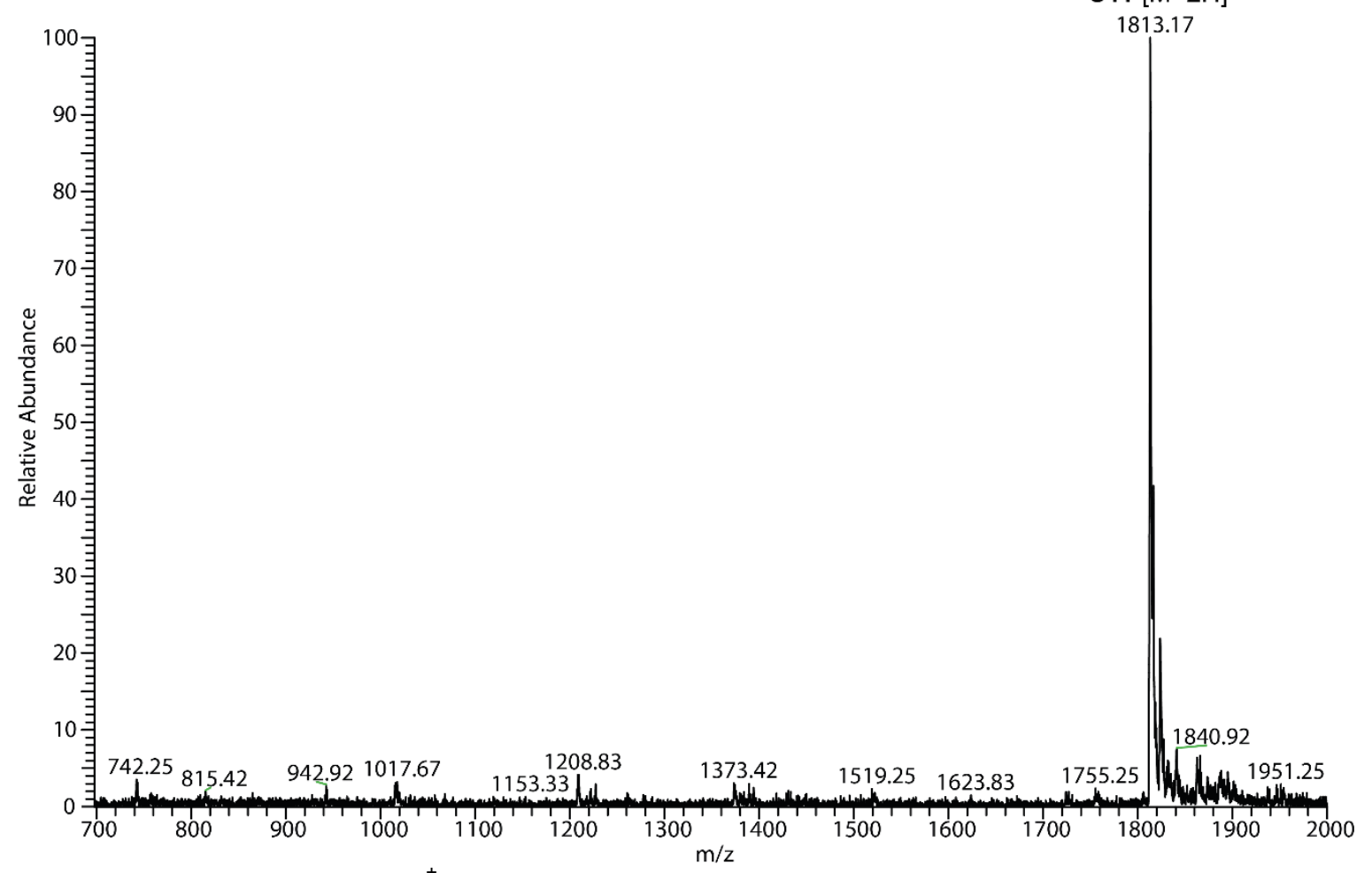

Figure S2. Mass spectrum $\left(\mathrm{ESI}^{+}\right)$of crude deprotected machine S11.

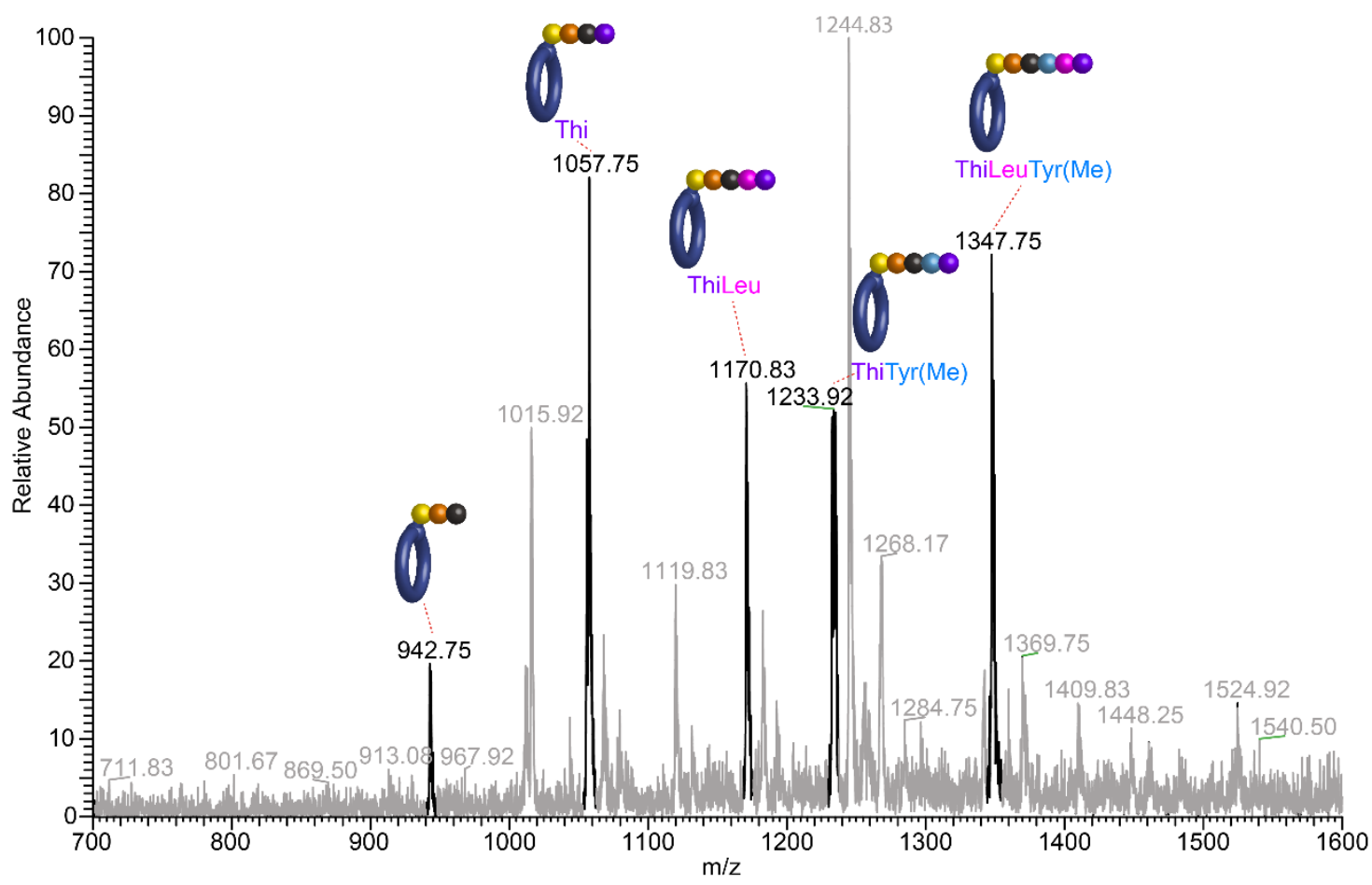

Figure S3. Mass spectrum $\left(\mathrm{ESI}^{+}\right)$of crude operation mixture of 1. 
SI Echavarren et al, 'Sequence-Selective Decapeptide Synthesis by the Parallel Operation of ...'
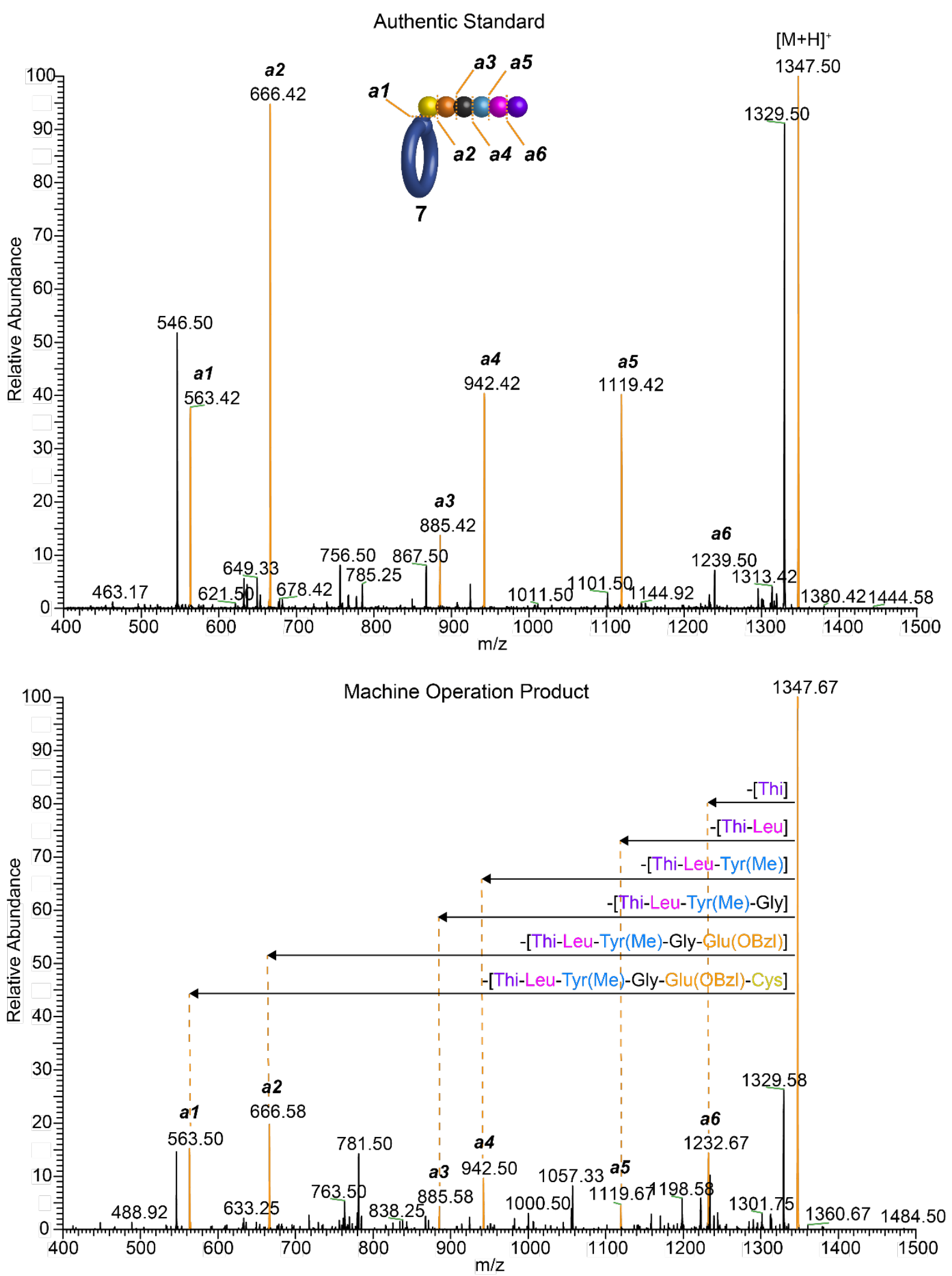

Figure S4. Tandem mass spectra of $[7+\mathrm{H}]^{+}$ions of peptide appended macrocycle bearing the sequence ThiLeuTyr(Me)GlyGlu(OBzl)Cys. Top: MS-MS spectrum of authentic sample prepared by conventional means. Bottom: MS-MS spectrum of the product of operation of molecular machine 1. Orange peaks indicate amide bond $\mathrm{C}-\mathrm{N}$ fragmentation. 


\subsection{Operation of molecular machine 2}

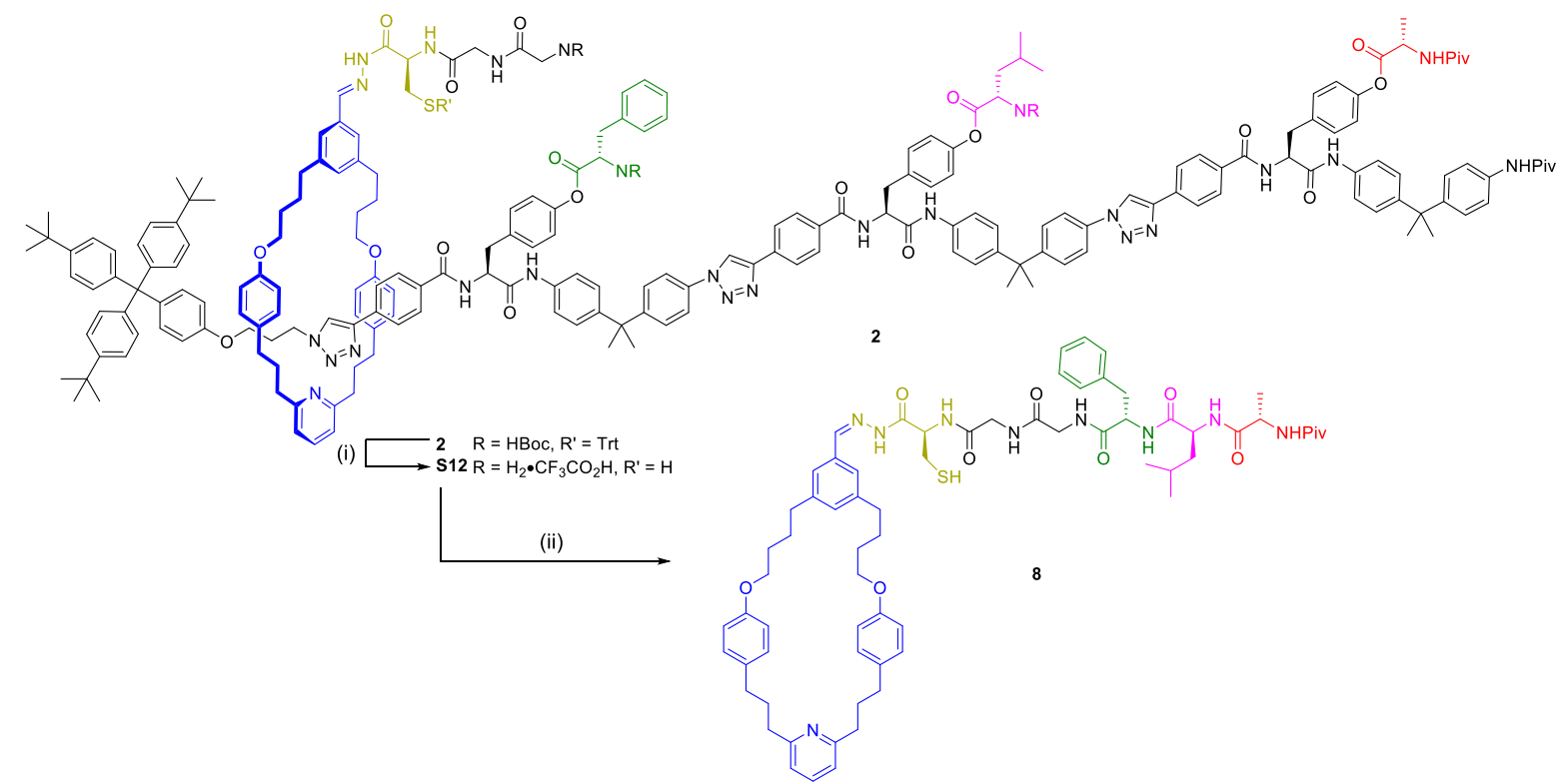

Scheme S9. Operation of molecular machine 2. Reagents and conditions: (i) TIPSH, $\mathrm{CH}_{2} \mathrm{Cl}_{2} / \mathrm{CF}_{3} \mathrm{CO}_{2} \mathrm{H}$ (4:1) rt, 0.5 h. (ii) $i-\mathrm{Pr}_{2} \mathrm{EtN}, 4 \AA$ molecular sieves, MeCN/DMF (3:1), MW, $75^{\circ} \mathrm{C}, 15 \mathrm{~h}$.

Molecular machine 2 ( $1 \mathrm{mg}, 0.25 \mu$ mol, 1 equiv.) was dissolved in anhydrous $\mathrm{CH}_{2} \mathrm{Cl}_{2}(0.4 \mathrm{~mL})$, $\mathrm{CF}_{3} \mathrm{CO}_{2} \mathrm{H}(0.1 \mathrm{~mL})$ was added followed immediately by TIPSH (1 drop) after which the yellow solution became colourless in ca. $5 \mathrm{~min}$. The resulting solution was stirred for $0.5 \mathrm{~h}$ at room temperature. Toluene $(0.5 \mathrm{~mL})$ was added and the solution concentrated to dryness under reduced pressure. The resulting solid was further azeotroped with toluene $(2 \times 0.5 \mathrm{~mL})$ under reduced pressure. The resulting solid was triturated with $\mathrm{Et}_{2} \mathrm{O}: n$-hexane $(1: 1,0.5 \mathrm{~mL})$. The remaining solid was dried thoroughly under reduced pressure to give the crude deprotected machine S12. The crude deprotected machine $\mathbf{S 1 2}$ (assumed $0.25 \mu \mathrm{mol}, 1$ equiv.) was dissolved in degassed MeCN:DMF (3:1, $2 \mathrm{~mL}, 0.125 \mathrm{mM})$. DIPEA $(0.12 \mu \mathrm{L}, 0.75 \mu \mathrm{mol}, 3.0$ equiv.) and $4 \AA$ molecular sieves were added and the resulting solution was stirred under microwave heating at $75^{\circ} \mathrm{C}$ for $15 \mathrm{~h}$ under argon. The operation mixture was cooled to room temperature and the solvent was removed under reduced pressure. The crude operation mixture was analysed by LRMS indicating the formation of 8 (see figure S6). 
SI Echavarren et al, 'Sequence-Selective Decapeptide Synthesis by the Parallel Operation of ...'

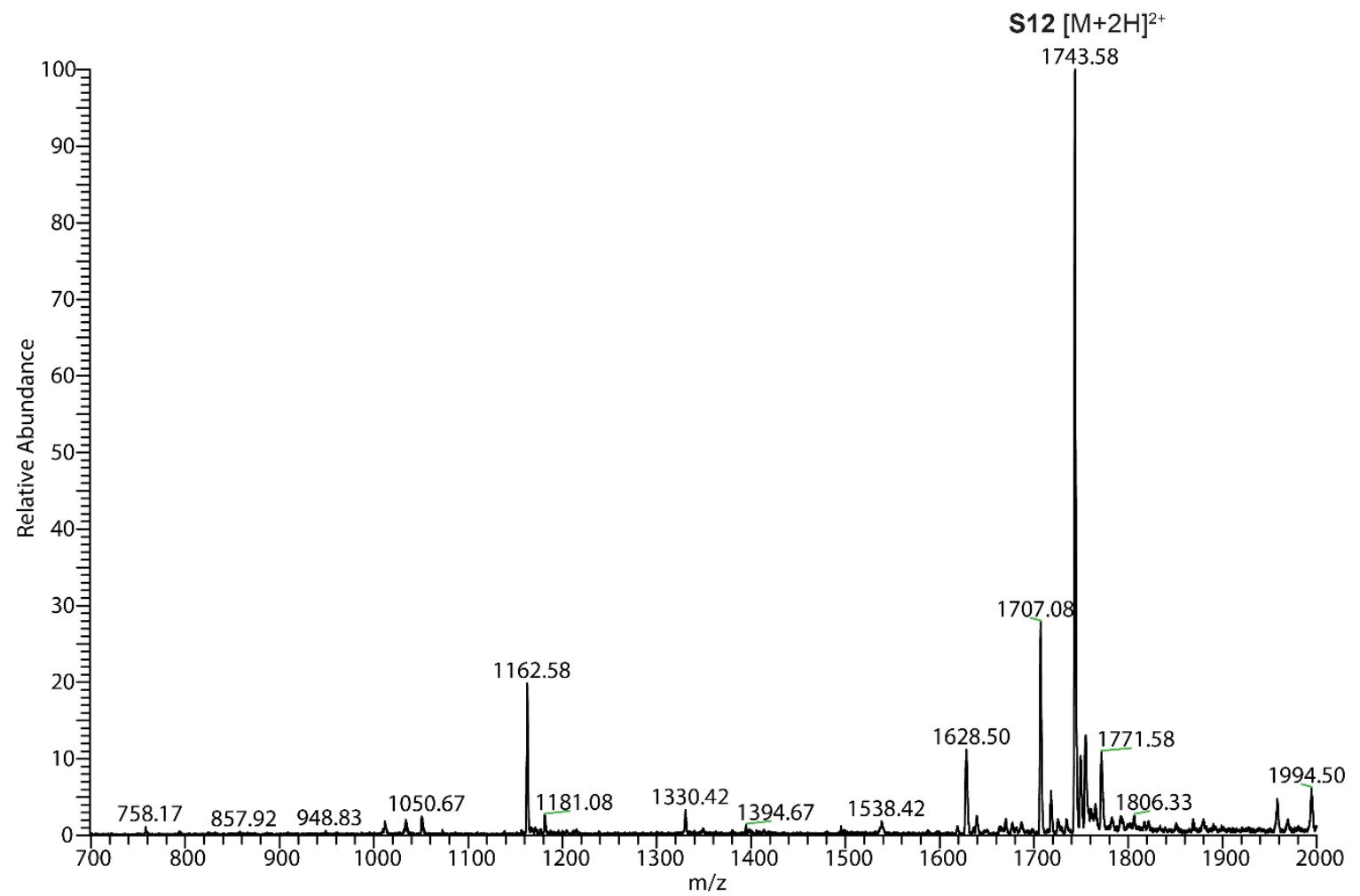

Figure S5. Mass spectrum $\left(\mathrm{ESI}^{+}\right)$of crude deprotected machine S12.

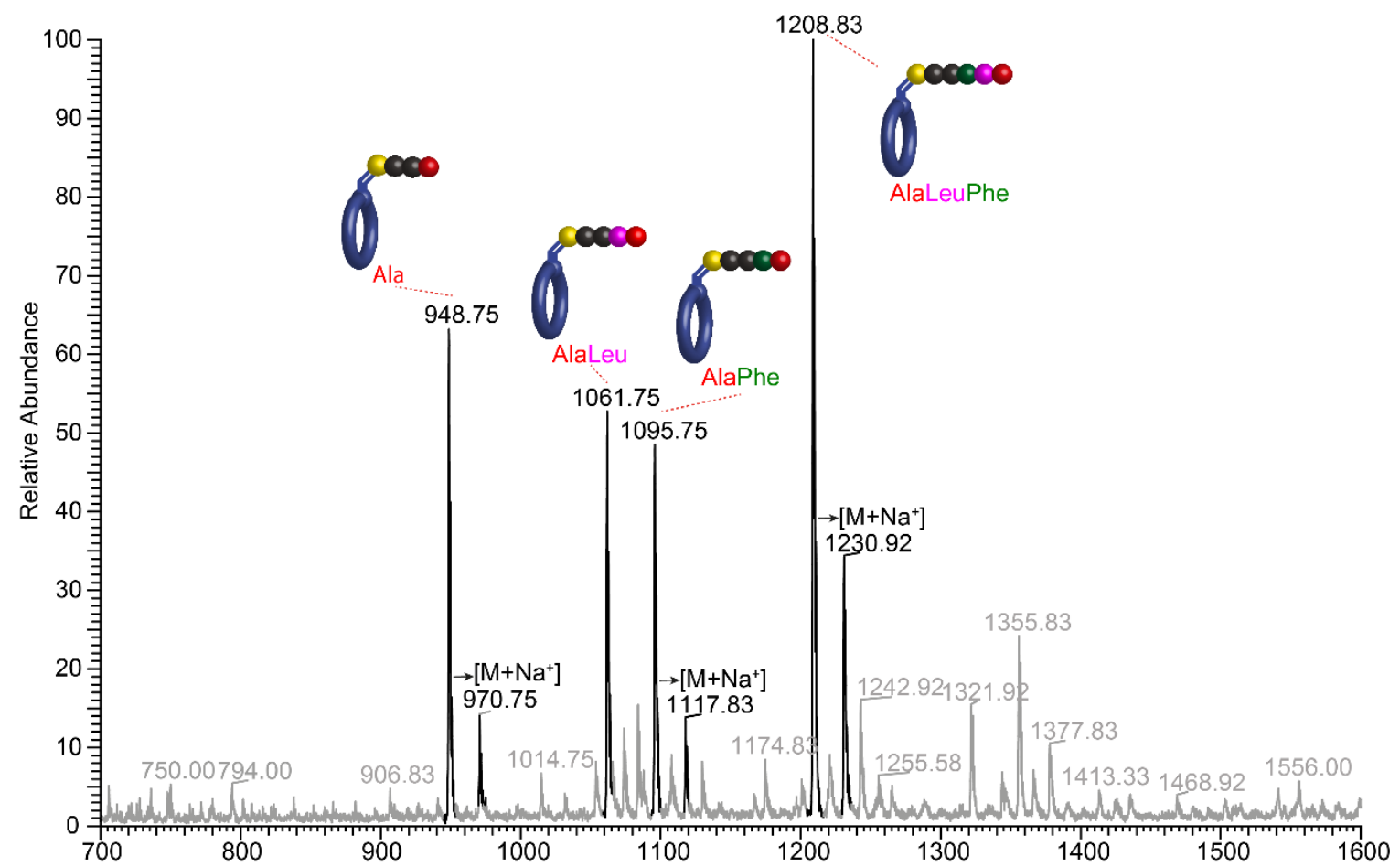

Figure S6. Mass spectrum $\left(\mathrm{ESI}^{+}\right)$of crude operation mixture of 2. 
SI Echavarren et al, 'Sequence-Selective Decapeptide Synthesis by the Parallel Operation of ...'
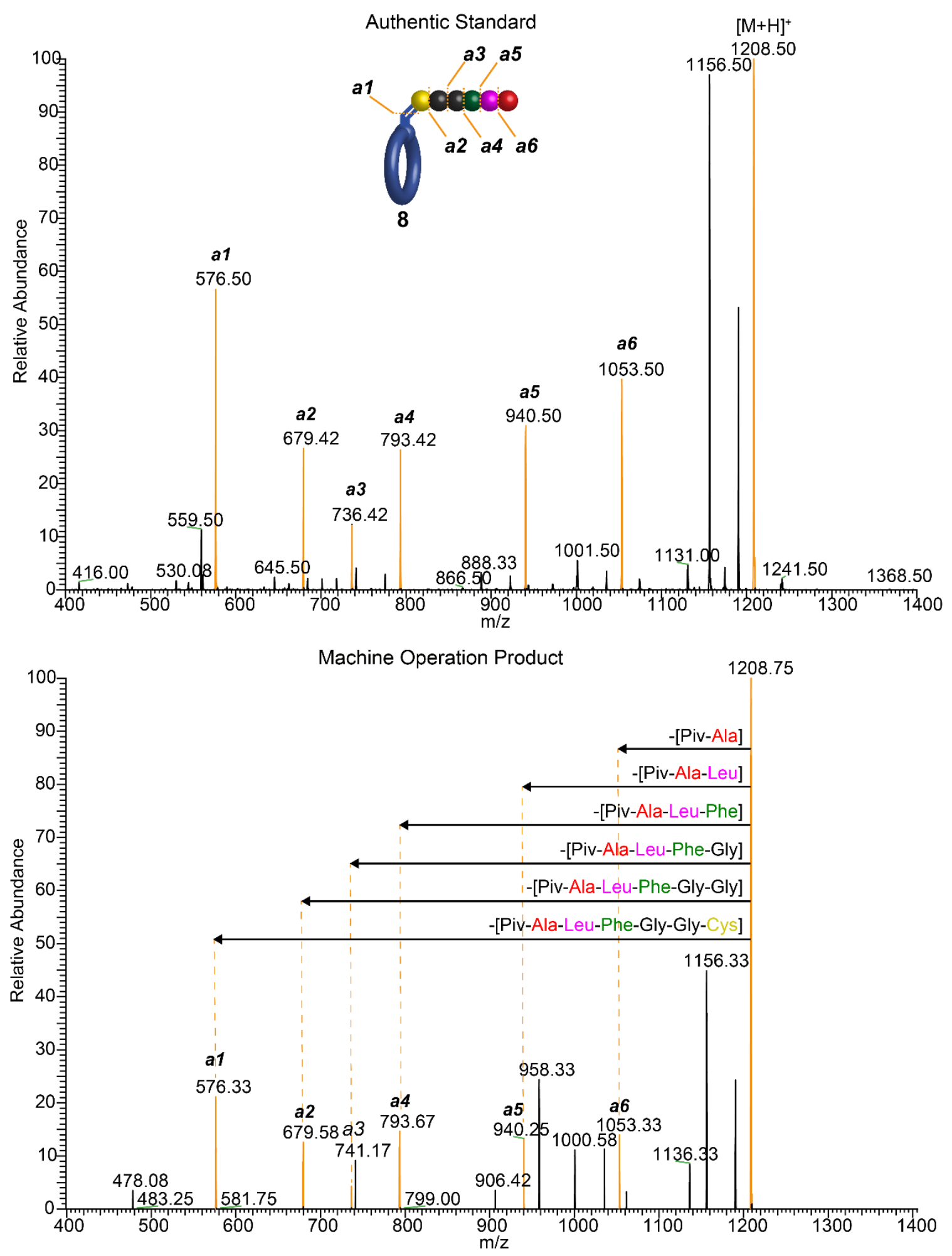

Figure S7. Tandem mass spectra of $[\mathbf{8}+\mathrm{H}]+$ ions of peptide appended macrocycle bearing the sequence PivAlaLeuPheGlyGlyCys. Top: MS-MS spectrum of authentic sample prepared by conventional means. Bottom: MS-MS spectrum of the product of operation of molecular machine 2. Orange peaks indicate amide bond $\mathrm{C}-\mathrm{N}$ fragmentation. 


\subsection{Operation of molecular machines 1 and 2 in one pot}

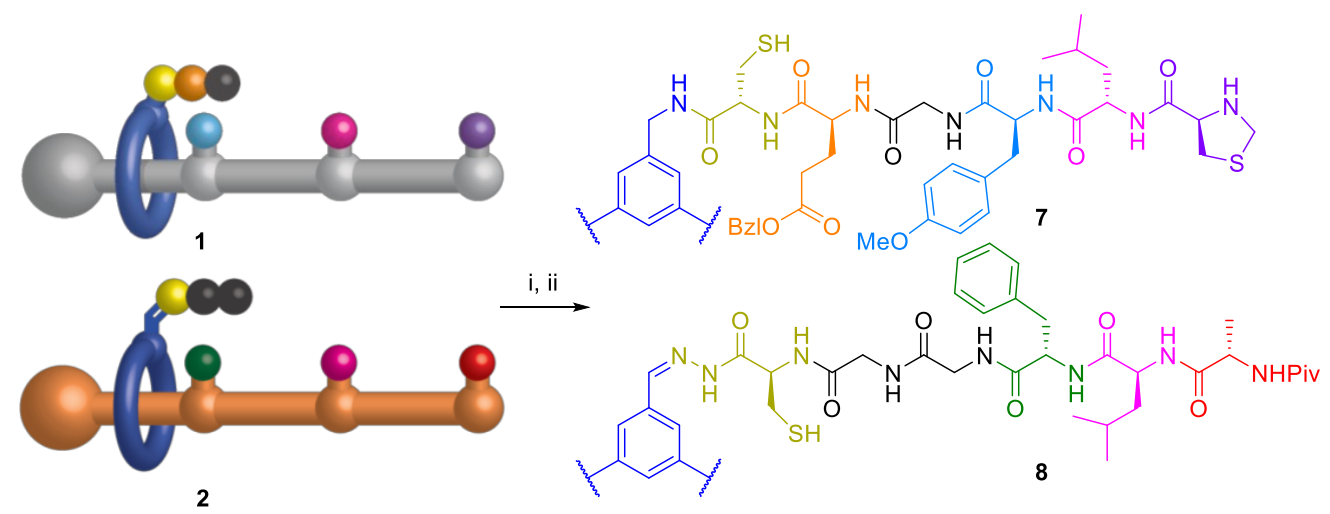

Scheme S8. Operation of molecular machines 1 and $\mathbf{2}$ in one pot. Reagents and conditions: (i) TIPSH, $\mathrm{CH}_{2} \mathrm{Cl}_{2} / \mathrm{CF}_{3} \mathrm{CO}_{2} \mathrm{H}$ (4:1), rt, $0.5 \mathrm{~h}$. (ii) $i-\mathrm{Pr}_{2} \mathrm{EtN}, 4 \AA$ molecular sieves, MeCN/DMF (3:1), MW, $75^{\circ} \mathrm{C}, 15 \mathrm{~h}$.

Molecular machine 1 ( $0.5 \mathrm{mg}, 0.125 \mu \mathrm{mol}, 1$ equiv) and 2 ( $1 \mathrm{mg}, 0.25 \mu \mathrm{mol}, 2$ equiv.) was dissolved in anhydrous $\mathrm{CH}_{2} \mathrm{Cl}_{2}(0.6 \mathrm{~mL}), \mathrm{CF}_{3} \mathrm{CO}_{2} \mathrm{H}(0.15 \mathrm{~mL})$ was added followed immediately by triisopropylsilane ( 2 drops) after which the yellow solution became colourless (ca. $5 \mathrm{~min}$ ). The resulting solution was stirred for $0.5 \mathrm{~h}$ at room temperature. Toluene $(0.75 \mathrm{~mL})$ was added and the solution concentrated to dryness under reduced pressure. The resulting solid was further azeotroped with toluene $(2 \times 0.75 \mathrm{~mL})$ under reduced pressure. The resulting solid was triturated with $\mathrm{Et}_{2} \mathrm{O}: n$-hexane $(1: 1,0.75 \mathrm{~mL})$. The remaining solid was dried thoroughly under reduced pressure to give the crude deprotected machines $\mathbf{S 1 1}$ and $\mathbf{S 1 2}$. The crude deprotected machines S11 (assumed $0.125 \mu \mathrm{mol}, 1$ equiv.) and S12 (assumed $0.250 \mu \mathrm{mol}, 2$ equiv.) was dissolved in degassed MeCN:DMF (3:1, $1.3 \mathrm{~mL}, 0.3 \mathrm{mM})$. DIPEA $(0.18 \mu \mathrm{L}, 1.13$ $\mu \mathrm{mol}, 3.0$ equiv.) and $4 \AA$ molecular sieves were added and the resulting solution was stirred under microwave heating at $75^{\circ} \mathrm{C}$ for $15 \mathrm{~h}$ under argon. The operation mixture was cooled to room temperature and the solvent was removed under reduced pressure. The crude operation mixture was analysed by LRMS indicating the formation of 7 and 8 (see figure S9). 
SI Echavarren et al, 'Sequence-Selective Decapeptide Synthesis by the Parallel Operation of ...'

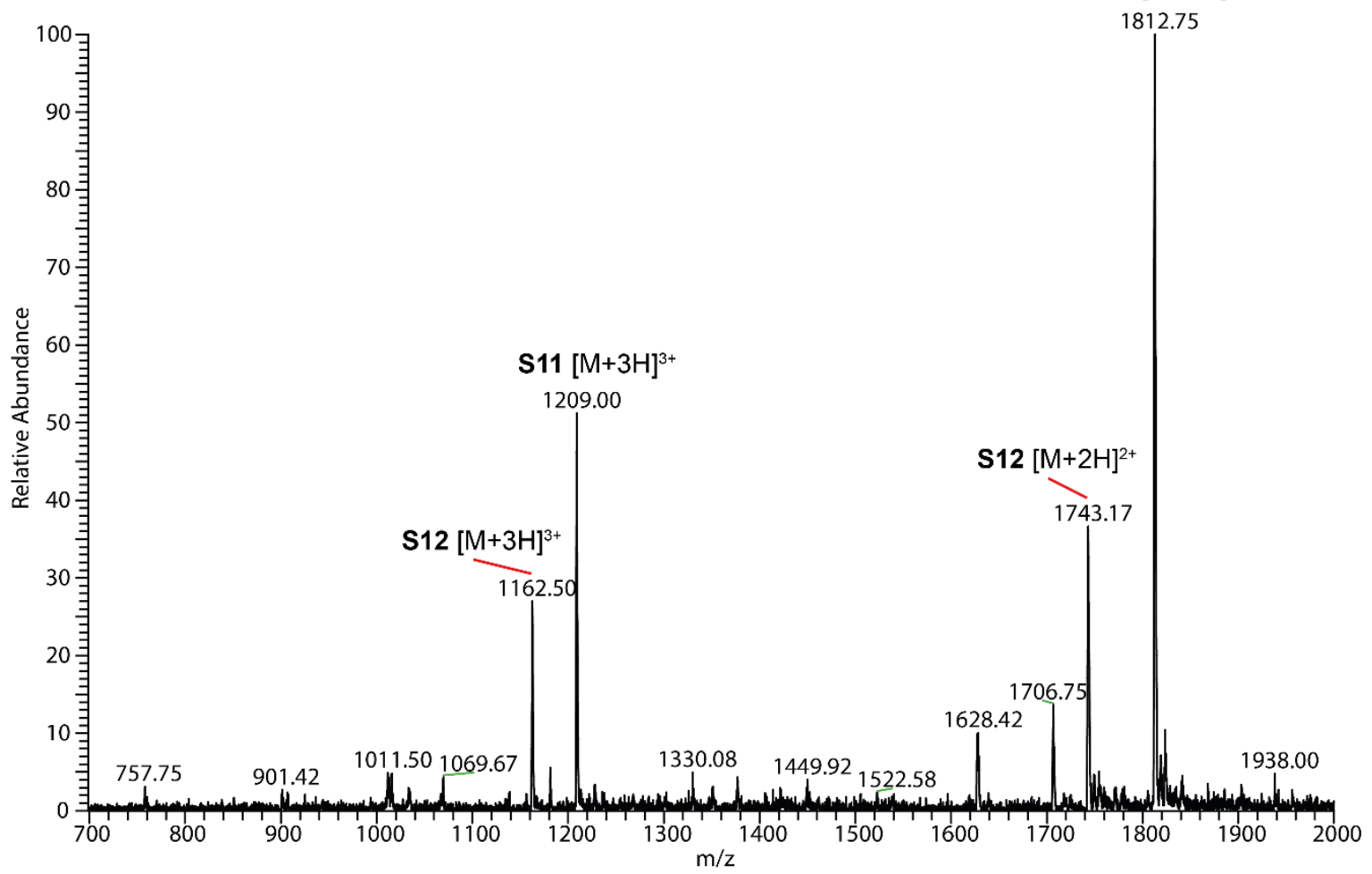

Figure S8. Mass spectrum $\left(\mathrm{ESI}^{+}\right)$of crude deprotected machines S11 and S12.

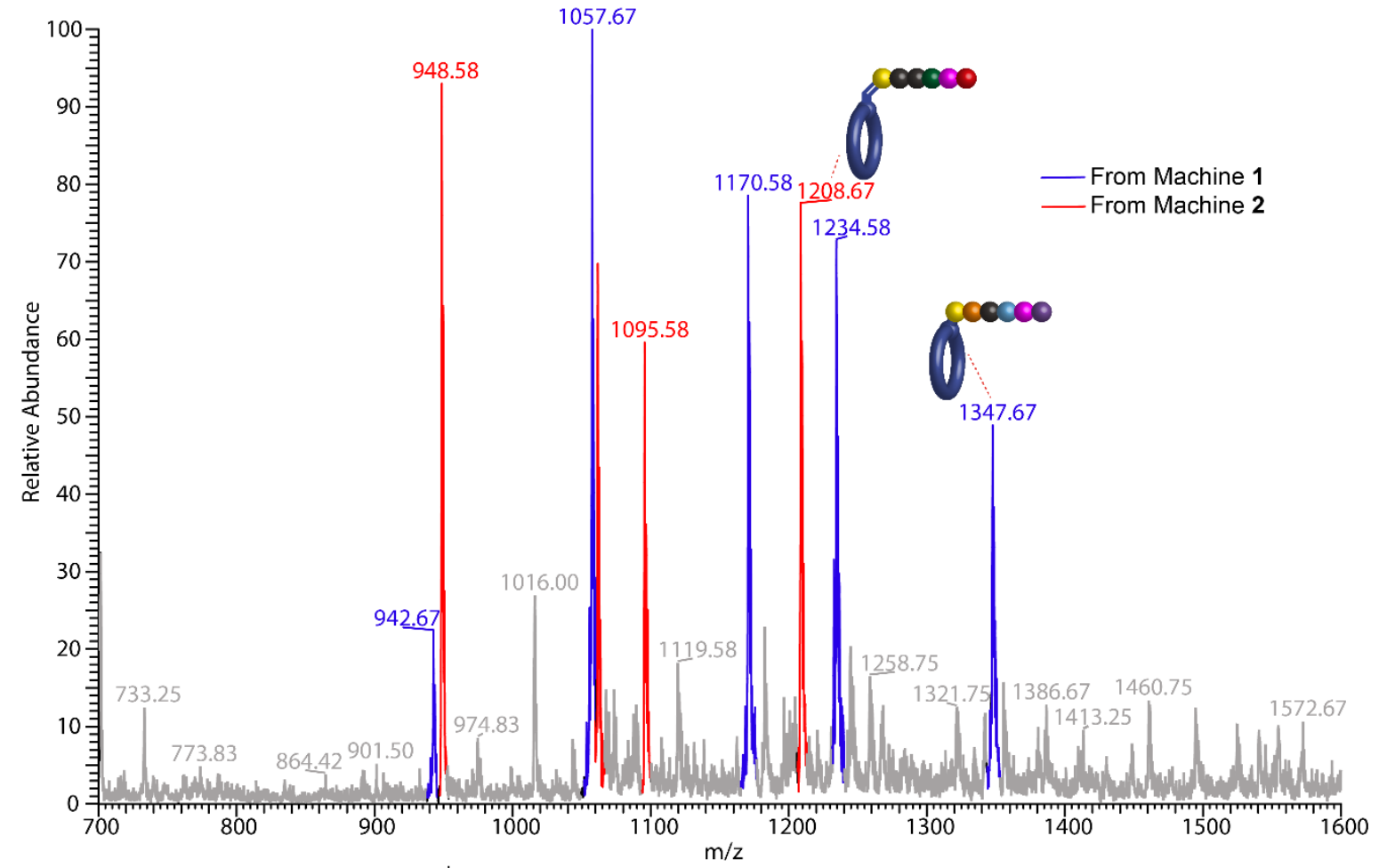

Figure S9. Mass spectrum $\left(\mathrm{ESI}^{+}\right)$of crude operation mixture. 


\subsection{Synthesis of standards of operation}

\subsubsection{General procedures}

General Procedure A: Boc Deprotection in the Presence of Trt.

To a solution of Boc protected peptide (1.0 equiv.) and triphenylmethanol ( 0.5 equiv.) in $\mathrm{CH}_{2} \mathrm{Cl}_{2}$ $(0.05 \mathrm{M})$ was added $\mathrm{CF}_{3} \mathrm{CO}_{2} \mathrm{H}$ acid $(20 \%$ by volume). The reaction mixture was stirred at room temperature for $2 \mathrm{~h}$. The reaction mixture was diluted by $50 \%$ with toluene then the solvent was removed under reduced pressure. The crude residue was azeotroped twice with toluene to remove excess $\mathrm{CF}_{3} \mathrm{CO}_{2} \mathrm{H}$. Trituration with $\mathrm{Et}_{2} \mathrm{O}: n$-hexane $(1: 1$, to remove triphenylmethanol) gave the resulting ammonium salt which was used without further purification.

General Procedure B: Amide Coupling of TFA Salts.

To a solution of the ammonium salt (1.0 equiv.) in $\mathrm{CH}_{2} \mathrm{Cl}_{2}(0.05 \mathrm{M})$ was added $\mathrm{Et}_{3} \mathrm{~N}$ (1.2 equiv.) followed by $\mathrm{EDCl} \cdot \mathrm{HCl}$ (1.2 equiv.), $\mathrm{HOBt} \cdot \mathrm{H}_{2} \mathrm{O}$ (1.2 equiv.) and Boc or Piv protected amino acid (1.2 equiv.). The reaction mixture was stirred at room temperature for $16 \mathrm{~h}$. The mixture was diluted with $\mathrm{CHCl}_{3}$ and washed sequentially with $2 \mathrm{M} \mathrm{NaHCO}_{3}, 1 \mathrm{M} \mathrm{HCl}$ and brine. The organic phase was dried $\left(\mathrm{MgSO}_{4}\right)$ and the solvent was removed under reduced pressure. Purification by flash column chromatography $\left(\mathrm{SiO}_{2}, \mathrm{MeOH} / \mathrm{CH}_{2} \mathrm{Cl}_{2}\right)$ yielded the product. 


\subsubsection{Synthesis of 7 and 9}

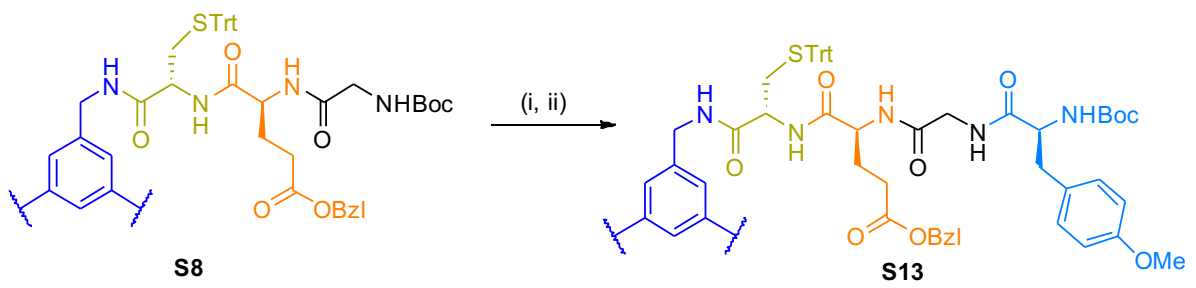

(iii, iv)

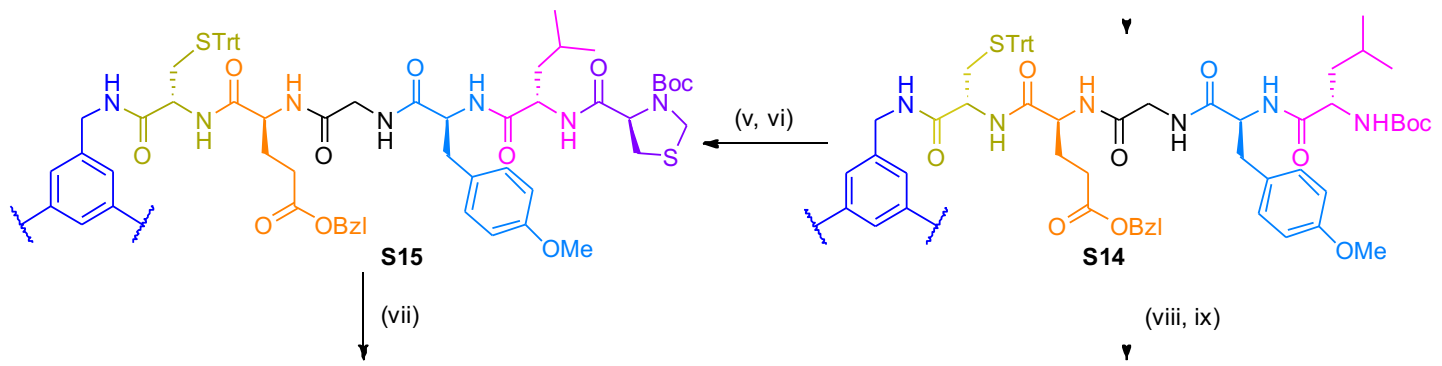<smiles>CCOC(=O)CCC(NC(=O)CNC(=O)C(Cc1ccc(OC)cc1)NC(=O)C(CS)NC(=O)C1CSCN1)C(=O)NC(CC(C)C)C(=O)NCc1cc(C(C)C)cc(C(C)C)c1</smiles>

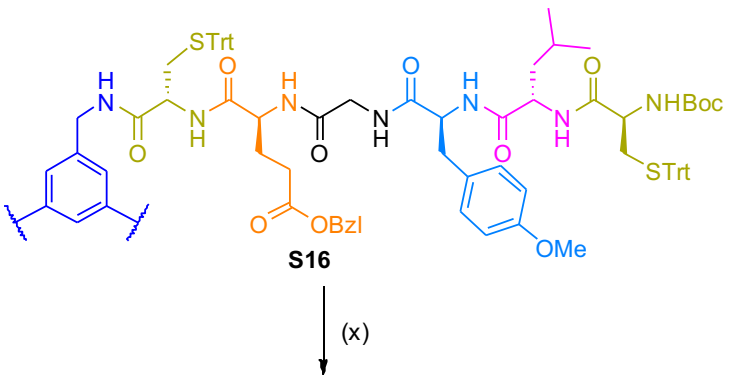<smiles>CCOC(=O)CCC(NC(=O)CNC(=O)C(CSSCC(N)C(=O)N[C@@H](Cc1ccc(OC)cc1)C(=O)NCc1cc(CC)cc(C(C)C)c1)NC(=O)[C@H](C)CC)C(=O)NCc1ccccc1</smiles><smiles>CCOC(=O)CCC(NC(=O)CNC(=O)C(Cc1ccc(OC)cc1)NC(=O)[C@H](CC(C)C)NC(=O)C(N)CS)C(=O)N[C@@H](CS)C(=O)NCc1cc(CC)cc(CC)c1</smiles>

Scheme S11. Synthesis of standards 7 and $\mathbf{9}$. Standard 9 was synthesised via deprotection from intermediate S16. Reagents and conditions: (i) $\mathrm{Ph}_{3} \mathrm{COH}, \mathrm{CF}_{3} \mathrm{CO}_{2} \mathrm{H}, \mathrm{CH}_{2} \mathrm{Cl}_{2}$, rt, 4 h. (ii) Boc- $\mathrm{Tyr}(\mathrm{Me}$ )$\mathrm{OH}, \mathrm{HOBt} \cdot \mathrm{H}_{2} \mathrm{O}$, EDCl$\cdot \mathrm{HCl}, \mathrm{CH}_{2} \mathrm{Cl}_{2}$, rt, $18 \mathrm{~h}, 78 \%$ over 2 steps. (iii) $\mathrm{Ph}_{3} \mathrm{COH}, \mathrm{CF}_{3} \mathrm{CO}_{2} \mathrm{H}, \mathrm{CH}_{2} \mathrm{Cl}_{2}$, rt, $4 \mathrm{~h}$. (iv) Boc-Leu-OH, HOBt $\cdot \mathrm{H}_{2} \mathrm{O}, \mathrm{EDCl} \cdot \mathrm{HCl}, \mathrm{CH}_{2} \mathrm{Cl}_{2}$, rt, $16 \mathrm{~h}, 88 \%$ over 2 steps. (v) $\mathrm{Ph}_{3} \mathrm{COH}, \mathrm{CF}_{3} \mathrm{CO}_{2} \mathrm{H}$, $\mathrm{CH}_{2} \mathrm{Cl}_{2}$, rt, $2.5 \mathrm{~h}$. (vi) Boc-Thi-OH, $\mathrm{HOBt} \cdot \mathrm{H}_{2} \mathrm{O}, \mathrm{EDCl} \cdot \mathrm{HCl}, \mathrm{CH}_{2} \mathrm{Cl}_{2}$, rt, $16 \mathrm{~h}, 87 \%$ over 2 steps. (vii) TIPSH, $\mathrm{CF}_{3} \mathrm{CO}_{2} \mathrm{H}, \mathrm{CH}_{2} \mathrm{Cl}_{2}$, rt, 30 min. (viii) $\mathrm{Ph}_{3} \mathrm{COH}, \mathrm{CF}_{3} \mathrm{CO}_{2} \mathrm{H}, \mathrm{CH}_{2} \mathrm{Cl}_{2}$, rt, 1.0 h. (ix) Boc-Cys(Trt)-OH, $\mathrm{HOBt} \cdot \mathrm{H}_{2} \mathrm{O}, \mathrm{EDCl} \cdot \mathrm{HCl}, \mathrm{CH}_{2} \mathrm{Cl}_{2}$, rt, $16 \mathrm{~h}, 76 \%$ over 2 steps. (x) TIPSH, $\mathrm{CF}_{3} \mathrm{CO}_{2} \mathrm{H}, \mathrm{CH}_{2} \mathrm{Cl}_{2}$, rt, 30 min. 
S13

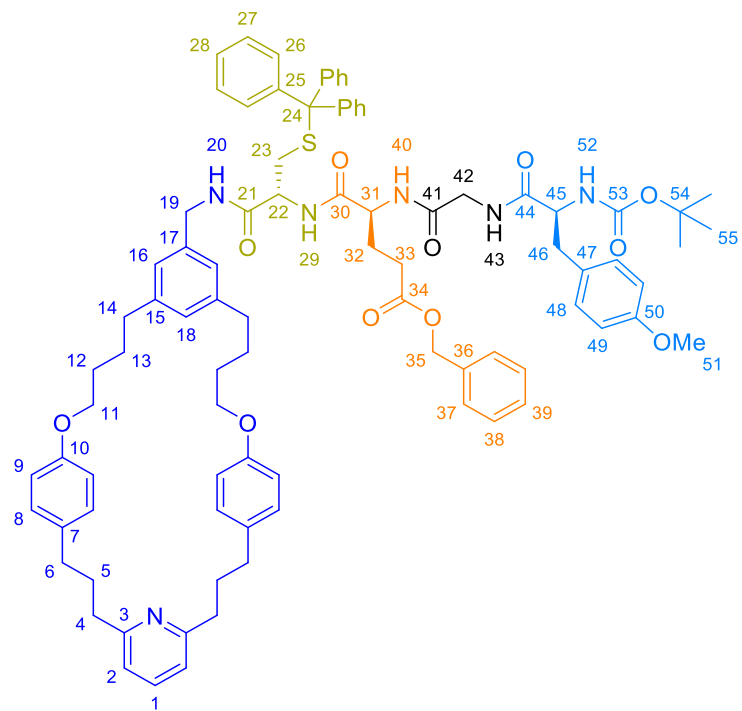

Macrocycle S8 (250 mg, $0.19 \mathrm{mmol}, 1.0$ equiv.) and triphenylmethanol ( $25 \mathrm{mg}, 0.10 \mathrm{mmol}, 0.5$ equiv.) were dissolved in a mixture of $\mathrm{CH}_{2} \mathrm{Cl}_{2}: \mathrm{CF}_{3} \mathrm{CO}_{2} \mathrm{H}(9: 1,4 \mathrm{~mL})$. The solution was stirred at room temperature for $4 \mathrm{~h}$ before toluene $(8 \mathrm{~mL})$ was added and the mixture was concentrated to dryness under reduced pressure to give a foam solid. The crude salt was dissolved in $\mathrm{CH}_{2} \mathrm{Cl}_{2}$ and washed with saturated aqueous $\mathrm{NaHCO}_{3}$ and then with brine. The collected organic phase was dried over $\mathrm{MgSO}_{4}$, filtered, and the solvent was reduced under reduced pressure to afford the crude as an off-white foam that was taken to the next reaction without further purification.

Boc-Tyr(Me)-OH (63 mg, $0.21 \mathrm{mmol}, 1.1$ equiv.), $\mathrm{EDCl} \cdot \mathrm{HCl}(37 \mathrm{mg}, 0.19 \mathrm{mmol}, 1.0$ equiv.) and $\mathrm{HOBt} \cdot \mathrm{H}_{2} \mathrm{O}$ (30 mg, $0.19 \mathrm{mmol}, 1.0$ equiv.) were added to the crude residue $(263 \mathrm{mg}$, assume $0.19 \mathrm{mmol}, 1.0$ equiv.) and the mixture was dissolved in $\mathrm{CH}_{2} \mathrm{Cl}_{2}(4 \mathrm{~mL})$ and stirred at room temperature for $18 \mathrm{~h}$. The crude was purified by flash column chromatography $\left(\mathrm{SiO}_{2}\right.$, $\mathrm{CH}_{2} \mathrm{Cl}_{2} / \mathrm{MeOH} 100: 1$ to $20: 1$ ) to yield $\mathbf{S} 13$ as a colorless solid (221 $\mathrm{mg}, 0.15 \mathrm{mmol}, 78 \%$ ).

${ }^{1} \mathrm{H}$ NMR $\left(600 \mathrm{MHz}, \mathrm{DMSO}-d_{6}\right) \delta 8.42\left(\mathrm{t}, J=6.0 \mathrm{~Hz}, 1 \mathrm{H}, \mathrm{H}_{20}\right), 8.22\left(\mathrm{~d}, J=8.0 \mathrm{~Hz}, 1 \mathrm{H}, \mathrm{H}_{29}\right)$, $8.18\left(\mathrm{t}, J=5.7 \mathrm{~Hz}, 1 \mathrm{H}, \mathrm{H}_{43}\right), 8.03\left(\mathrm{~d}, J=7.8 \mathrm{~Hz}, 1 \mathrm{H}, \mathrm{H}_{40}\right), 7.58\left(\mathrm{t}, J=7.6 \mathrm{~Hz}, 1 \mathrm{H}, \mathrm{H}_{1}\right), 7.34-$ $7.25\left(\mathrm{~m}, 17 \mathrm{H}, \mathrm{H}_{26}+\mathrm{H}_{27}+\mathrm{H}_{37}+\mathrm{H}_{38}+\mathrm{H}_{39}\right), 7.23-7.20\left(\mathrm{~m}, 3 \mathrm{H}, \mathrm{H}_{28}\right), 7.14(\mathrm{~d}, J=8.3 \mathrm{~Hz}, 2 \mathrm{H}$, $\left.\mathrm{H}_{48}\right), 7.06\left(\mathrm{~d}, J=7.6 \mathrm{~Hz}, 2 \mathrm{H}, \mathrm{H}_{2}\right), 7.02\left(\mathrm{~d}, J=8.6 \mathrm{~Hz}, 4 \mathrm{H}, \mathrm{H}_{8}\right), 6.91-6.90\left(\mathrm{~m}, 2 \mathrm{H}, \mathrm{H}_{52}+\mathrm{H}_{18}\right)$, $6.84\left(\mathrm{~d}, J=1.5 \mathrm{~Hz}, 2 \mathrm{H}, \mathrm{H}_{18}\right), 6.80\left(\mathrm{~d}, J=8.5 \mathrm{~Hz}, 2 \mathrm{H}, \mathrm{H}_{49}\right), 6.72\left(\mathrm{~d}, J=8.5 \mathrm{~Hz}, 4 \mathrm{H}, \mathrm{H}_{9}\right), 5.05$ (s, $\left.2 \mathrm{H}, \mathrm{H}_{35}\right), 4.38-4.29\left(\mathrm{~m}, 3 \mathrm{H}, \mathrm{H}_{19}, \mathrm{H}_{22}+\mathrm{H}_{31}\right), 4.13-4.07\left(\mathrm{~m}, 2 \mathrm{H}, \mathrm{H}_{19}, \mathrm{H}_{45}\right), 3.84(\mathrm{t}, J=5.9$ $\left.\mathrm{Hz}, 4 \mathrm{H}, \mathrm{H}_{11}\right), 3.79-3.71\left(\mathrm{~m}, 2 \mathrm{H}, \mathrm{H}_{42}\right), 3.69\left(\mathrm{~s}, 3 \mathrm{H}, \mathrm{H}_{51}\right), 2.91\left(\mathrm{dd}, J=14.0,4.2 \mathrm{~Hz}, 1 \mathrm{H}, \mathrm{H}_{46}\right)$, $2.69\left(\mathrm{t}, J=7.3 \mathrm{~Hz}, 4 \mathrm{H}, \mathrm{H}_{4}\right), 2.67-2.60\left(\mathrm{~m}, 1 \mathrm{H}, \mathrm{H}_{46^{\prime}}\right), 2.49-2.45\left(\mathrm{~m}, 8 \mathrm{H}, \mathrm{H}_{6}+\mathrm{H}_{14}\right), 2.44-$ $2.34\left(\mathrm{~m}, 4 \mathrm{H}, \mathrm{H}_{23}+\mathrm{H}_{33}\right), 1.98-1.89\left(\mathrm{~m}, 5 \mathrm{H}, \mathrm{H}_{5}+\mathrm{H}_{32}\right), 1.83-1.75\left(\mathrm{~m}, 1 \mathrm{H}, \mathrm{H}_{32}\right), 1.66-1.55$ $\left(\mathrm{m}, 8 \mathrm{H}, \mathrm{H}_{12}+\mathrm{H}_{13}\right), 1.27$ (s, 9H, $\left.\mathrm{H}_{55}\right) ;{ }^{13} \mathrm{C}$ NMR $\left(151 \mathrm{MHz}\right.$, DMSO- $\left.d_{6}\right) \delta 172.19,172.17,170.7$, $169.4,168.9,160.5,157.7,156.8,155.3,144.2$, 141.8, 138.8, 136.7, 136.1, 133.9, 130.2, $130.1,129.14,129.05,128.4,128.1,128.0,127.9,126.8,126.5,124.6,120.2,114.1,113.4$, 
78.3, 67.0, 65.9, 65.5, 56.0, 54.9, 51.9, 51.7, 42.12, 42.06, 40.0, 36.6, 34.4, 33.7, 31.4, 30.0, 28.1, 27.9, 27.8, 27.6; HRMS (ESI') Calculated for $\mathrm{C}_{89} \mathrm{H}_{100} \mathrm{~N}_{6} \mathrm{O}_{11} \mathrm{SCl}[\mathrm{M}+\mathrm{CI}]^{-} 1495.6833$, found 1495.6847.

S14

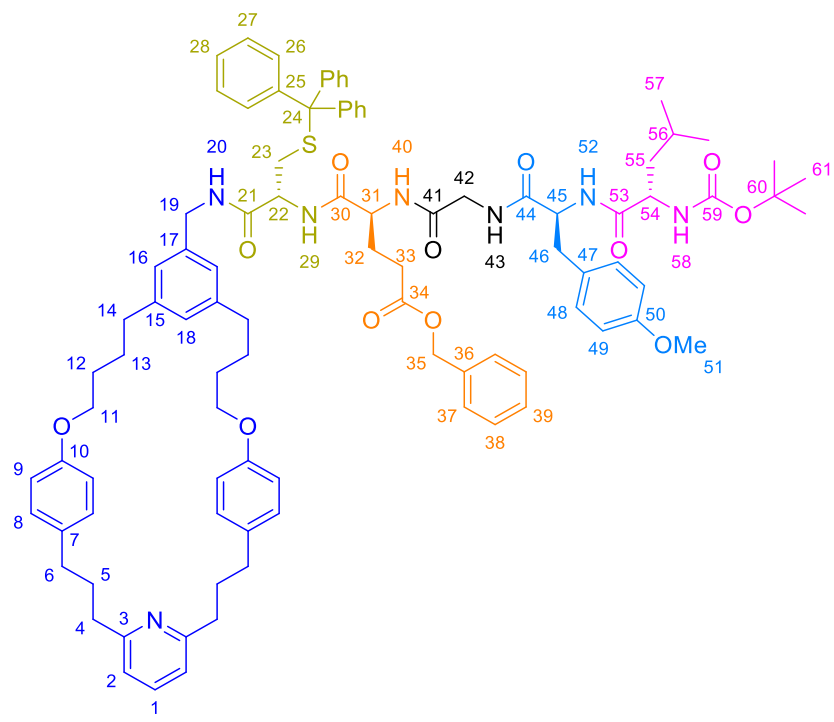

Macrocycle S13 (213 mg, $0.14 \mathrm{mmol}, 1.0$ equiv.) and triphenylmethanol (19 mg, $0.07 \mathrm{mmol}$, 0.5 equiv.) were dissolved in a mixture of $\mathrm{CH}_{2} \mathrm{Cl}_{2}: \mathrm{CF}_{3} \mathrm{CO}_{2} \mathrm{H}(9: 1,3 \mathrm{~mL})$. The solution was stirred at room temperature for $4 \mathrm{~h}$ before toluene $(6 \mathrm{~mL})$ was added and the mixture was concentrated under reduced pressure to dryness to give a foamy solid. The crude salt was dissolved in $\mathrm{CH}_{2} \mathrm{Cl}_{2}$ and washed with saturated aqueous $\mathrm{NaHCO}_{3}$ and then with brine. The collected organic phase was dried over $\mathrm{MgSO}_{4}$, filtered, and the solvent was removed under reduced pressure to afford the crude an off-white solid that was taken to the next reaction without further purification.

Boc-Leu-OH (63 mg, $0.16 \mathrm{mmol}, 1.1$ equiv.), $\mathrm{EDCl} \cdot \mathrm{HCl}(28 \mathrm{mg}, 0.14 \mathrm{mmol}, 1.0$ equiv.) and HOBt $\cdot \mathrm{H}_{2} \mathrm{O}$ (22 mg, $0.14 \mathrm{mmol}, 1.0$ equiv.) were added to the crude residue (226 $\mathrm{mg}$, assume $0.14 \mathrm{mmol}, 1.0$ equiv.) and the mixture was dissolved in $\mathrm{CH}_{2} \mathrm{Cl}_{2}(7 \mathrm{~mL})$ and stirred at room temperature for $16 \mathrm{~h}$. The reaction was diluted with $\mathrm{CH}_{2} \mathrm{Cl}_{2}$, washed with saturated aqueous $\mathrm{NaHCO}_{3}$ and then with brine. The collected organic phase was dried over $\mathrm{MgSO}_{4}$, filtered, and the solvent was removed under reduced pressure to afford a crude that was purified by flash column chromatography $\left(\mathrm{SiO}_{2}, \mathrm{CH}_{2} \mathrm{Cl}_{2} / \mathrm{MeOH} 100: 1\right.$ to $\left.50: 1\right)$ to yield $\mathbf{S 1 4}$ as an off-white solid (203 mg, $0.13 \mathrm{mmol}, 88 \%$ ).

${ }^{1} \mathrm{H}$ NMR $\left(600 \mathrm{MHz}, \mathrm{DMSO}-d_{6}\right) \delta 8.43\left(\mathrm{t}, \mathrm{J}=6.0 \mathrm{~Hz}, 1 \mathrm{H}, \mathrm{H}_{20}\right), 8.28-8.22\left(\mathrm{~m}, 2 \mathrm{H}, \mathrm{H}_{29}\right.$ and $\left.\mathrm{H}_{43}\right)$, $8.03\left(\mathrm{~d}, J=7.9 \mathrm{~Hz}, 1 \mathrm{H}, \mathrm{H}_{40}\right), 7.75\left(\mathrm{~d}, J=8.1 \mathrm{~Hz}, 1 \mathrm{H}, \mathrm{H}_{52}\right), 7.58\left(\mathrm{t}, J=7.6 \mathrm{~Hz}, 1 \mathrm{H}, \mathrm{H}_{1}\right), 7.35-$ $7.26\left(\mathrm{~m}, 17 \mathrm{H}, \mathrm{H}_{26}+\mathrm{H}_{27}+\mathrm{H}_{37}+\mathrm{H}_{38}+\mathrm{H}_{39}\right), 7.25-7.19\left(\mathrm{~m}, 3 \mathrm{H}, \mathrm{H}_{28}\right), 7.11(\mathrm{~d}, J=8.3 \mathrm{~Hz}, 2 \mathrm{H}$, $\left.\mathrm{H}_{48}\right), 7.06\left(\mathrm{~d}, J=7.6 \mathrm{~Hz}, 2 \mathrm{H}, \mathrm{H}_{2}\right), 7.02\left(\mathrm{~d}, J=8.6 \mathrm{~Hz}, 4 \mathrm{H}, \mathrm{H}_{8}\right), 6.91\left(\mathrm{~s}, 1 \mathrm{H}, \mathrm{H}_{18}\right), 6.87(\mathrm{~d}, J=$ $\left.8.4 \mathrm{~Hz}, 1 \mathrm{H}, \mathrm{H}_{58}\right), 6.84\left(\mathrm{~d}, J=1.5 \mathrm{~Hz}, 2 \mathrm{H}, \mathrm{H}_{16}\right), 6.76\left(\mathrm{~d}, J=8.6 \mathrm{~Hz}, 2 \mathrm{H}, \mathrm{H}_{49}\right), 6.72(\mathrm{~d}, J=8.6$ 
$\left.\mathrm{Hz}, 4 \mathrm{H}, \mathrm{H}_{9}\right), 5.05\left(\mathrm{~s}, 2 \mathrm{H}, \mathrm{H}_{35}\right), 4.50-4.45\left(\mathrm{~m}, 1 \mathrm{H}, \mathrm{H}_{45}\right), 4.40-4.28\left(\mathrm{~m}, 3 \mathrm{H}, \mathrm{H}-19, \mathrm{H}_{22}+\mathrm{H}_{31}\right)$, $4.11\left(\mathrm{dd}, J=15.5,5.5 \mathrm{~Hz}, 1 \mathrm{H}, \mathrm{H}_{19}\right), 3.90-3.86\left(\mathrm{~m}, 1 \mathrm{H}, \mathrm{H}_{54}\right), 3.84\left(\mathrm{t}, J=5.9 \mathrm{~Hz}, 4 \mathrm{H}, \mathrm{H}_{11}\right), 3.79$ $-3.70\left(\mathrm{~m}, 2 \mathrm{H}, \mathrm{H}_{42}\right), 3.68\left(\mathrm{~s}, 3 \mathrm{H}, \mathrm{H}_{51}\right), 2.94\left(\mathrm{dd}, \mathrm{J}=13.9,4.5 \mathrm{~Hz}, 1 \mathrm{H}, \mathrm{H}_{46}\right), 2.75-2.71(\mathrm{~m}, 1 \mathrm{H}$, $\left.\mathrm{H}_{46^{\prime}}\right), 2.69\left(\mathrm{t}, J=7.4 \mathrm{~Hz}, 4 \mathrm{H}, \mathrm{H}_{4}\right), 2.49-2.45\left(\mathrm{~m}, 8 \mathrm{H}, \mathrm{H}_{6}+\mathrm{H}_{14}\right), 2.45-2.33\left(\mathrm{~m}, 4 \mathrm{H}, \mathrm{H}_{23}+\mathrm{H}_{33}\right)$, $1.96-1.88\left(\mathrm{~m}, 5 \mathrm{H}, \mathrm{H}_{5}+\mathrm{H}_{32}\right), 1.86-1.76\left(\mathrm{~m}, 1 \mathrm{H}, \mathrm{H}_{32}\right), 1.66-1.55\left(\mathrm{~m}, 8 \mathrm{H}, \mathrm{H}_{12}+\mathrm{H}_{13}\right), 1.38$ $-1.36\left(\mathrm{~m}, 1 \mathrm{H}, \mathrm{H}_{56}\right), 1.34\left(\mathrm{~s}, 9 \mathrm{H}, \mathrm{H}_{61}\right), 1.30-1.19\left(\mathrm{~m}, 2 \mathrm{H}, \mathrm{H}_{55}\right), 0.80\left(\mathrm{~d}, J=6.6 \mathrm{~Hz}, 3 \mathrm{H}, \mathrm{H}_{57}\right)$, $0.76\left(\mathrm{~d}, J=6.5 \mathrm{~Hz}, 3 \mathrm{H}, \mathrm{H}_{57}\right) ;{ }^{13} \mathrm{C}$ NMR $\left(151 \mathrm{MHz}\right.$, DMSO-d $\left.\mathrm{d}_{6}\right) \delta 172.23,172.18,171.4,170.6$, $169.4,168.7,160.5,157.7,156.8,155.2$, 144.2, 141.8, 138.8, 136.7, 136.1, 133.9, 130.3, 129.2, 129.1, 128.4, 128.1, 128.0, 127.9, 126.8, 126.5, 124.7, 120.2, 114.1, 113.3, 78.1, 67.0, $65.9,65.5,54.9,53.7,53.1,51.8,51.7,42.1,41.9,40.9,40.1,36.6,34.4,33.7,31.4,30.0$, 28.2, 27.9, 27.64, 27.57, 24.1, 22.8, 21.6; HRMS $\left(\mathrm{ESI}^{+}\right)$Calculated for $\mathrm{C}_{95} \mathrm{H}_{111} \mathrm{~N}_{7} \mathrm{O}_{12} \mathrm{SNa}$ $[\mathrm{M}+\mathrm{Na}]^{+} 1597.7982$, found 1597.7954 .

\section{S15}

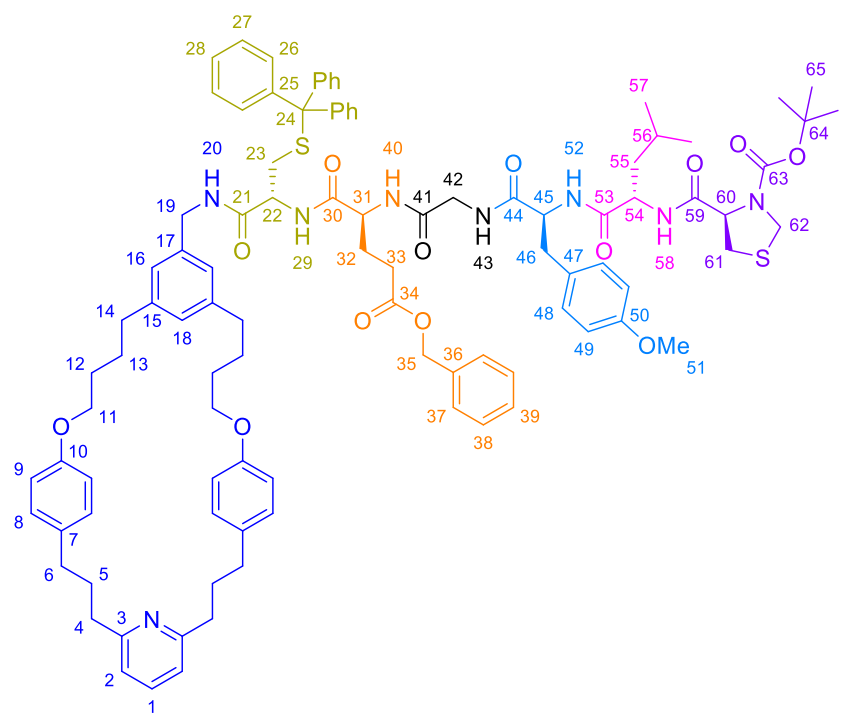

Macrocycle S14 (160 mg, $0.10 \mathrm{mmol}, 1.0$ equiv.) and triphenylmethanol (13 mg, $0.05 \mathrm{mmol}$, 0.5 equiv.) were dissolved in a mixture of $\mathrm{CH}_{2} \mathrm{Cl}_{2}: \mathrm{CF}_{3} \mathrm{CO}_{2} \mathrm{H}(9: 1,3 \mathrm{~mL})$. The solution was stirred at room temperature for $2.5 \mathrm{~h}$ before toluene $(7 \mathrm{~mL})$ was added and the mixture was concentrated to dryness under reduced pressure to give a foamy solid. The crude salt was dissolved in $\mathrm{CH}_{2} \mathrm{Cl}_{2}$ and washed with saturated aqueous $\mathrm{NaHCO}_{3}$ and then with brine. The collected organic phase was dried over $\mathrm{MgSO}_{4}$, filtered, and the solvent was removed under reduced pressure to afford the crude an off-white solid that was taken to the next reaction without further purification.

Boc-Thi-OH (26 mg, $0.11 \mathrm{mmol}, 1.1$ equiv.), EDCl. $\mathrm{HCl}(20 \mathrm{mg}, 0.10 \mathrm{mmol}, 1.0$ equiv.) and $\mathrm{HOBt} \cdot \mathrm{H}_{2} \mathrm{O}$ (16 mg, $0.10 \mathrm{mmol}, 1.0$ equiv.) were added to the crude residue (173 $\mathrm{mg}$, assume $0.10 \mathrm{mmol}, 1.0$ equiv.) and the mixture was dissolved in $\mathrm{CH}_{2} \mathrm{Cl}_{2}(8 \mathrm{~mL})$ and stirred at room 
temperature for $16 \mathrm{~h}$. The reaction was diluted with $\mathrm{CH}_{2} \mathrm{Cl}_{2}$, washed with saturated aqueous $\mathrm{NaHCO}_{3}$ and then with brine. The collected organic phase was dried over $\mathrm{MgSO}_{4}$, filtered, and the solvent was removed under reduced pressure to afford a crude that was purified by flash column chromatography $\left(\mathrm{SiO}_{2}, \mathrm{CH}_{2} \mathrm{Cl}_{2} / \mathrm{MeOH} 100: 1\right.$ to $\left.50: 1\right)$ to yield $\mathbf{S 1 5}$ as an off-white solid (150 mg, $0.13 \mathrm{mmol}, 87 \%$ ).

${ }^{1} \mathrm{H}$ NMR $\left(600 \mathrm{MHz}, \mathrm{DMSO}-d_{6}\right) \delta 8.43\left(\mathrm{t}, J=6.1 \mathrm{~Hz}, 1 \mathrm{H}, \mathrm{H}_{20}\right), 8.25\left(\mathrm{~d}, J=7.9 \mathrm{~Hz}, 1 \mathrm{H}, \mathrm{H}_{29}\right)$, $8.21\left(\mathrm{~s}, 1 \mathrm{H}, \mathrm{H}_{43}\right), 8.03\left(\mathrm{~d}, J=7.8 \mathrm{~Hz}, 1 \mathrm{H}, \mathrm{H}_{40}\right), 7.98\left(\mathrm{~d}, J=8.2 \mathrm{~Hz}, 2 \mathrm{H}, \mathrm{H}_{52}+\mathrm{H}_{58}\right), 7.58(\mathrm{t}, J=$ $\left.7.6 \mathrm{~Hz}, 1 \mathrm{H}, \mathrm{H}_{1}\right), 7.35-7.25\left(\mathrm{~m}, 17 \mathrm{H}, \mathrm{H}_{26}+\mathrm{H}_{27}+\mathrm{H}_{37}+\mathrm{H}_{38}+\mathrm{H}_{39}\right), 7.23-7.20\left(\mathrm{~m}, 3 \mathrm{H}, \mathrm{H}_{28}\right)$, $7.11\left(\mathrm{~d}, J=8.4 \mathrm{~Hz}, 2 \mathrm{H}, \mathrm{H}_{48}\right), 7.06\left(\mathrm{~d}, J=7.6 \mathrm{~Hz}, 2 \mathrm{H}, \mathrm{H}_{2}\right), 7.02\left(\mathrm{~d}, J=8.5 \mathrm{~Hz}, 4 \mathrm{H}, \mathrm{H}_{8}\right), 6.91$ (s, $\left.1 \mathrm{H}, \mathrm{H}_{18}\right), 6.84\left(\mathrm{~s}, 2 \mathrm{H}, \mathrm{H}_{16}\right), 6.77\left(\mathrm{~d}, J=8.6 \mathrm{~Hz}, 2 \mathrm{H}, \mathrm{H}_{49}\right), 6.72\left(\mathrm{~d}, J=8.5 \mathrm{~Hz}, 4 \mathrm{H}, \mathrm{H}_{9}\right), 5.05$ (s, $\left.2 \mathrm{H}, \mathrm{H}_{35}\right), 4.56\left(\mathrm{~d}, J=9.0 \mathrm{~Hz}, 1 \mathrm{H}, \mathrm{H}_{62}\right), 4.51-4.42\left(\mathrm{~m}, 2 \mathrm{H}, \mathrm{H}_{60}+\mathrm{H}_{45}\right), 4.40-4.25\left(\mathrm{~m}, 5 \mathrm{H}, \mathrm{H}_{19}\right.$ $\left.+\mathrm{H}_{22}+\mathrm{H}_{31}+\mathrm{H}_{54}+\mathrm{H}_{62^{\prime}}\right), 4.10\left(\mathrm{dd}, J=15.5,5.5 \mathrm{~Hz}, 1 \mathrm{H}, \mathrm{H}_{19^{\prime}}\right), 3.84\left(\mathrm{t}, J=5.9 \mathrm{~Hz}, 4 \mathrm{H}, \mathrm{H}_{11}\right), 3.79$ $-3.70\left(\mathrm{~m}, 2 \mathrm{H}, \mathrm{H}_{42}\right), 3.68\left(\mathrm{~s}, 3 \mathrm{H}, \mathrm{H}_{51}\right), 3.35-3.32\left(\mathrm{~m}, 1 \mathrm{H}, \mathrm{H}_{61}\right), 2.95(\mathrm{dd}, J=14.1,4.6 \mathrm{~Hz}, 1 \mathrm{H}$, $\left.\mathrm{H}_{46}\right), 2.92-2.85\left(\mathrm{~m}, 1 \mathrm{H}, \mathrm{H}_{61^{\prime}}\right), 2.74\left(\mathrm{dd}, J=14.0,9.2 \mathrm{~Hz}, 1 \mathrm{H}, \mathrm{H}_{46^{\prime}}\right), 2.69\left(\mathrm{t}, J=7.3 \mathrm{~Hz}, 4 \mathrm{H}, \mathrm{H}_{4}\right)$, $2.49-2.45\left(\mathrm{~m}, 8 \mathrm{H}, \mathrm{H}_{6}+\mathrm{H}_{14}\right), 2.45-2.33\left(\mathrm{~m}, 4 \mathrm{H}, \mathrm{H}_{23}+\mathrm{H}_{33}\right), 1.97-1.88\left(\mathrm{~m}, 5 \mathrm{H}, \mathrm{H}_{5}+\mathrm{H}_{32}\right)$, $1.84-1.76\left(\mathrm{~m}, 1 \mathrm{H}, \mathrm{H}_{32}\right), 1.67-1.51\left(\mathrm{~m}, 9 \mathrm{H}, \mathrm{H}_{12}+\mathrm{H}_{13}+\mathrm{H}_{56}\right), 1.42-1.27\left(\mathrm{~m}, 11 \mathrm{H}, \mathrm{H}_{55}+\mathrm{H}_{65}\right)$, $0.85-0.75\left(\mathrm{~m}, 6 \mathrm{H}, \mathrm{H}_{57}\right) ;{ }^{13} \mathrm{C}$ NMR $\left(151 \mathrm{MHz}\right.$, DMSO- $\left.d_{6}\right) \delta 172.2,171.7,171.3,170.6,169.9$, $169.4,168.7,160.5,157.8,156.8,152.7,144.2,141.8,138.8,136.7,136.1,133.9,130.2$, $129.5,129.2,129.1,128.4,128.1,128.0,127.9,126.8,126.6,124.7,120.2,114.1,113.4,79.8$, $67.0,65.9,65.5,61.4,54.9,54.0,51.8,51.7,51.1,49.5,42.1,42.0,41.0,40.1,36.6,35.1$, $34.4,33.7,31.4,27.9,27.8,27.65,27.57,24.0,23.1,21.6$; HRMS $\left(\mathrm{ESI}^{+}\right)$Calculated for $\mathrm{C}_{99} \mathrm{H}_{117} \mathrm{~N}_{8} \mathrm{O}_{13} \mathrm{~S}_{2}[\mathrm{M}+\mathrm{H}]^{+}$1689.8176, found 1689.8186 . 


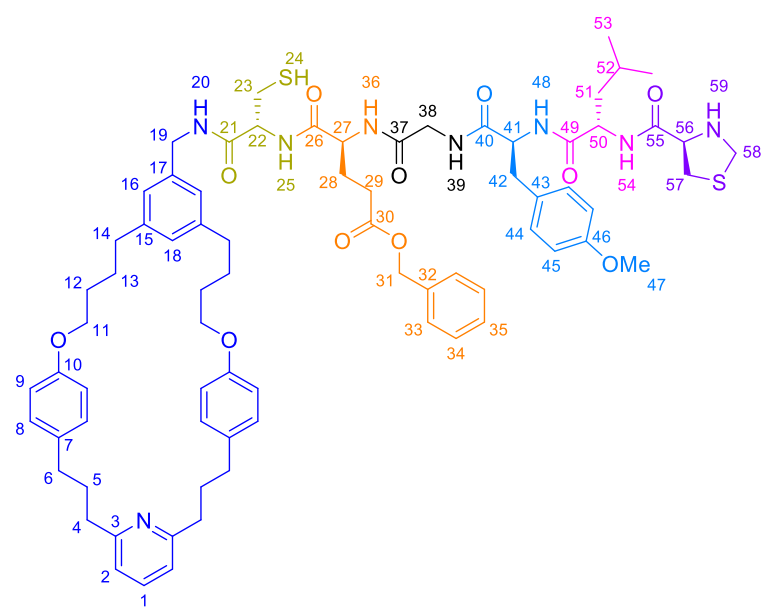

Compound $\mathbf{S} 15$ (10 mg, $5.9 \mu \mathrm{mol}$, 1 eq.) was dissolved in anhydrous $\mathrm{CH}_{2} \mathrm{Cl}_{2}(0.8 \mathrm{~mL})$, $\mathrm{CF}_{3} \mathrm{CO}_{2} \mathrm{H}(0.2 \mathrm{~mL})$ was added followed immediately by TIPSH $(5.9 \mu \mathrm{L})$ after which the yellow solution became colourless in ca. $5 \mathrm{~min}$. The resulting solution was stirred for $30 \mathrm{~min}$ at room temperature. Toluene $(2.0 \mathrm{~mL})$ was added and the solution concentrated to dryness under reduced pressure. The resulting solid was further azeotroped with toluene $(2 \times 2.0 \mathrm{~mL})$ under reduced pressure. The resulting solid was triturated with $\mathrm{Et}_{2} \mathrm{O}$ :pentane $(1: 2,3 \times 2.0 \mathrm{~mL})$. The remaining solid was dried thoroughly under reduced pressure to give the crude deprotected product 7 in quantitative yield.

${ }^{1} \mathrm{H}$ NMR $\left(600 \mathrm{MHz}, \mathrm{DMSO}-d_{6}\right) \delta 8.47\left(\mathrm{t}, J=6.0 \mathrm{~Hz}, 1 \mathrm{H}, \mathrm{H}_{20}\right), 8.25\left(\mathrm{t}, J=5.6 \mathrm{~Hz}, 1 \mathrm{H}, \mathrm{H}_{39}\right), 8.20$ (d, $\left.J=8.0 \mathrm{~Hz}, 1 \mathrm{H}, \mathrm{H}_{25}\right), 8.12\left(\mathrm{~d}, J=8.0 \mathrm{~Hz}, 1 \mathrm{H}, \mathrm{H}_{48}\right), 8.07\left(\mathrm{~d}, J=7.7 \mathrm{~Hz}, 1 \mathrm{H}, \mathrm{H}_{36}\right), 7.37-7.26$ $\left(\mathrm{m}, 5 \mathrm{H}, \mathrm{H}_{33}+\mathrm{H}_{34}+\mathrm{H}_{35}\right), 7.15-7.10\left(\mathrm{~m}, 4 \mathrm{H}, \mathrm{H}_{2}+\mathrm{H}_{44}\right), 7.02\left(\mathrm{~d}, \mathrm{~J}=8.3 \mathrm{~Hz}, 4 \mathrm{H}, \mathrm{H}_{8}\right), 6.90(\mathrm{~s}$, $\left.3 \mathrm{H}, \mathrm{H}_{16}+\mathrm{H}_{18}\right), 6.78\left(\mathrm{~d}, J=8.5 \mathrm{~Hz}, 2 \mathrm{H}, \mathrm{H}_{45}\right), 6.74\left(\mathrm{~d}, J=8.3 \mathrm{~Hz}, 4 \mathrm{H}, \mathrm{H}_{9}\right), 5.07\left(\mathrm{~s}, 2 \mathrm{H}, \mathrm{H}_{31}\right)$, $4.50-4.44\left(\mathrm{td}, J=8.8,4.6 \mathrm{~Hz}, 1 \mathrm{H}, \mathrm{H}_{41}\right), 4.42-4.36\left(\mathrm{~m}, 2 \mathrm{H}, \mathrm{H}_{22}+\mathrm{H}_{27}\right), 4.35-4.16(\mathrm{~m}, 5 \mathrm{H}$, $\left.\mathrm{H}_{19}+\mathrm{H}_{50}+\mathrm{H}_{58}+\mathrm{H}_{19}\right), 3.87\left(\mathrm{t}, J=5.6 \mathrm{~Hz}, 4 \mathrm{H}, \mathrm{H}_{11}\right), 3.80-3.71\left(\mathrm{~m}, 2 \mathrm{H}, \mathrm{H}_{38}\right), 3.69\left(\mathrm{~s}, 3 \mathrm{H}, \mathrm{H}_{47}\right)$, $2.96\left(\mathrm{dd}, J=14.0,4.5 \mathrm{~Hz}, 1 \mathrm{H}, \mathrm{H}_{42}\right), 2.92\left(\mathrm{dd}, J=11.0,6.7 \mathrm{~Hz}, 1 \mathrm{H}, \mathrm{H}_{57}\right) 2.85-2.67(\mathrm{~m}, 6 \mathrm{H}$, $\left.\mathrm{H}_{23}+\mathrm{H}_{4}+\mathrm{H}_{42^{\prime}}\right), 2.49-2.45\left(\mathrm{~m}, 8 \mathrm{H}, \mathrm{H}_{6}+\mathrm{H}_{14}\right), 2.55-2.36\left(\mathrm{~m}, 4 \mathrm{H}, \mathrm{H}_{23^{\prime}}+\mathrm{H}_{29}+\mathrm{H}_{57^{\prime}}\right), 2.31(\mathrm{t}, J$ $\left.=8.4,1 \mathrm{H}, \mathrm{H}_{24}\right), 2.05-1.97\left(\mathrm{~m}, 1 \mathrm{H}, \mathrm{H}_{28}\right), 1.93\left(\mathrm{~m}, 4 \mathrm{H}, \mathrm{H}_{5}\right), 1.88-1.80\left(\mathrm{~m}, 1 \mathrm{H}, \mathrm{H}_{28}\right), 1.72-$ $1.59\left(\mathrm{~m}, 8 \mathrm{H}, \mathrm{H}_{12}+\mathrm{H}_{13}\right), 1.54-1.45\left(\mathrm{~m}, 1 \mathrm{H}, \mathrm{H}_{52}\right), 1.42-1.34\left(\mathrm{~m}, 2 \mathrm{H}, \mathrm{H}_{51}\right), 0.88-0.80(\mathrm{~m}, 6 \mathrm{H}$, $\left.\mathrm{H}_{53}\right) . \mathrm{H}_{1}, \mathrm{H}_{54}, \mathrm{H}_{56}$ and $\mathrm{H}_{59}$ can't be found due to the broadness and overlap of signals. $2 \mathrm{D}$ NMR techniques (COSY) were used to aid in the assignment of this compound.

LRMS $\left(\mathrm{ESI}^{+}\right)$Calculated for $\mathrm{C}_{75} \mathrm{H}_{95} \mathrm{~N}_{8} \mathrm{O}_{11} \mathrm{~S}_{2}[\mathrm{M}+\mathrm{H}]^{+} 1347.66$, found 1347.67 . 
S16

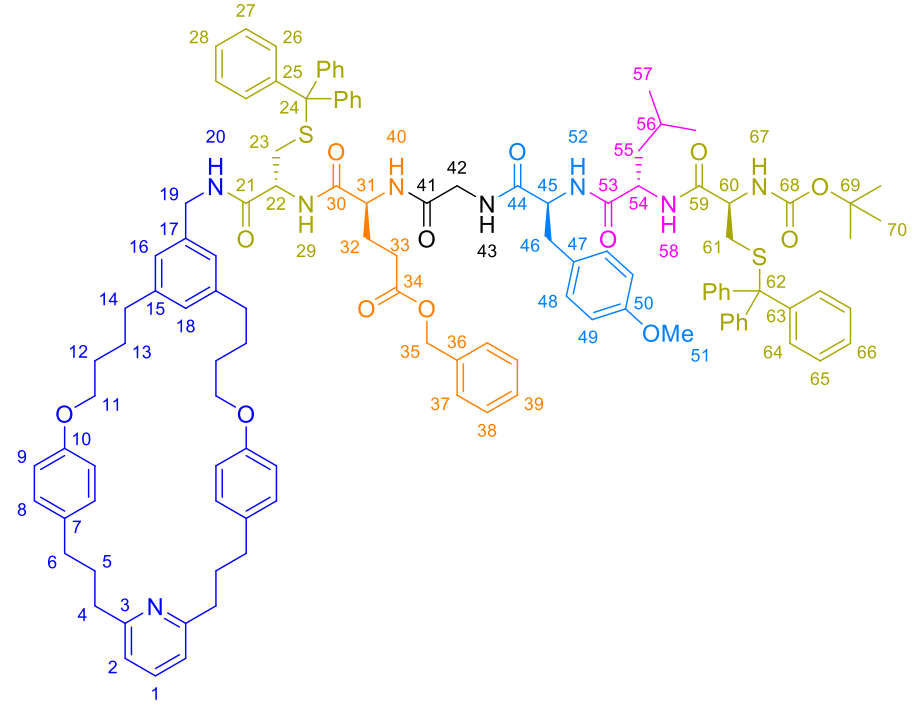

Macrocycle S14 (29 mg, $18.4 \mu \mathrm{mol}, 1.0$ equiv.) and triphenylmethanol (2.4 mg, $9.2 \mu \mathrm{mol}, 0.5$ equiv.) were dissolved in a mixture of $\mathrm{CH}_{2} \mathrm{Cl}_{2}: \mathrm{CF}_{3} \mathrm{CO}_{2} \mathrm{H}(4: 1,2.5 \mathrm{~mL})$. The solution was stirred at room temperature for $1 \mathrm{~h}$ before toluene $(5 \mathrm{~mL})$ was added and the mixture was concentrated to dryness under reduced pressure to give a foamy solid. The crude salt was dissolved in $\mathrm{CH}_{2} \mathrm{Cl}_{2}$ and washed with saturated aqueous $\mathrm{NaHCO}_{3}$ and then with brine. The collected organic phase was dried over $\mathrm{MgSO}_{4}$, filtered, and the solvent was removed under reduced pressure to afford the crude an off-white solid that was taken to the next reaction without further purification.

Boc-Cys(Trt)-OH (9 mg, $20.3 \mu \mathrm{mol}, 1.1$ equiv.), EDCl. $\mathrm{HCl}(3.5 \mathrm{mg}, 18.3 \mu \mathrm{mol}, 1.0$ equiv.) and $\mathrm{HOBt} \cdot \mathrm{H}_{2} \mathrm{O}$ (2.8 mg, $18.3 \mu \mathrm{mol}, 1.0$ equiv.) were added to the crude residue ( $30 \mathrm{mg}$, assume $18.3 \mu \mathrm{mol}, 1.0$ equiv. $)$ and the mixture was dissolved in $\mathrm{CH}_{2} \mathrm{Cl}_{2}(3 \mathrm{~mL})$ and stirred at room temperature for $16 \mathrm{~h}$. The solvent was removed under reduced pressure and the crude was purified by preparative scale TLC $\left(\mathrm{SiO}_{2}, 2000 \mu \mathrm{m}\right.$ Merck plates, loading the crude in $\mathrm{CHCl}_{3}$ and eluting twice with $\mathrm{CH}_{2} \mathrm{Cl}_{2} / \mathrm{MeOH} 95: 5$ ) to yield $\mathbf{S 1 6}$ as an off-white solid (27 mg, $14.1 \mu \mathrm{mol}$, $76 \%)$.

${ }^{1} \mathrm{H}$ NMR $\left(600 \mathrm{MHz}, \mathrm{DMSO}-d_{6}\right) \delta 8.43\left(\mathrm{t}, J=6.0 \mathrm{~Hz}, 1 \mathrm{H}, \mathrm{H}_{20}\right), 8.25\left(\mathrm{~d}, J=7.9 \mathrm{~Hz}, 1 \mathrm{H}, \mathrm{H}_{29}\right)$, $8.21-8.16\left(\mathrm{~m}, 1 \mathrm{H}, \mathrm{H}_{43}\right), 8.02\left(\mathrm{~d}, J=7.8 \mathrm{~Hz}, 1 \mathrm{H}, \mathrm{H}_{40}\right), 7.96\left(\mathrm{~d}, J=7.9 \mathrm{~Hz}, 1 \mathrm{H}, \mathrm{H}_{52}\right), 7.58(\mathrm{t}, J$ $\left.=7.6 \mathrm{~Hz}, 1 \mathrm{H}, \mathrm{H}_{1}\right), 7.50$ (d, J = 8.4 Hz, $\left.1 \mathrm{H}, \mathrm{H}_{58}\right), 7.35-7.25\left(\mathrm{~m}, 29 \mathrm{H}, \mathrm{H}_{26}, \mathrm{H}_{27}, \mathrm{H}_{64}, \mathrm{H}_{65}, \mathrm{H}_{37}\right.$, $\mathrm{H}_{38}$ and $\left.\mathrm{H}_{39}\right), 7.23-7.19\left(\mathrm{~m}, 6 \mathrm{H}, \mathrm{H}_{28}+\mathrm{H}_{66}\right), 7.10\left(\mathrm{~d}, \mathrm{~J}=8.6 \mathrm{~Hz}, 1 \mathrm{H}, \mathrm{H}_{67}\right), 7.08-7.04(\mathrm{~m}, 4 \mathrm{H}$, $\left.\mathrm{H}_{2}+\mathrm{H}_{48}\right), 7.02\left(\mathrm{~d}, J=8.2 \mathrm{~Hz}, 4 \mathrm{H}, \mathrm{H}_{8}\right), 6.91\left(\mathrm{~s}, 1 \mathrm{H}, \mathrm{H}_{18}\right), 6.84\left(\mathrm{~s}, 2 \mathrm{H}, \mathrm{H}_{16}\right), 6.74(\mathrm{~d}, J=8.5 \mathrm{~Hz}$, $\left.2 \mathrm{H}, \mathrm{H}_{49}\right), 6.71\left(\mathrm{~d}, J=8.5 \mathrm{~Hz}, 4 \mathrm{H}, \mathrm{H}_{9}\right), 5.05\left(\mathrm{~s}, 2 \mathrm{H}, \mathrm{H}_{35}\right), 4.46-4.40\left(\mathrm{~m}, 1 \mathrm{H}, \mathrm{H}_{45}\right), 4.40-4.22$ (m, $\left.4 \mathrm{H}, \mathrm{H}_{19}, \mathrm{H}_{22}, \mathrm{H}_{31}, \mathrm{H}_{54}\right), 4.10\left(\mathrm{dd}, J=15.6,5.4 \mathrm{~Hz}, 1 \mathrm{H}, \mathrm{H}_{19}\right), 3.88-3.81\left(\mathrm{~m}, 5 \mathrm{H}, \mathrm{H}_{11}+\mathrm{H}_{60}\right)$, $3.79-3.70\left(m, 1 \mathrm{H}, \mathrm{H}_{42}\right), 3.69-3.62\left(\mathrm{~m}, 4 \mathrm{H}, \mathrm{H}_{42}+\mathrm{H}_{51}\right), 2.92-2.87\left(\mathrm{~m}, 1 \mathrm{H}, \mathrm{H}_{46}\right), 2.73-2.65$ $\left(\mathrm{m}, 5 \mathrm{H}, \mathrm{H}_{46^{\prime}}+\mathrm{H}_{4}\right), 2.48-2.44\left(\mathrm{~m}, 8 \mathrm{H}, \mathrm{H}_{6}+\mathrm{H}_{14}\right), 2.43-2.33\left(\mathrm{~m}, 5 \mathrm{H}, \mathrm{H}_{23}+\mathrm{H}_{33}+\mathrm{H}_{61}\right), 2.32-$ 
$2.25\left(\mathrm{~m}, 1 \mathrm{H}, \mathrm{H}_{61}\right), 1.97-1.87\left(\mathrm{~m}, 5 \mathrm{H}, \mathrm{H}_{5}+\mathrm{H}_{32}\right), 1.84-1.75\left(\mathrm{~m}, 1 \mathrm{H}, \mathrm{H}_{32^{\prime}}\right), 1.66-1.55(\mathrm{~m}, 8 \mathrm{H}$, $\mathrm{H}_{12}$ and $\left.\mathrm{H}_{13}\right), 1.49-1.40\left(\mathrm{~m}, 1 \mathrm{H}, \mathrm{H}_{56}\right), 1.36\left(\mathrm{~s}, 9 \mathrm{H}, \mathrm{H}_{70}\right), 1.33-1.22\left(\mathrm{~m}, 2 \mathrm{H}, \mathrm{H}_{55}\right), 0.75(\mathrm{~d}, \mathrm{~J}=$ $\left.6.6 \mathrm{~Hz}, 3 \mathrm{H}, \mathrm{H}_{57}\right), 0.71\left(\mathrm{~d}, J=6.5 \mathrm{~Hz}, 3 \mathrm{H}, \mathrm{H}_{57}\right) ;{ }^{13} \mathrm{C} \mathrm{NMR}\left(151 \mathrm{MHz}, \mathrm{CDCl}_{3}\right) \delta 173.20,173.01$, $172.50,171.28,170.27,169.87,161.54,158.58,157.35,156.24,144.84,144.12,142.40$, $138.80,136.63,136.03,134.52,129.79,129.54,129.38,128.64,128.42,128.21,128.06$, 127.36, 127.02, 126.77, 125.46, 120.26, 114.48, 114.10, 81.39, 67.85, 67.74, 67.11, 66.45, $55.75,55.37,54.23,54.01,53.58,53.19,44.06,43.39,39.87,38.08,35.18,34.81,33.54$, $32.59,32.07,31.00,29.85,28.69,28.27,28.01,26.21,24.87,22.93,21.75,14.28$; HRMS $\left(E S I^{+}\right)$Calculated for $\mathrm{C}_{117} \mathrm{H}_{131} \mathrm{O}_{13} \mathrm{~N}_{8} \mathrm{~S}_{2}[\mathrm{M}+\mathrm{H}]^{+}$1919.9272, found 1919.9275 .

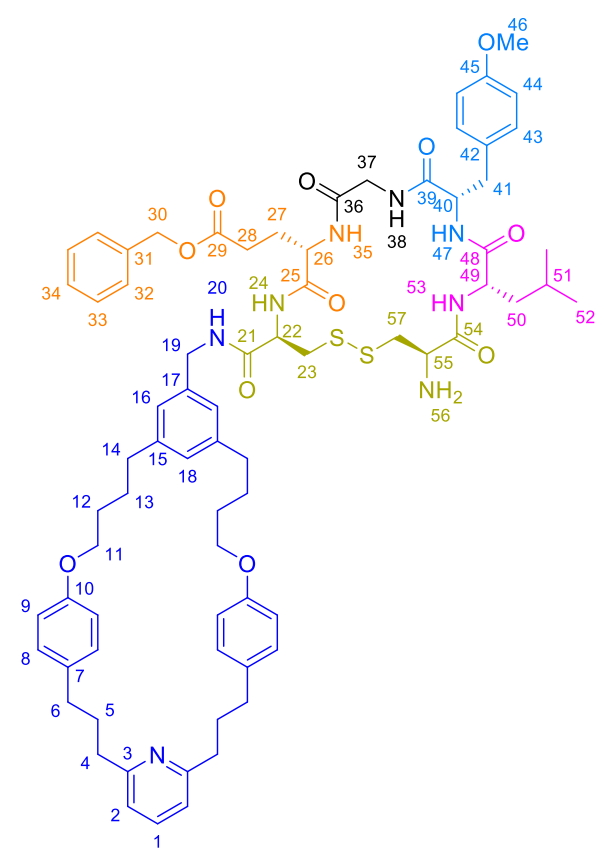

Macrocycle $\mathbf{S} 16$ (22 mg, $11.5 \mu \mathrm{mol}, 1.0$ equiv.) was dissolved in a mixture of $\mathrm{CH}_{2} \mathrm{Cl}_{2}: \mathrm{CF}_{3} \mathrm{CO}_{2} \mathrm{H}$ $(4: 1,0.8 \mathrm{~mL})$ and then TIPSH (3 drops) was added. The reaction was stirred at $\mathrm{rt}$ for $30 \mathrm{~min}$. Toluene $(0.5 \mathrm{~mL})$ was added and the mixture was concentrated to dryness under reduced pressure. The crude was treated with a mixture of $n$-hexane: $\mathrm{Et}_{2} \mathrm{O}(2: 1)$ and filtered. The crude salt was dissolved in $\mathrm{CH}_{2} \mathrm{Cl}_{2}$ and washed with saturated aqueous $\mathrm{NaHCO}_{3}$ and then with brine. The collected organic phase was dried over $\mathrm{MgSO}_{4}$, filtered, and the solvent was removed under reduced pressure to afford $13 \mathrm{mg}$ of crude 9 as an off-white solid that was carried through without further purification (85\%, containing less than $10 \%$ impurity of leftover $\mathrm{Ph}_{3} \mathrm{CH}$ ). The deprotected product 9 slowly formed the corresponding disulfide $\mathbf{S 1 7}$ and was characterized as such.

${ }^{1} \mathrm{H}$ NMR $\left(600 \mathrm{MHz}, \mathrm{DMSO}-\mathrm{d}_{6}\right) \delta 8.55\left(\mathrm{~d}, J=6.5 \mathrm{~Hz}, 1 \mathrm{H}, \mathrm{H}_{53}\right), 8.52-8.40\left(\mathrm{~m}, 2 \mathrm{H}, \mathrm{H}_{20}+\mathrm{H}_{47}\right)$, $8.27\left(\mathrm{dd}, J=13.7,7.0 \mathrm{~Hz}, 2 \mathrm{H}, \mathrm{H}_{24}+\mathrm{H}_{38}\right), 7.82\left(\mathrm{~d}, J=8.2 \mathrm{~Hz}, 1 \mathrm{H}, \mathrm{H}_{35}\right), 7.58(\mathrm{t}, J=7.6 \mathrm{~Hz}, 1 \mathrm{H}$, 
$\left.\mathrm{H}_{1}\right), 7.36-7.24\left(\mathrm{~m}, 6 \mathrm{H}, \mathrm{H}_{32}+\mathrm{H}_{33}+\mathrm{H}_{34}\right), 7.21\left(\mathrm{t}, J=7.4 \mathrm{~Hz}, P h_{3} \mathrm{CH}\right), 7.11(\mathrm{~d}, J=7.7 \mathrm{~Hz}$, $P h_{3} \mathrm{CH}$ ), $7.08-7.04\left(\mathrm{~m}, 4 \mathrm{H}, \mathrm{H}_{43}+\mathrm{H}_{2}\right), 7.02\left(\mathrm{~d}, \mathrm{~J}=8.3 \mathrm{~Hz}, 4 \mathrm{H}, \mathrm{H}_{8}\right), 6.93\left(\mathrm{~s}, 1 \mathrm{H}, \mathrm{H}_{18}\right), 6.88$ (s, $\left.2 \mathrm{H}, \mathrm{H}_{16}\right), 6.81\left(\mathrm{~d}, J=8.2 \mathrm{~Hz}, 2 \mathrm{H}, \mathrm{H}_{44}\right), 6.72\left(\mathrm{~d}, J=8.3 \mathrm{~Hz}, 4 \mathrm{H}, \mathrm{H}_{9}\right), 5.62\left(\mathrm{~s}, \mathrm{Ph}_{3} \mathrm{CH}\right), 5.05(\mathrm{~s}$, $\left.2 \mathrm{H}, \mathrm{H}_{30}\right), 4.57\left(\mathrm{tt}, J=13.3,6.3 \mathrm{~Hz}, 1 \mathrm{H}, \mathrm{H}_{22}\right), 4.49-4.33\left(\mathrm{~m}, 2 \mathrm{H}, \mathrm{H}_{26}+\mathrm{H}_{55}\right), 4.30-4.18(\mathrm{~d}, J=$ $\left.6.0 \mathrm{~Hz}, 2 \mathrm{H}, \mathrm{H}_{19}\right), 4.14-4.06\left(\mathrm{~m}, 2 \mathrm{H}, \mathrm{H}_{40}+\mathrm{H}_{49}\right), 3.92-3.80\left(\mathrm{~m}, 6 \mathrm{H}, \mathrm{H}_{11}+\mathrm{H}_{37}+\mathrm{H}_{57}\right), 3.70(\mathrm{~s}$, $\left.4 \mathrm{H}, \mathrm{H}_{46}+\mathrm{H}_{57^{\prime}}\right), 3.52-3.44\left(\mathrm{~m}, 1 \mathrm{H}, \mathrm{H}_{37}\right), 3.16\left(\mathrm{dd}, J=13.8,4.1 \mathrm{~Hz}, 1 \mathrm{H}, \mathrm{H}_{23}\right), 3.11(\mathrm{dd}, J=$ 13.8, $\left.5.1 \mathrm{~Hz}, 1 \mathrm{H}, \mathrm{H}_{41}\right), 3.01\left(\mathrm{dd}, J=13.7,9.3 \mathrm{~Hz}, 1 \mathrm{H}, \mathrm{H}_{23}\right), 2.92(\mathrm{dd}, J=14.1,9.9 \mathrm{~Hz}, 1 \mathrm{H}$, $\mathrm{H}_{41^{\prime}}$ ), $2.69\left(\mathrm{t}, J=7.4 \mathrm{~Hz}, 4 \mathrm{H}, \mathrm{H}_{4}\right), 2.57\left(\mathrm{t}, J=7.1 \mathrm{~Hz}, 4 \mathrm{H}, \mathrm{H}_{14} / \mathrm{H}_{6}\right), 2.47(\mathrm{t}, J=8.1 \mathrm{~Hz}, 4 \mathrm{H}$, $\left.\mathrm{H}_{14} / \mathrm{H}_{6}\right), 2.43-2.36\left(\mathrm{~m}, 1 \mathrm{H}, \mathrm{H}_{28}\right), 2.36-2.26\left(\mathrm{~m}, 1 \mathrm{H}, \mathrm{H}_{28}\right.$ ), 2.06 (tq, $J=18.4,7.6,5.5 \mathrm{~Hz}, 1 \mathrm{H}$, $\left.\mathrm{H}_{27}\right), 2.01-1.94\left(\mathrm{~m}, 1 \mathrm{H}, \mathrm{H}_{27^{\prime}}\right), 1.92\left(\mathrm{t}, \mathrm{J}=7.7 \mathrm{~Hz}, 4 \mathrm{H}, \mathrm{H}_{5}\right), 1.72-1.57\left(\mathrm{~m}, 8 \mathrm{H}, \mathrm{H}_{12}+\mathrm{H}_{13}\right), 1.43$ $\left(\mathrm{p}, J=6.7 \mathrm{~Hz}, 1 \mathrm{H}, \mathrm{H}_{51}\right), 1.40-1.24\left(\mathrm{~m}, 2 \mathrm{H}, \mathrm{H}_{50}\right), 0.80\left(\mathrm{~d}, J=6.6 \mathrm{~Hz}, 3 \mathrm{H}, \mathrm{H}_{52}\right), 0.77(\mathrm{~d}, J=6.4$ $\left.\mathrm{Hz}, 3 \mathrm{H}, \mathrm{H}_{52^{2}}\right) ;{ }^{13} \mathrm{C}$ NMR $(151 \mathrm{MHz}$, DMSO-d $) \delta 172.53,172.43,172.26,171.09,170.98$, $169.45,169.31,160.56,157.83,156.76,143.80,141.87,138.88,136.67,136.16,133.94$, $130.10,129.90,129.17,129.06,128.38,127.92,127.85,127.78,126.68,126.28,124.67$, $120.18,114.09,113.53,67.02,65.41,55.76,55.46,54.99,52.23,52.13,51.55,42.85,42.08$, $40.58,36.56,34.39,33.70,31.40,29.86,27.90,27.59,26.19,23.86,22.68,12.08$; HRMS $\left(E S I^{+}\right)$Calculated for $\mathrm{C}_{74} \mathrm{H}_{93} \mathrm{~N}_{8} \mathrm{O}_{11} \mathrm{~S}_{2}[\mathrm{M}+\mathrm{H}]^{+}$1333.6400, found 1333.6382 . 


\subsubsection{Synthesis of 8 and 10}
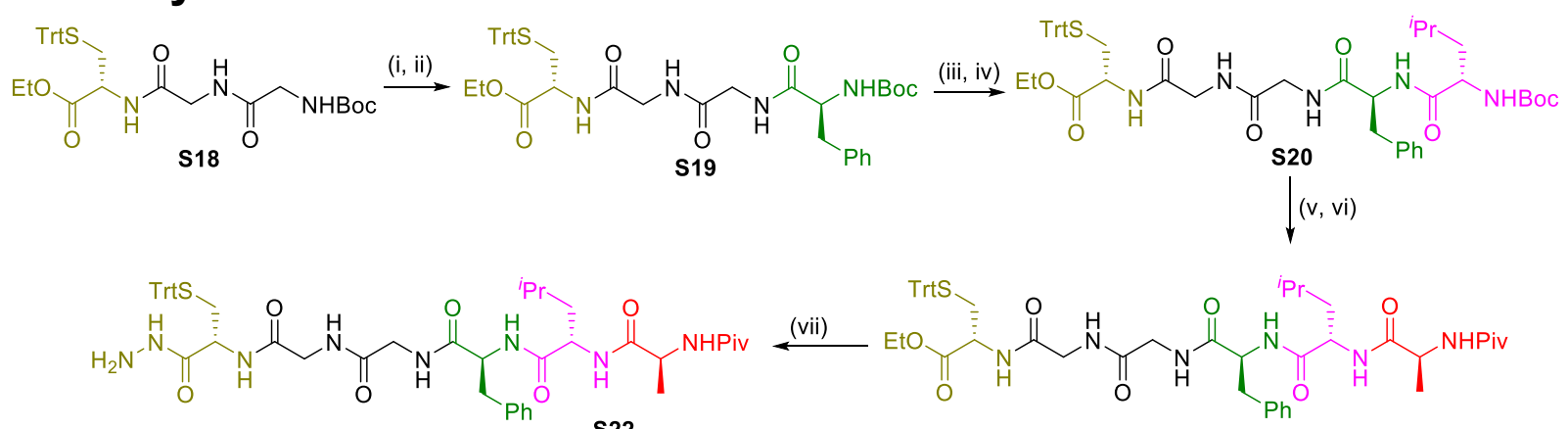

S22

S21

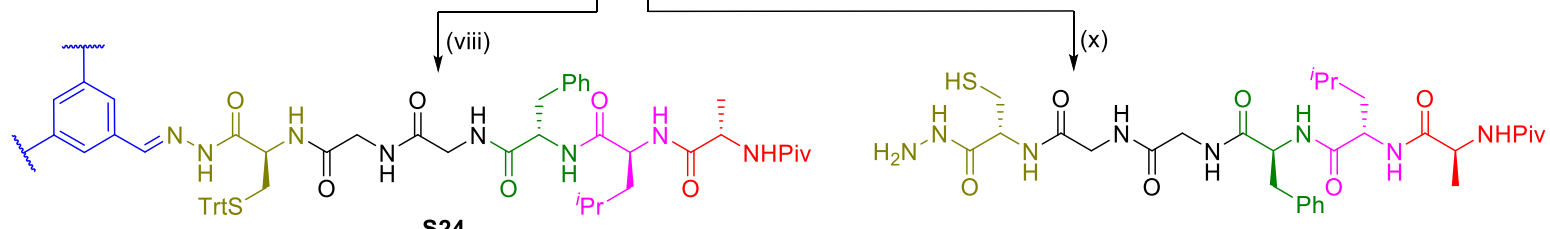

S24

(ix)
10

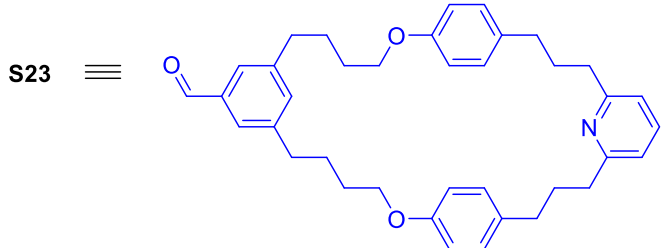

Scheme S12. Synthesis of standards 8 and 10. The hydrazide standard 10 was synthesised via deprotection from intermediate $\mathbf{S 2 2}$, and not from macrocycle $\mathbf{8}$, in order to allow for a clearer comparison in the ${ }^{1} \mathrm{H}$ NMR stackplot spectrum in Figure 2 (see main article). Reagents and conditions: (i) $\mathrm{Ph}_{3} \mathrm{COH}, \mathrm{CF}_{3} \mathrm{CO}_{2} \mathrm{H}, \mathrm{CH}_{2} \mathrm{Cl}_{2}$, rt, $2 \mathrm{~h}$; (ii) Boc-Phe-OH, $\mathrm{HOBt} \cdot \mathrm{H}_{2} \mathrm{O}, \mathrm{EDCl} \cdot \mathrm{HCl}, \mathrm{CH}_{2} \mathrm{Cl}_{2}, \mathrm{rt}, 16 \mathrm{~h}, 95 \%$ over 2 steps; (iii) $\mathrm{Ph}_{3} \mathrm{COH}, \mathrm{CF}_{3} \mathrm{CO}_{2} \mathrm{H}, \mathrm{CH}_{2} \mathrm{Cl}_{2}$, rt, 2 h; (iv) Boc-Leu-OH, HOBt $\cdot \mathrm{H}_{2} \mathrm{O}, \mathrm{EDCl} \cdot \mathrm{HCl}, \mathrm{CH}_{2} \mathrm{Cl}_{2}$, rt, 16 h, $85 \%$ over 2 steps; (v) $\mathrm{Ph}_{3} \mathrm{COH}, \mathrm{CF}_{3} \mathrm{CO}_{2} \mathrm{H}, \mathrm{CH}_{2} \mathrm{Cl}_{2}$, rt, 2 h; (vi) Piv-Ala-OH, $\mathrm{HOBt} \cdot \mathrm{H}_{2} \mathrm{O}, \mathrm{EDCl} \cdot \mathrm{HCl}$, $\mathrm{CH}_{2} \mathrm{Cl}_{2}$, rt, $16 \mathrm{~h}, 91 \%$ over 2 steps; (vii) $\mathrm{NH}_{2} \mathrm{NH}_{2}$, EtOH, rt, 16 h; (viii) Aldehyde macrocycle S23, aniline, $\mathrm{CH}_{2} \mathrm{Cl}_{2}, 40{ }^{\circ} \mathrm{C}$, 2 days, $82 \%$ over 2 steps. (ix) TIPSH, $\mathrm{CF}_{3} \mathrm{CO}_{2} \mathrm{H}, \mathrm{CH}_{2} \mathrm{Cl}_{2}$, rt, 15 min, quant. (x) TIPSH, $\mathrm{CF}_{3} \mathrm{CO}_{2} \mathrm{H}, \mathrm{CH}_{2} \mathrm{Cl}_{2}$, rt, 30 min, quant. 
S19

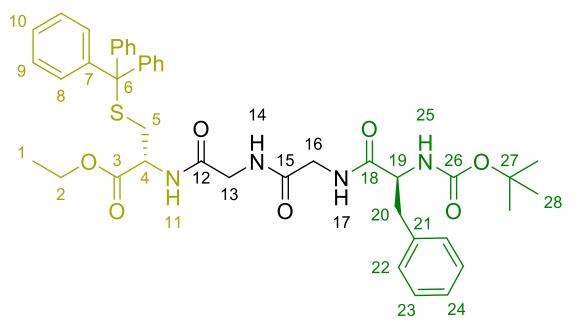

Prepared from S18 $(0.32 \mathrm{~g}, 0.53 \mathrm{mmol})$ using General Procedure $A$ and then General Procedure $B$. Purification by flash column chromatography $\left(\mathrm{SiO}, 5 \% \mathrm{MeOH} / \mathrm{CH}_{2} \mathrm{Cl}_{2}\right)$ yielded S19 as a colorless solid $(0.38 \mathrm{~g}, 0.51 \mathrm{mmol}, 95 \%$ over 2 steps).

${ }^{1} \mathrm{H}$ NMR $\left(600 \mathrm{MHz}, \mathrm{DMSO}-d_{6}\right) \delta 8.38\left(\mathrm{~d}, J=7.8 \mathrm{~Hz}, 1 \mathrm{H}, \mathrm{H}_{11}\right), 8.23\left(\mathrm{t}, J=5.7 \mathrm{~Hz}, 1 \mathrm{H}, \mathrm{H}_{17}\right)$, $8.01\left(\mathrm{t}, J=6.0 \mathrm{~Hz}, 1 \mathrm{H}, \mathrm{H}_{14}\right), 7.34\left(\mathrm{t}, J=7.7 \mathrm{~Hz}, 6 \mathrm{H}, \mathrm{H}_{9}\right), 7.29\left(\mathrm{~d}, J=7.5 \mathrm{~Hz}, 6 \mathrm{H}, \mathrm{H}_{8}\right), 7.28-$ $7.23\left(\mathrm{~m}, 7 \mathrm{H}, \mathrm{H}_{10}+\mathrm{H}_{22}+\mathrm{H}_{23}\right), 7.21-7.15\left(\mathrm{~m}, 1 \mathrm{H}, \mathrm{H}_{24}\right), 6.99\left(\mathrm{~d}, J=8.4 \mathrm{~Hz}, 1 \mathrm{H}, \mathrm{H}_{25}\right), 4.21-$ $4.15\left(\mathrm{~m}, 1 \mathrm{H}, \mathrm{H}_{19}\right), 4.09\left(\mathrm{td}, J=8.1,5.3 \mathrm{~Hz}, 1 \mathrm{H}, \mathrm{H}_{4}\right), 4.03-3.95\left(\mathrm{~m}, 2 \mathrm{H}, \mathrm{H}_{2}\right), 3.81-3.68(\mathrm{~m}$, $4 \mathrm{H}, \mathrm{H}_{13}+\mathrm{H}_{16}$ ), $3.00\left(\mathrm{dd}, J=13.9,4.3 \mathrm{~Hz}, 1 \mathrm{H}, \mathrm{H}_{20}\right.$ ), $2.72\left(\mathrm{dd}, J=13.8,10.4 \mathrm{~Hz}, 1 \mathrm{H}, \mathrm{H}_{20}\right), 2.55$ (dd, $\left.J=12.5,8.6 \mathrm{~Hz}, 1 \mathrm{H}, \mathrm{H}_{5}\right), 2.41-2.35\left(\mathrm{~m}, 1 \mathrm{H}, \mathrm{H}_{5}\right), 1.28\left(\mathrm{~s}, 9 \mathrm{H}, \mathrm{H}_{28}\right), 1.09(\mathrm{t}, J=7.1 \mathrm{~Hz}$, $\left.3 \mathrm{H}, \mathrm{H}_{1}\right) ;{ }^{13} \mathrm{C}$ NMR $\left(151 \mathrm{MHz}\right.$, DMSO- $\left.d_{6}\right) \delta 172.10,170.00,168.95,168.69,155.35,144.08$, 138.25, 129.22, 129.08, 128.15, 128.01, 126.90, 126.15, 78.10, 66.41, 60.83, 55.73, 51.58, 42.08, 41.38, 37.34, 32.82, 28.14, 13.95; HRMS (ESI ${ }^{+}$) Calculated for $\mathrm{C}_{42} \mathrm{H}_{48} \mathrm{~N}_{4} \mathrm{O}_{7} \mathrm{SNa}$ $[\mathrm{M}+\mathrm{Na}]^{+} 775.3136$, found 775.3139 .

\section{S20}

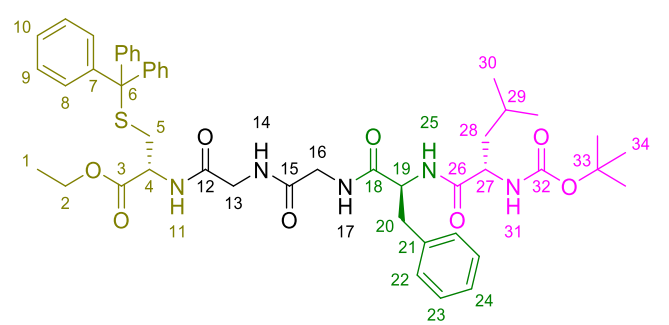

Prepared from S19 $(0.16 \mathrm{~g}, 0.217 \mathrm{mmol})$ using General Procedure $A$ and then General Procedure $B$. Purification by flash column chromatography $\left(\mathrm{SiO}, 5 \% \mathrm{MeOH} / \mathrm{CH}_{2} \mathrm{Cl}_{2}\right)$ yielded S20 as a colorless solid $(0.16 \mathrm{~g}, 0.185 \mathrm{mmol}, 85 \%$ over 2 steps).

${ }^{1} \mathrm{H}$ NMR $\left(600 \mathrm{MHz}\right.$, Acetonitrile- $\left.d_{3}\right) \delta 7.41-7.35\left(\mathrm{~m}, 7 \mathrm{H}, \mathrm{H}_{8}+\mathrm{H}_{17}\right), 7.31\left(\mathrm{t}, J=7.8 \mathrm{~Hz}, 6 \mathrm{H}, \mathrm{H}_{9}\right)$, $7.29-7.16\left(\mathrm{~m}, 9 \mathrm{H}, \mathrm{H}_{10}+\mathrm{H}_{22}+\mathrm{H}_{23}+\mathrm{H}_{24}\right), 7.11-7.04\left(\mathrm{~m}, 2 \mathrm{H}, \mathrm{H}_{11}+\mathrm{H}_{25}\right), 5.63(\mathrm{~d}, J=6.0 \mathrm{~Hz}$, $\left.1 \mathrm{H}, \mathrm{H}_{31}\right), 4.46\left(\mathrm{ddd}, J=9.3,7.1,5.3 \mathrm{~Hz}, 1 \mathrm{H}, \mathrm{H}_{19}\right), 4.09$ (q, $\left.J=7.8 \mathrm{~Hz}, 1 \mathrm{H}, \mathrm{H}_{4}\right), 4.06-3.99(\mathrm{~m}$, $\left.2 \mathrm{H}, \mathrm{H}_{2}\right), 3.87\left(\mathrm{td}, J=8.7,6.5,3.5 \mathrm{~Hz}, 1 \mathrm{H}, \mathrm{H}_{27}\right), 3.82-3.66\left(\mathrm{~m}, 4 \mathrm{H}, \mathrm{H}_{13}+\mathrm{H}_{16}\right), 3.19(\mathrm{dd}, J=$ 14.4, $\left.5.1 \mathrm{~Hz}, 1 \mathrm{H}, \mathrm{H}_{20}\right), 2.98$ (dd, $\left.J=14.1,9.2 \mathrm{~Hz}, 1 \mathrm{H}, \mathrm{H}_{20}\right), 2.68\left(\mathrm{dd}, J=12.8,8.5 \mathrm{~Hz}, 1 \mathrm{H}, \mathrm{H}_{5}\right.$ ), $2.49\left(\mathrm{dd}, J=12.8,4.9 \mathrm{~Hz}, 1 \mathrm{H}, \mathrm{H}_{5}\right), 1.54-1.44\left(\mathrm{~m}, 1 \mathrm{H}, \mathrm{H}_{29}\right), 1.36\left(\mathrm{~s}, 9 \mathrm{H}, \mathrm{H}_{34}\right), 1.35-1.28(\mathrm{~m}$, $\left.2 \mathrm{H}, \mathrm{H}_{28}\right), 1.13\left(\mathrm{t}, J=7.1 \mathrm{~Hz}, 3 \mathrm{H}, \mathrm{H}_{1}\right), 0.85\left(\mathrm{~d}, J=6.6 \mathrm{~Hz}, 3 \mathrm{H}, \mathrm{H}_{30}\right), 0.81(\mathrm{~d}, J=6.6 \mathrm{~Hz}, 3 \mathrm{H}$, $\left.\mathrm{H}_{30}\right) ;{ }^{13} \mathrm{C}$ NMR $\left(151 \mathrm{MHz}\right.$, Acetonitrile- $\left.d_{3}\right) \delta 174.74,173.27,171.10,170.19,169.94,157.41$, 
$145.47,138.44,130.37,130.20,129.39,129.06,127.88,127.63,80.46,67.70,62.21,55.83$, 54.83, 52.92, 44.14, 43.11, 41.16, 37.14, 34.02, 28.55, 25.33, 23.06, 21.94, 14.41; HRMS $\left(E S I^{+}\right)$Calculated for $\mathrm{C}_{48} \mathrm{H}_{59} \mathrm{~N}_{5} \mathrm{O}_{8} \mathrm{SNa}[\mathrm{M}+\mathrm{Na}]^{+} 888.3977$, found 888.3972.

S21

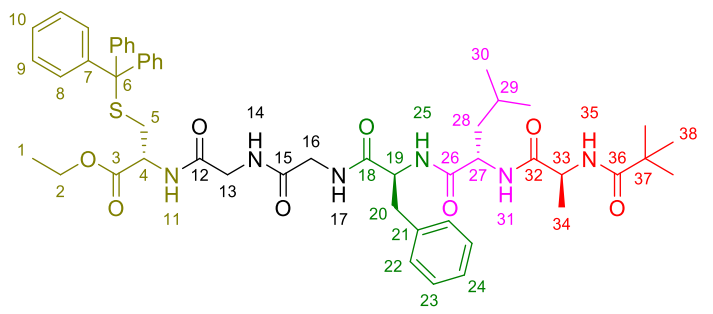

Prepared from S20 (0.22 g, $0.25 \mathrm{mmol})$ using General Procedure $A$ and then General Procedure $B$. Purification by flash column chromatography $\left(\mathrm{SiO}, 5 \% \mathrm{MeOH} / \mathrm{CH}_{2} \mathrm{Cl}_{2}\right)$ yielded S21 as a colorless solid $(0.21 \mathrm{~g}, 0.228 \mathrm{mmol}, 91 \%$ over 2 steps $)$.

${ }^{1} \mathrm{H}$ NMR $\left(600 \mathrm{MHz}, \mathrm{DMSO}-d_{6}\right) \delta 8.38\left(\mathrm{~d}, J=7.8 \mathrm{~Hz}, 1 \mathrm{H}, \mathrm{H}_{11}\right), 8.23\left(\mathrm{t}, J=5.7 \mathrm{~Hz}, 1 \mathrm{H}, \mathrm{H}_{17}\right)$, $7.96\left(\mathrm{t}, J=5.9 \mathrm{~Hz}, 1 \mathrm{H}, \mathrm{H}_{14}\right), 7.93\left(\mathrm{~d}, J=8.0 \mathrm{~Hz}, 1 \mathrm{H}, \mathrm{H}_{25}\right), 7.67\left(\mathrm{~d}, J=8.0 \mathrm{~Hz}, 1 \mathrm{H}, \mathrm{H}_{31}\right), 7.42$ (d, $\left.J=7.5 \mathrm{~Hz}, 1 \mathrm{H}, \mathrm{H}_{35}\right), 7.34\left(\mathrm{t}, J=7.6 \mathrm{~Hz}, 6 \mathrm{H}, \mathrm{H}_{9}\right), 7.29$ (d, J = 7.8 Hz, 6H, $\left.\mathrm{H}_{8}\right), 7.27-7.19$ $\left(\mathrm{m}, 7 \mathrm{H}, \mathrm{H}_{23}, \mathrm{H}_{22}+\mathrm{H}_{10}\right), 7.16\left(\mathrm{t}, J=7.0 \mathrm{~Hz}, 1 \mathrm{H}, \mathrm{H}_{24}\right), 4.52\left(\mathrm{td}, J=8.5,4.8 \mathrm{~Hz}, 1 \mathrm{H}, \mathrm{H}_{19}\right), 4.25$ (q, $\left.J=7.2 \mathrm{~Hz}, 1 \mathrm{H}, \mathrm{H}_{33}\right), 4.20$ (q, J = 7.9 Hz, 1H, $\mathrm{H}_{27}$ ), 4.10 (q, J = 7.8 Hz, 1H, $\mathrm{H}_{4}$ ), $4.03-3.95$ $\left(\mathrm{m}, 2 \mathrm{H}, \mathrm{H}_{2}\right), 3.78-3.69\left(\mathrm{~m}, 4 \mathrm{H}, \mathrm{H}_{13}+\mathrm{H}_{16}\right), 3.04\left(\mathrm{dd}, J=14.0,4.8 \mathrm{~Hz}, 1 \mathrm{H}, \mathrm{H}_{20}\right), 2.82(\mathrm{dd}, J=$ 14.0, 9.3 Hz, $\left.1 \mathrm{H}, \mathrm{H}_{20}\right), 2.55\left(\mathrm{dd}, J=12.5,8.4 \mathrm{~Hz}, 1 \mathrm{H}, \mathrm{H}_{5}\right), 2.42-2.35\left(\mathrm{~m}, 1 \mathrm{H}, \mathrm{H}_{5}\right), 1.48(\mathrm{dq}, J$ $\left.=13.3,6.8 \mathrm{~Hz}, 1 \mathrm{H}, \mathrm{H}_{29}\right), 1.34\left(\mathrm{t}, J=7.3 \mathrm{~Hz}, 2 \mathrm{H}, \mathrm{H}_{28}\right), 1.17\left(\mathrm{~d}, J=7.1 \mathrm{~Hz}, 3 \mathrm{H}, \mathrm{H}_{34}\right), 1.13-1.06$ $\left(\mathrm{m}, 12 \mathrm{H}, \mathrm{H}_{1}+\mathrm{H}_{38}\right), 0.82\left(\mathrm{~d}, J=6.6 \mathrm{~Hz}, 3 \mathrm{H}, \mathrm{H}_{30}\right), 0.79\left(\mathrm{~d}, J=6.5 \mathrm{~Hz}, 3 \mathrm{H}, \mathrm{H}_{30}\right) ;{ }^{13} \mathrm{C}$ NMR $(151$ $\left.\mathrm{MHz}, \mathrm{DMSO}-d_{6}\right) \delta 177.28,172.27,171.75,171.19,169.98,168.79,168.67,144.06,137.64$, $129.15,129.06,128.13,127.99,126.87,126.20$, 66.39, 60.81, 53.58, 51.57, 51.08, 48.17, $42.00,41.38,40.86,37.90,37.34,32.83,27.21,23.97,22.99,21.69,17.44,13.92$; HRMS $\left(\mathrm{ESI}^{+}\right.$) Calculated for $\mathrm{C}_{51} \mathrm{H}_{64} \mathrm{~N}_{6} \mathrm{O}_{8} \mathrm{SNa}[\mathrm{M}+\mathrm{Na}]^{+} 943.4399$, found 943.4430 . 
S22

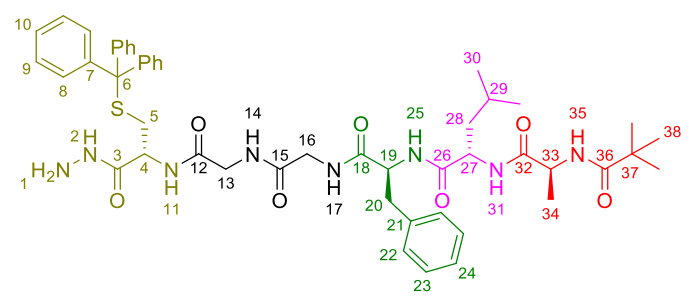

Hydrazine hydrate (64\% w/v in $\mathrm{H}_{2} \mathrm{O}, 29 \mu \mathrm{L}, 4.5$ equiv.) was added to a solution of $\mathbf{S 2 1}$ (115 $\mathrm{mg}, 0.126 \mathrm{mmol}, 1.0$ equiv. $)$ in ethanol $(2 \mathrm{~mL}, 50 \mathrm{mM})$ and stirred at room temperature for 16 h. The solvent was removed under reduced pressure and the solid azeotroped three times with toluene to obtain $\mathbf{S 2 2}$ as an orange solid that was used without further purification.

${ }^{1} \mathrm{H}$ NMR $\left(600 \mathrm{MHz}, \mathrm{DMSO}-d_{6}\right) \delta 9.16\left(\mathrm{~s}, 1 \mathrm{H}, \mathrm{H}_{2}\right), 8.25\left(\mathrm{t}, J=5.8 \mathrm{~Hz}, 1 \mathrm{H}, \mathrm{H}_{17}\right), 8.21$ (d, J=8.6 $\left.\mathrm{Hz}, \mathrm{H}_{11}\right), 7.98-7.92\left(\mathrm{~m}, 2 \mathrm{H}, \mathrm{H}_{14}+\mathrm{H}_{25}\right), 7.68\left(\mathrm{~d}, J=8.0 \mathrm{~Hz}, 1 \mathrm{H}, 1 \mathrm{H}, \mathrm{H}_{31}\right), 7.42(\mathrm{~d}, J=7.5 \mathrm{~Hz}$, $\left.\mathrm{H}_{35}\right), 7.37-7.30\left(\mathrm{~m}, 6 \mathrm{H}, \mathrm{H}_{9}\right), 7.29-7.25\left(\mathrm{~m}, 6 \mathrm{H}, \mathrm{H}_{8}\right), 7.25-7.22\left(\mathrm{~m}, 4 \mathrm{H}, \mathrm{H}_{22}+\mathrm{H}_{23}\right), 7.22-$ $7.19\left(\mathrm{~m}, 3 \mathrm{H}, \mathrm{H}_{10}\right), 7.19-7.13\left(\mathrm{~m}, 1 \mathrm{H}, \mathrm{H}_{24}\right), 4.52\left(\mathrm{td}, J=8.6,4.9 \mathrm{~Hz}, 1 \mathrm{H}, \mathrm{H}_{19}\right), 4.37(\mathrm{td}, J=8.4$, $\left.6.1 \mathrm{~Hz}, 1 \mathrm{H}, \mathrm{H}_{4}\right), 4.30-4.15\left(\mathrm{~m}, 4 \mathrm{H}, \mathrm{H}_{1}+\mathrm{H}_{27}+\mathrm{H}_{33}\right), 3.83-3.65\left(\mathrm{~m}, 4 \mathrm{H}, \mathrm{H}_{13}+\mathrm{H}_{16}\right), 3.04(\mathrm{dd}$, $\left.J=14.0,4.8 \mathrm{~Hz}, 1 \mathrm{H}, \mathrm{H}_{20}\right), 2.82\left(\mathrm{dd}, J=14.0,9.2 \mathrm{~Hz}, 1 \mathrm{H}, \mathrm{H}_{20^{\prime}}\right), 2.36(\mathrm{dd}, J=11.6,6.0 \mathrm{~Hz}, 1 \mathrm{H}$, $\left.\mathrm{H}_{5}\right), 2.34-2.28\left(\mathrm{~m}, 1 \mathrm{H}, \mathrm{H}_{5^{\prime}}\right), 1.49\left(\mathrm{dh}, J=13.5,6.7,6.3 \mathrm{~Hz}, 1 \mathrm{H}, \mathrm{H}_{29}\right), 1.34(\mathrm{t}, J=7.2 \mathrm{~Hz}, 2 \mathrm{H}$, $\left.\mathrm{H}_{28}\right), 1.17\left(\mathrm{~d}, J=7.1 \mathrm{~Hz}, 3 \mathrm{H}, \mathrm{H}_{34}\right), 1.08\left(\mathrm{~s}, 9 \mathrm{H}, \mathrm{H}_{38}\right), 0.83\left(\mathrm{~d}, J=6.6 \mathrm{~Hz}, 3 \mathrm{H}, \mathrm{H}_{30}\right), 0.79(\mathrm{~d}, J=$ $\left.6.4 \mathrm{~Hz}, 3 \mathrm{H}, \mathrm{H}_{30^{\circ}}\right) ;{ }^{13} \mathrm{C}$ NMR $\left(151 \mathrm{MHz}\right.$, DMSO- $\left.d_{6}\right) \delta 177.28,172.25,171.74,171.21,168.90$, $168.51,168.33,144.22,137.64,137.35,129.16,129.07,128.90,128.21,128.09,128.00$, $126.79,126.21,125.32,65.84,53.58,51.07,50.29,48.16,42.04,41.70,40.89,40.06,37.90$, 37.37, 34.03, 27.22, 23.97, 23.00, 21.69, 21.05, 17.45; HRMS (ESI ${ }^{+}$) Calculated for $\mathrm{C}_{49} \mathrm{H}_{62} \mathrm{~N}_{8} \mathrm{O}_{7} \mathrm{SNa}[\mathrm{M}+\mathrm{Na}]^{+}$929.4354, found 929.4371. 


\section{S24}

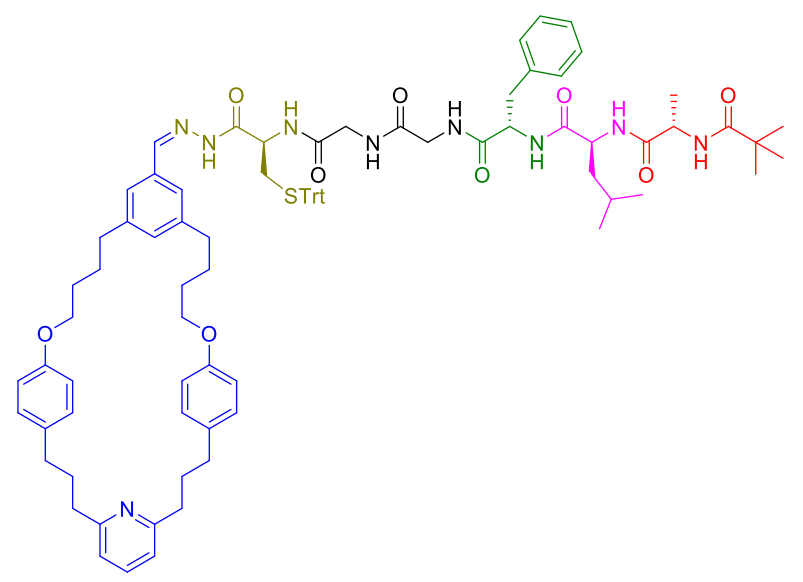

To crude hydrazide $\mathbf{S} 22$ (114mg, $0.126 \mathrm{mmol}, 1.0$ equiv.) in $\mathrm{CH}_{2} \mathrm{Cl}_{2}(5.0 \mathrm{~mL}$ ) was added aldehyde Macrocycle S23 (93 mg, $0.170 \mathrm{mmol}, 1.35$ equiv.) and aniline (5 drops). The resulting cloudy suspension was stirred for 2 days at $40^{\circ} \mathrm{C}$. The solvent was removed under reduced pressure and the crude residue purified by flash column chromatography $\left(\mathrm{SiO}_{2}, 5 \%\right.$ $\mathrm{MeOH} / \mathrm{CH}_{2} \mathrm{Cl}_{2}$ ) to give the title compound as a solid (150 mg, $0.103 \mathrm{mmol}, 82 \%$ ). Data consistent with that previously reported. ${ }^{2}$

8

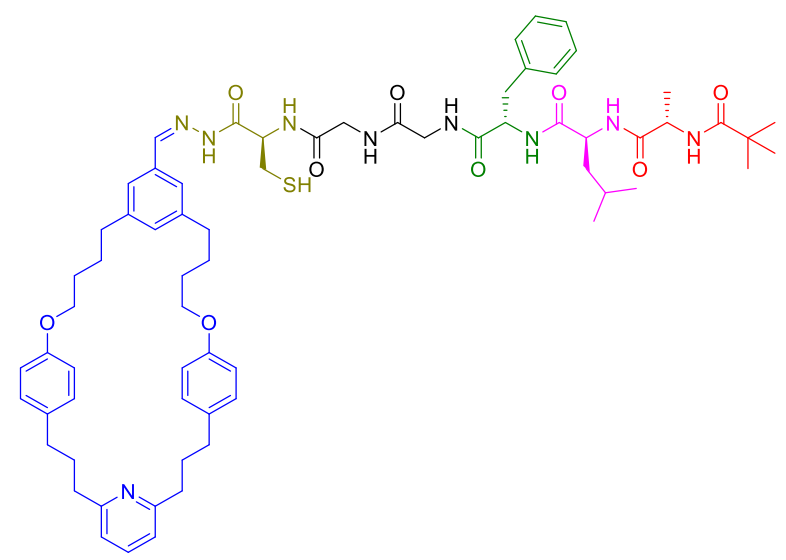

Compound $\mathbf{S 2 4}$ (20 mg, $13.4 \mu \mathrm{mol}, 1$ eq.) was dissolved in anhydrous $\mathrm{CH}_{2} \mathrm{Cl}_{2}(2.0 \mathrm{~mL})$, $\mathrm{CF}_{3} \mathrm{CO}_{2} \mathrm{H}(0.5 \mathrm{~mL})$ was added followed immediately by TIPSH (10 drops) after which the yellow solution became colourless in ca. $5 \mathrm{~min}$. The resulting solution was stirred for $15 \mathrm{~min}$ at room temperature. Toluene $(2.5 \mathrm{~mL})$ was added and the solution concentrated to dryness under reduced pressure. The resulting solid was further azeotroped with toluene $(2 \times 2.5 \mathrm{~mL})$ under reduced pressure. The resulting solid was triturated with $\mathrm{Et}_{2} \mathrm{O}: n$-hexane $(1: 1,2.5 \mathrm{~mL})$. The remaining solid was dried thoroughly under reduced pressure to give the crude deprotected product 8 in quantitative yield. Data consistent with that previously reported. ${ }^{2}$ 
10

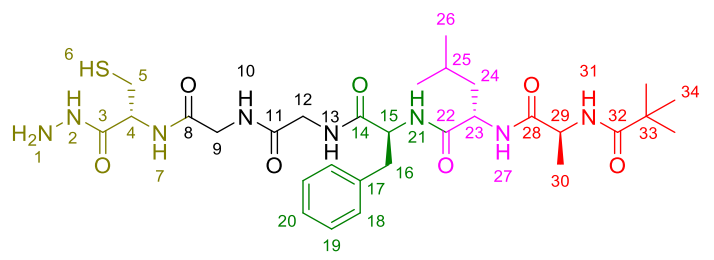

To crude hydrazide $\mathbf{S 2 2}$ (10 mg, $0.011 \mathrm{mmol}, 1.0$ equiv.) in $\mathrm{CH}_{2} \mathrm{Cl}_{2}(0.5 \mathrm{~mL})$ was added $\mathrm{CF}_{3} \mathrm{CO}_{2} \mathrm{H}(0.1 \mathrm{~mL})$ and TIPSH (10 drops). The reaction mixture was stirred $30 \mathrm{~min}$ at room temperature. The reaction mixture was diluted by $50 \%$ with toluene then the solvent was removed under reduced pressure. The crude residue was azeotroped twice with toluene to remove excess $\mathrm{CF}_{3} \mathrm{CO}_{2} \mathrm{H}$. Trituration with $\mathrm{Et}_{2} \mathrm{O}$ :n-hexane (1:1) yielded the product which was used without further purification.

${ }^{1} \mathrm{H}$ NMR $\left(600 \mathrm{MHz}, \mathrm{DMSO}-\mathrm{d}_{6}\right) \delta 10.05\left(\mathrm{~s}, 1 \mathrm{H}, \mathrm{H}_{2}\right), 8.28\left(\mathrm{t}, J=5.8 \mathrm{~Hz}, 1 \mathrm{H}, \mathrm{H}_{10}\right), 8.19(\mathrm{~d}, J=$ $\left.8.1 \mathrm{~Hz}, 1 \mathrm{H}, \mathrm{H}_{7}\right), 8.04\left(\mathrm{t}, J=5.8 \mathrm{~Hz}, 1 \mathrm{H}, \mathrm{H}_{13}\right), 7.96\left(\mathrm{~d}, J=8.0 \mathrm{~Hz}, 1 \mathrm{H}, \mathrm{H}_{21}\right), 7.69$ (d, J = 8.0 Hz, $\left.1 \mathrm{H}, \mathrm{H}_{27}\right), 7.44\left(\mathrm{~d}, J=7.5 \mathrm{~Hz}, 1 \mathrm{H}, \mathrm{H}_{31}\right), 7.27-7.15\left(\mathrm{~m}, 4 \mathrm{H}, \mathrm{H}_{18}+\mathrm{H}_{19}\right), 7.19-7.15\left(\mathrm{~m}, 1 \mathrm{H}, \mathrm{H}_{20}\right)$, $4.52\left(\mathrm{td}, J=8.5,4.7 \mathrm{~Hz}, 1 \mathrm{H}, \mathrm{H}_{15}\right), 4.39\left(\mathrm{td}, J=7.9,5.6 \mathrm{~Hz}, 1 \mathrm{H}, \mathrm{H}_{4}\right), 4.27-4.22\left(\mathrm{~m}, 1 \mathrm{H}, \mathrm{H}_{29}\right)$, $4.22-4.17\left(\mathrm{~m}, 1 \mathrm{H}, \mathrm{H}_{23}\right), 3.78\left(\mathrm{t}, J=6.0 \mathrm{~Hz}, 2 \mathrm{H}, \mathrm{H}_{9}\right), 3.73\left(\mathrm{t}, J=4.9 \mathrm{~Hz}, 2 \mathrm{H}, \mathrm{H}_{12}\right), 3.05(\mathrm{dd}, J$ $\left.=14.0,4.8 \mathrm{~Hz}, 1 \mathrm{H}, \mathrm{H}_{16}\right), 2.82\left(\mathrm{dd}, J=14.2,9.5 \mathrm{~Hz}, 1 \mathrm{H}, \mathrm{H}_{16}\right), 2.80-2.69\left(\mathrm{~m}, 2 \mathrm{H}, \mathrm{H}_{5}\right), 2.40(\mathrm{t}, J$ $\left.=8.5 \mathrm{~Hz}, 1 \mathrm{H}, \mathrm{H}_{6}\right), 1.48\left(\mathrm{dp}, J=13.4,6.8 \mathrm{~Hz}, 1 \mathrm{H}, \mathrm{H}_{25}\right), 1.34\left(\mathrm{t}, J=7.3 \mathrm{~Hz}, 2 \mathrm{H}, \mathrm{H}_{24}\right), 1.17(\mathrm{~d}, J$ $\left.=7.1 \mathrm{~Hz}, 3 \mathrm{H}, \mathrm{H}_{30}\right), 1.08\left(\mathrm{~s}, 9 \mathrm{H}, \mathrm{H}_{34}\right), 0.83\left(\mathrm{~d}, J=6.5 \mathrm{~Hz}, 3 \mathrm{H}, \mathrm{H}_{26}\right), 0.79(\mathrm{~d}, J=6.5 \mathrm{~Hz}, 3 \mathrm{H}$, $\mathrm{H}_{26}$ ). 2D NMR techniques (COSY, HSQC, HMBC) were used to aid in the assignment of this compound; ${ }^{13} \mathrm{C}$ NMR $\left(151 \mathrm{MHz}\right.$, DMSO- $\left.d_{6}\right) \delta 177.33,172.32,171.84,171.33,169.05,169.01$, $168.91,137.67,129.18,128.04,126.26,53.85,53.63,51.09,48.19,42.10,41.89$, 40.87, $37.93,37.33,27.23,25.88,23.98,23.03,21.71,17.44$; HRMS (ESI') Calculated for $\mathrm{C}_{30} \mathrm{H}_{47} \mathrm{O}_{7} \mathrm{~N}_{8} \mathrm{~S}: 663.3294[\mathrm{M}-\mathrm{H}]^{-}$, found 663.3299. 


\subsection{Ligation of operation products 7 and 8}<smiles>[3H][R17](=O)OC(=O)CC[C@H](NC(=O)CNC(=O)[C@H](Cc1ccc(OC)cc1)NC(=O)[C@H](CC(C)C)NC(=O)C1CSCN1)C(=O)N[C@@H](CS)C(=O)NCc1cc(C(C)C)cc(C(C)C)c1</smiles><smiles>CCCC(NC(=O)CNC(=O)CNC(=O)CNC(=O)CNC(=O)CNC(=O)CS)C(=O)N/N=C/c1cc(C(C)C)cc(C(C)C)c1</smiles><smiles>[CaH]</smiles><smiles>CCOC(=O)CCC(NC(=O)CNC(=O)C(Cc1ccc(OC)cc1)NC(=O)C(CC(C)C)NC(=O)C(N)CS)C(=O)N[C@@H](CS)C(=O)NCc1cc(C(C)C)cc(C(C)C)c1</smiles>

9

10

S25

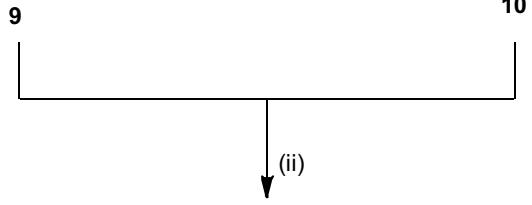<smiles>CCOC(=O)CCC(NC(=O)CNC(=O)C(Cc1ccc(OC)cc1)NC(=O)CC(CC(C)C)NC(=O)C(CS)NC(=O)C(CS)NC(=O)CNC(=O)CNC(=O)C(Cc1ccccc1)NC(=O)[C@H](CS)NC(=O)[C@H](CS)NC(=O)[C@H](C)NC(=O)NCc1cc(CC)cc(C(C)C)c1)C(C)C</smiles>

11

Scheme S13. Ligation of products of operation 7 and 8. Reagents and conditions: (i) $\mathrm{MeONH}_{2} \cdot \mathrm{HCl}$, $\mathrm{MeOH}, 50{ }^{\circ} \mathrm{C}, 3 \mathrm{~h}$, conversion assumed quant. (ii) $\mathrm{pH}=3, \mathrm{NaNO}_{2}, 6 \mathrm{M}$ guanidinium chloride, $0.2 \mathrm{M}$ $\mathrm{Na}_{2} \mathrm{HPO}_{4}, \mathrm{HFIP},-10{ }^{\circ} \mathrm{C}, 20 \mathrm{~min}$, then $\mathrm{pH}=7$, MPAA, 3h. Then TCEP.HCl, rt, $20 \mathrm{~min}, 25 \%$ over 2 steps. 
11

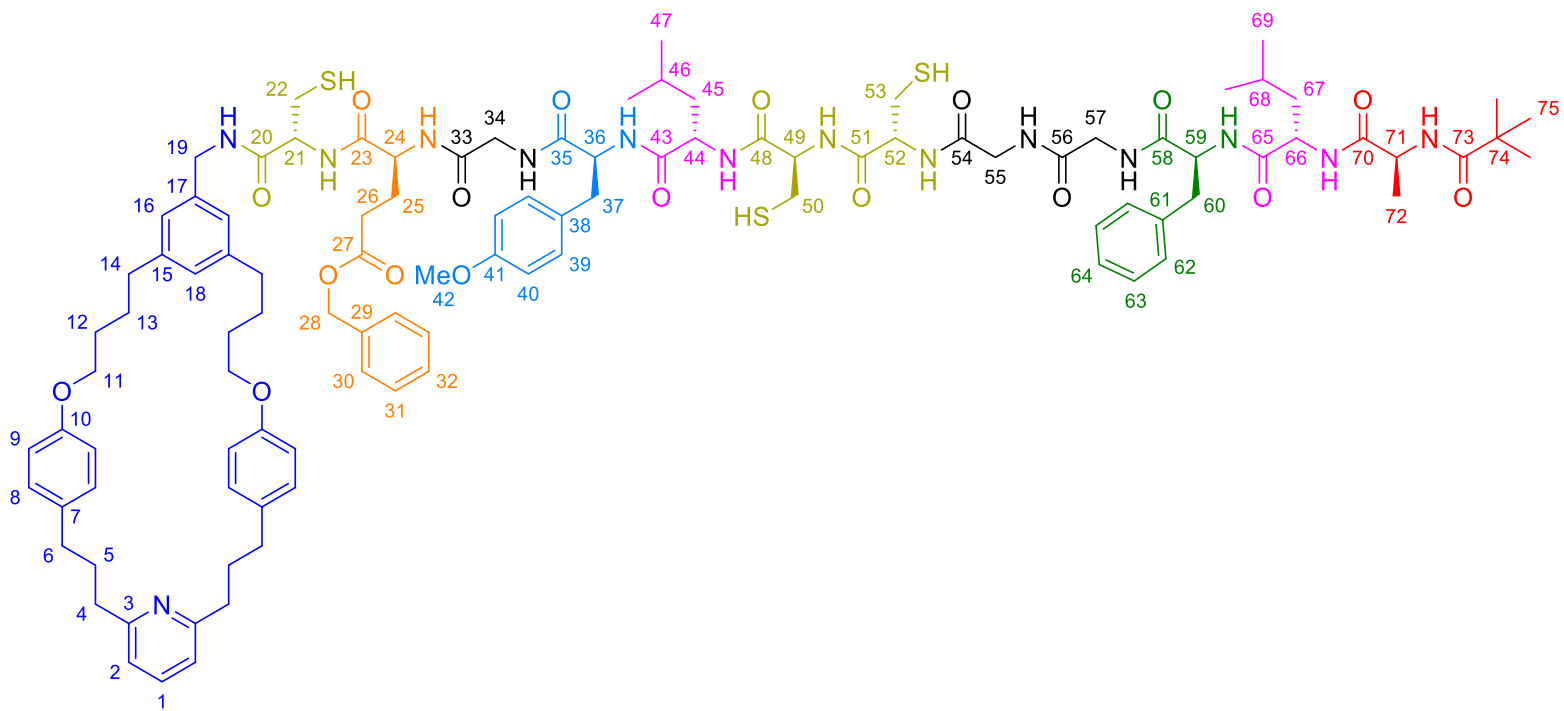

$7(8.0 \mathrm{mg}, 5.9 \mu \mathrm{mol})$ and $8(8.0 \mathrm{mg}, 6.6 \mu \mathrm{mol})$ were dissolved in $\mathrm{MeOH}(2 \mathrm{~mL})$ and $\mathrm{MeONH}_{2} \cdot \mathrm{HCl}$ (24 mg, $0.3 \mathrm{mmol}, 60$ equiv.) was added. The mixture was stirred at $50{ }^{\circ} \mathrm{C}$ for 3 $\mathrm{h}$. Then the solution was concentrated to dryness and left under high vacuum overnight. The crude was analysed using LRMS detecting the presence of unprotected products 9, which appeared as disulfide $\mathbf{S 1 7}$ (presumably formed during the time it took to analyse the sample), and $\mathbf{1 0}$ together with oxime S25 resulting from cleavage of the hydrazine (see Figures S10 and S11). The crude mixture was used in next step without any further purification.

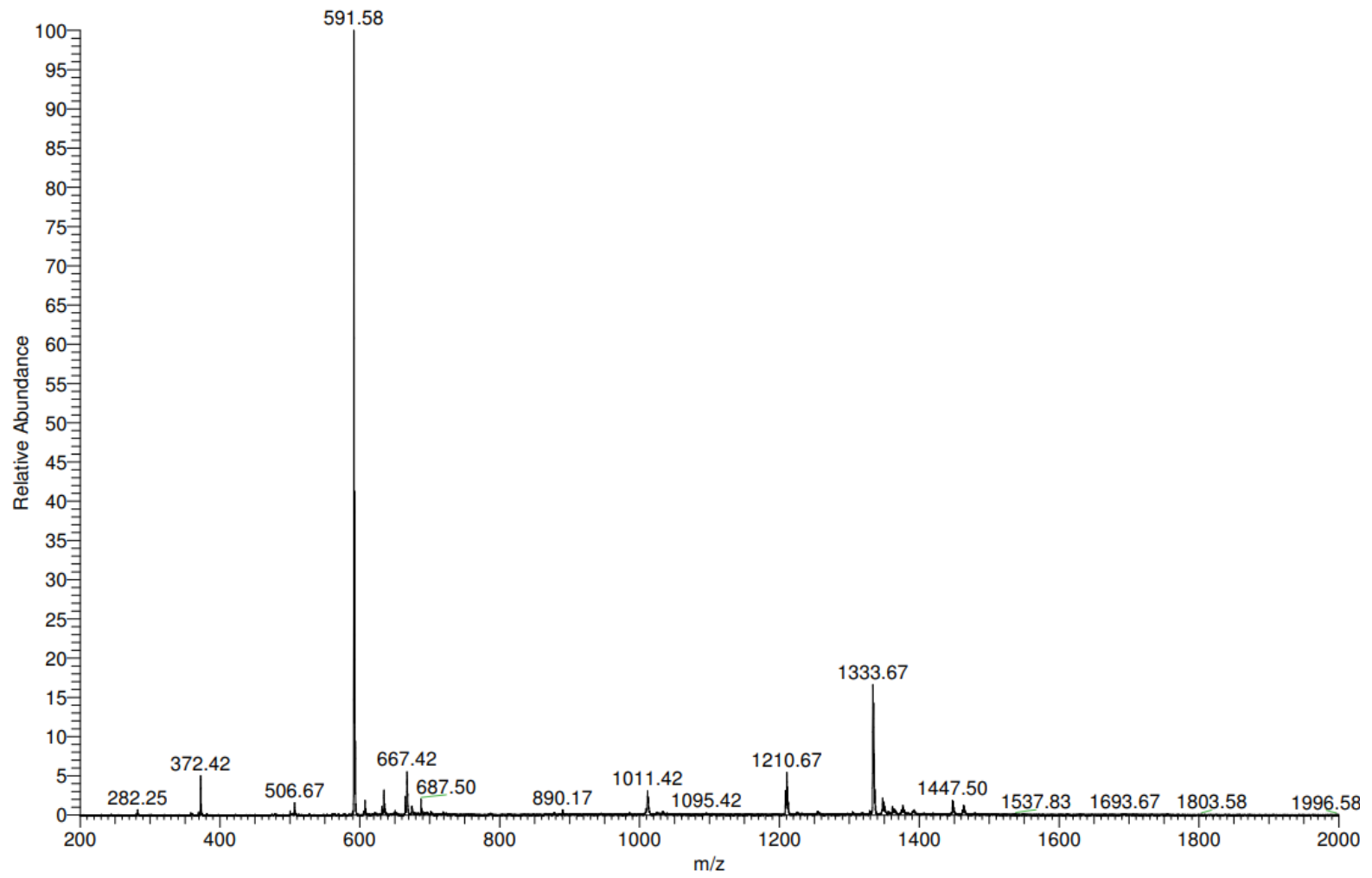

Figure S10. Mass spectrum $\left(\mathrm{ESI}^{+}\right)$of crude deprotection of 7 and 8. Peaks $(\mathrm{m} / \mathrm{z}): 591.58[\mathbf{S 2 5}+\mathrm{H}]^{+}$, $1333.67[\mathbf{S} 17+\mathrm{H}]^{+}$. 


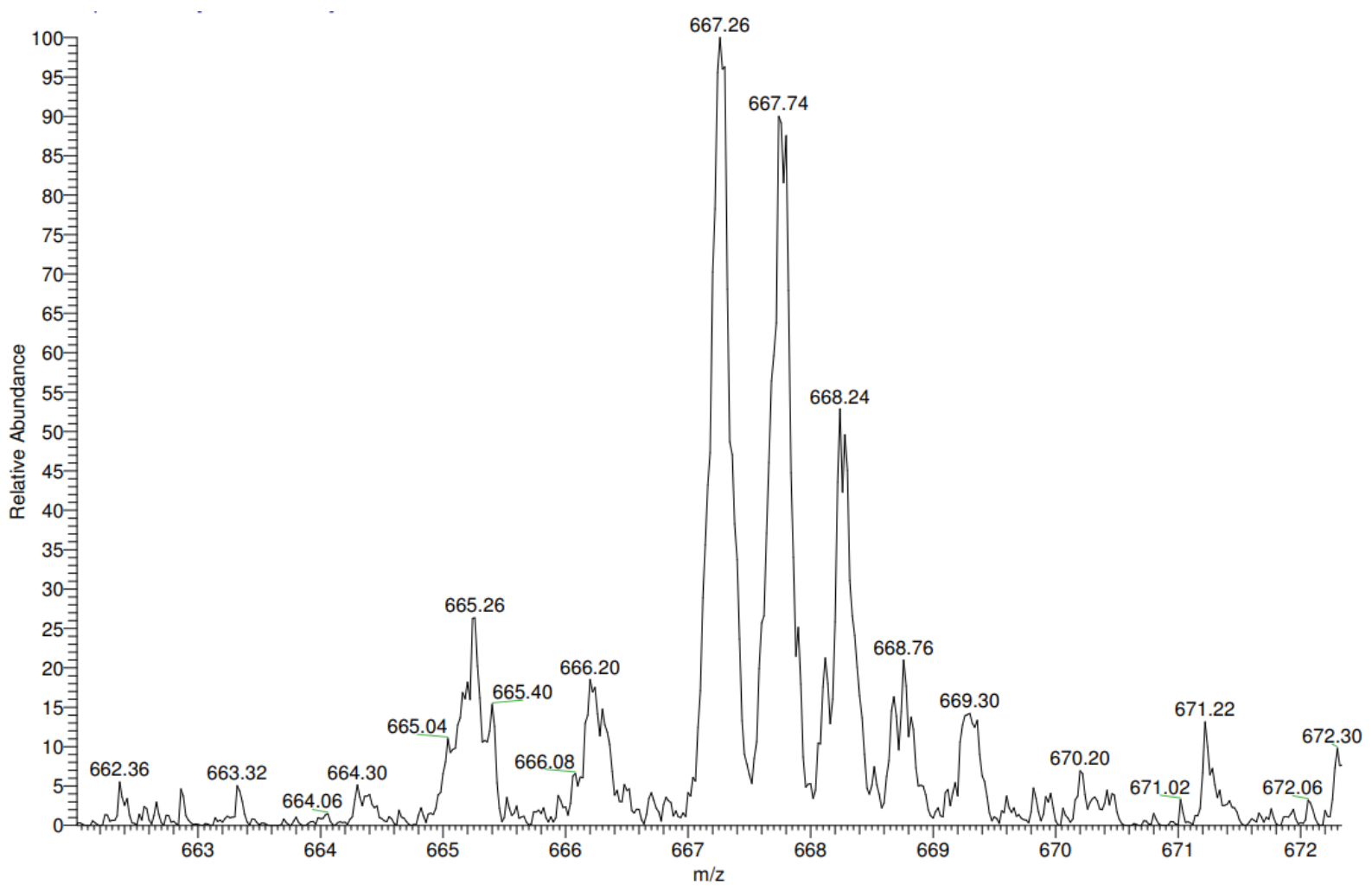

Figure S11. Mass spectrum $\left(E S I^{+}\right)$. Amplified region from m/z 662 to 673 . Peaks $(\mathrm{m} / \mathrm{z}): 665.26[10+\mathrm{H}]^{+}$, $667.26[\mathbf{S} 17+2 \mathrm{H}]^{2+}$.

The crude mixture containing $\mathbf{9}$ and $\mathbf{1 0}$ was dissolved in HFIP $(1.2 \mathrm{~mL})$ and ligation buffer [1.2 $\left.\mathrm{mL}, \mathrm{pH}=3,\left(6 \mathrm{M} \mathrm{Gn} \cdot \mathrm{HCl}, 0.2 \mathrm{M} \mathrm{Na}_{2} \mathrm{HPO}_{4}\right)\right]$. The solution was stirred vigorously and cooled to $-10{ }^{\circ} \mathrm{C}$. An aqueous solution of $\mathrm{NaNO}_{2}(0.2 \mathrm{M}, 0.24 \mathrm{~mL})$ was added dropwise, then $\mathrm{pH}$ was adjusted to $\mathrm{pH}=1$ with $1 \mathrm{M} \mathrm{HCl}$ and the solution was stirred at $-10{ }^{\circ} \mathrm{C}$ for $20 \mathrm{~min}$. A solution of 4-mercaptophenylacetic acid (MPAA) $0.2 \mathrm{M}$ in ligation buffer $\mathrm{pH}=7(6 \mathrm{M} \mathrm{Gn} \cdot \mathrm{HCl}, 0.2 \mathrm{M}$ $\left.\mathrm{Na}_{2} \mathrm{HPO}_{4}\right)(2.4 \mathrm{~mL})$ was added to the reaction mixture. The $\mathrm{pH}$ of the reaction was adjusted to $\mathrm{pH} 7$ by dropwise addition of $2 \mathrm{M} \mathrm{NaOH}$. The reaction was stirred at room temperature for $3 \mathrm{~h}$. Then TCEP.HCl (36 mg, 20 equiv.) was added and the solution was stirred for $20 \mathrm{~min}$. The HFIP layer was separated in centrifuge tube and the aqueous layer was diluted to $15 \mathrm{~mL}$ with $\mathrm{MeOH}$. After some minutes a precipitate appeared that was collected by centrifuge (10 min at $6000 \mathrm{rpm}$ ). The supernatant was decanted off and the residue was suspended in $\mathrm{MeOH}$ $(5 \mathrm{~mL})$, centrifuged $(10 \mathrm{~min}$ at $6000 \mathrm{rpm})$ and the supernatant discarded. The remaining solid was dried under high vacuum for $1 \mathrm{~h}$ to afford 11 as a colorless solid $(3 \mathrm{mg}, 1.5 \mu \mathrm{mol}, 25 \%$ yield).

${ }^{1} \mathrm{H}$ NMR $\left(600 \mathrm{MHz}, \mathrm{DMSO}-\mathrm{d}_{6}\right) \delta 8.46(\mathrm{~s}, 1 \mathrm{H}, \mathrm{NH}), 8.30-8.23(\mathrm{~m}, 2 \mathrm{H}, \mathrm{NH}), 8.21-8.17(\mathrm{~m}$, $2 \mathrm{H}, \mathrm{NH}), 8.10(\mathrm{~d}, J=6.9 \mathrm{~Hz}, 1 \mathrm{H}, \mathrm{NH}), 8.08-7.92(\mathrm{~m}, 4 \mathrm{H}, \mathrm{NH}), 7.91-7.87(\mathrm{~m}, 1 \mathrm{H}, \mathrm{NH}), 7.67$ $(\mathrm{d}, J=7.9 \mathrm{~Hz}, 1 \mathrm{H}, \mathrm{NH}), 7.58\left(\mathrm{t}, J=7.7 \mathrm{~Hz}, 1 \mathrm{H}, \mathrm{H}_{1}\right), 7.42(\mathrm{~d}, J=7.5 \mathrm{~Hz}, 1 \mathrm{H}, \mathrm{NH}), 7.36-7.27$ 
$\left(m, 5 \mathrm{H}, \mathrm{H}_{30}+\mathrm{H}_{31}+\mathrm{H}_{32}\right), 7.25-7.14\left(\mathrm{~m}, 5 \mathrm{H}, \mathrm{H}_{62}+\mathrm{H}_{63}+\mathrm{H}_{64}\right), 7.12\left(\mathrm{~d}, J=7.9 \mathrm{~Hz}, 2 \mathrm{H}, \mathrm{H}_{39}\right)$, $7.06\left(\mathrm{~d}, J=7.6 \mathrm{~Hz}, 2 \mathrm{H}, \mathrm{H}_{2}\right), 7.02\left(\mathrm{~d}, J=8.0 \mathrm{~Hz}, 4 \mathrm{H}, \mathrm{H}_{8}\right), 6.93\left(\mathrm{~s}, 1 \mathrm{H}, \mathrm{H}_{18}\right), 6.90\left(\mathrm{~s}, 2 \mathrm{H}, \mathrm{H}_{16}\right)$, $6.78\left(\mathrm{~d}, J=8.2 \mathrm{~Hz}, 2 \mathrm{H}, \mathrm{H}_{40}\right), 6.72\left(\mathrm{~d}, \mathrm{~J}=8.1 \mathrm{~Hz}, 4 \mathrm{H}, \mathrm{H}_{9}\right), 5.07\left(\mathrm{~s}, 2 \mathrm{H}, \mathrm{H}_{28}\right), 4.55-4.33(\mathrm{~m}$, $\left.6 \mathrm{H}, \mathrm{H}_{59}+\mathrm{H}_{24}+\mathrm{H}_{36}+\mathrm{H}_{49}+\mathrm{H}_{21}+\mathrm{H}_{52}\right), 4.32-4.18\left(\mathrm{~m}, 5 \mathrm{H}, \mathrm{H}_{71}+\mathrm{H}_{19}+\mathrm{H}_{44}+\mathrm{H}_{66}\right), 3.87(\mathrm{t}, \mathrm{J}=$ $\left.5.8 \mathrm{~Hz}, 4 \mathrm{H}, \mathrm{H}_{11}\right), 3.80-3.71\left(\mathrm{~m}, 6 \mathrm{H}, \mathrm{H} 34, \mathrm{H}_{55}+\mathrm{H}_{57}\right), 3.69\left(\mathrm{~s}, 3 \mathrm{H}, \mathrm{H}_{42}\right), 3.05$ (dd, J = 14.4, 4.6 $\left.\mathrm{Hz}, 1 \mathrm{H}, \mathrm{H}_{60}\right), 2.95\left(\mathrm{~d}, J=11.3 \mathrm{~Hz}, 1 \mathrm{H}, \mathrm{H}_{37}\right), 2.86-2.71\left(\mathrm{~m}, 6 \mathrm{H}, \mathrm{H}_{60^{\prime}}+\mathrm{H}_{37}+\mathrm{H}_{53}+\mathrm{H}_{50}\right), 2.68$ $\left(\mathrm{d}, J=7.6 \mathrm{~Hz}, 4 \mathrm{H}, \mathrm{H}_{4}\right), 2.62-2.43\left(\mathrm{~m}, 8 \mathrm{H}, \mathrm{H}_{6}+\mathrm{H}_{14}\right), 2.43-2.21\left(\mathrm{~m}, 7 \mathrm{H}, \mathrm{H}_{25}+\mathrm{H}_{26}+\right.$ three $\mathrm{SH}), 2.05-1.96\left(\mathrm{~m}, 1 \mathrm{H}, \mathrm{H}_{22}\right), 1.96-1.87\left(\mathrm{~m}, 4 \mathrm{H}, \mathrm{H}_{5}\right), 1.87-1.79\left(\mathrm{~m}, 1 \mathrm{H}, \mathrm{H}_{22}\right), 1.71-1.62$ $\left(\mathrm{m}, 8 \mathrm{H}, \mathrm{H}_{12}+\mathrm{H}_{13}\right), 1.54-1.46\left(\mathrm{~m}, 2 \mathrm{H}, \mathrm{H}_{46}+\mathrm{H}_{68}\right), 1.40-1.31\left(\mathrm{~m}, 4 \mathrm{H}, \mathrm{H}_{45}+\mathrm{H}_{67}\right), 1.17(\mathrm{~d}, \mathrm{~J}=$ $7.2 \mathrm{~Hz}, 3 \mathrm{H}, \mathrm{H}_{72}$ ), $1.08\left(\mathrm{~s}, 9 \mathrm{H}, \mathrm{H}_{75}\right), 0.85-0.75\left(\mathrm{~m}, 12 \mathrm{H}, \mathrm{H}_{47}+\mathrm{H}_{69}\right.$ ). 2D NMR techniques (COSY, $\mathrm{HSQC}, \mathrm{HMBC}$ ) were used to aid in the assignment of this compound; ${ }^{13} \mathrm{C}$ NMR $(151 \mathrm{MHz}$, DMSO- $\left.d_{6}\right) \delta 177.29,172.27,171.79,171.65,171.32,170.92,169.72,169.44,169.03,168.78$, $160.54,157.76,156.75,141.83,138.91,137.64,136.64,136.14,133.93,130.17,130.04$, $129.43,129.14,128.40,128.35,128.01,127.97,127.88,127.74,126.66,126.22,124.78$, $120.15,114.09,113.49,113.42,67.03,65.48,65.38,55.23,54.96,54.89,53.97,53.63,51.77$, $51.07,48.60,48.17,42.12,41.95,40.88,40.55,37.90,37.32,36.65,36.55,34.36,33.67$, $31.36,30.00,27.87,27.55,27.32,27.21,26.56,26.38,26.01,23.97,23.04,23.01,22.95$, 21.69, 21.55, 21.48, 17.46; HRMS $\left(\mathrm{ESI}^{+}\right)$Calculated for $\mathrm{C}_{104} \mathrm{H}_{139} \mathrm{O}_{18} \mathrm{~N}_{14} \mathrm{~S}_{3}: 1967.9543[\mathrm{M}+\mathrm{H}]^{+}$, found 1967.9512 . 


\subsection{Glutamic acid cleavage of compound 11}

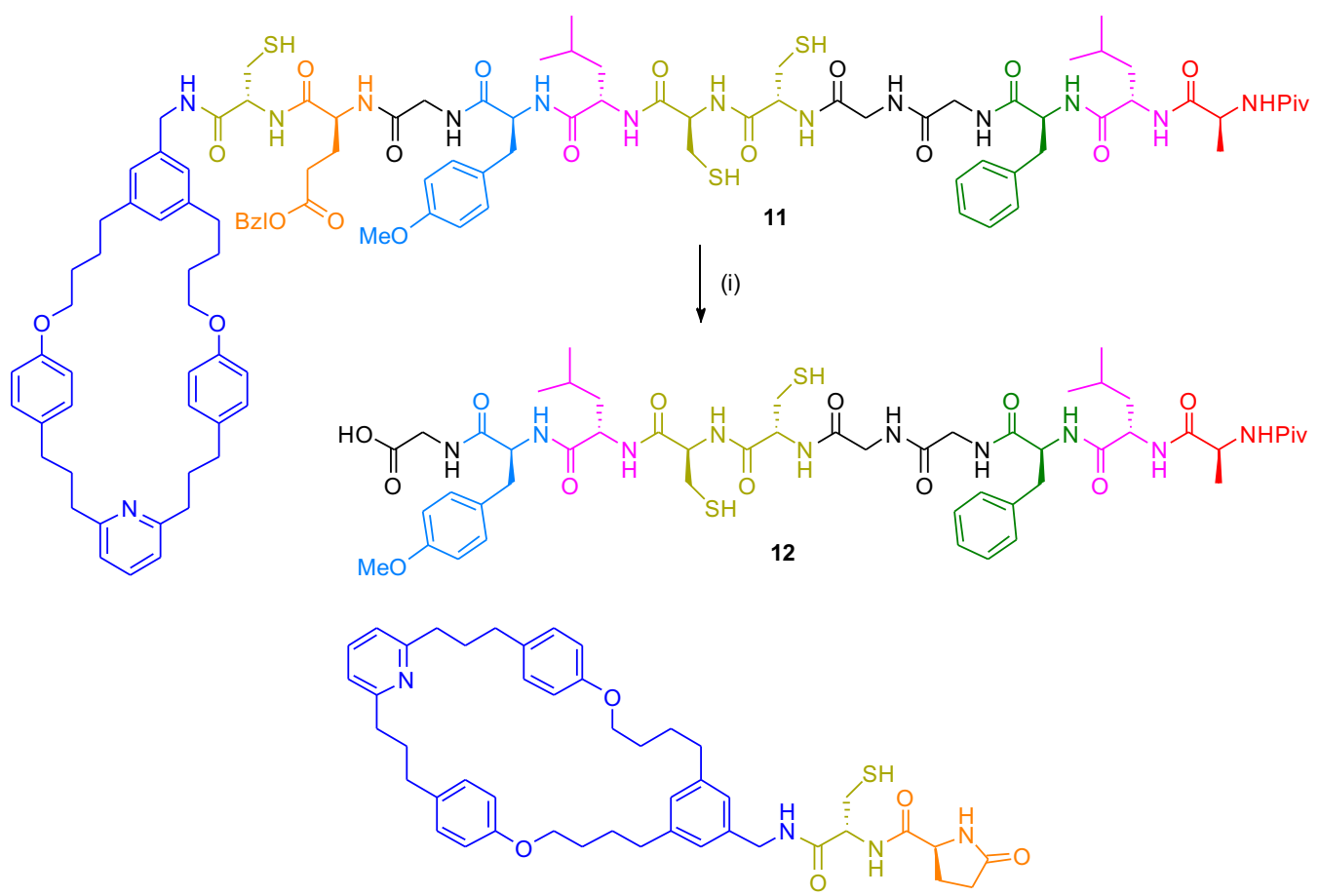

13

Scheme S14. Cleavage of peptide 11 to release acid 12 and lactam 13. Reagents and conditions: (i) $\mathrm{LiOH}, \mathrm{THF}: \mathrm{H}_{2} \mathrm{O}(10: 1)$, rt, $30 \mathrm{~min}$.

Peptide 11 (0.50 mg, $0.25 \mu \mathrm{mol}, 1.0$ equiv.) was dissolved in THF (1.0 mL) and $\mathrm{H}_{2} \mathrm{O}(0.1 \mathrm{~mL})$. Then $\mathrm{LiOH}(0.06 \mathrm{mg} 2.5 \mu \mathrm{mol}, 10$ equiv.) was added. The mixture was allowed to stir for 30 min at room temperature. Then the mixture was extracted with a mixture of $i \mathrm{PrOH}: \mathrm{CHCl}_{3}(1: 3)$ twice. The organic phase was washed with water, dried over $\mathrm{MgSO}_{4}$. The crude was directly analysed by mass spectrometry (figures $\mathrm{S} 12$ and $\mathrm{S} 13$ ):

LRMS $\left(\mathrm{ESI}^{+}\right) 777.67[13+\mathrm{H}]^{+}, 1107.67[12+\mathrm{Li}]^{+}$.

LRMS (ESI') $1100.92\left[12-\mathrm{H}^{-}, 1136.25\right.$ [12+CI] 
SI Echavarren et al, 'Sequence-Selective Decapeptide Synthesis by the Parallel Operation of ...'

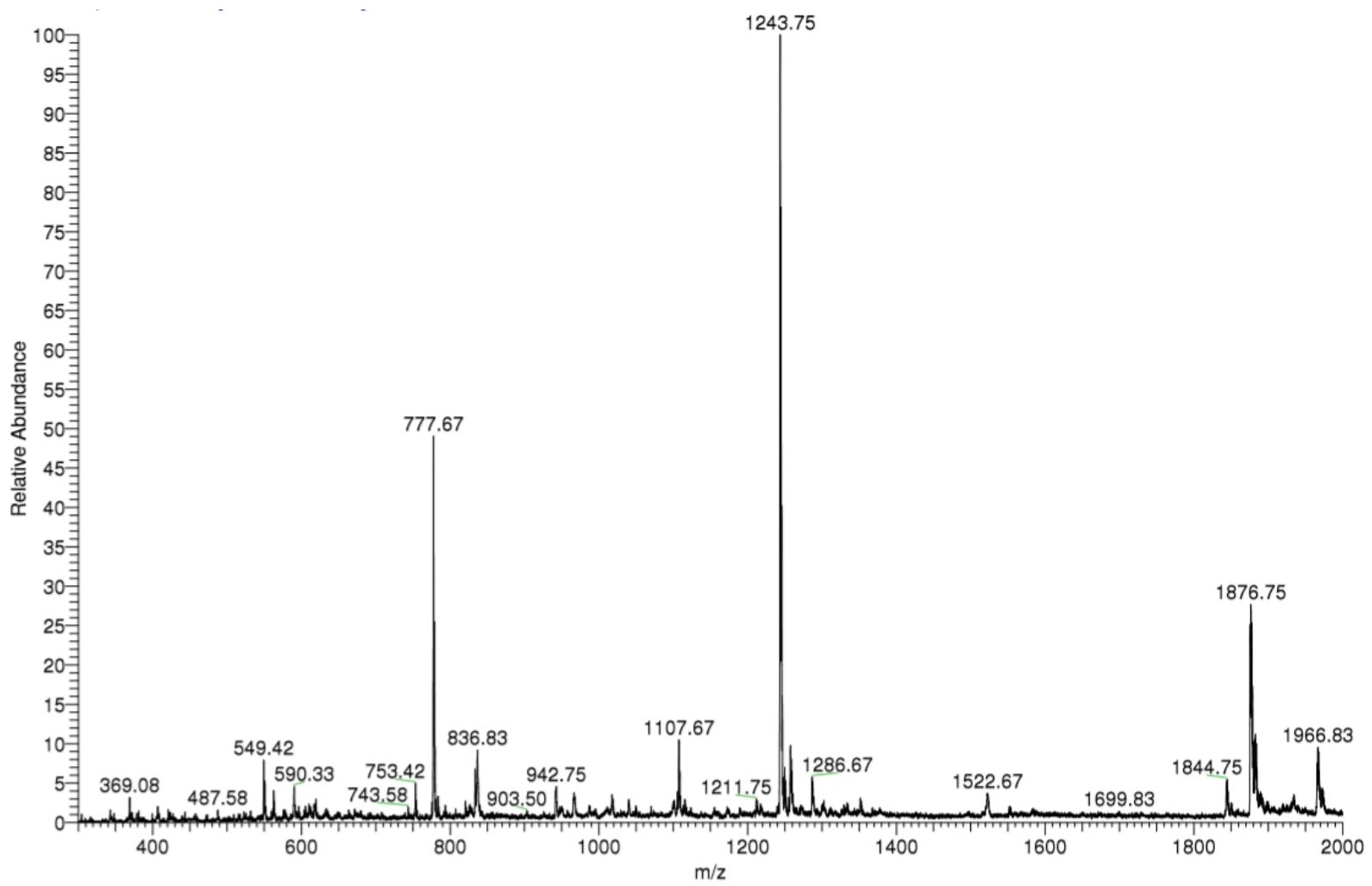

Figure S12. Mass spectrum $\left(\mathrm{ESI}^{+}\right)$of crude reaction of hydrolysis and Glu cleavage of substrate 11. Peaks (m/z): $777.67[13+\mathrm{H}]^{+}, 1107.67[12+\mathrm{Li}]^{+}$.

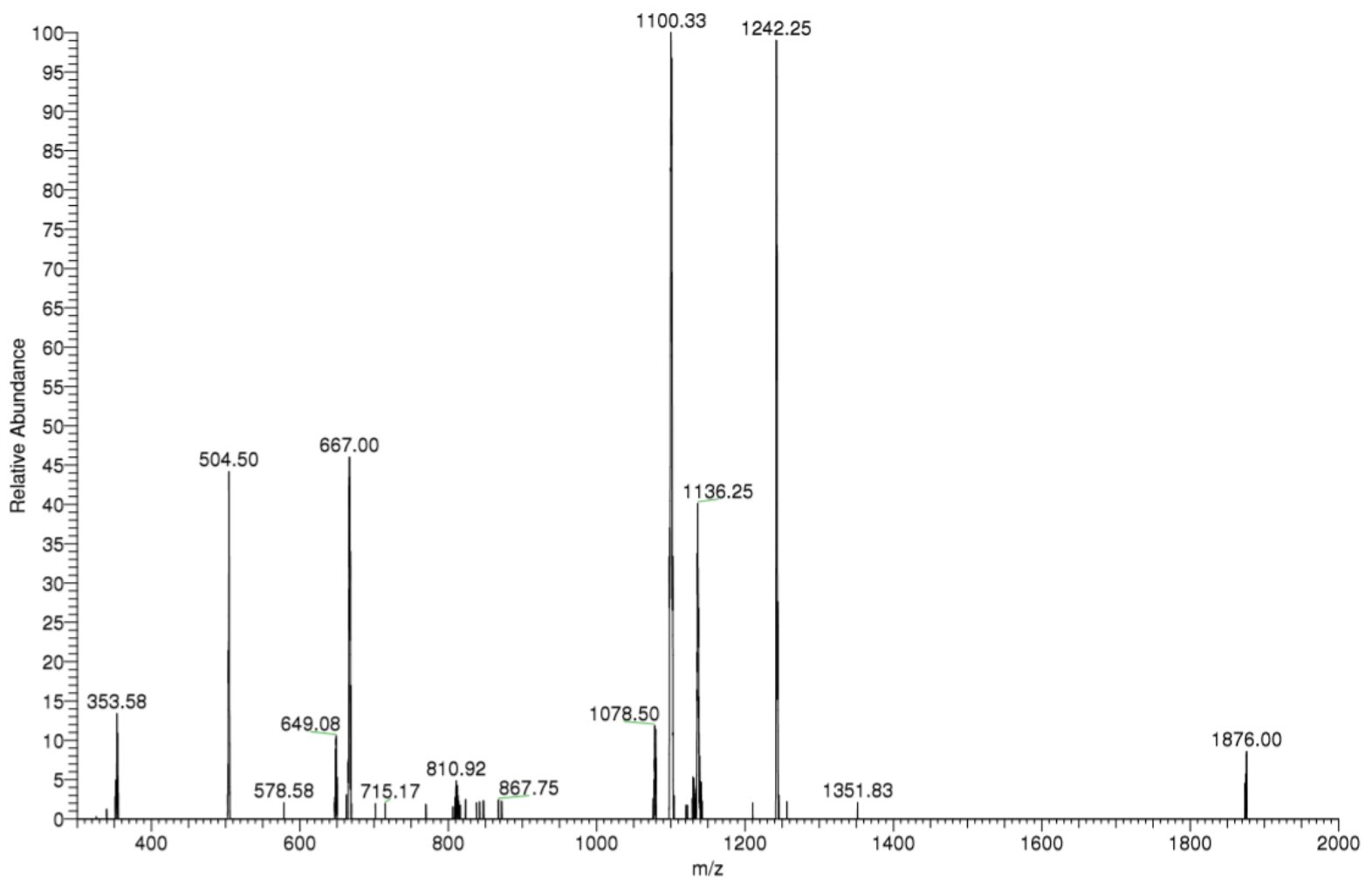

Figure S13. Mass spectrum (ESl') of crude reaction of hydrolysis and Glu cleavage of substrate 11. Peaks (m/z): $1100.92\left[12-\mathrm{H}^{-}, 1136.25[12+\mathrm{Cl}]^{-}\right.$. 
SI Echavarren et al, 'Sequence-Selective Decapeptide Synthesis by the Parallel Operation of ...'

\section{NMR and MS spectra}
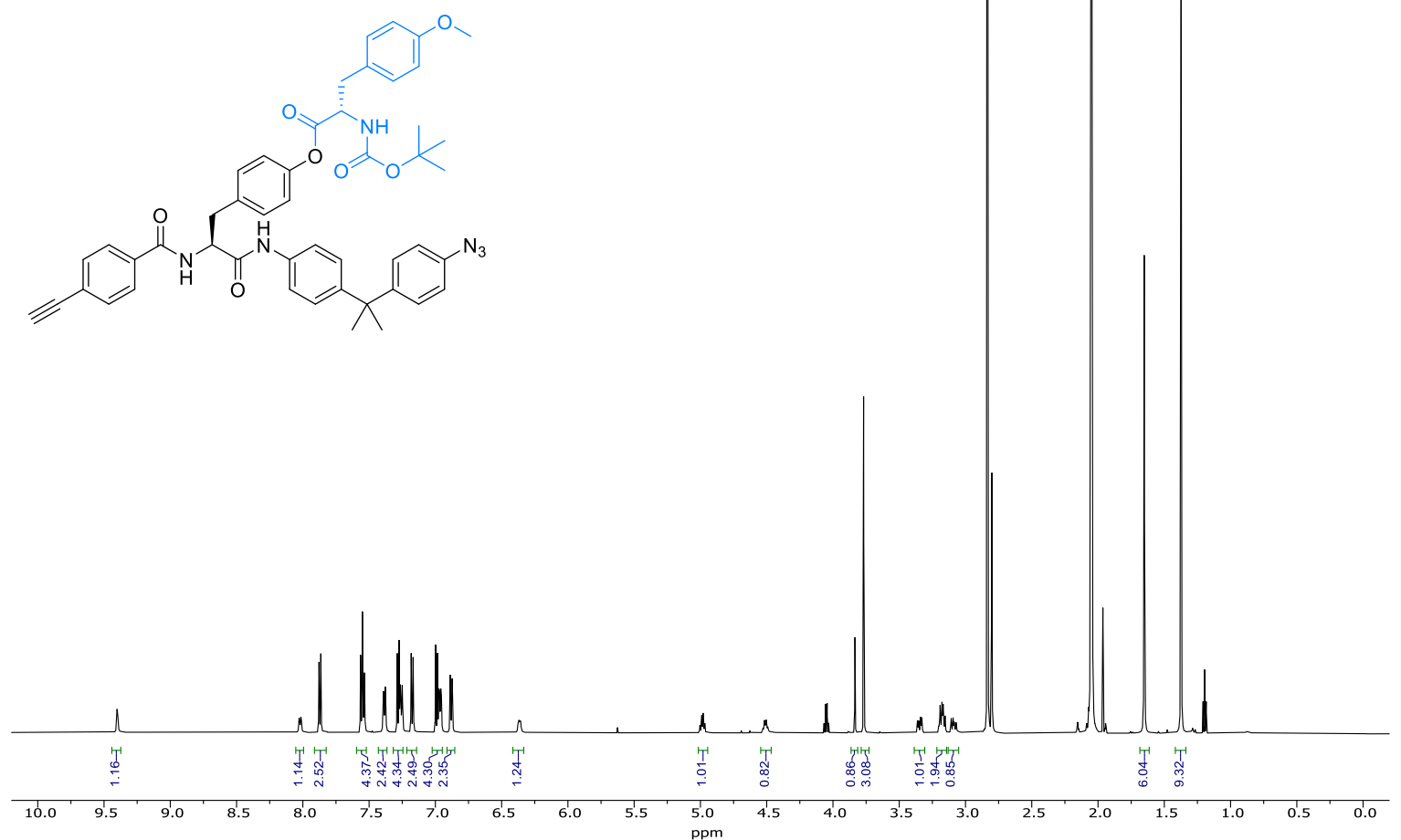

Spectrum 1. ${ }^{1} \mathrm{H}$ NMR $\left(600 \mathrm{MHz}\right.$, Acetone- $\left.d_{6}\right)$ of $\mathbf{S 2}$.

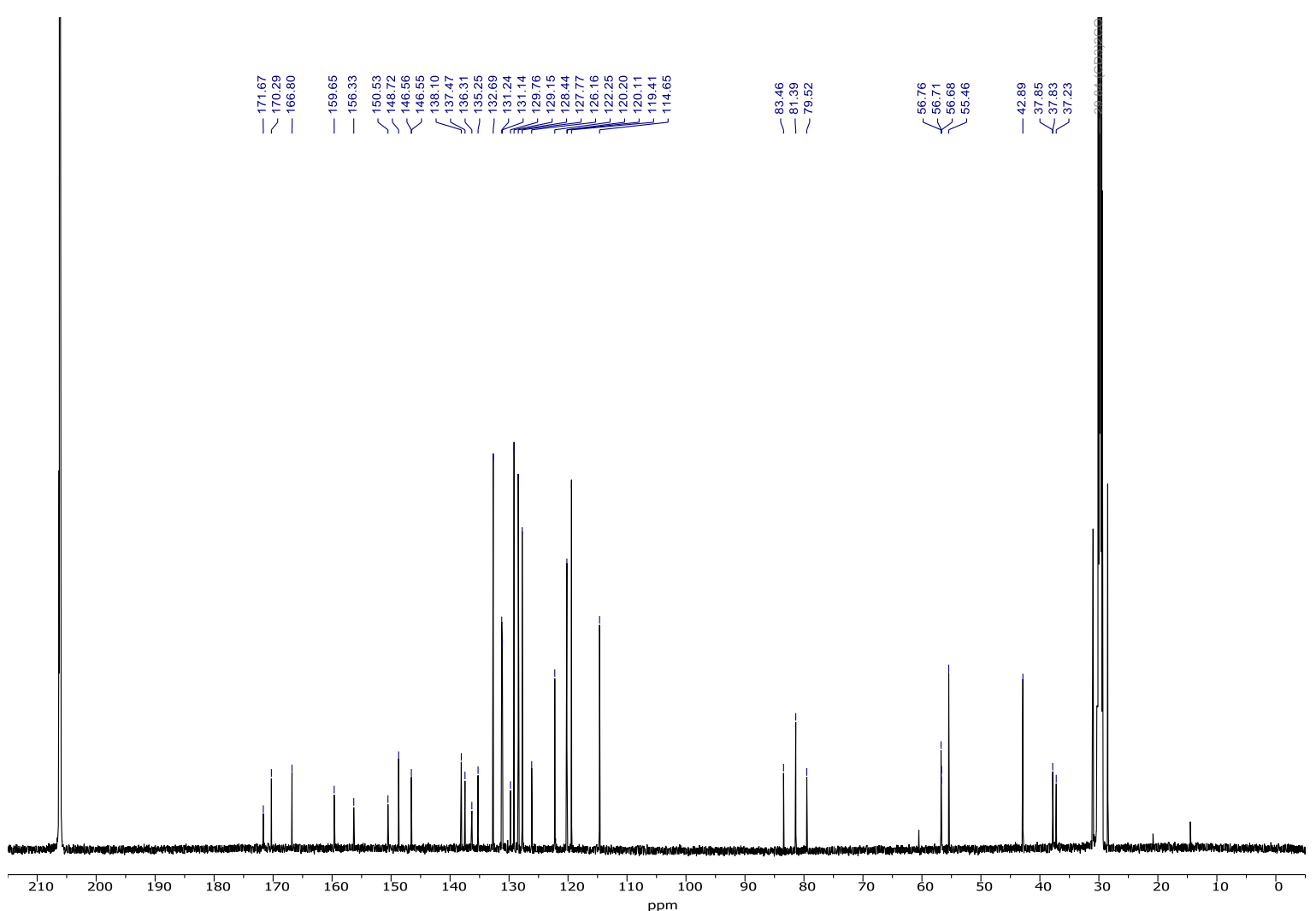

Spectrum 2. ${ }^{13} \mathrm{C}$ NMR $\left(151 \mathrm{MHz}\right.$, Acetone- $\left.d_{6}\right)$ of $\mathbf{S 2}$. 
SI Echavarren et al, 'Sequence-Selective Decapeptide Synthesis by the Parallel Operation of ...'
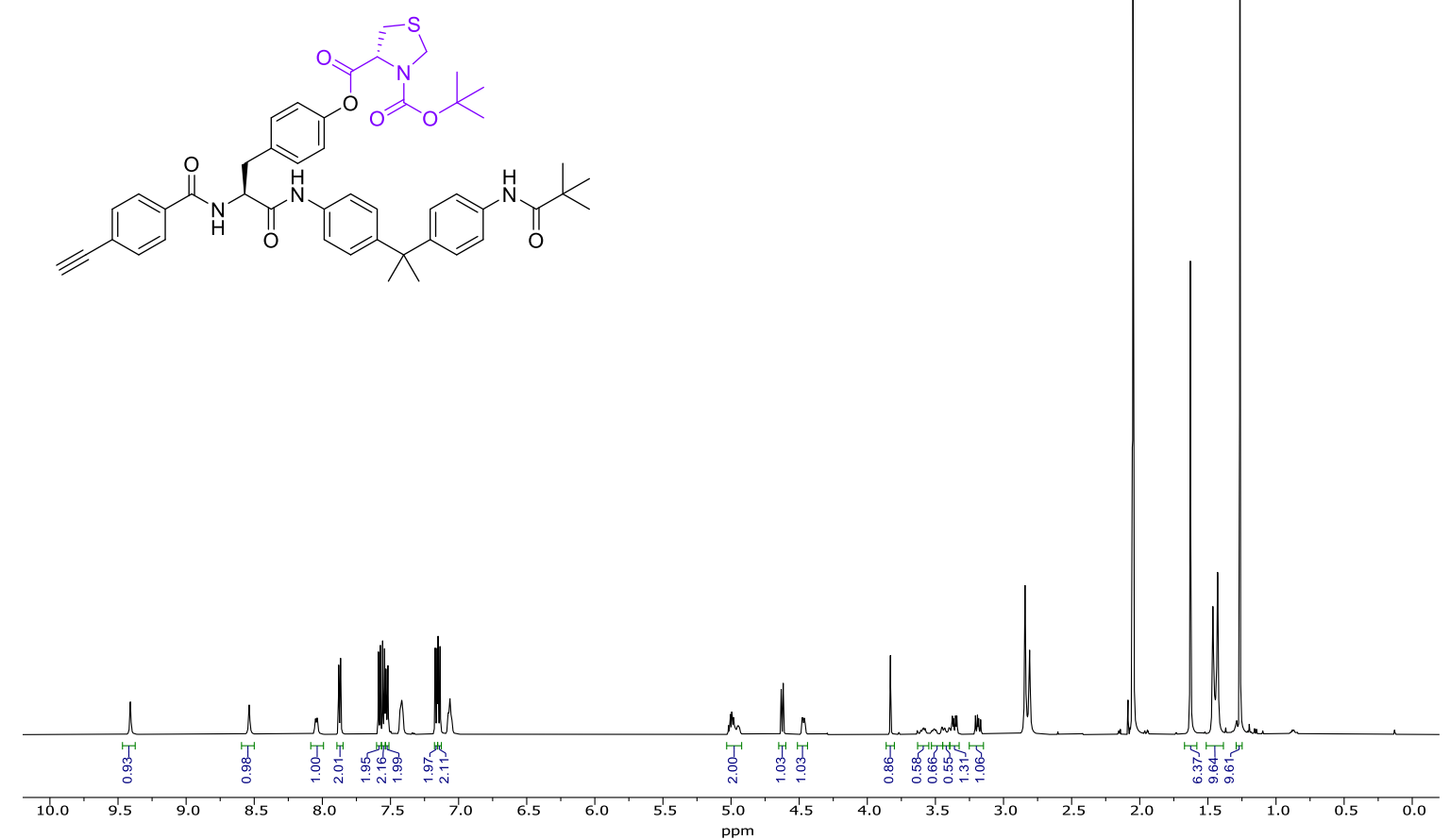

Spectrum 3. ${ }^{1} \mathrm{H}$ NMR $\left(600 \mathrm{MHz}\right.$, Acetone- $\left.d_{6}\right)$ of $\mathbf{S 4}$.

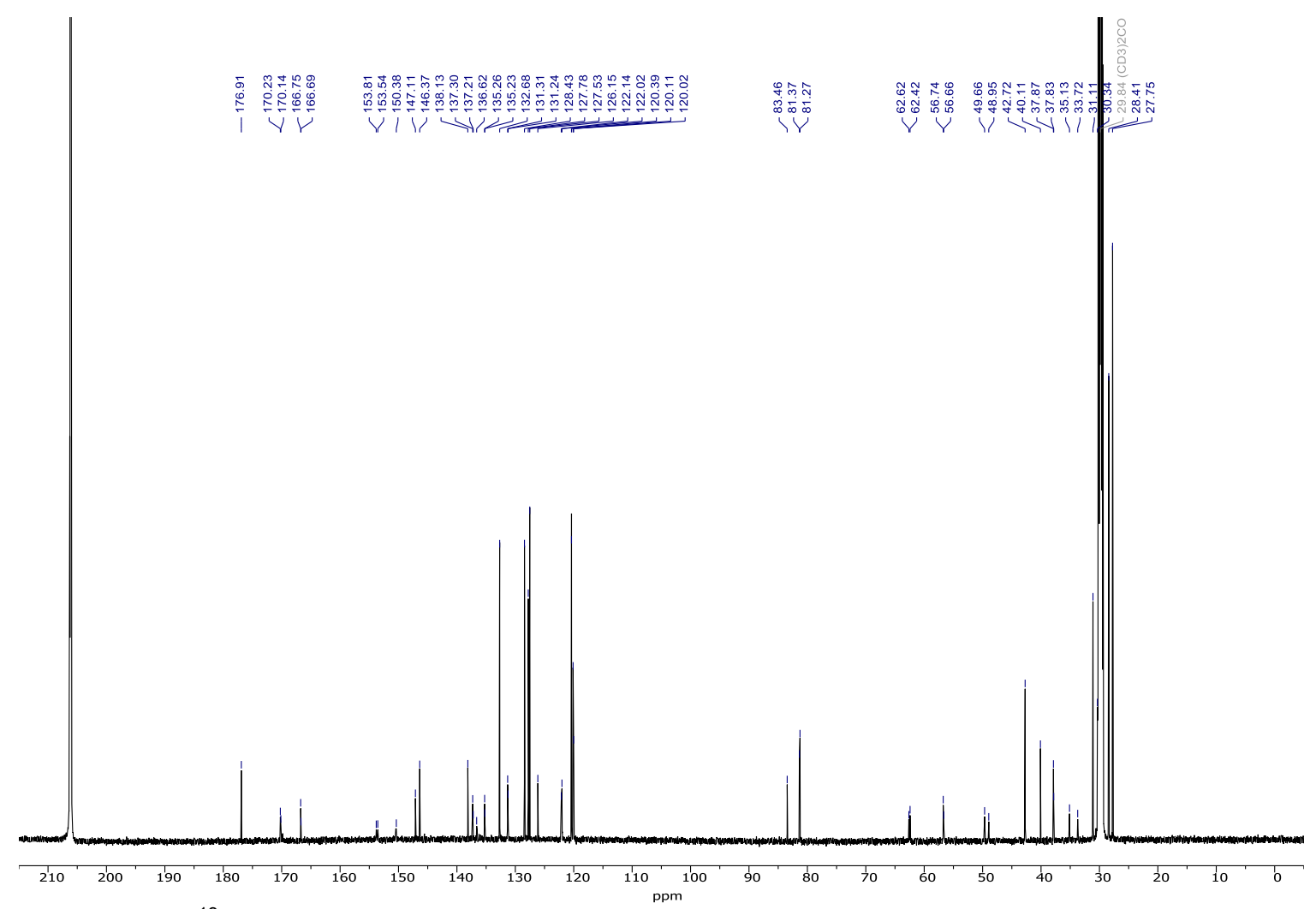

Spectrum 4. ${ }^{13} \mathrm{C}$ NMR $\left(151 \mathrm{MHz}\right.$, Acetone- $\left.d_{6}\right)$ of $\mathbf{S 4}$. 
SI Echavarren et al, 'Sequence-Selective Decapeptide Synthesis by the Parallel Operation of ...'

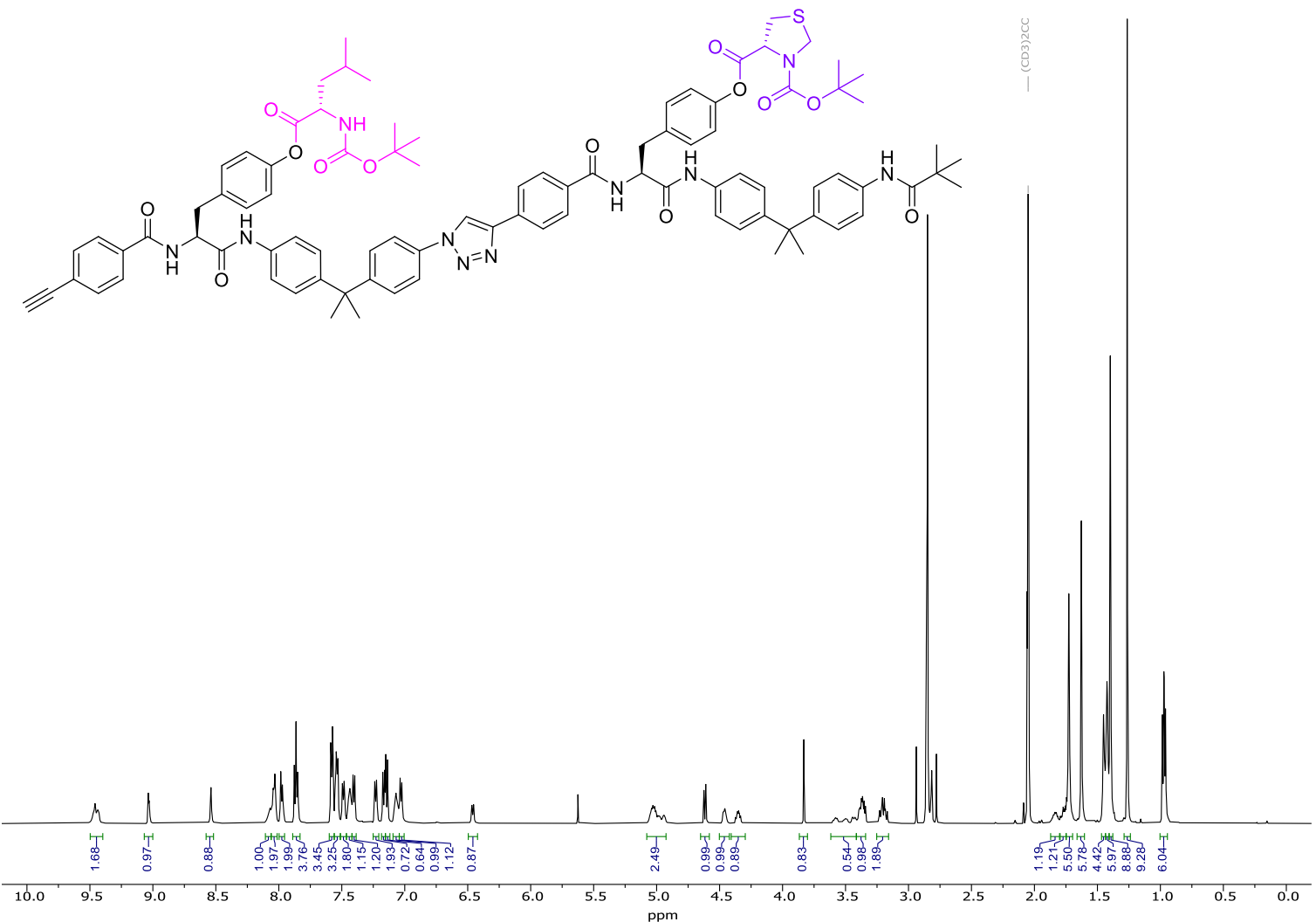

Spectrum 5. ${ }^{1} \mathrm{H}$ NMR $\left(600 \mathrm{MHz}\right.$, Acetone- $\left.d_{6}\right)$ of $\mathbf{5}$.

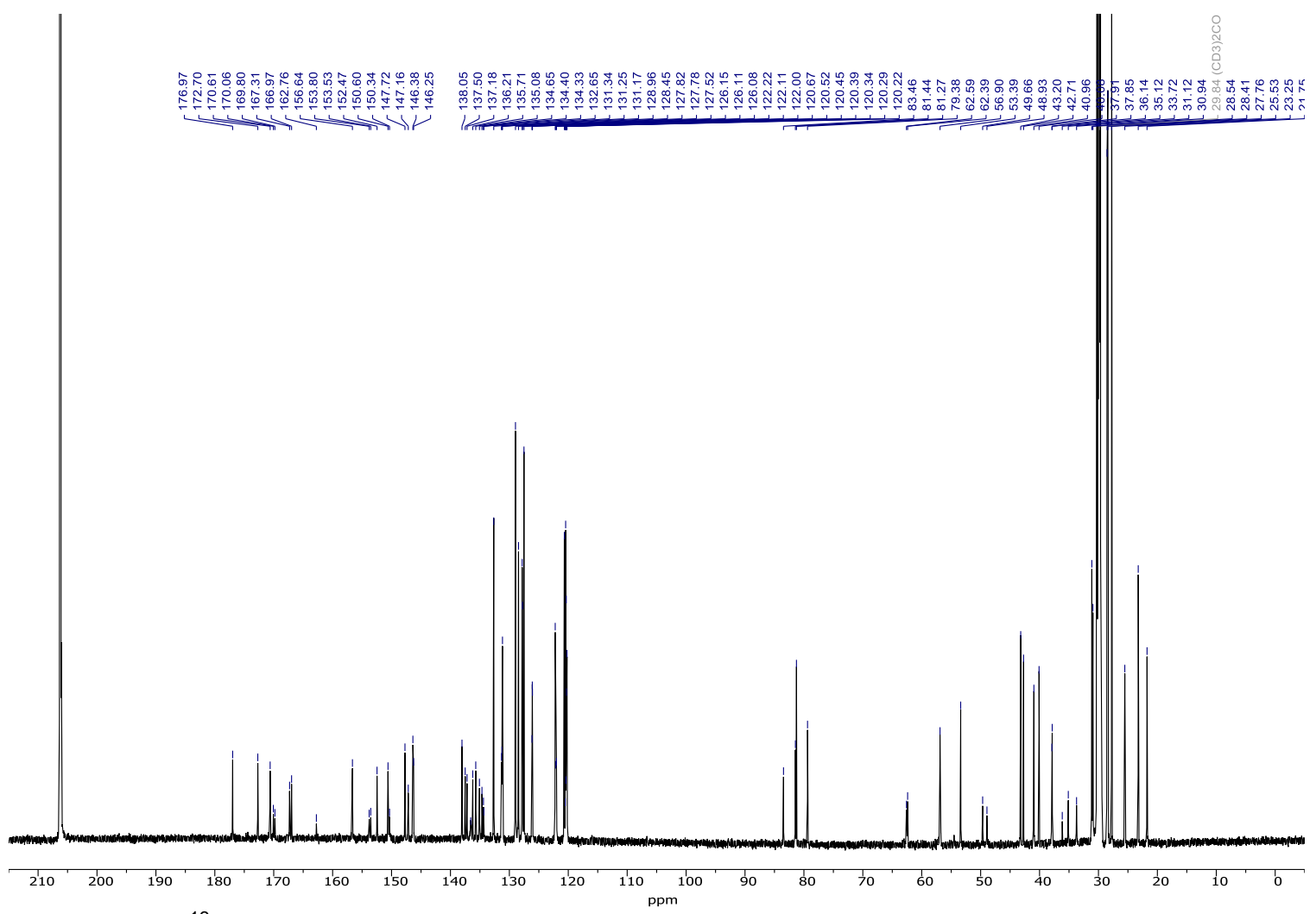

Spectrum 6. ${ }^{13} \mathrm{C}$ NMR $\left(151 \mathrm{MHz}\right.$, Acetone- $\left.d_{6}\right)$ of 5 . 
SI Echavarren et al, 'Sequence-Selective Decapeptide Synthesis by the Parallel Operation of ...'

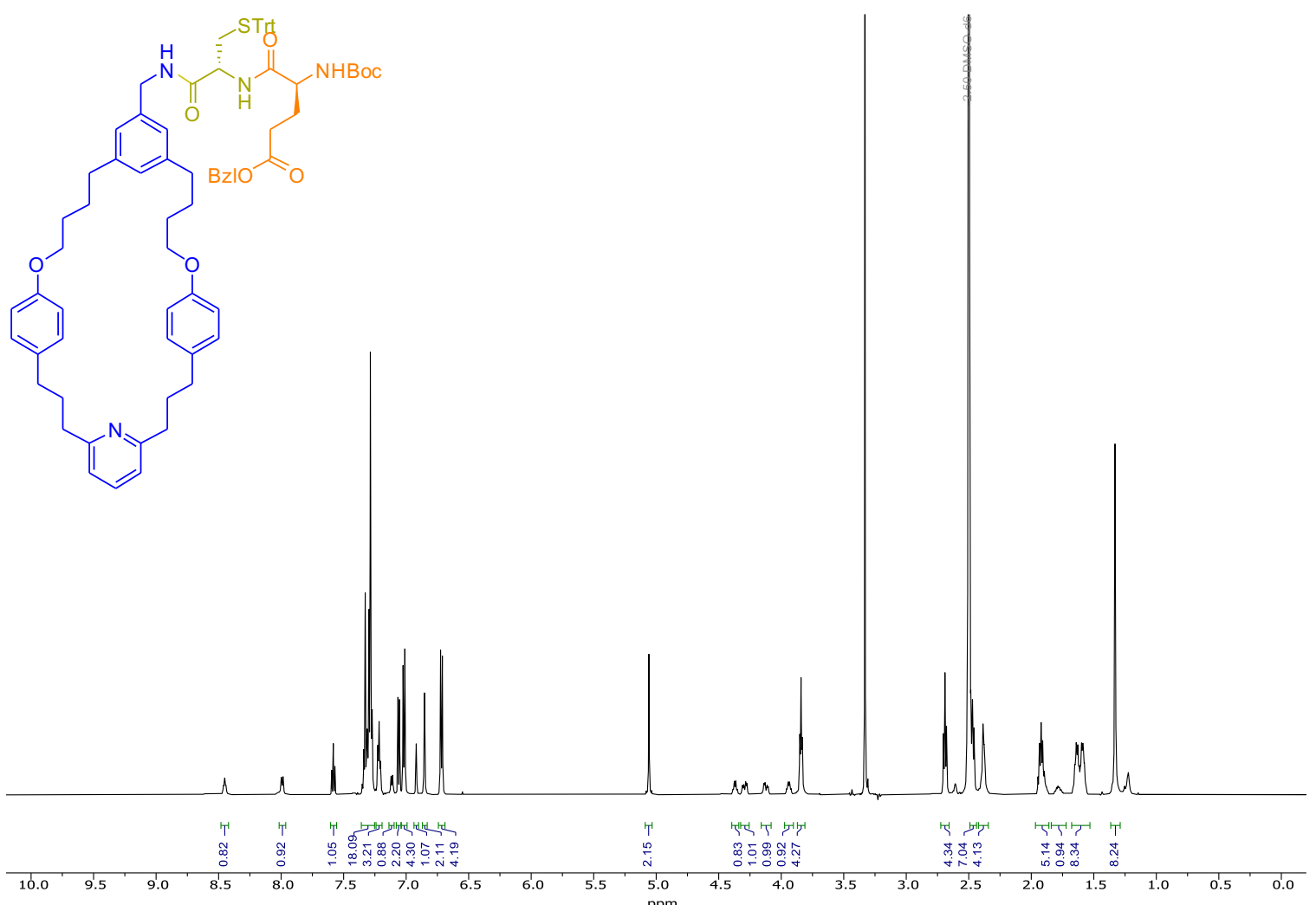

Spectrum 7. ${ }^{1} \mathrm{H}$ NMR $\left(600 \mathrm{MHz}\right.$, DMSO- $\left.d_{6}\right)$ of $\mathbf{S 7}$.

추웅

Nis

i) iा
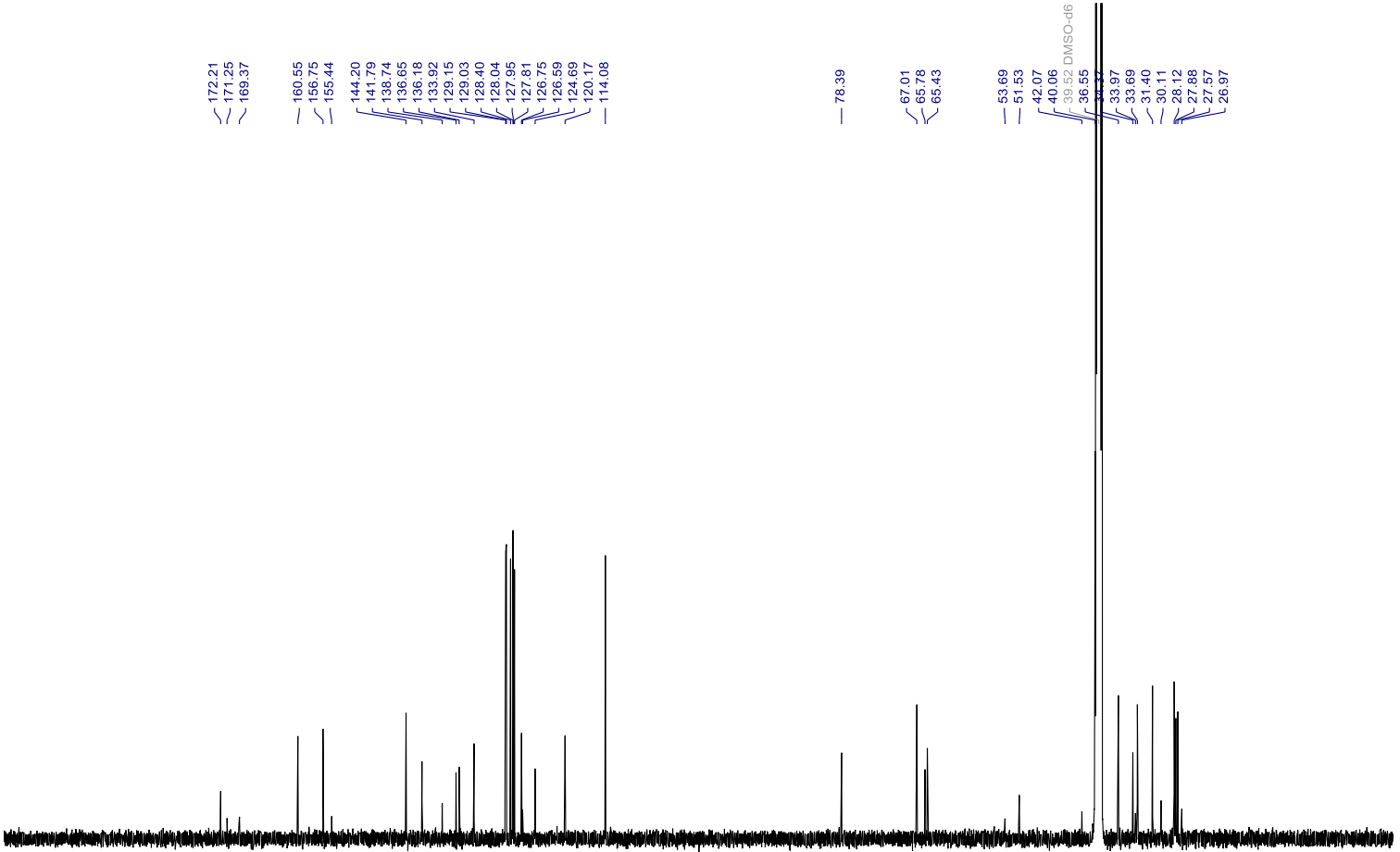

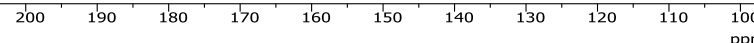

Spectrum 8. ${ }^{13} \mathrm{C}$ NMR $\left(151 \mathrm{MHz}\right.$, DMSO- $\left.d_{6}\right)$ of $\mathbf{S 7}$. 
SI Echavarren et al, 'Sequence-Selective Decapeptide Synthesis by the Parallel Operation of ...'

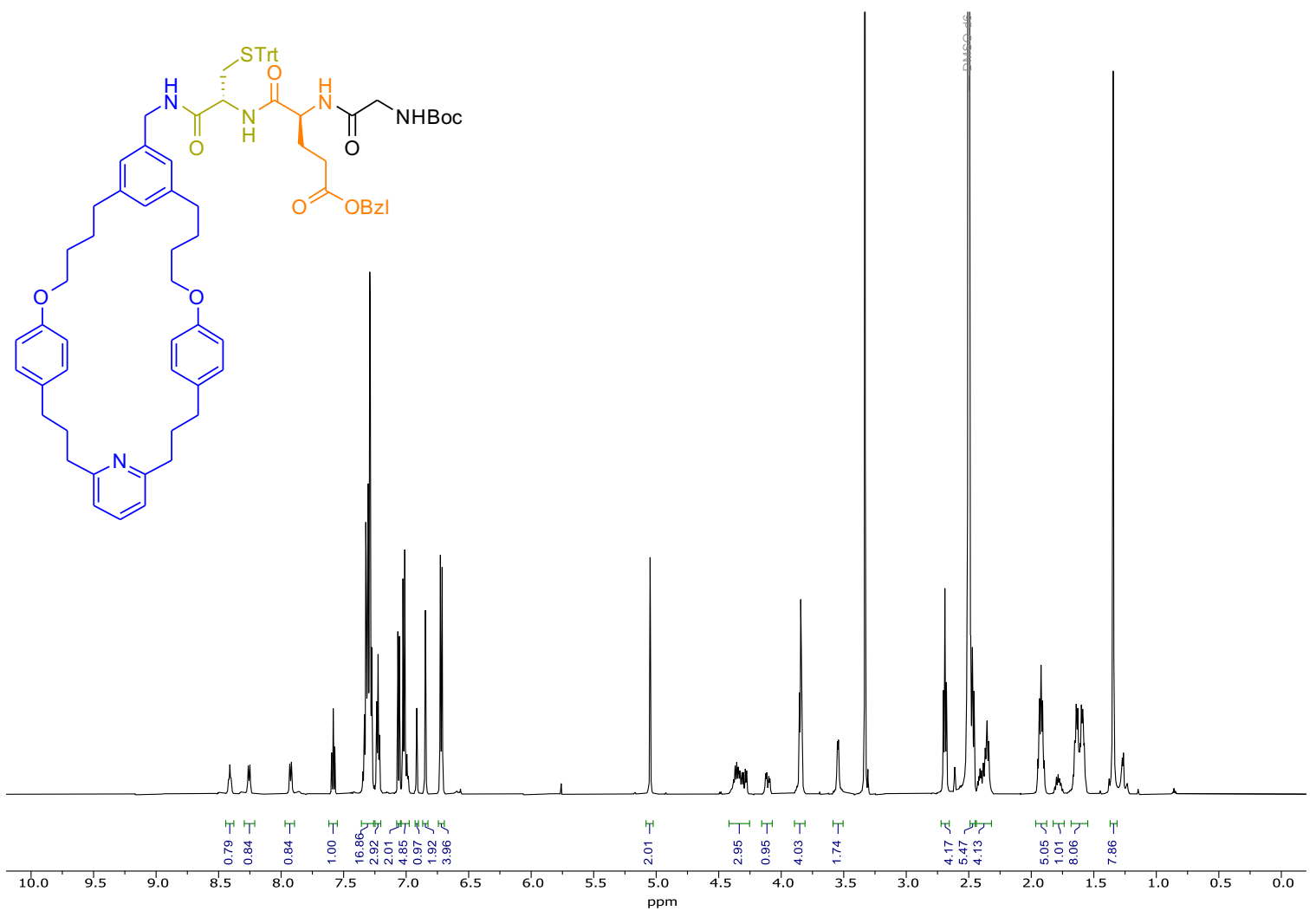

Spectrum 9. ${ }^{1} \mathrm{H}$ NMR $\left(600 \mathrm{MHz}, \mathrm{DMSO}-d_{6}\right)$ of $\mathbf{S 8}$.

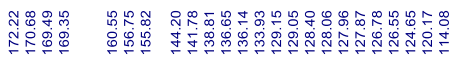

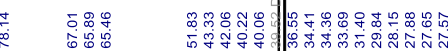

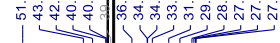

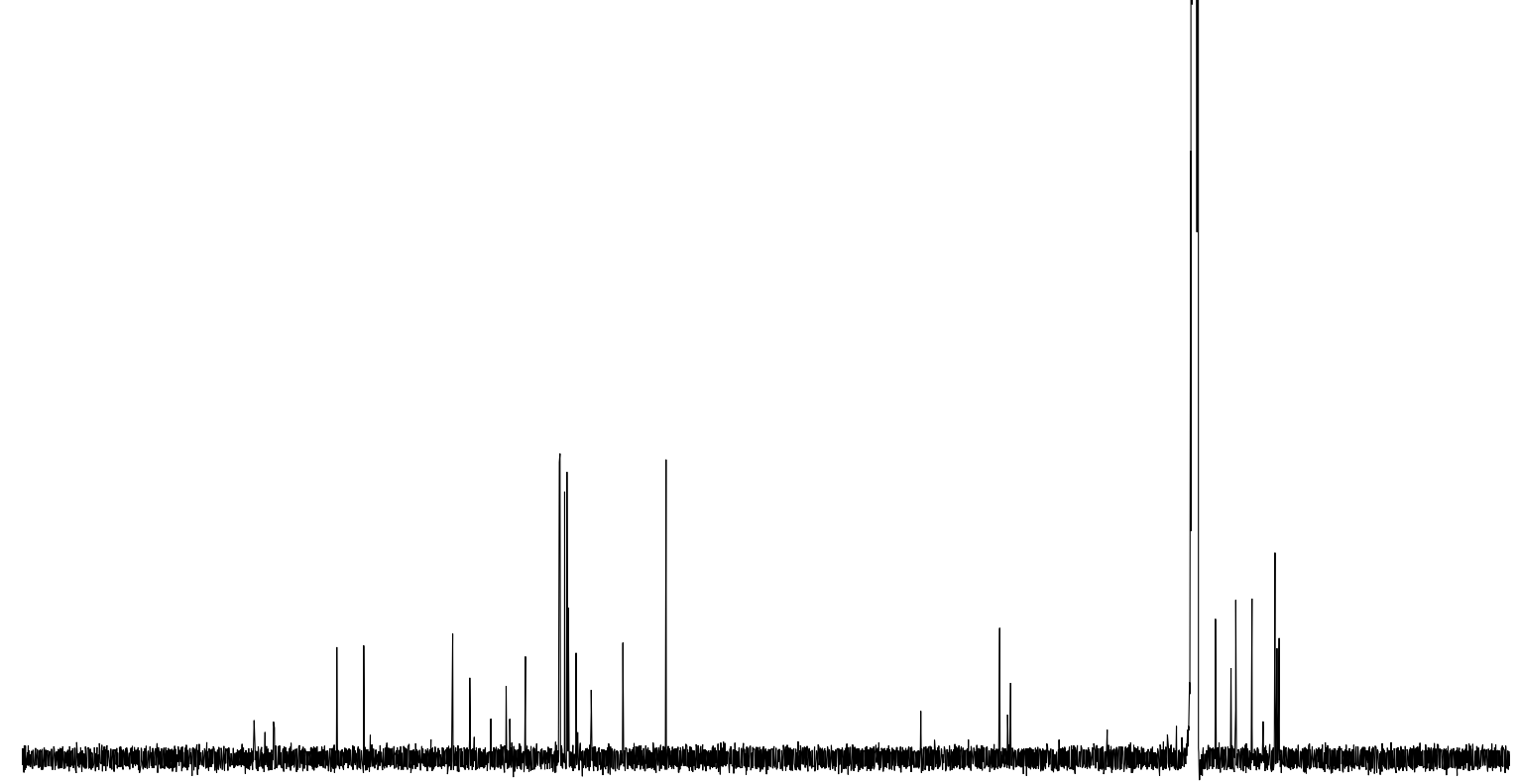

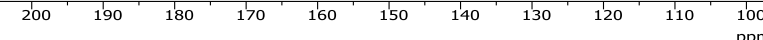

Spectrum 10. ${ }^{13} \mathrm{C}$ NMR $\left(151 \mathrm{MHz}, \mathrm{DMSO}-d_{6}\right)$ of $\mathbf{S 8}$. 


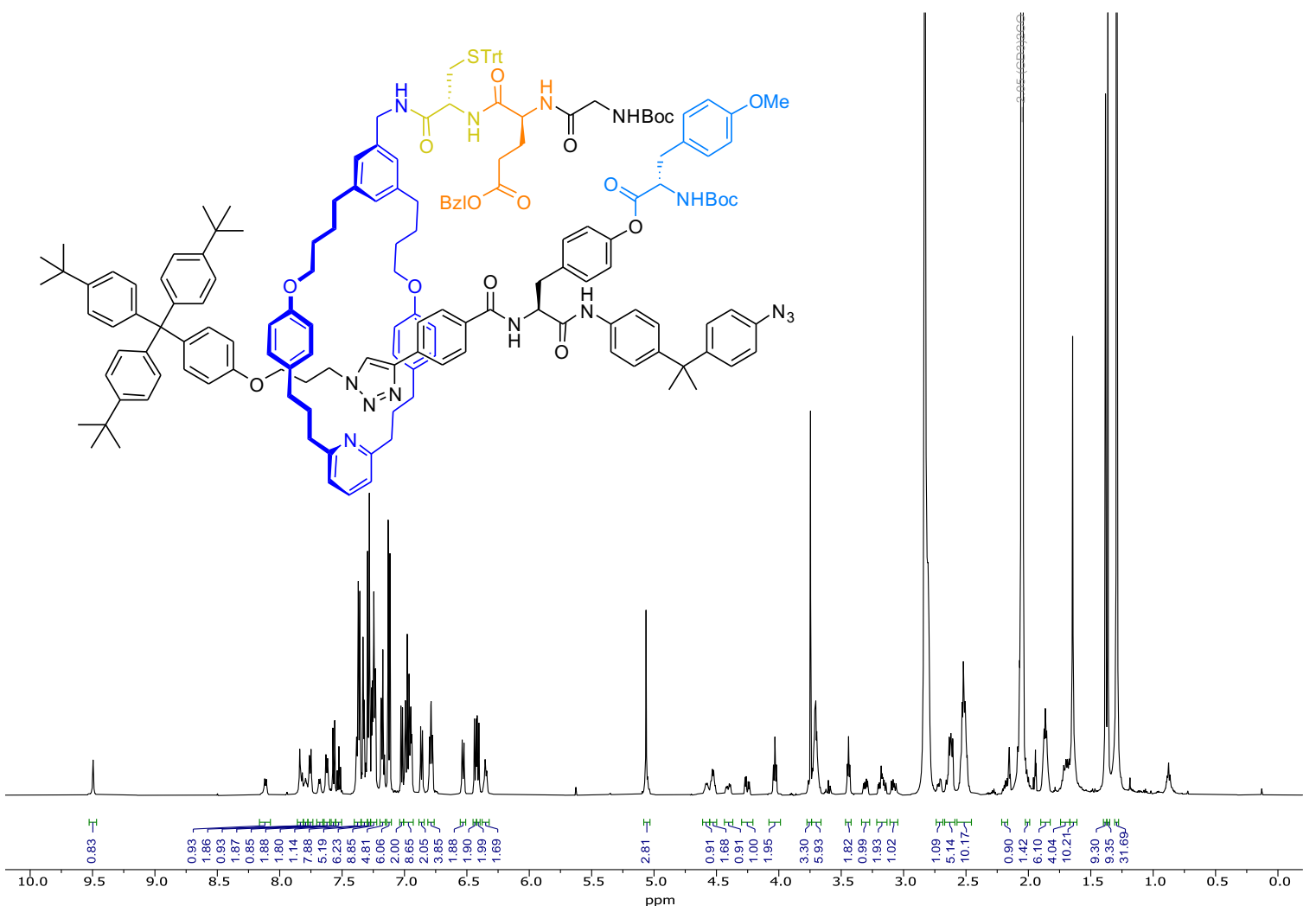

Spectrum 11. ${ }^{1} \mathrm{H}$ NMR $\left(600 \mathrm{MHz}\right.$, Acetone- $\left.d_{6}\right)$ of 3.

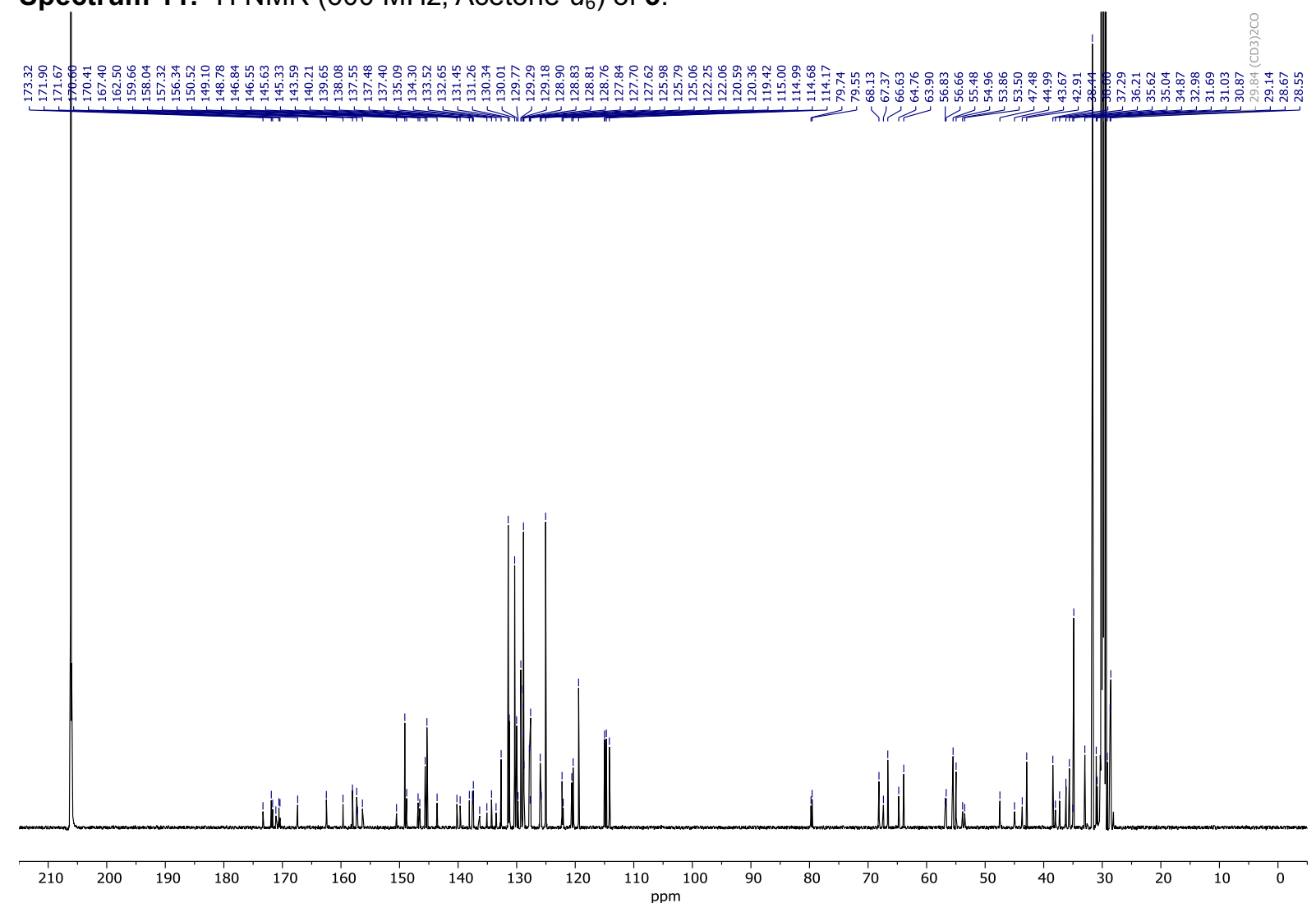

Spectrum 12. ${ }^{13} \mathrm{C}$ NMR $\left(151 \mathrm{MHz}\right.$, Acetone- $\left.d_{6}\right)$ of 3. 

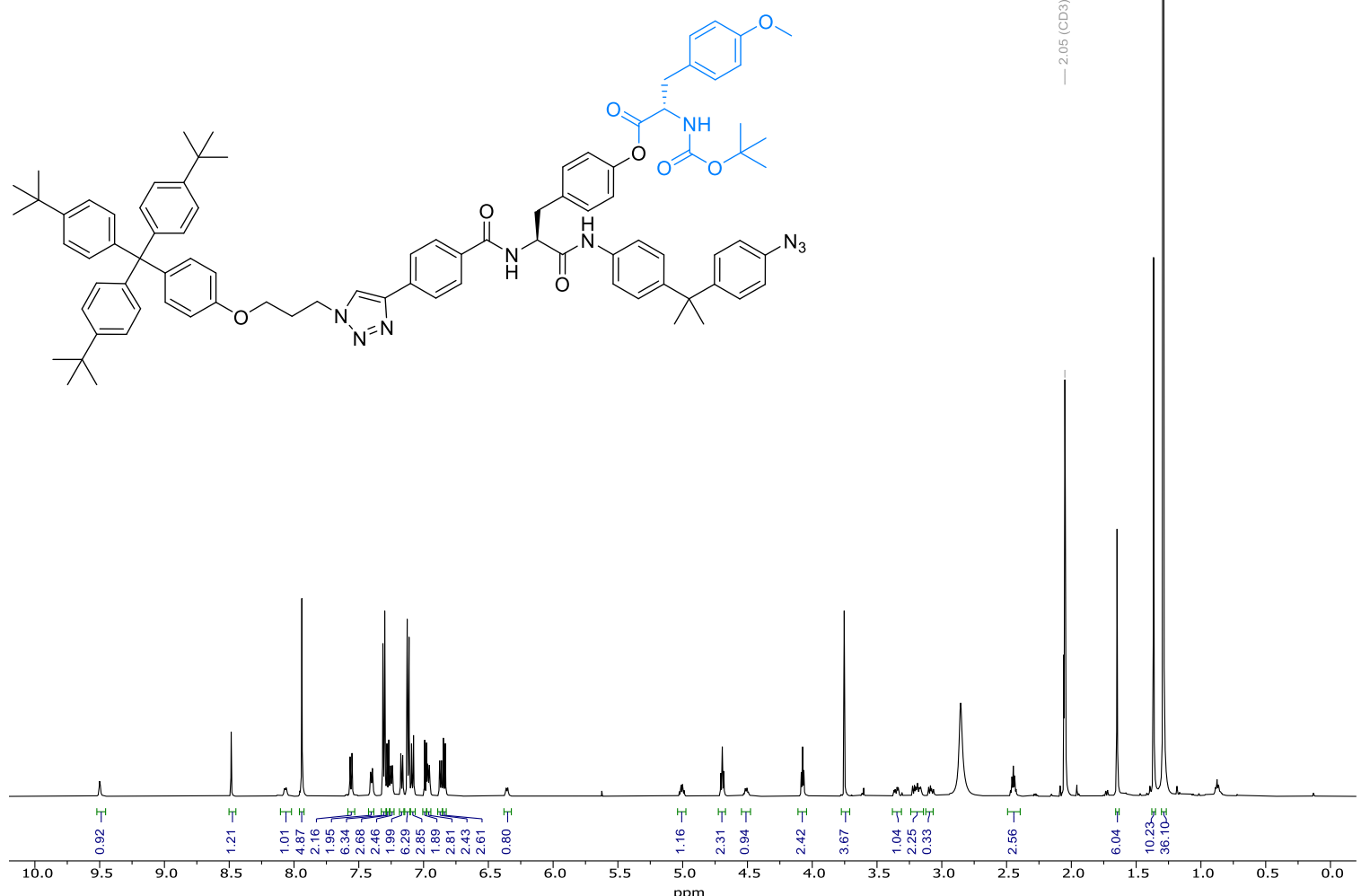

Spectrum 13. ${ }^{1} \mathrm{H}$ NMR $\left(600 \mathrm{MHz}\right.$, Acetone- $\left.d_{6}\right)$ of $\mathbf{S} 10$.

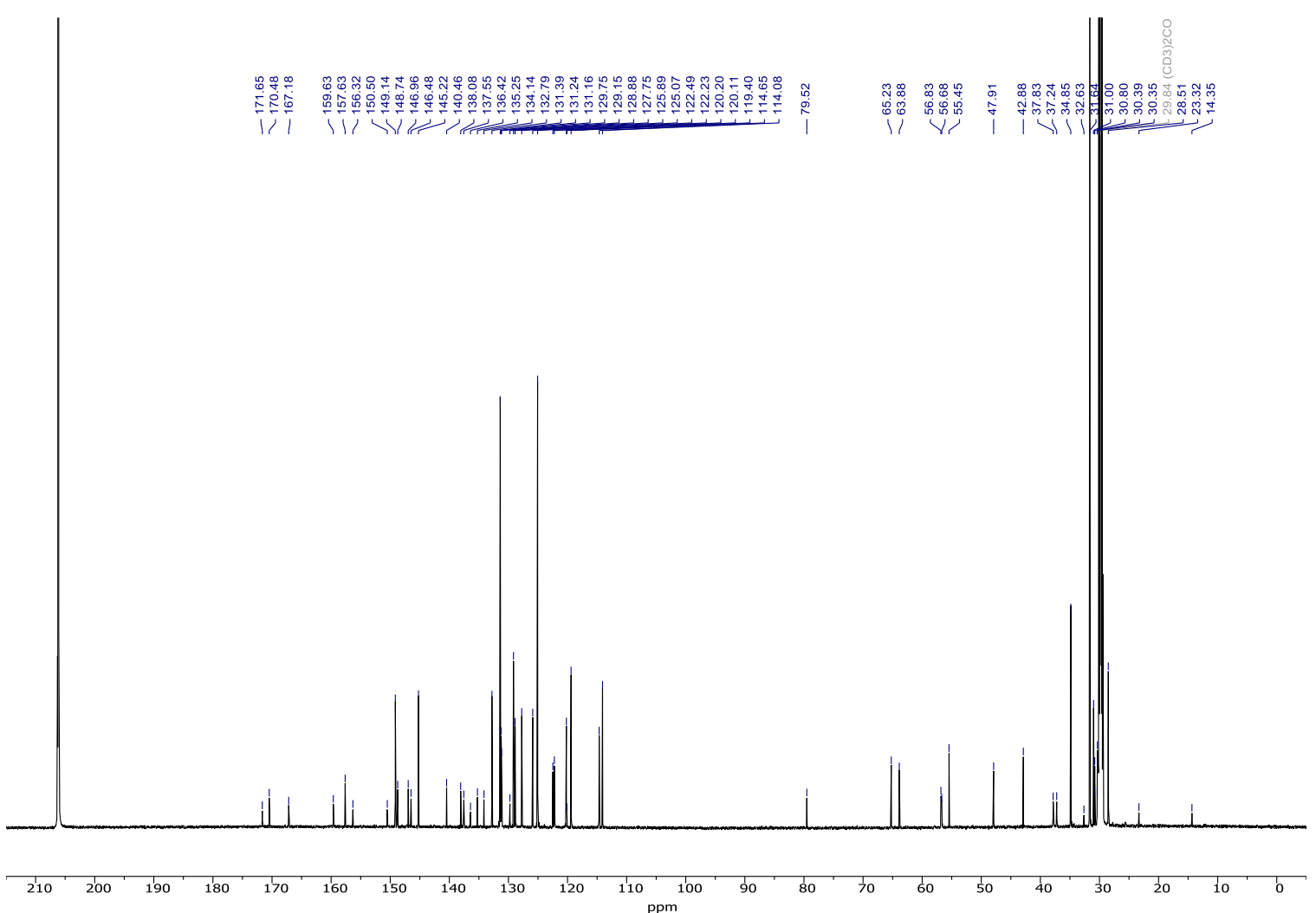

Spectrum 14. ${ }^{13} \mathrm{C}$ NMR $\left(151 \mathrm{MHz}\right.$, Acetone- $\left.d_{6}\right)$ of $\mathbf{S 1 0}$. 


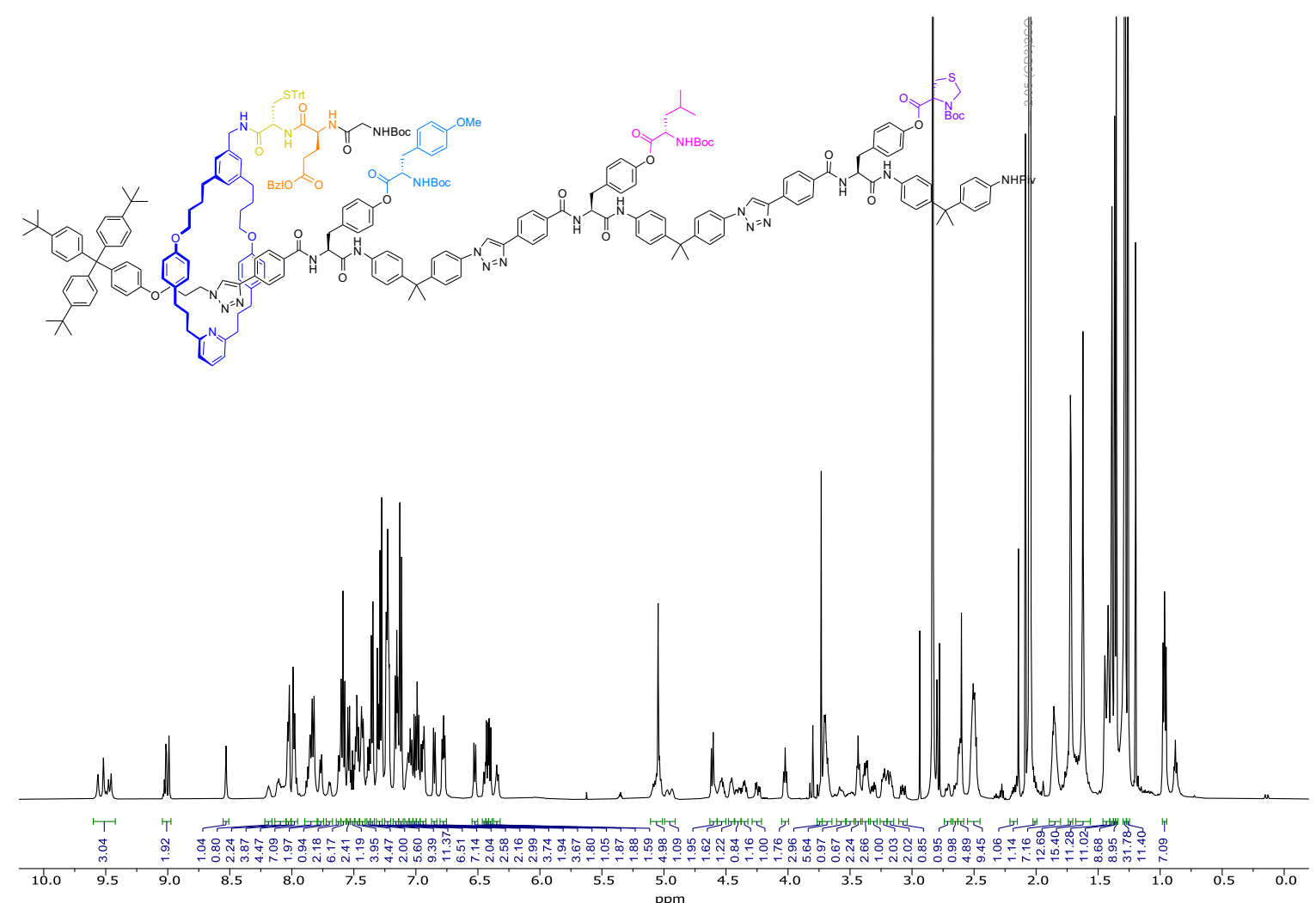

Spectrum 15. ${ }^{1} \mathrm{H}$ NMR $\left(600 \mathrm{MHz}\right.$, Acetone- $\left.d_{6}\right)$ of 1.

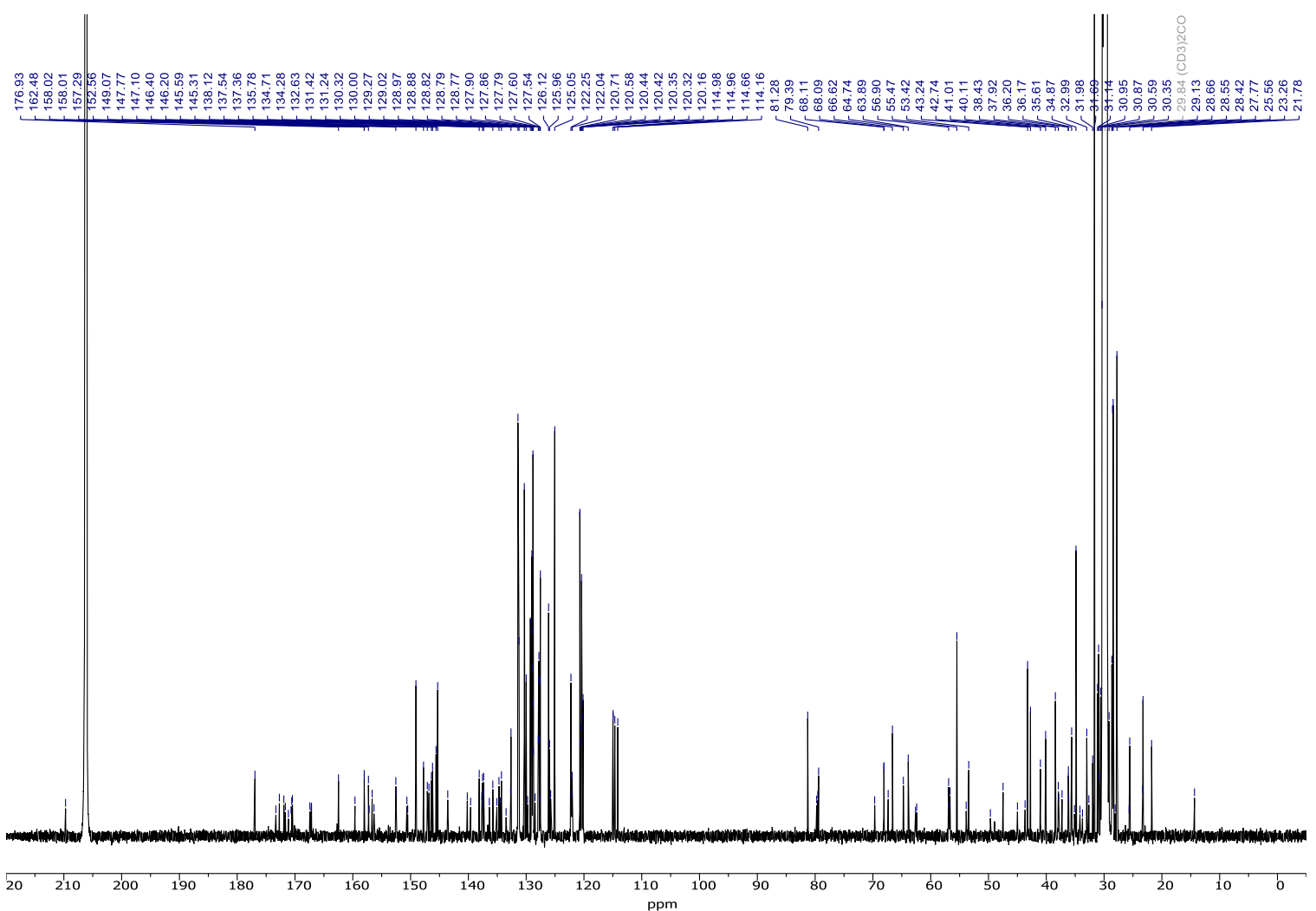

Spectrum 16. ${ }^{13} \mathrm{C}$ NMR $\left(151 \mathrm{MHz}\right.$, Acetone- $\left.d_{6}\right)$ of 1 . 
SI Echavarren et al, 'Sequence-Selective Decapeptide Synthesis by the Parallel Operation of ...'

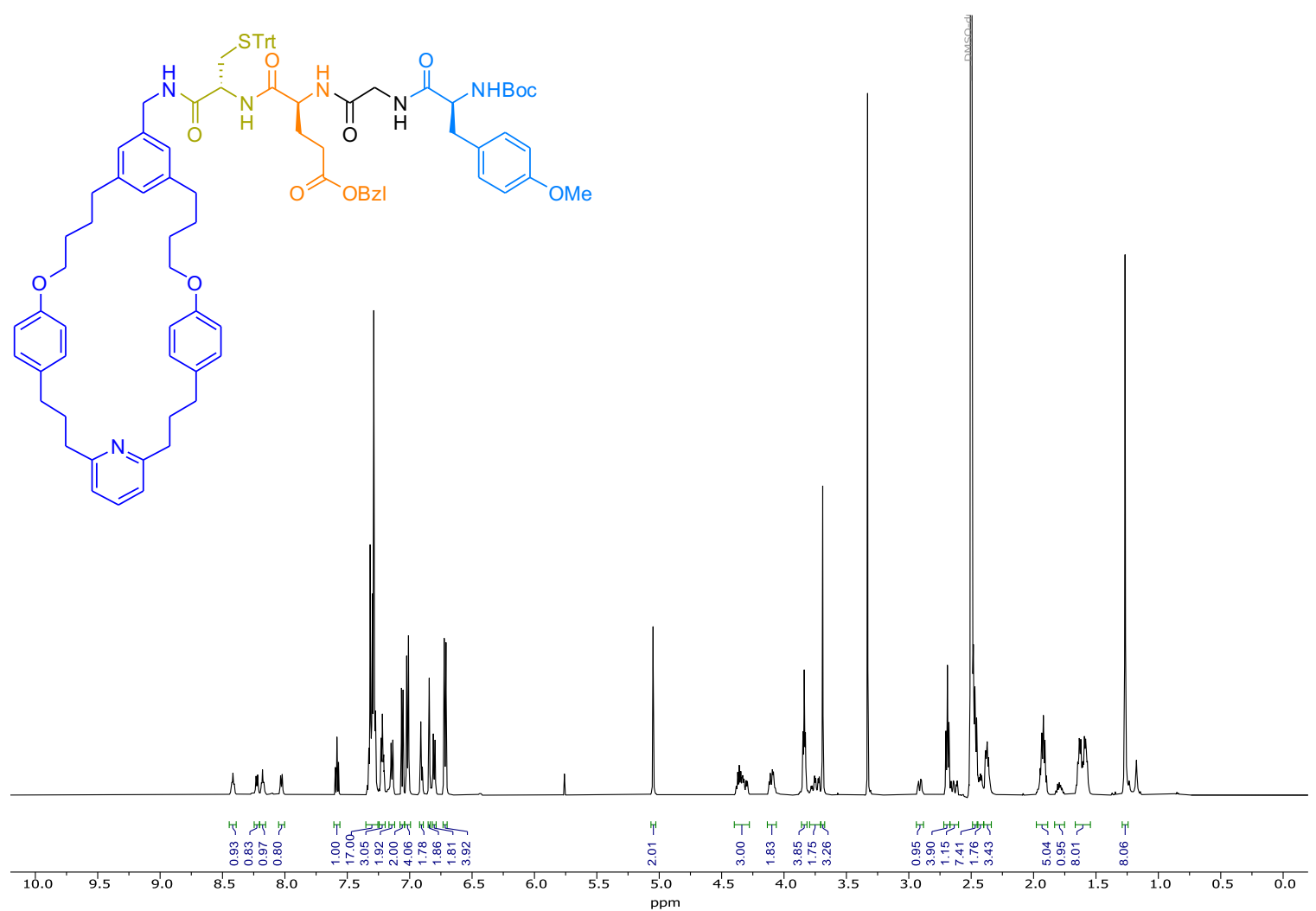

Spectrum 17. ${ }^{1} \mathrm{H}$ NMR $\left(600 \mathrm{MHz}, \mathrm{DMSO}-d_{6}\right)$ of $\mathbf{S} 13$.

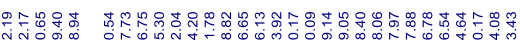

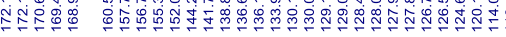

作

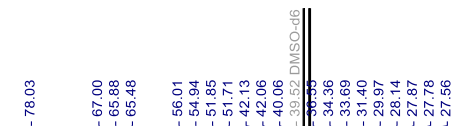

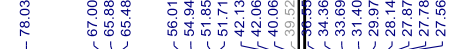

$र \backslash 1111$

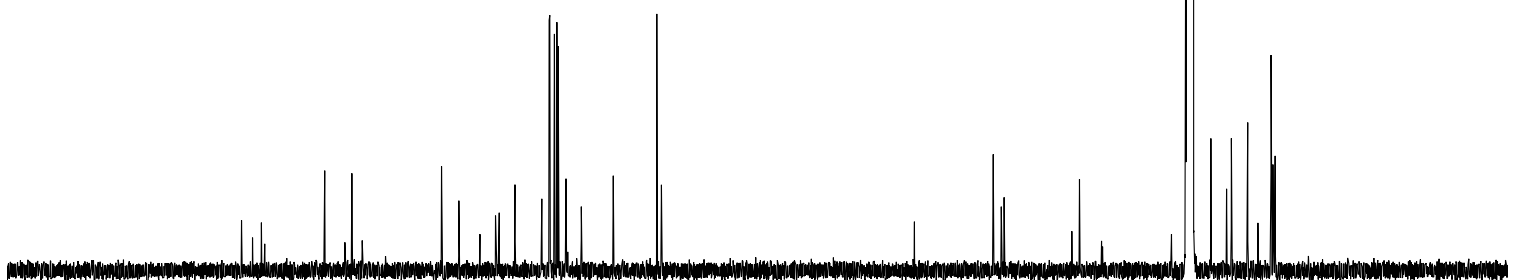

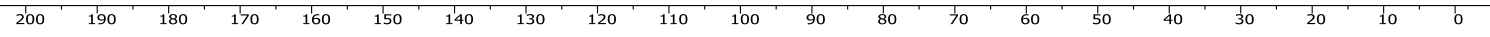

Spectrum 18. ${ }^{13} \mathrm{C}$ NMR $\left(151 \mathrm{MHz}\right.$, DMSO- $\left.d_{6}\right)$ of $\mathbf{S} 13$. 


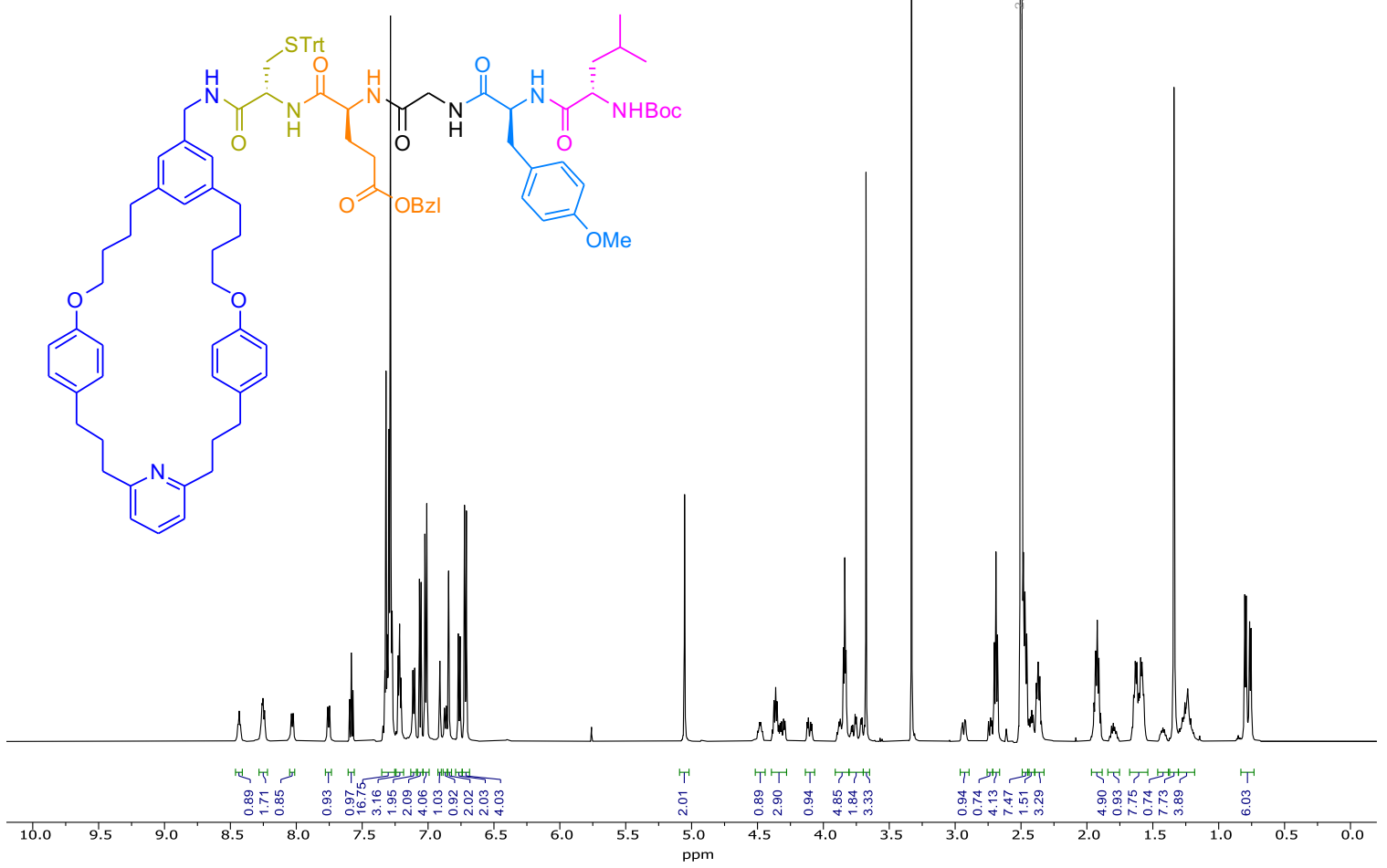

Spectrum 19. ${ }^{1} \mathrm{H}$ NMR $\left(600 \mathrm{MHz}, \mathrm{DMSO}-d_{6}\right)$ of $\mathbf{S} 14$.

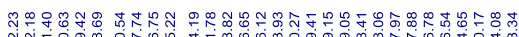

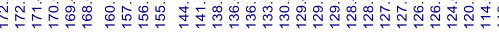

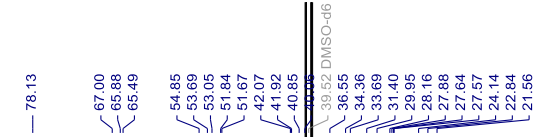

$41 / 11$

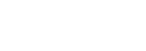

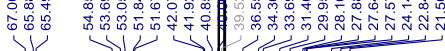

(1)

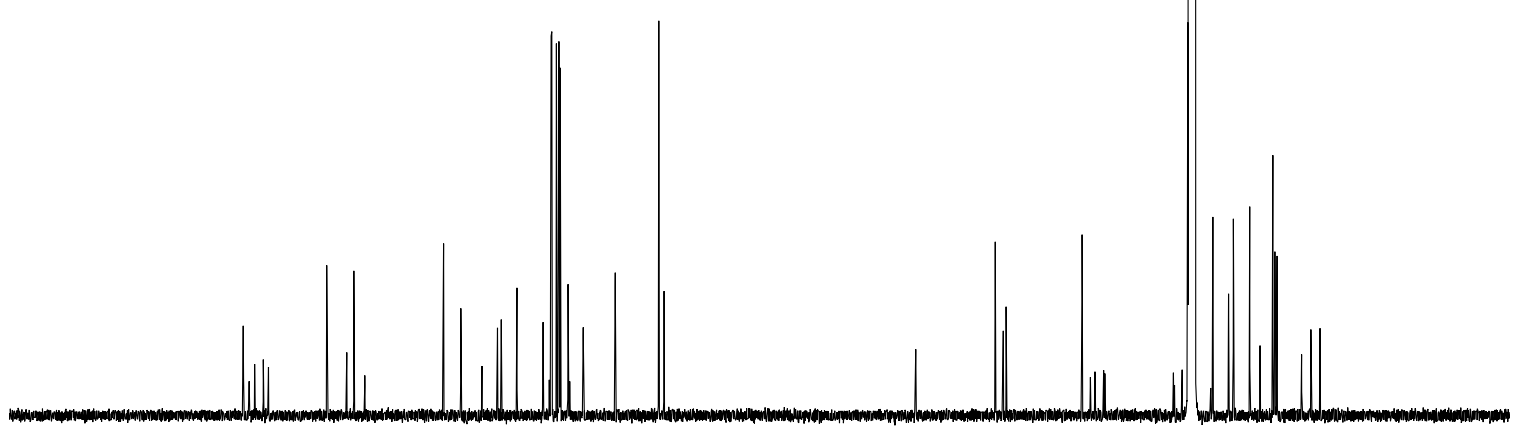

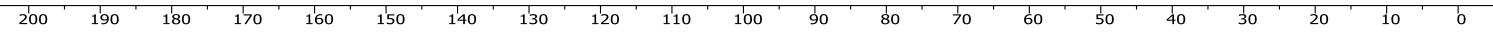

Spectrum 20. ${ }^{13} \mathrm{C}$ NMR $\left(151 \mathrm{MHz}\right.$, DMSO- $\left.d_{6}\right)$ of $\mathbf{S} 14$. 


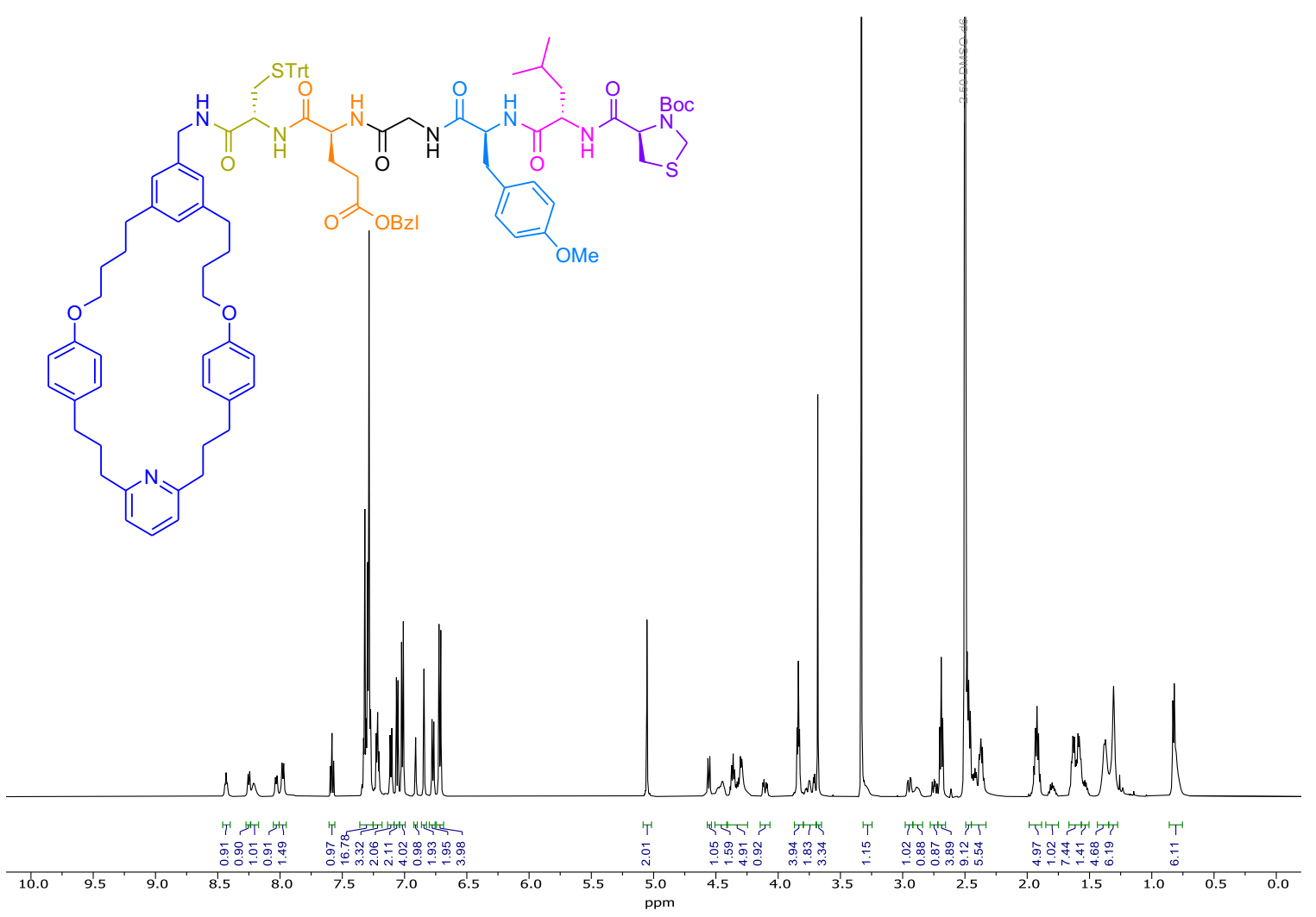

Spectrum 21. ${ }^{1} \mathrm{H}$ NMR $\left(600 \mathrm{MHz}, \mathrm{DMSO}-d_{6}\right)$ of $\mathbf{S} 15$.

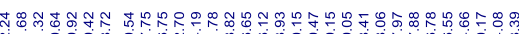
Nir.

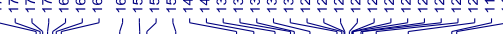
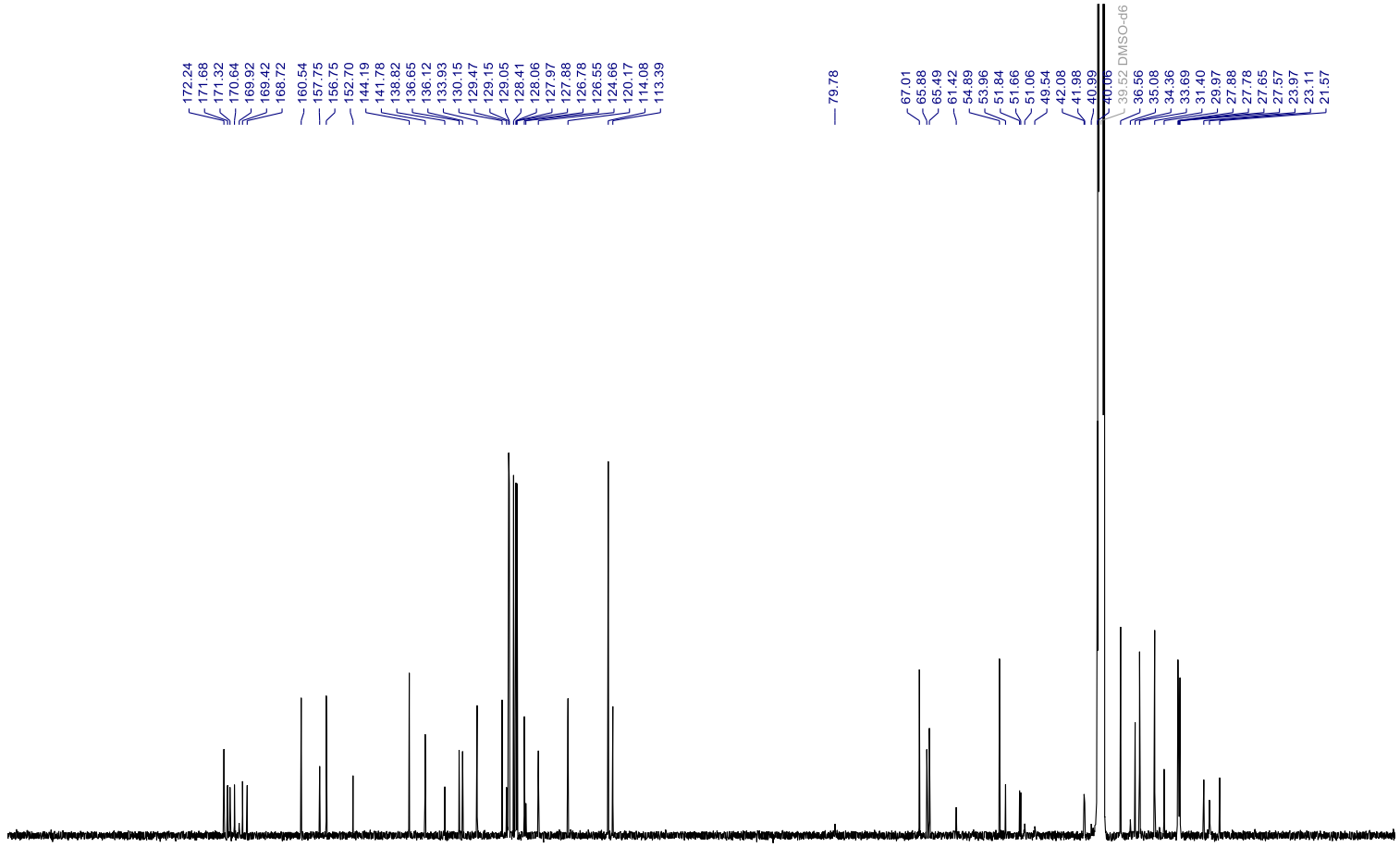

$\begin{array}{lllllllllll}2^{\prime} 00 & 190 & 180 & 170 & 160 & 1^{\prime} 50 & 1^{\prime} 40 & 1^{\prime} 30 & 120 & 110 & 1\end{array}$

Spectrum 22. ${ }^{13} \mathrm{C}$ NMR $\left(151 \mathrm{MHz}, \mathrm{DMSO}-d_{6}\right)$ of $\mathbf{S 1 5}$. 
SI Echavarren et al, 'Sequence-Selective Decapeptide Synthesis by the Parallel Operation of ...'

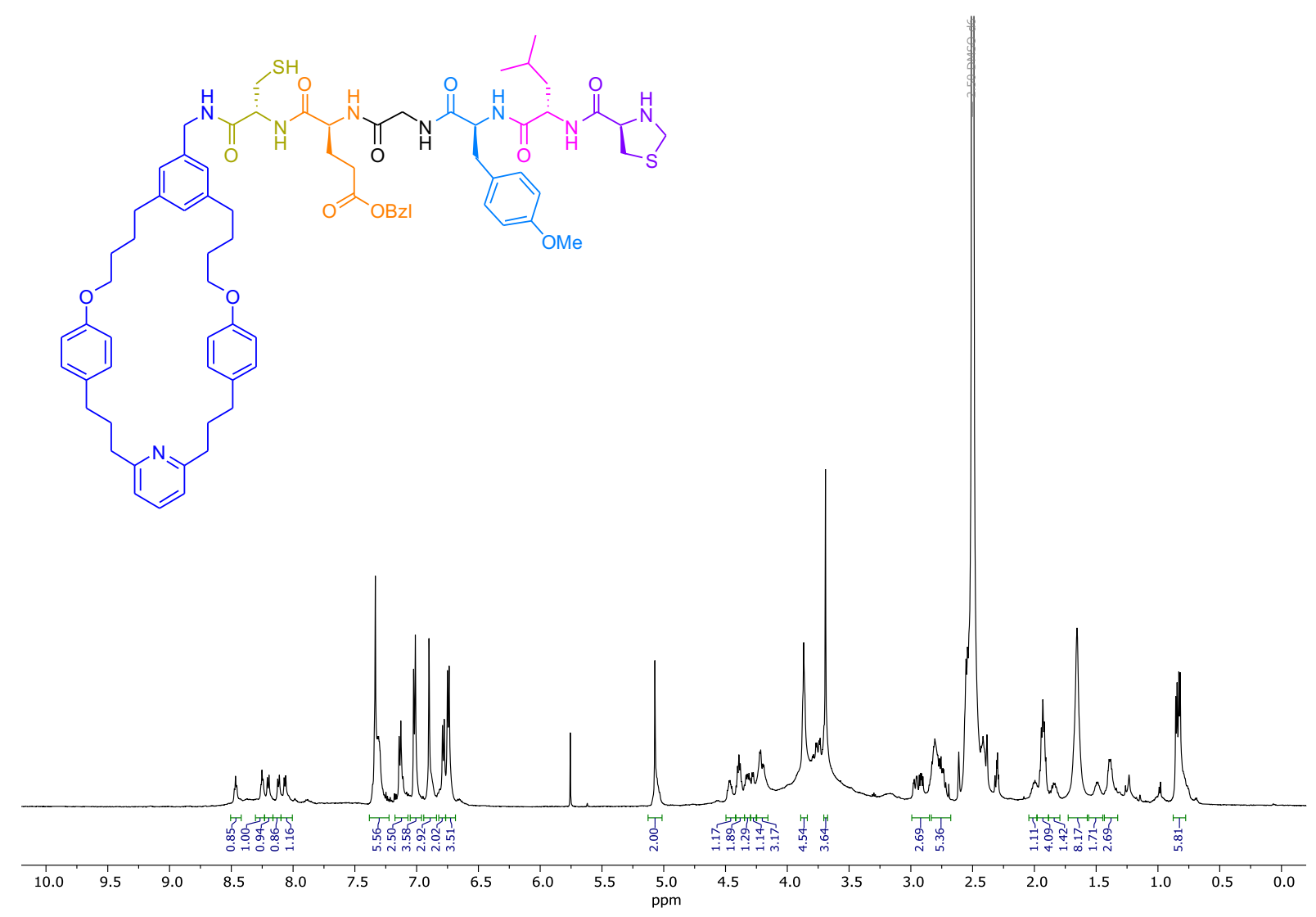

Spectrum 23. ${ }^{1} \mathrm{H}$ NMR $\left(600 \mathrm{MHz}\right.$, DMSO- $\left.d_{6}\right)$ of 7. 


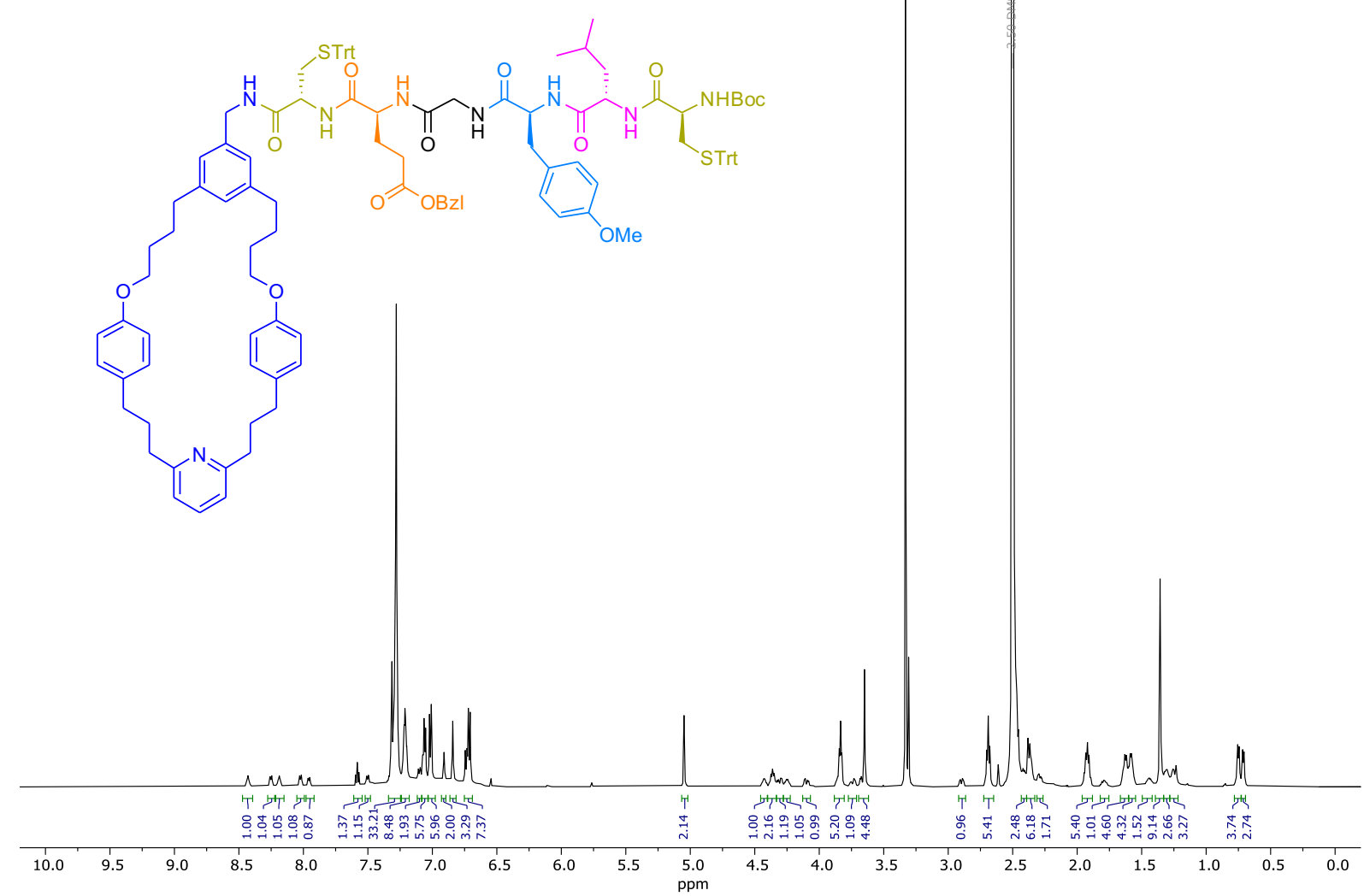

Spectrum 24. ${ }^{1} \mathrm{H}$ NMR $\left(600 \mathrm{MHz}, \mathrm{DMSO}-d_{6}\right)$ of $\mathbf{S} 16$.
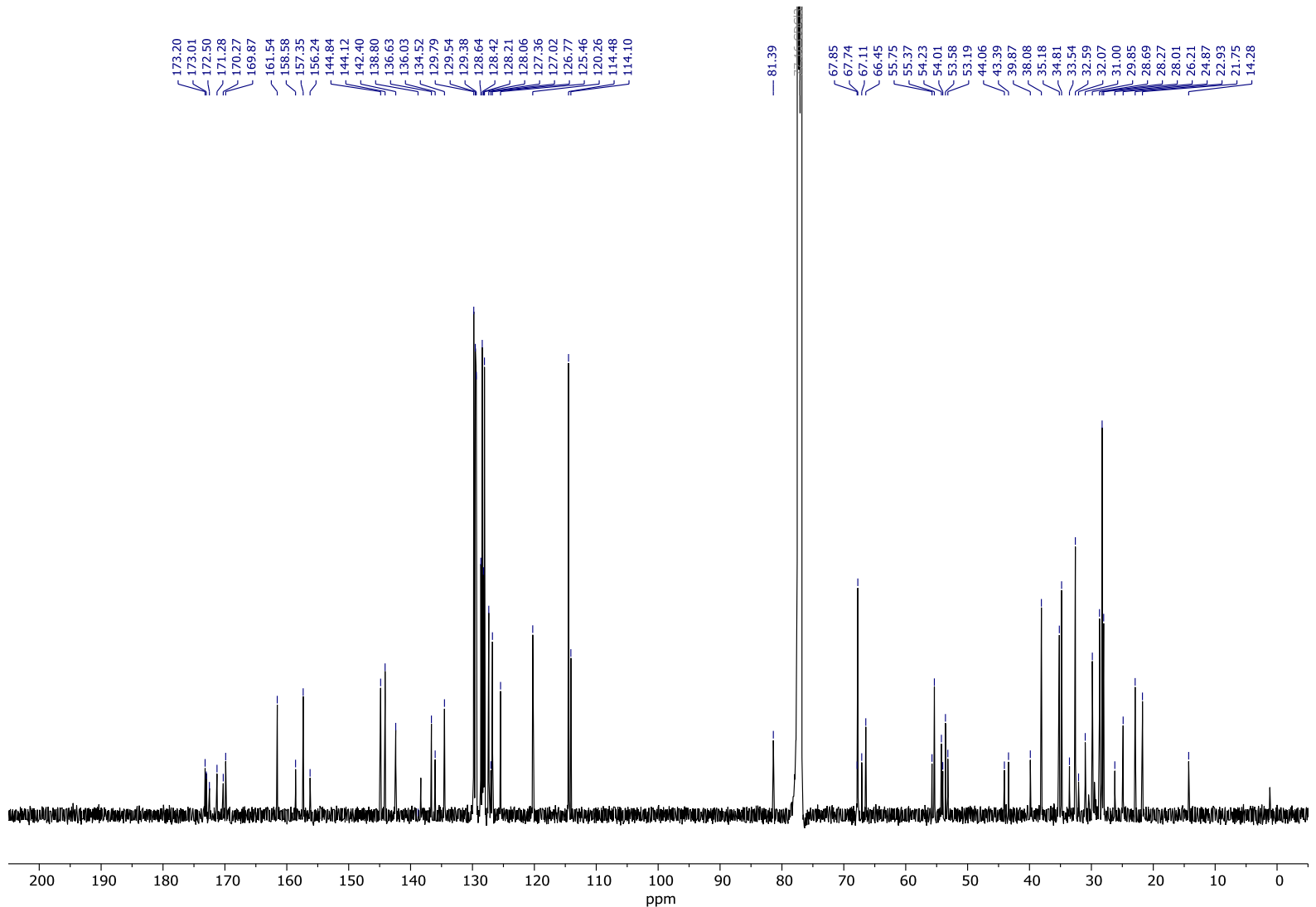

Spectrum 25. ${ }^{13} \mathrm{C}$ NMR $\left(151 \mathrm{MHz}, \mathrm{CDCl}_{3}\right)$ of $\mathbf{S} 16$. 
SI Echavarren et al, 'Sequence-Selective Decapeptide Synthesis by the Parallel Operation of ...'

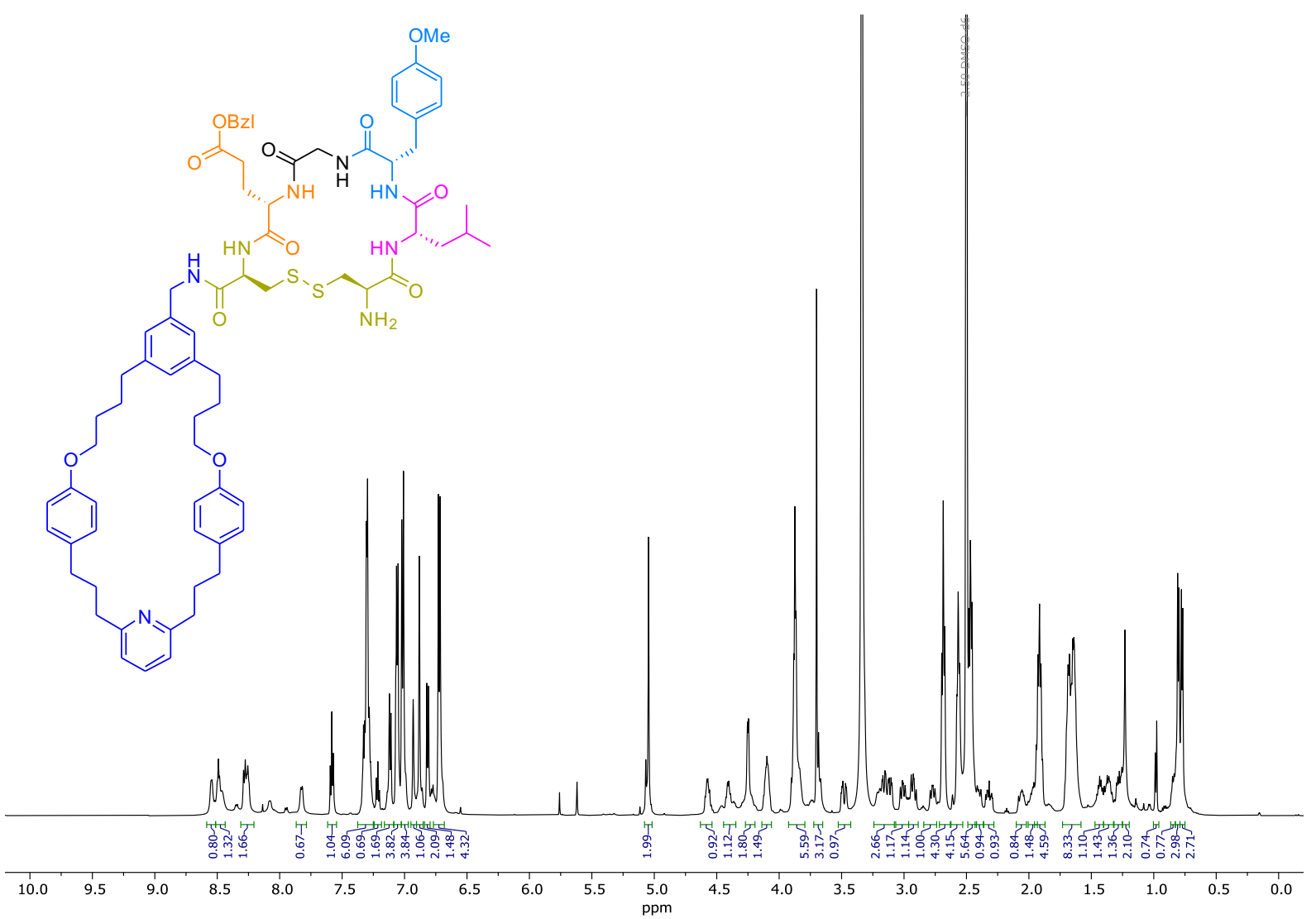

Spectrum 26. ${ }^{1} \mathrm{H}$ NMR $\left(600 \mathrm{MHz}, \mathrm{DMSO}-d_{6}\right)$ of $\mathbf{S} 17$.

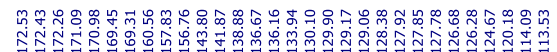

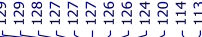

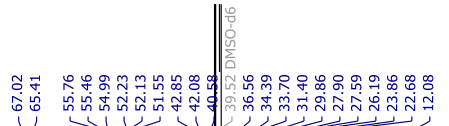

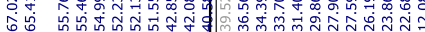

4 पा

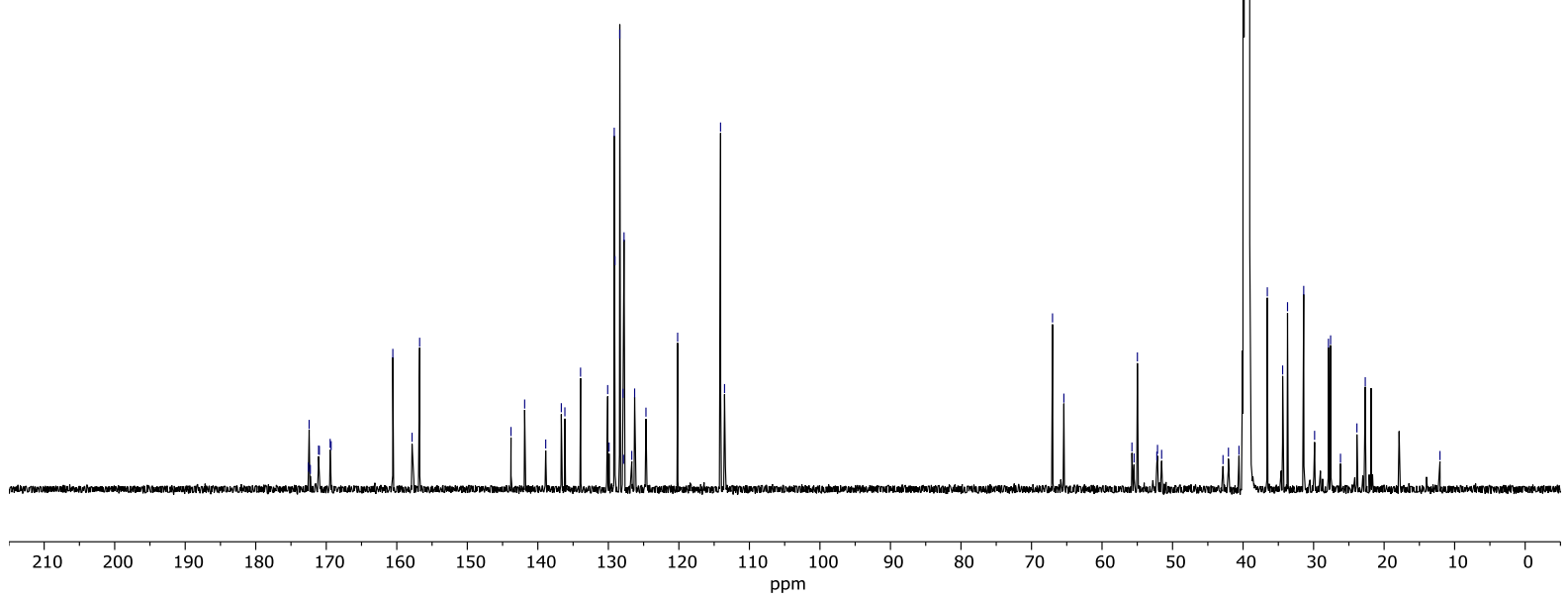

Spectrum $27 .{ }^{13} \mathrm{C}$ NMR $\left(151 \mathrm{MHz}\right.$, DMSO- $\left.d_{6}\right)$ of $\mathbf{S} 17$. 


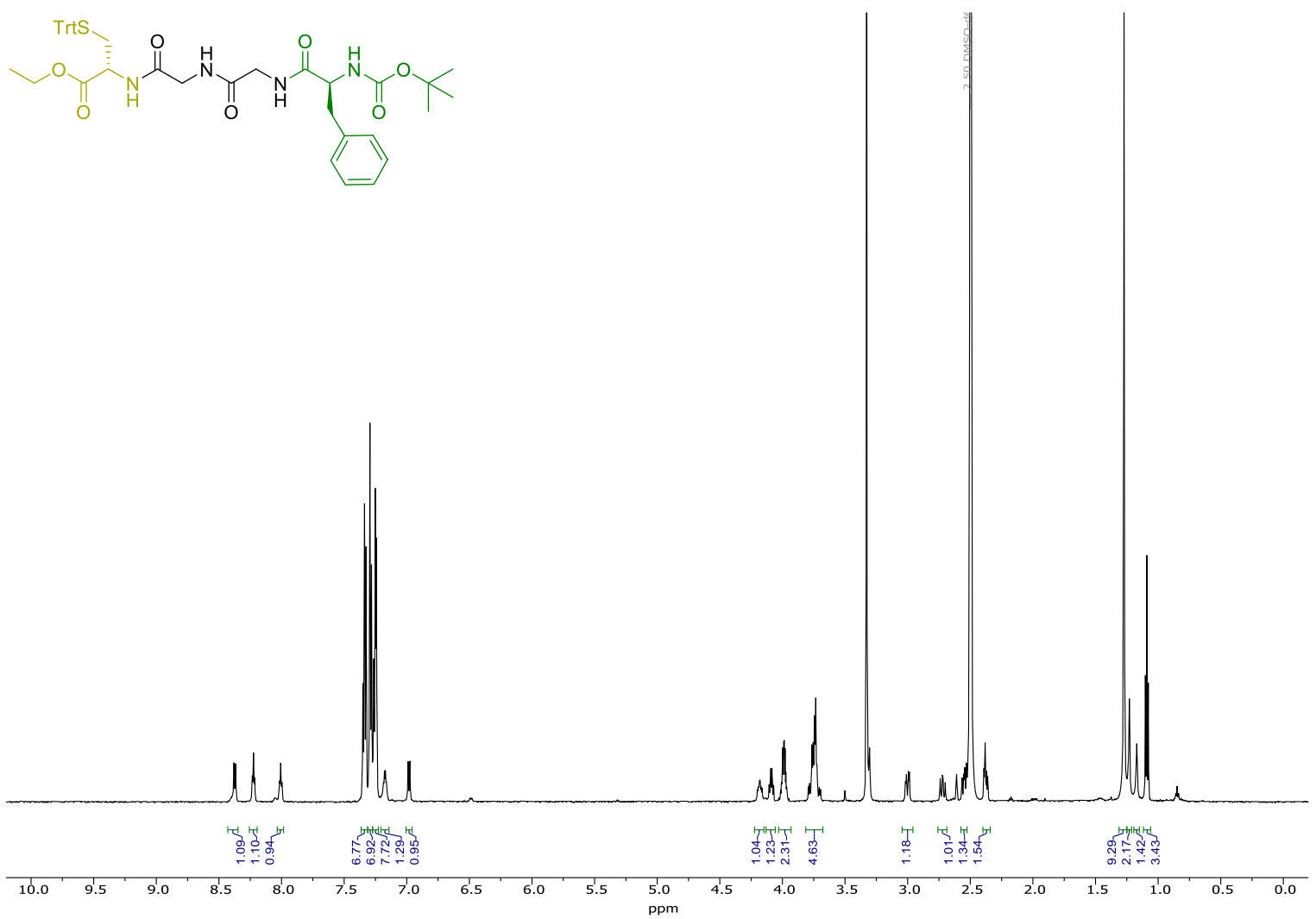

Spectrum 28. ${ }^{1} \mathrm{H}$ NMR $\left(600 \mathrm{MHz}, \mathrm{DMSO}-d_{6}\right)$ of $\mathbf{S} 19$.

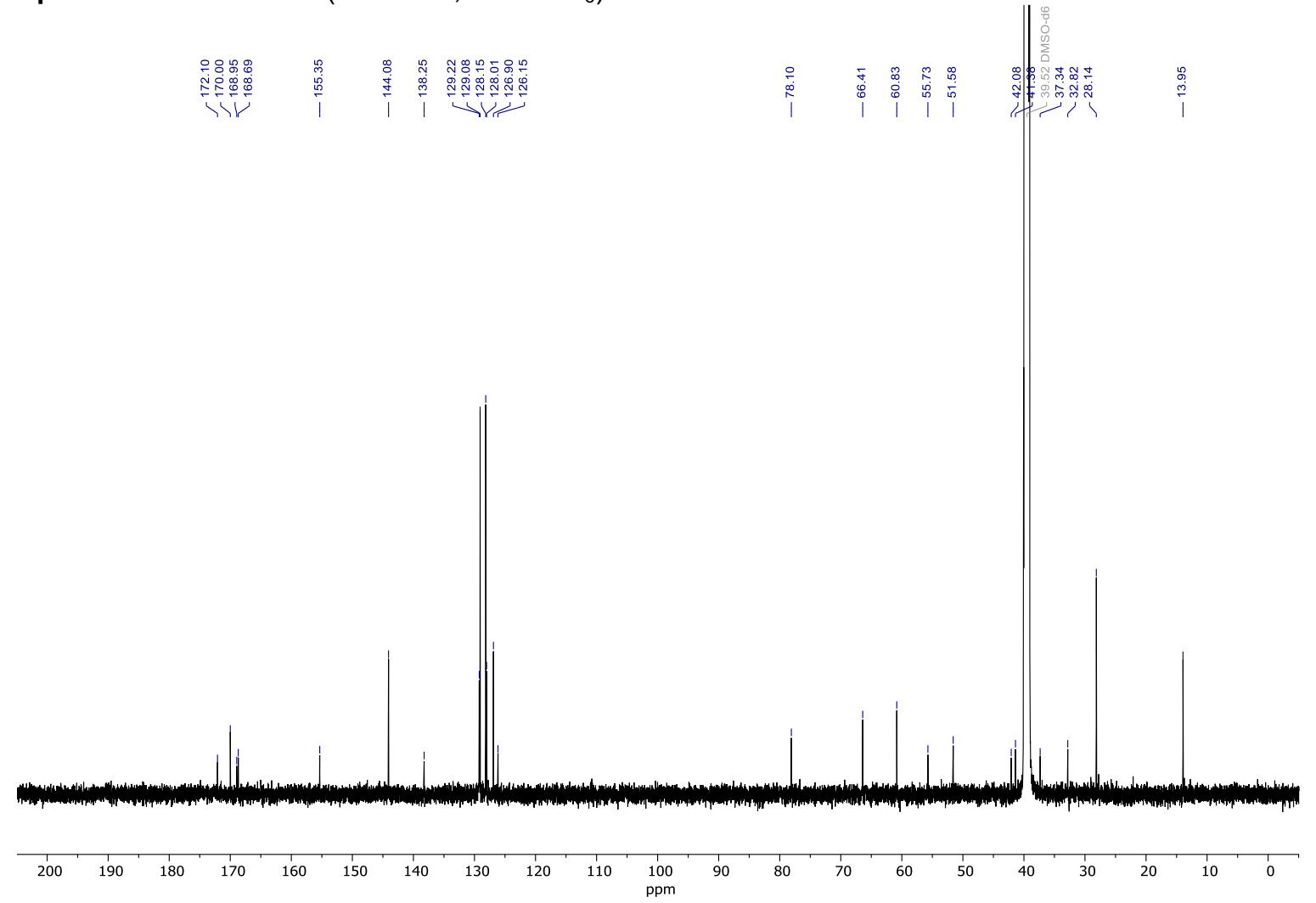

Spectrum 29. ${ }^{13} \mathrm{C}$ NMR $\left(151 \mathrm{MHz}\right.$, DMSO- $\left.d_{6}\right)$ of $\mathbf{S 1 9}$. 
SI Echavarren et al, 'Sequence-Selective Decapeptide Synthesis by the Parallel Operation of ...'

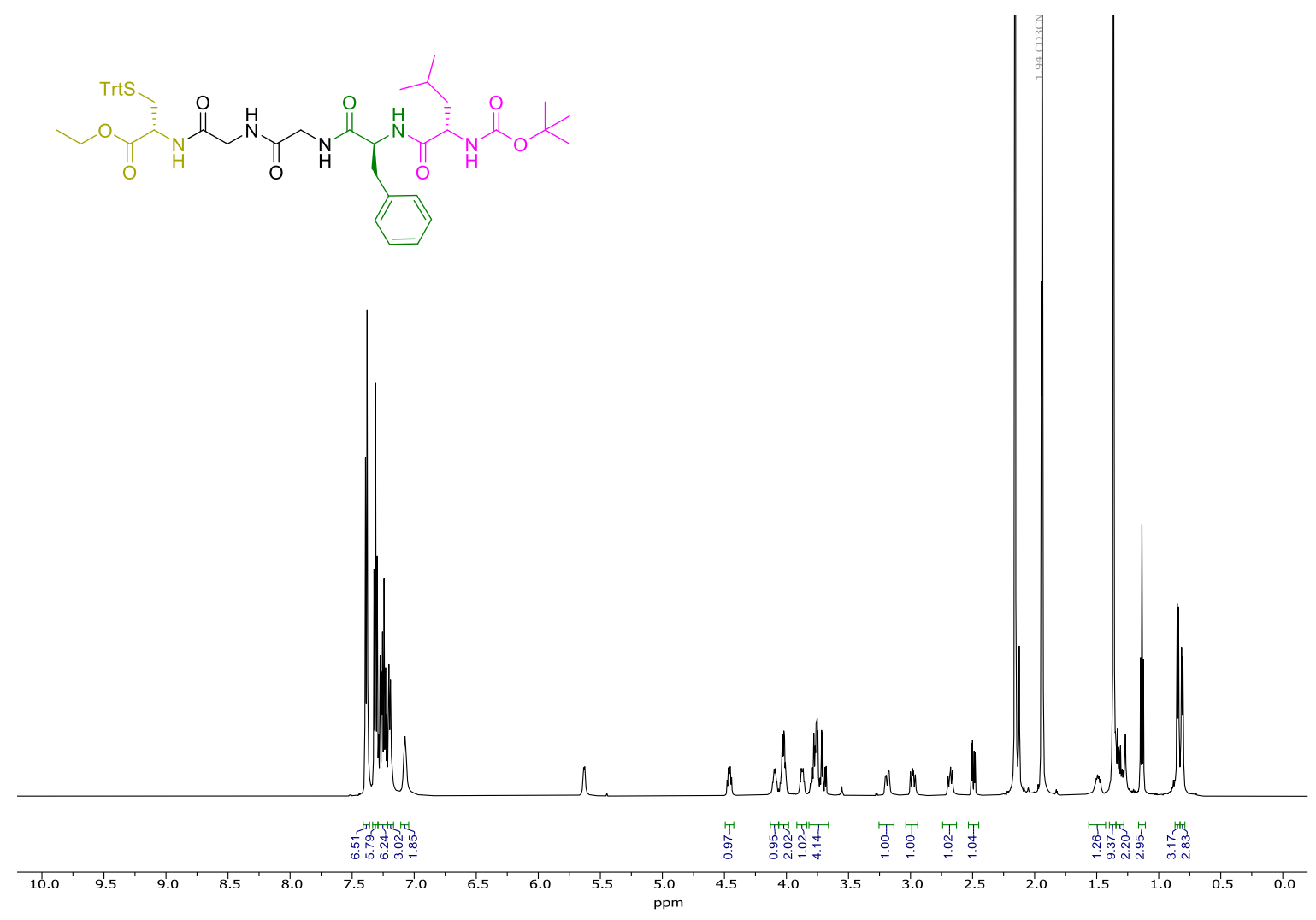

Spectrum 30. ${ }^{1} \mathrm{H}$ NMR $\left(600 \mathrm{MHz}\right.$, Acetonitrile- $\left.d_{3}\right)$ of $\mathbf{S 2 0}$.

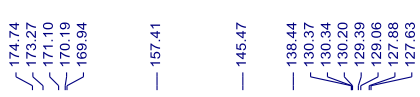

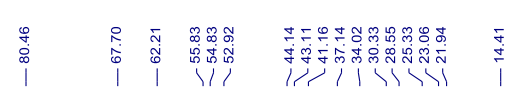

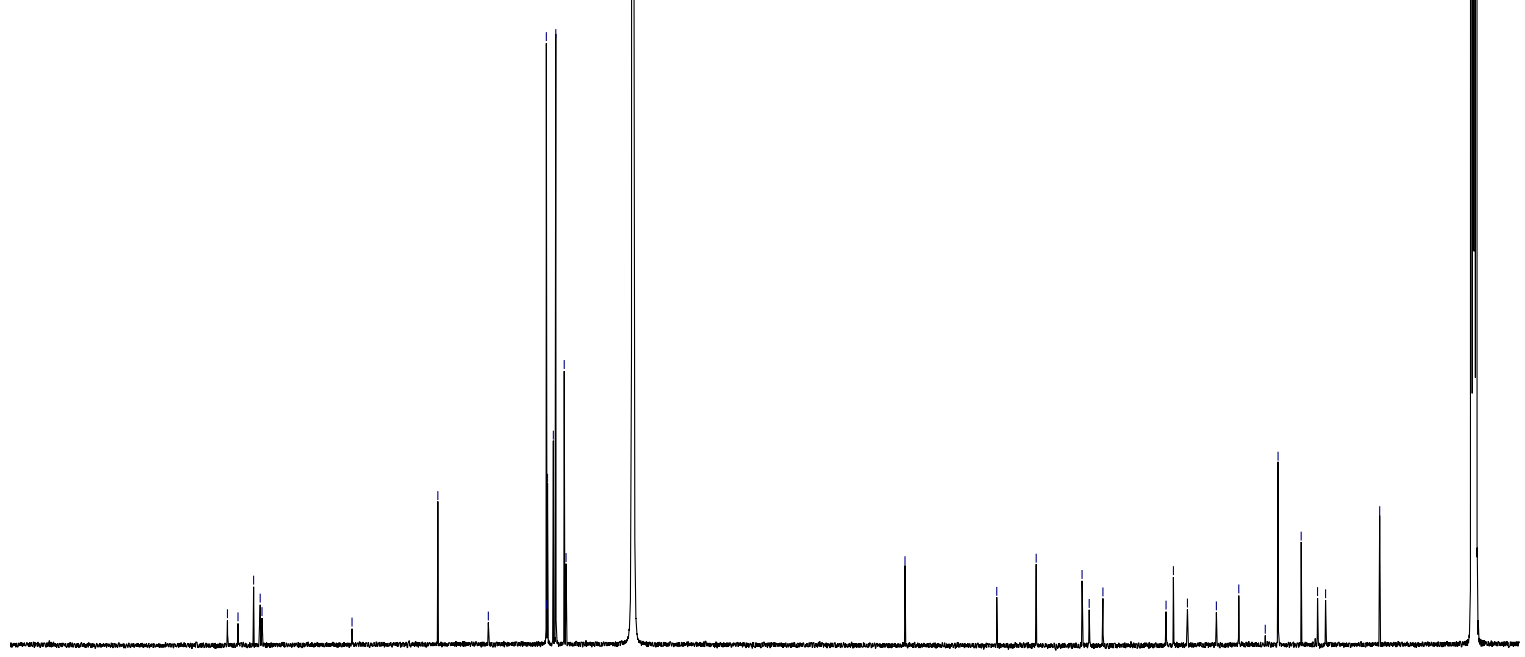

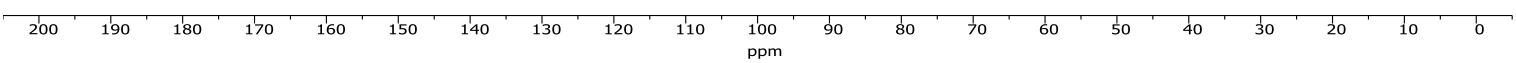

Spectrum 31. ${ }^{13} \mathrm{C}$ NMR $\left(151 \mathrm{MHz}\right.$, Acetonitrile- $\left.d_{3}\right)$ of $\mathbf{S 2 0}$. 
SI Echavarren et al, 'Sequence-Selective Decapeptide Synthesis by the Parallel Operation of ...'

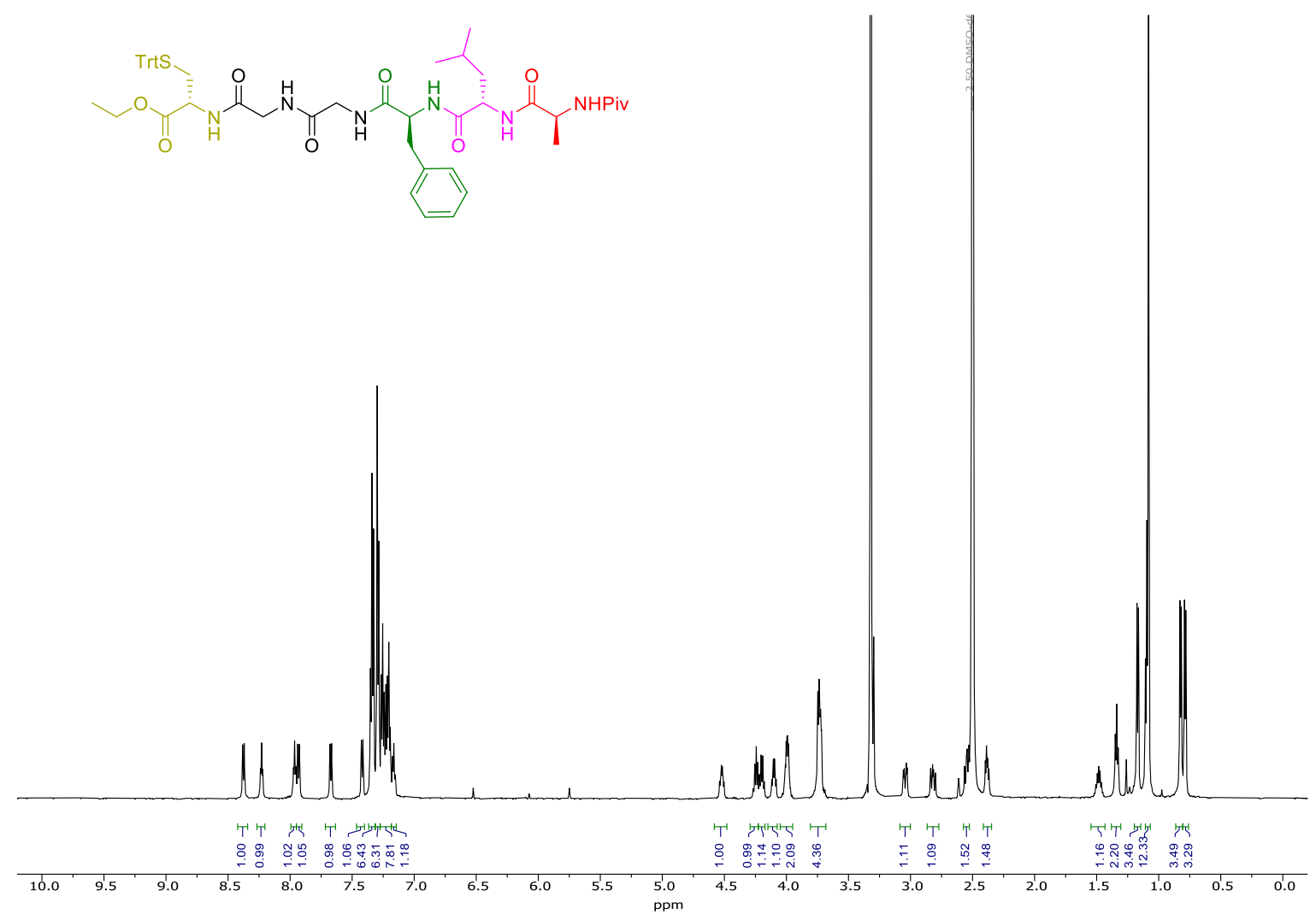

Spectrum 32. ${ }^{1} \mathrm{H}$ NMR $\left(600 \mathrm{MHz}, \mathrm{DMSO}-d_{6}\right)$ of $\mathbf{S 2 1}$.

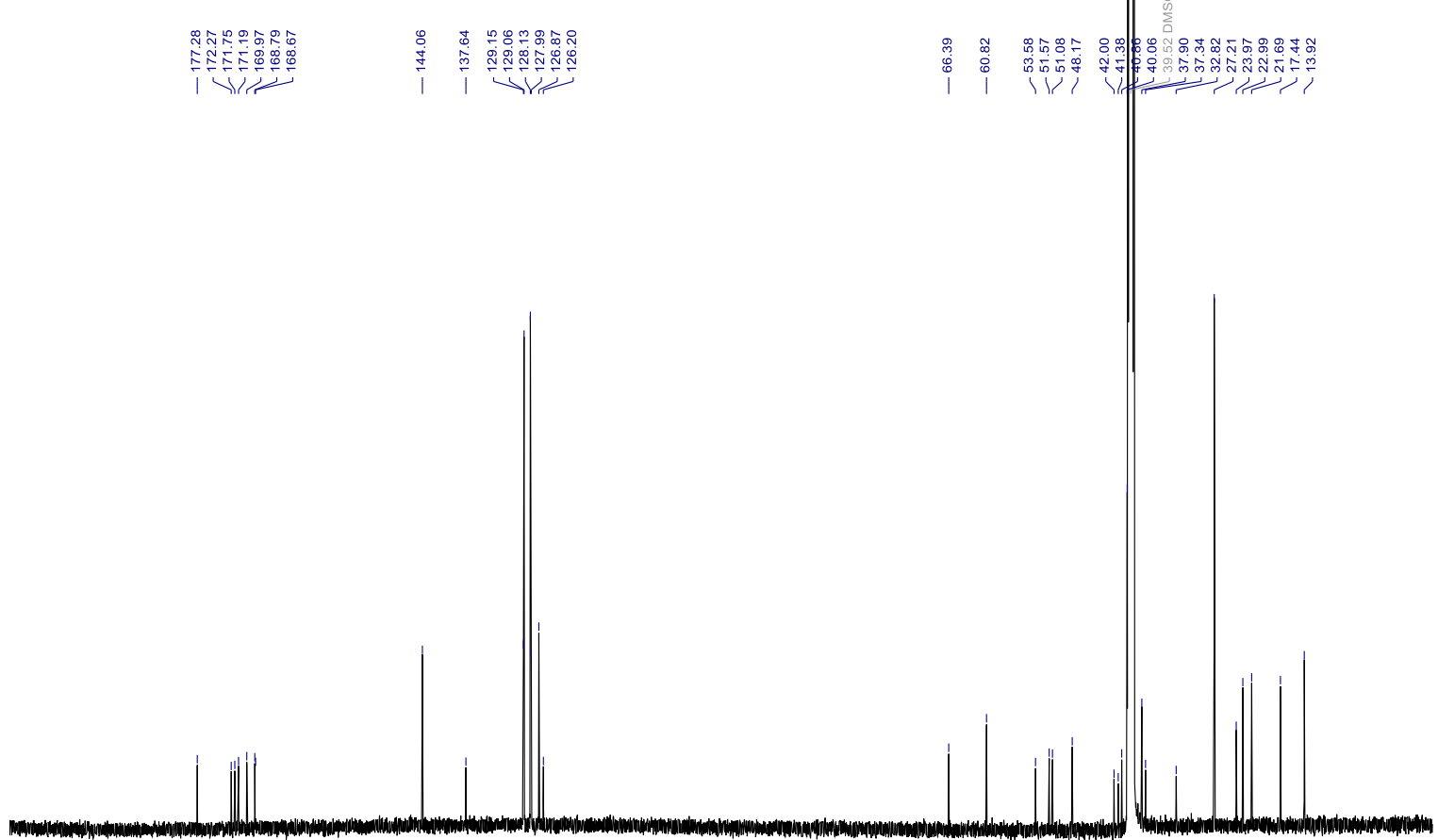

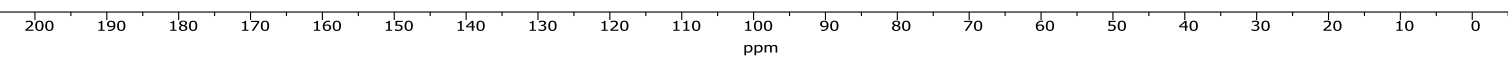

Spectrum 33. ${ }^{13} \mathrm{C}$ NMR $\left(151 \mathrm{MHz}\right.$, DMSO- $\left.d_{6}\right)$ of $\mathbf{S 2 1}$. 
SI Echavarren et al, 'Sequence-Selective Decapeptide Synthesis by the Parallel Operation of ...'

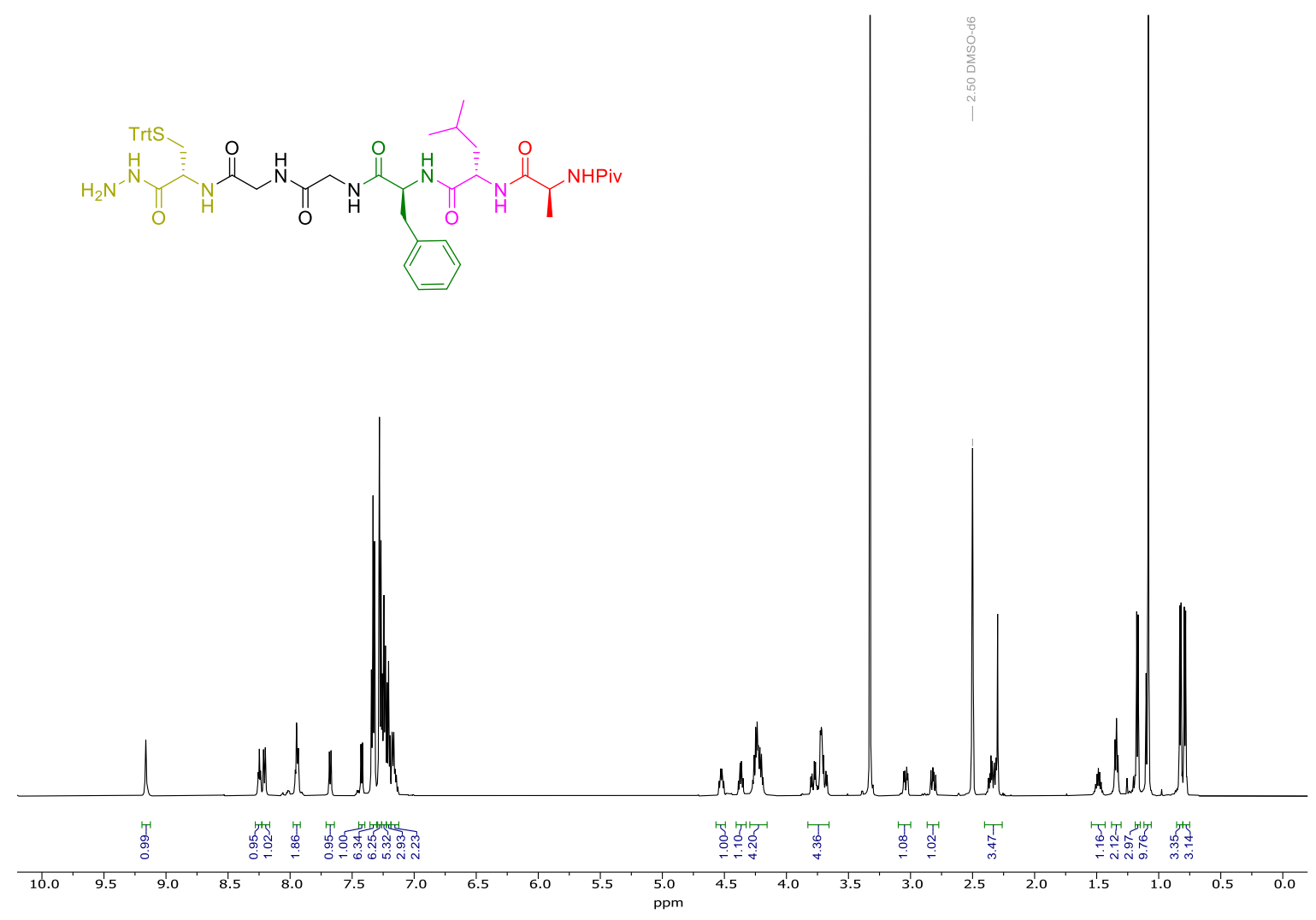

Spectrum 34. ${ }^{1} \mathrm{H}$ NMR $\left(600 \mathrm{MHz}\right.$, DMSO- $\left.d_{6}\right)$ of $\mathbf{S 2 2}$.

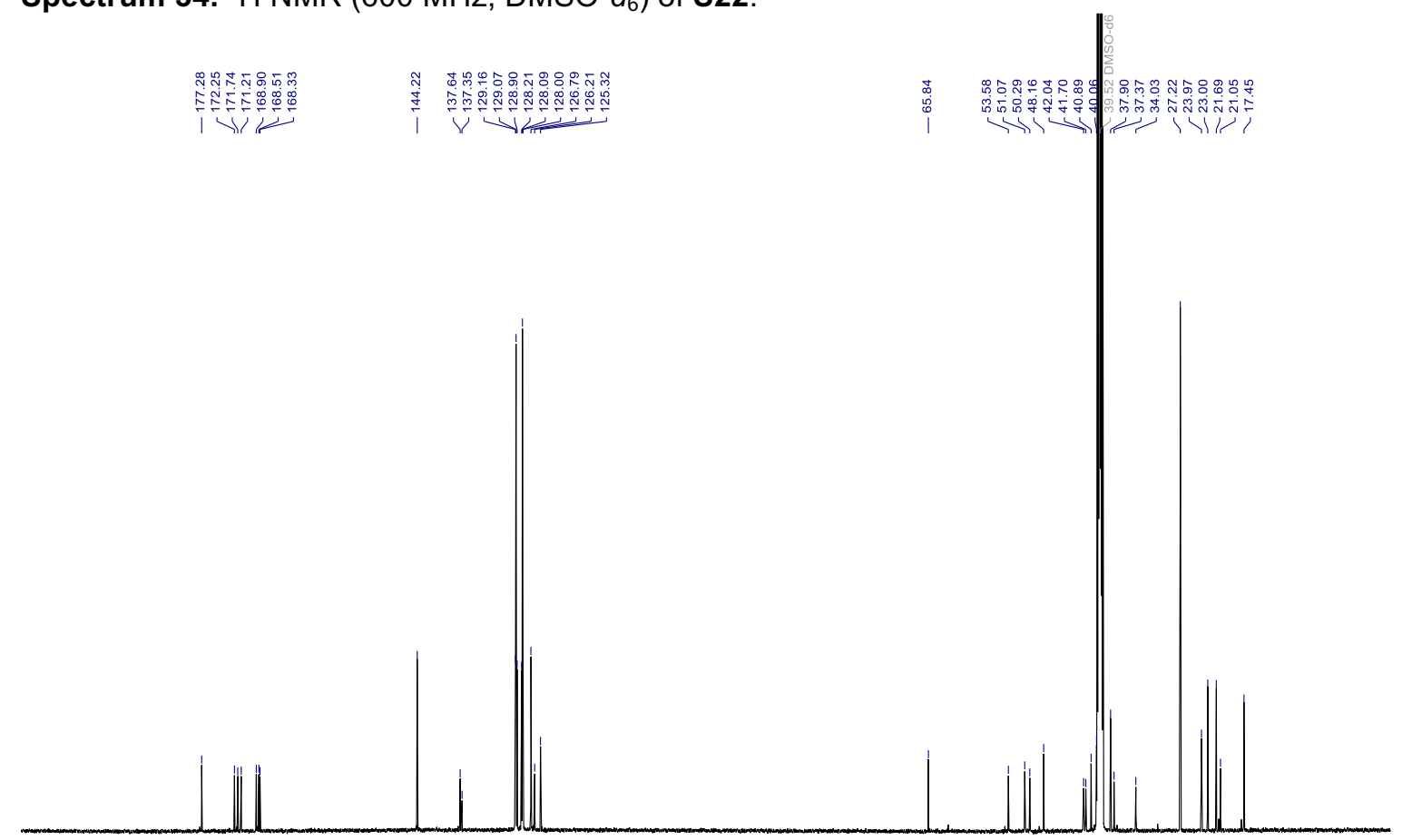

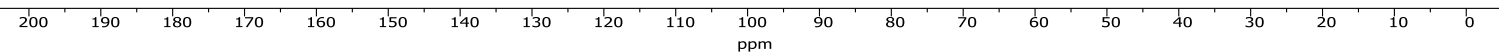

Spectrum 35. ${ }^{13} \mathrm{C}$ NMR $\left(151 \mathrm{MHz}\right.$, DMSO- $\left.d_{6}\right)$ of $\mathbf{S 2 2}$. 
SI Echavarren et al, 'Sequence-Selective Decapeptide Synthesis by the Parallel Operation of ...'
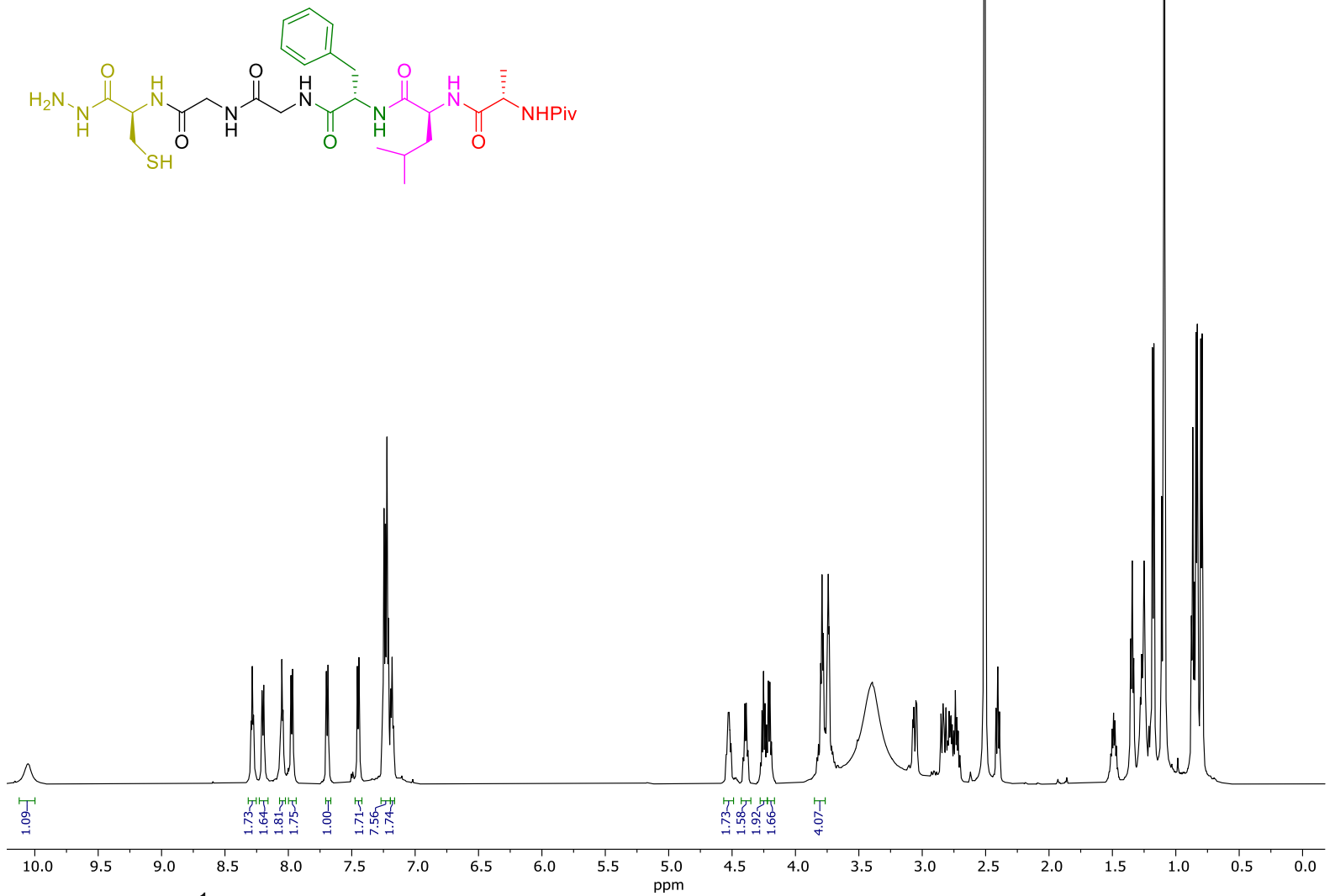

Spectrum 36. ${ }^{1} \mathrm{H}$ NMR $\left(600 \mathrm{MHz}\right.$, DMSO- $\left.d_{6}\right)$ of $\mathbf{1 0}$.

\begin{tabular}{|c|c|}
\hline 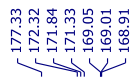 & 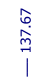 \\
\hline
\end{tabular}
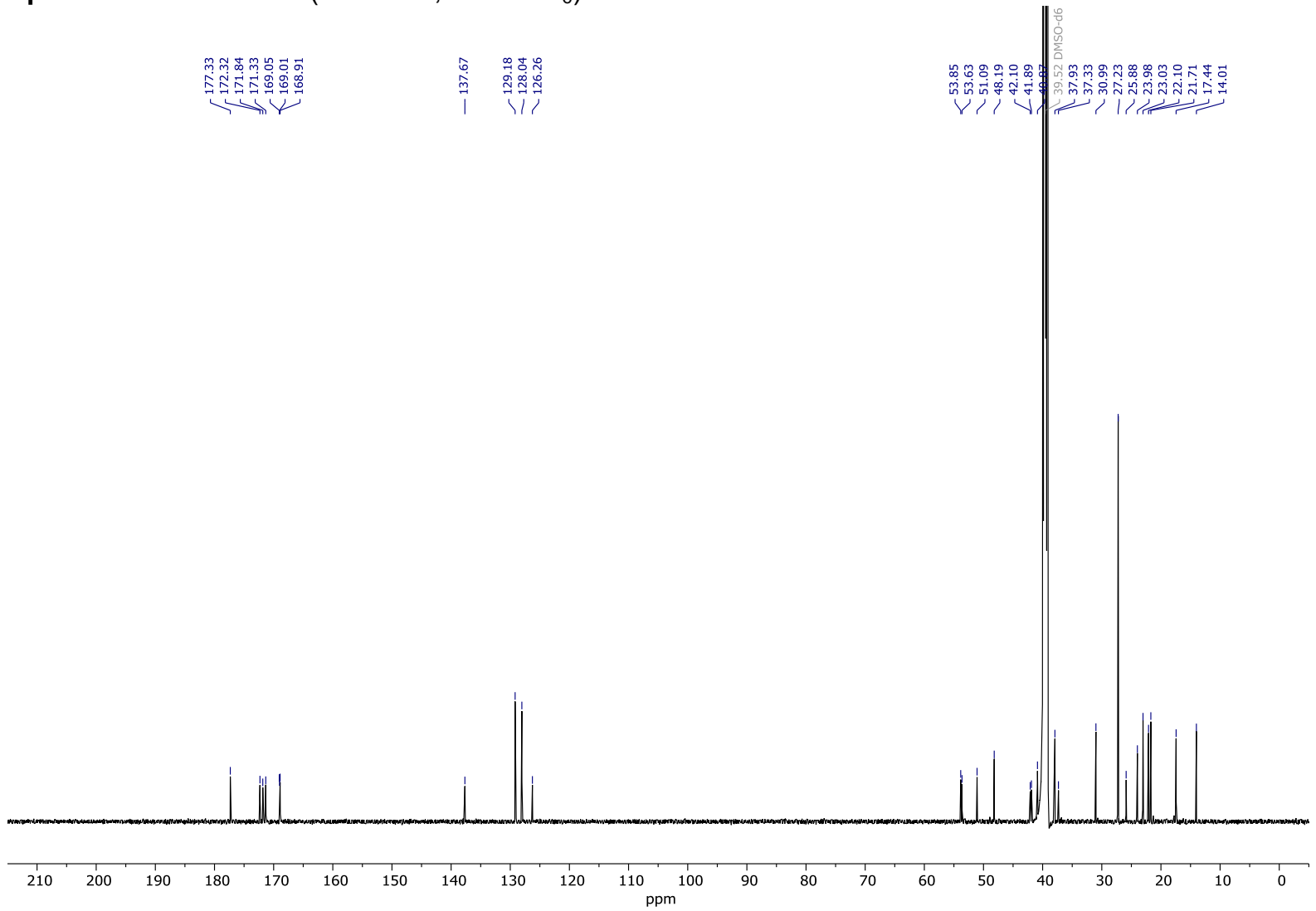

Spectrum 37. ${ }^{1} \mathrm{H}$ NMR $\left(600 \mathrm{MHz}, \mathrm{DMSO}-d_{6}\right)$ of 10. 


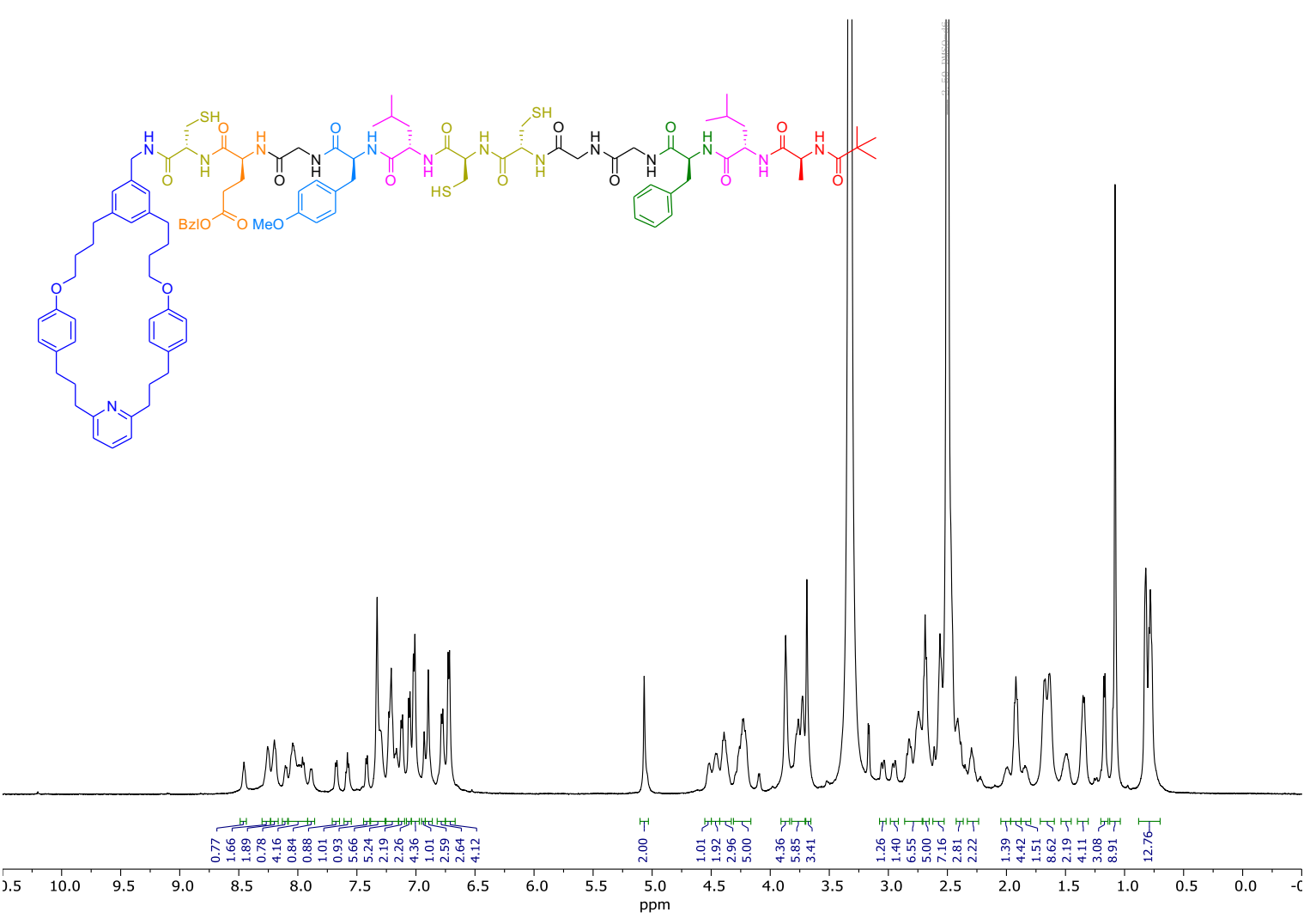

Spectrum 38. ${ }^{1} \mathrm{H}$ NMR $\left(600 \mathrm{MHz}, \mathrm{DMSO}-d_{6}\right)$ of 11.

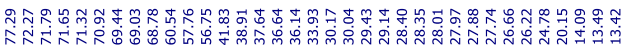

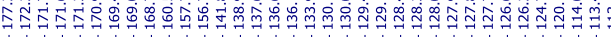
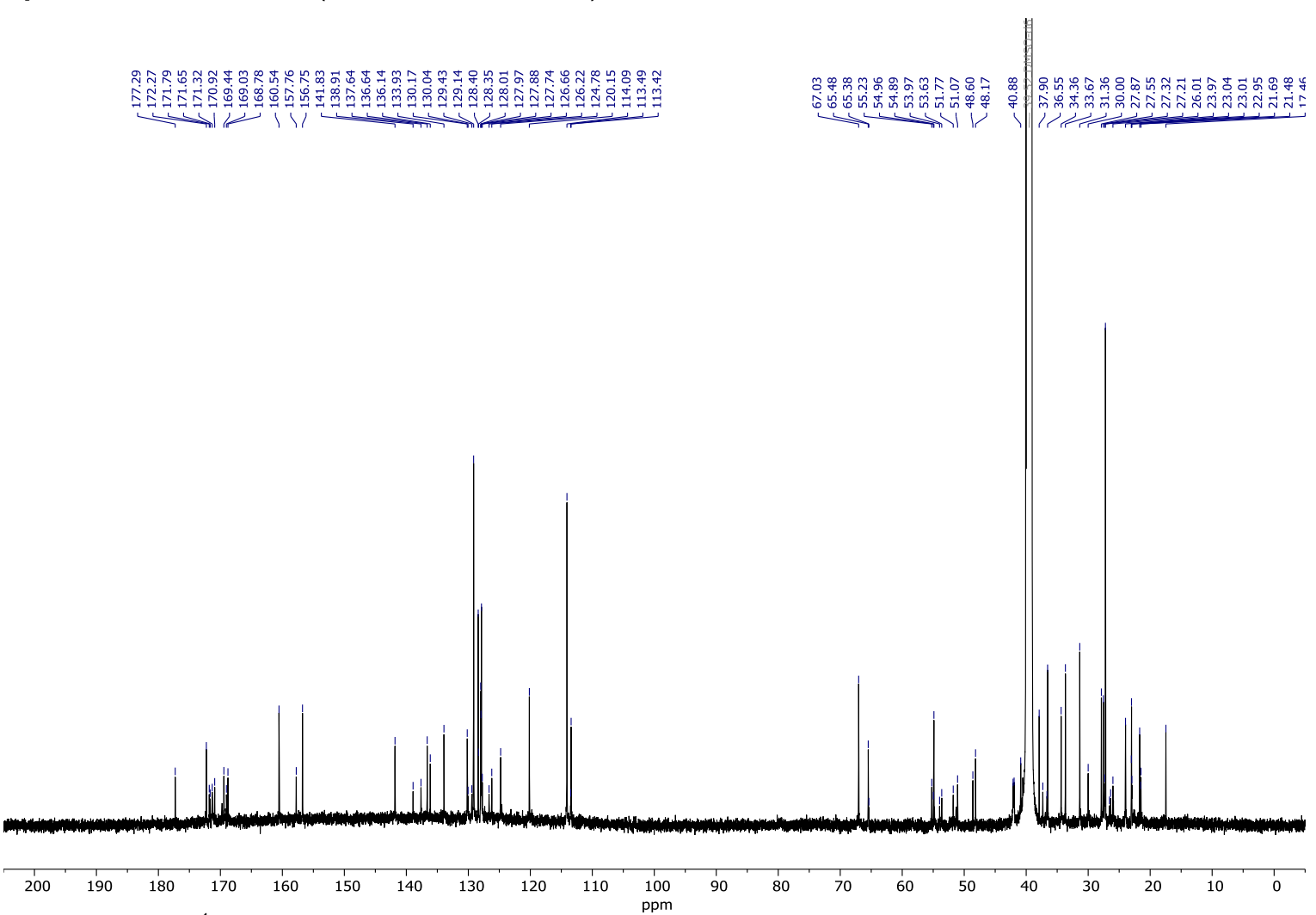

Spectrum 39. ${ }^{1} \mathrm{H}$ NMR $\left(600 \mathrm{MHz}, \mathrm{DMSO}-d_{6}\right)$ of 11 
SI Echavarren et al, 'Sequence-Selective Decapeptide Synthesis by the Parallel Operation of ...'

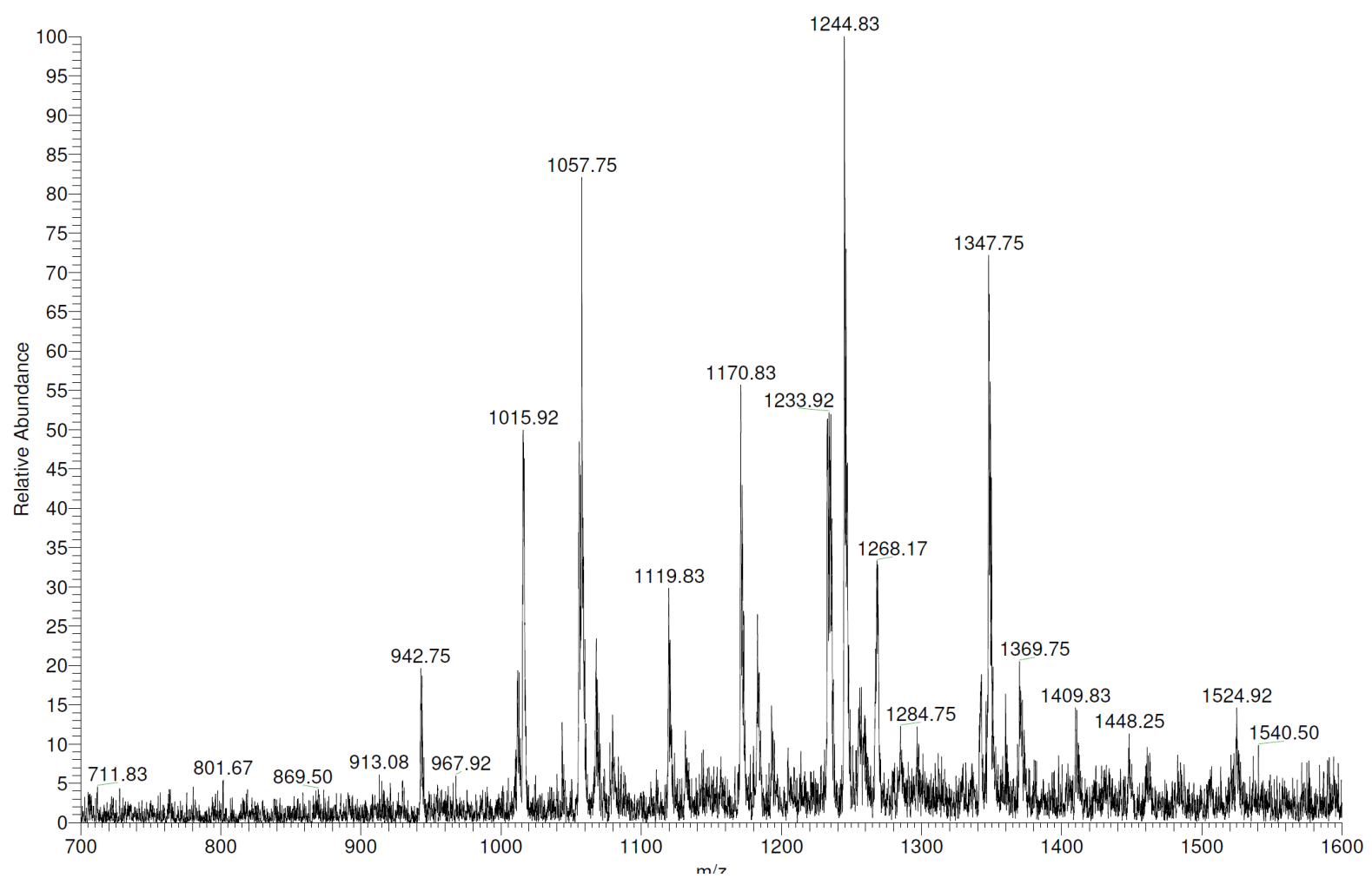

Spectrum 40. Mass spectrum of operation of 1.

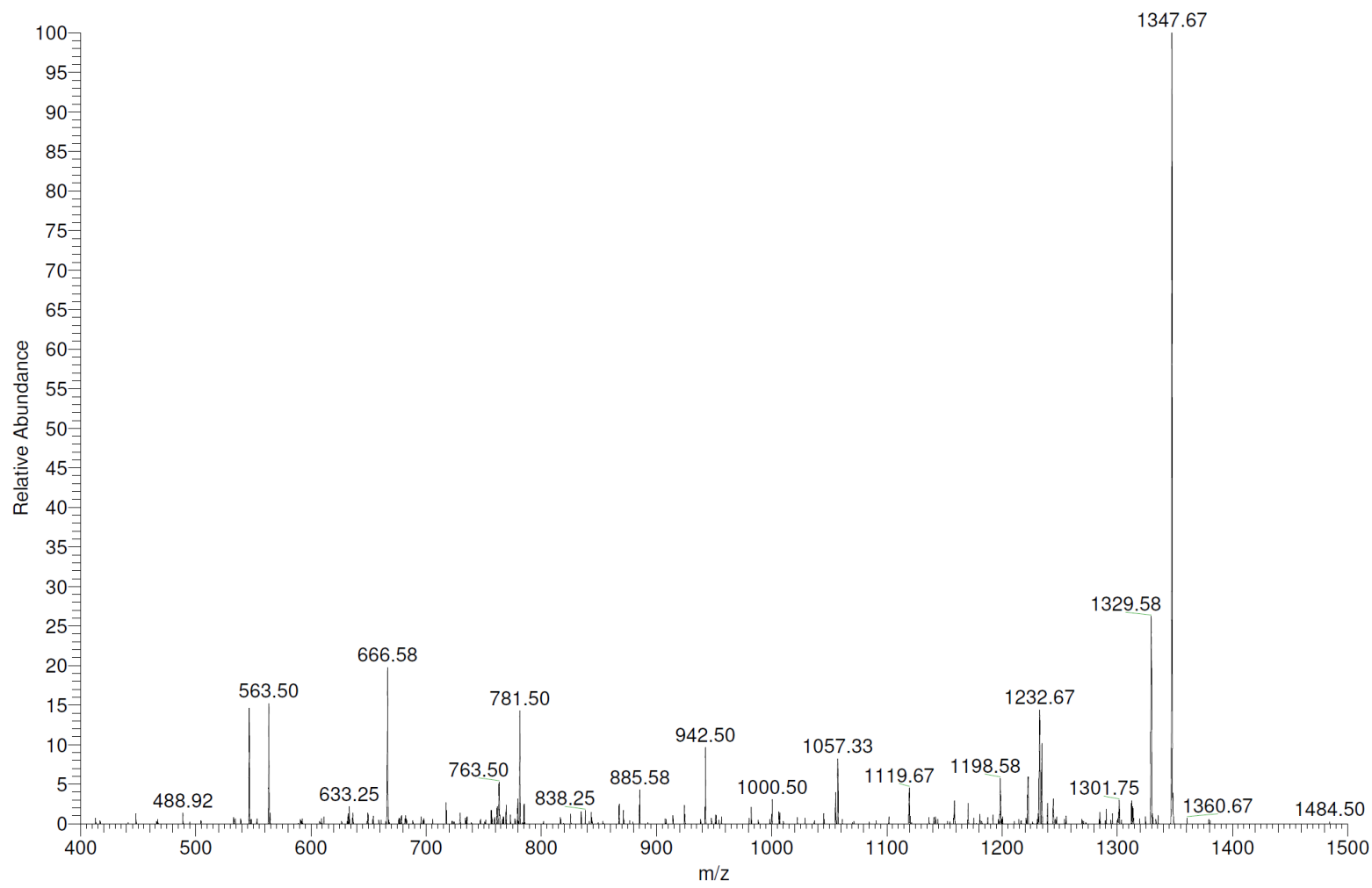

Spectrum 41. MS-MS spectrum of product of operation 7. 
SI Echavarren et al, 'Sequence-Selective Decapeptide Synthesis by the Parallel Operation of ...'

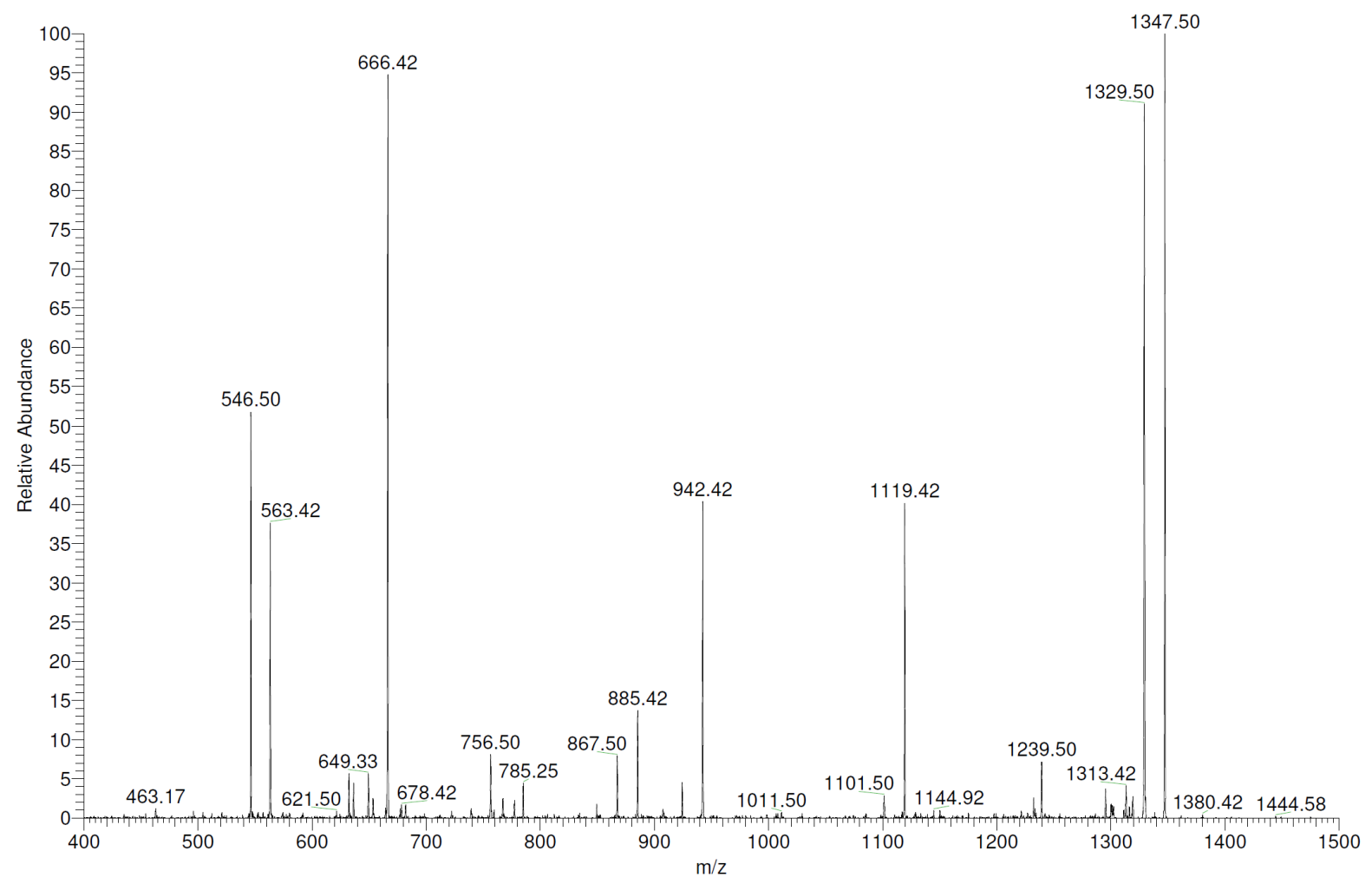

Spectrum 42. MS-MS spectrum of 7 prepared by conventional synthesis.

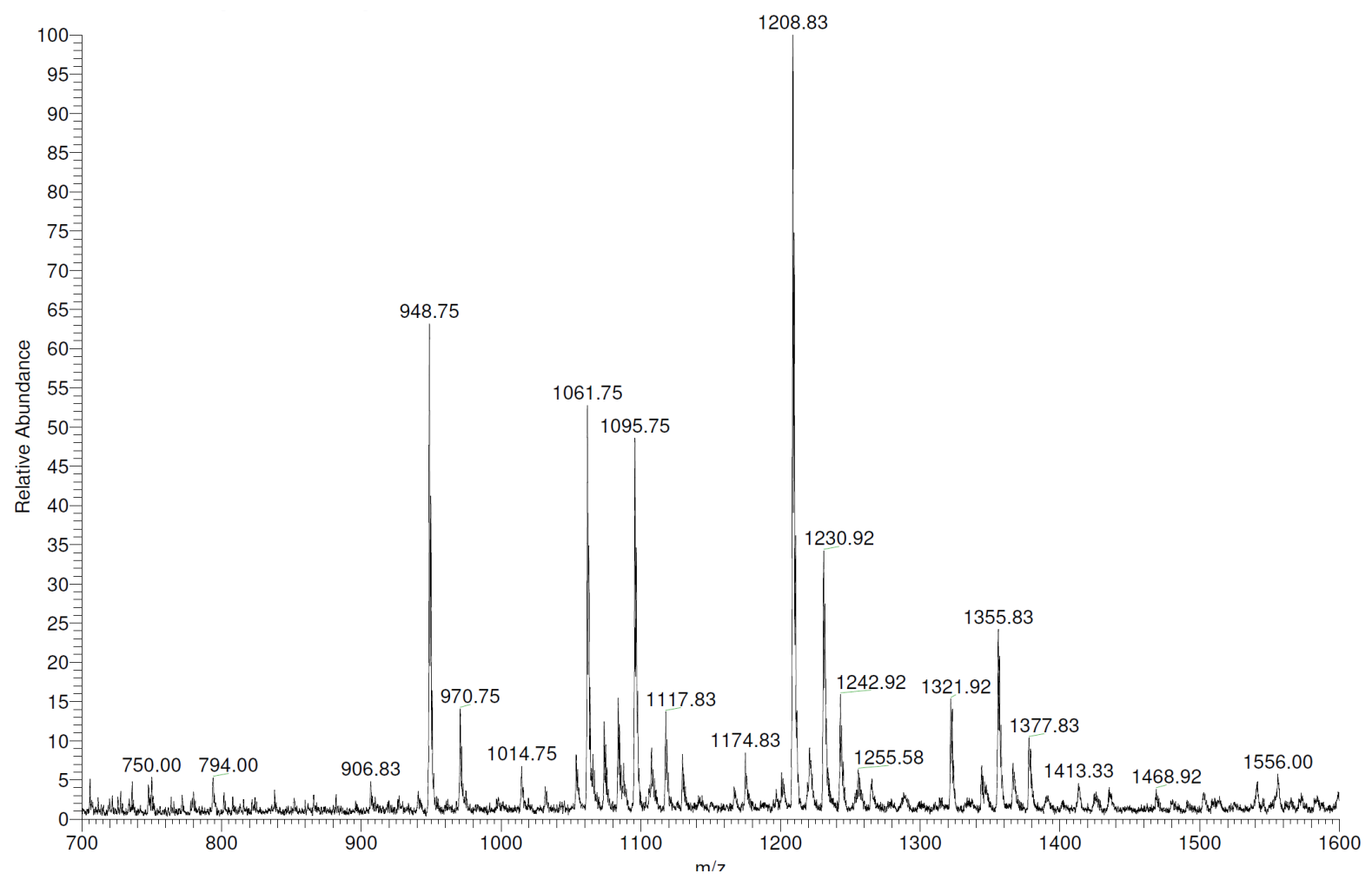

Spectrum 43. Mass spectrum of operation of 2. 
SI Echavarren et al, 'Sequence-Selective Decapeptide Synthesis by the Parallel Operation of ...'

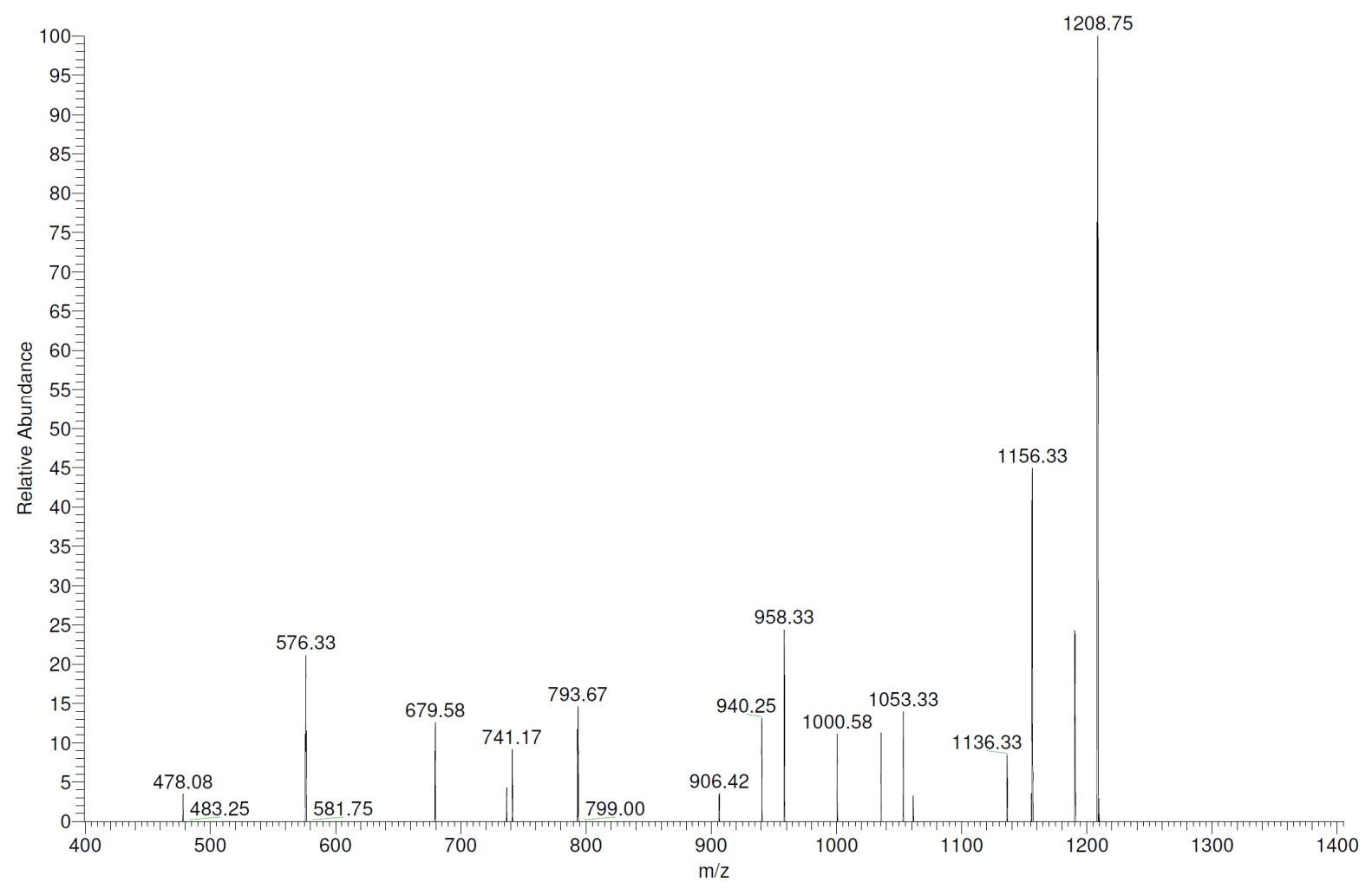

Spectrum 44. MS-MS spectrum of product of operation 8.

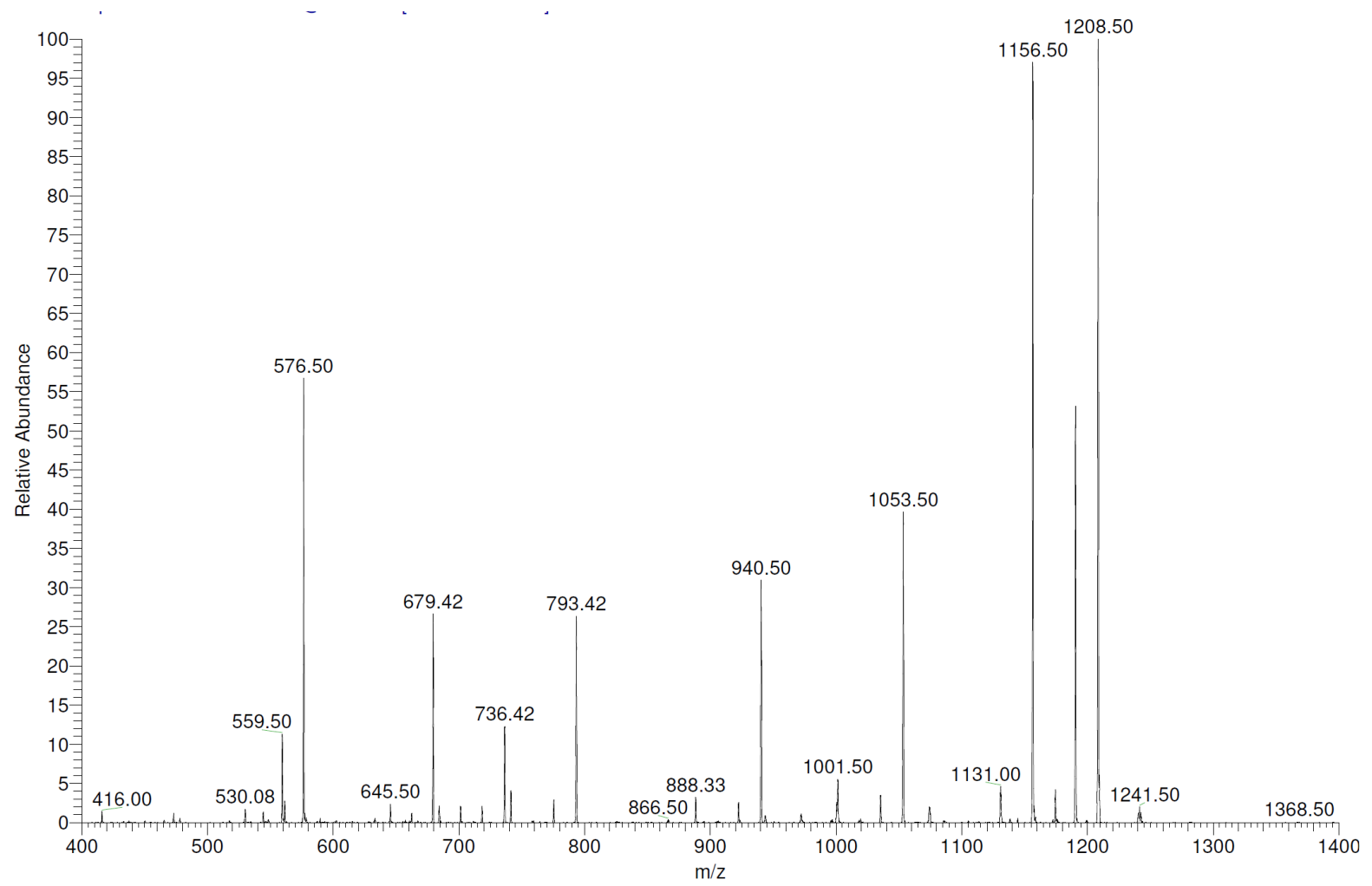

Spectrum 45. MS-MS spectrum of 8 prepared by conventional synthesis. 


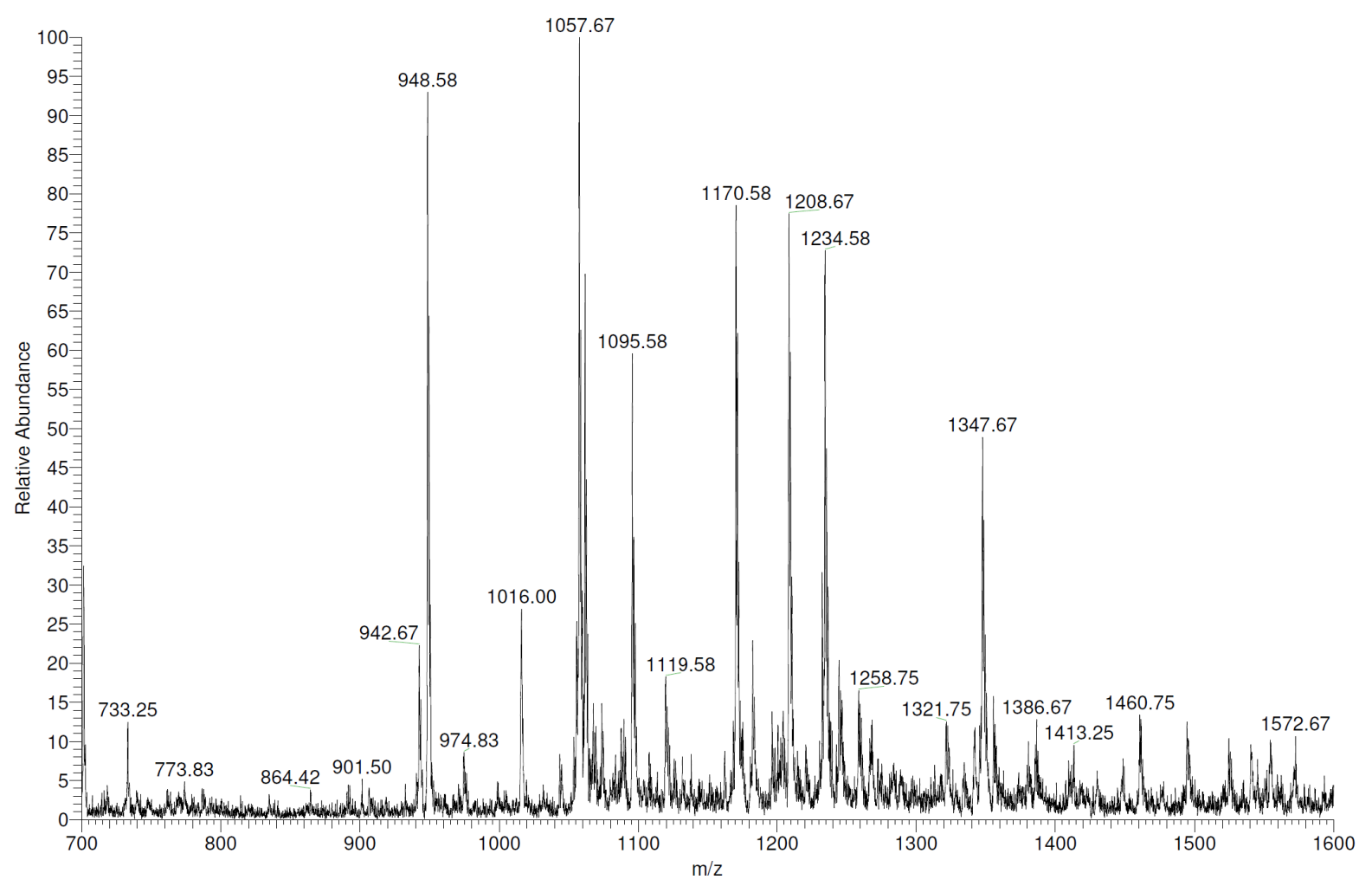

Spectrum 46. Mass spectrum of operation of 1 and 2 parallel in one-pot.

\section{References}

(1) Aucagne, V.; Hanni, K. D.; Leigh, D. A.; Lusby, P. J.; Walker, D. B. Catalytic "Click" Rotaxanes: A Substoichiometric Metal-Template Pathway to Mechanically Interlocked Architectures. J. Am. Chem. Soc. 2006, 128, 2186-2187.

(2) Lewandowski, B.; De Bo, G.; Ward, J. W.; Papmeyer, M.; Kuschel, S.; Aldegunde, M. J.; Gramlich, P. M. E.; Heckmann, D.; Goldup, S. M.; D'Souza, D. M.; Fernandes, A. E.; Leigh, D. A. Sequence-Specific Peptide Synthesis by an Artificial Small-Molecule Machine. Science 2013, 339, 189-193.

(3) De Bo, G.; Kuschel, S.; Leigh, D. A.; Lewandowski, B.; Papmeyer, M.; Ward, J. W. Efficient Assembly of Threaded Molecular Machines for Sequence-Specific Synthesis. J. Am. Chem. Soc. 2014, 136, 5811-5814.

(4) De Bo, G.; Gall, M. A. Y.; Kitching, M. O.; Kuschel, S.; Leigh, D. A.; Tetlow, D. J.; Ward. J. W. Sequence-Specific $\beta$-Peptide Synthesis by a Rotaxane-Based Molecular Machine. J. Am. Chem. Soc. 2017, 139, 10875-10879.

(5) Bo, G.; Gall, M. A. Y.; Kuschel, S.; De Winter, J.; Gerbaux, P.; Leigh, D. A. An artificial molecular machine that builds an asymmetric catalyst. Nat. Nanotechnol. 2018, 13, 381-385. 